Anukul Jana

Unprecedented Synthetic and Reactivity Studies of Compounds with Low Valent Group 14 Elements 



\title{
Unprecedented Synthetic and Reactivity Studies of Compounds with Low Valent Group 14 Elements
}

\author{
Dissertation \\ zur Erlangung des Doktorgrades \\ der Mathematisch-Naturwissenschaftlichen Fakultäten \\ der Georg-August-Universität zu Göttingen
}

vorgelegt von

Anukul Jana

aus Krishnakhayratibar

(Indien)

Göttingen 2009 
D 7

Referent: Prof. Dr. Dr. h. c. mult. H. W. Roesky

Korreferent: Prof. Dr. Dietmar Stalke

Tag der mündlichen Prüfung: 22.10.2009 
Dedicated to my parents for their love and affection 


\section{Acknowledgement}

First of all I want give grateful thanks to Professor Dr. Dr. h. c. mult. Herbert W. Roesky for giving me scientific advice, guidance, motivation, suggestions, and discussions, and most importantly giving the task how to tackle the veritable challenges in synthetic chemistry throughout this work. Very especially for reading and correcting almost everything I "produced", thanks a lot Herbert.

I thank Prof. Dietmar Stalke, Prof. Carola Schulzke, Dr. R. Herbst-Irmer, Dr. G. Schwab, Mrs. I. Objartel, Dr. A. Pal, Mr. A. Döring, Mr. T. Beck, and Mr. P. P. Samuel for their help in the X-ray crystal structural investigations. I thank Prof. Swapan K. Pati and Mr. S. Dutta for their help in theoretical calculations. I thank Mr. W. Zolke, Mr. R. Schöne, and Dr. M. John (NMR spectra), Mr. T. Schuchardt (mass spectra), Mr. M. Hesse (IR spectra), Mr. M. Schlote and the staff of the Analytical Laboratories and Werkstatt for their timely support during this research work. I am also thankful to all the members of glass blowing section, work shop, chemical store as well the security of our institute for their cooperation and help in all kind of situations. I thank all my colleagues and friends in Göttingen for the good and motivating working atmosphere. I am grateful to Dr. G. Nikiforov, Dr. C.-W. So, Dr. Z. Yang, Dr. D. Ghoshal, Dr. S. Nagendran, Dr. S. K. Mandal, Dr. S. Singh, Dr. V. M. JimenezPerez, Dr. N. D. Reddy, Dr. P. M. Gurubasvaraj, Dr. N. Sharanappa, Dr. R. S. Ghadwal, Dr. Y. Ying, Dr. G. Tavcar, Dr. A. Roy, Dr. R. Srivastava, Dr. D. Koley, Dr. J. Li, Dr. S. Demeshko, Dr. A. Singh, Mr. Z. Zhang, Mr. S. P. Sarish, Mr. S. S. Sen, Mr. B. Nekoueishahraki, Mr. S. Ranjit, Mr. A. Das, Mr. V. Nair, Mr. H. Kumar, Mr. C. Abad, and Mr. A. Mandal for their cooperation and help during the course of this work. I will thank to Mrs. C. Roesky for her caring and motherly attitude. I am thankful to Dr. A. C. Stückl for her kind help in the administrative work. I thank Dr. R. Oswald for his timely assistance in computer related work. Also I thank my teachers and friends, who are helping me a lot before starting my research work. I thank my family and friends for their full support and encouragement. The financial support from the Deutsche Forschungsgemeinschaft is gratefully acknowledged. 


\section{Table of Contents}

\section{Introduction}

1.1. $\beta$-Diketiminate ligands

1.2. Halides of Germanium(II) and Tin(II) 3

1.3. Hydrides of Germanium(II) and Tin(II) 5

1.4. Divalent Germanium(II) and Tin(II) Compounds of the Type LMR 5

1.5. Six-Member N-Heterocyclic Silicon(II) and Germanium(II) Compounds 6

1.6. Hydroxides of Tin(II) Compounds 6

1.7. Amides of Germanium(II) and Tin(II) Compounds 7

1.8. Direction of the Thesis Work 8

2. Syntheses and Characterization of the Germanium(II) and Tin(II) Halides 9-14

2.1. Synthesis of Tin(II) Fluoride 9

2.2. Synthesis of Germanium(II) and Tin(II) Bromides 11

2.3. Synthesis of Tin(II) Iodide 12

3. Germanium(II) Hydride an Effective Reagent for Hydrogermylation 15-31

\section{Reactions}

3.1. Germylene Hydride Starting Material 15

3.2. Reaction with Carbon Dioxide 16

3.3. Reactions with Ketones 17

3.4. Reactions with Compounds Containing $\mathrm{C} \equiv \mathrm{C}$ Triple Bonds 19

3.5. Reactions with Compound Containing N=N Double Bond 23

3.6. Reactions with Nitrous Oxide 23

3.7. Reactions with Elemental Sulfur 24

3.8. Reaction with Trimethylsilyl Azide 27

3.9. Reactions with Frustrated Lewis Carbene-Borane Pair 30

4. Hydrostannylation Reaction of LSnH with Unsaturated Molecules 32-46

4.1. Stannylene Hydride Starting Material 32

4.2. Reaction with Carbon Dioxide 33

4.3. Reactions with Carbonyl Compounds 34

4.4. Reactions with Compounds Containing $\mathrm{C} \equiv \mathrm{C}$ Triple Bonds 37

4.5. Reactions with Compounds Containing $\mathrm{C}=\mathrm{N}$ Double Bond 41

4.6. Reaction of LSnH with Fluorinated Carbonyl Compounds: Nucleophilic 41 Addition Reaction vs Hydrogen for Fluorine Exchange in C-F Bonds 
5. Synthesis and Reactivity of Six-Membered N-Heterocyclic Germylene

$47-60$

5.1. Synthesis of six-membered N-Heterocyclic Germylene

5.2. Reaction of L'Ge with Ammonia Borane

5.3. Reaction of NHC with Ammonia Borane 50

5.4. Reaction of L'Ge with Ammonia 50

5.5. Synthesis of $\mathrm{LGeNHNH}_{2}$ 52

5.6. Reactions of L'Ge with O-H Bonded Compounds 53

5.7. Reaction of L'Ge with $\mathrm{Ph}_{2} \mathrm{PCl}$ 56

5.8. Reaction of L'Ge with $\mathrm{B}\left(\mathrm{C}_{6} \mathrm{~F}_{5}\right)_{3}$

6. Reactivity of Six Member N-Heterocyclic Silylene

6.1. Activation of Ammonia

6.2. Insertion of Silylene into N-H bond of Hydrazines

6.3. $[1+4]$ Cycloaddition of Silylene with Diphenyl Hydrazone

7. Diazo Alkane an Effective Reagent for Unprecedented Reactions with Compounds of Low Valent Group 14 Elements

7.1. Reactions of Diazo Compounds with LGeH

7.2. Reaction of Trimethylsilyl Diazomethane with LGeMe

7.3. Reaction of Trimethylsilyl Diazomethane with L'Ge

7.4. Reaction of Lithiated Trimethylsilyl Diazomethane with $\mathrm{LSnCl}$

8. Synthesis and Reactivity of Germanium(II) and Tin(II) Compounds of the type LMR

8.1. Synthesis of $\mathrm{LGeNMe}_{2}$ and its Reaction with Elemental Sulfur

8.2. Synthesis of LGeOTf

8.3. Synthesis of LSnMe

8.4. Synthesis of Tin(II) Amides

8.5. Reaction of $\mathrm{LSnNMe}_{2}$ with Ketones

8.6. Reaction of $\mathrm{LSnNMe}_{2}$ with Terminal Alkines

9. Synthesis of a Monomeric Tin(II) Hydroxide

9.1. Synthesis of $\mathrm{LSnClFe}(\mathrm{CO})_{4}$

9.2. Synthesis of $\mathrm{LSn}\left(\mathrm{NMe}_{2}\right) \mathrm{Fe}(\mathrm{CO})_{4}$

9.3. Synthesis of a Monomeric Tin(II) Hydroxide 
11.1. General Procedures

11.2. Physical Measurements

101

11.3. Starting Materials

101

11.4. Syntheses of Compounds

101

11.4.1. Synthesis of $\operatorname{LSnF}(\mathbf{1})$

101

11.4.2. Synthesis of $\mathrm{LGeBr}(2)$

102

$\begin{array}{ll}\text { 11.4.3. Synthesis of } \operatorname{LSnBr}(3) & 102\end{array}$

11.4.4. Synthesis of LSnI (4) 102

$\begin{array}{lr}\text { 11.4.5. Synthesis of } \mathrm{LGeH}(5) & 102\end{array}$

11.4.6. Synthesis of $\mathrm{LGeOC}(\mathrm{O}) \mathrm{H}(\mathbf{6}) \quad 102$

11.4.7. Synthesis of $\mathrm{LGeOCHPh}\left(\mathrm{CF}_{3}\right)(7) \quad 104$

11.4.8. Synthesis of $\mathrm{LGeOCH}\left(2-\mathrm{C}_{4} \mathrm{H}_{3} \mathrm{~S}\right)\left(\mathrm{CF}_{3}\right)(\mathbf{8}) \quad 104$

11.4.9. Synthesis of $\mathrm{LGeC}\left(\mathrm{CO}_{2} \mathrm{Me}\right) \mathrm{CH}_{2}$ (9) 104

11.4.10. Synthesis of $\mathrm{LGeC}\left(\mathrm{CO}_{2} \mathrm{Et}\right) \mathrm{CH}_{2}(\mathbf{1 0}) \quad 103$

11.4.11. Synthesis of $\mathrm{LGeC}\left(\mathrm{CO}_{2} \mathrm{Me}\right) \mathrm{CHCO}_{2} \mathrm{Me}(\mathbf{1 1}) \quad 105$

11.4.11. Synthesis of $\mathrm{LGeC}\left(\mathrm{CO}_{2} \mathrm{Et}\right) \mathrm{CHCO}_{2} \mathrm{Et}(\mathbf{1 2}) \quad 105$

11.4.13. Synthesis of $\mathrm{LGeC}\left(\mathrm{CO}_{2} t \mathrm{Bu}\right) \mathrm{CHCO}_{2} t \mathrm{Bu}(\mathbf{1 3}) \quad 105$

11.4.14. Synthesis of $\mathrm{LGe}\left(\mathrm{NHCO}_{2} \mathrm{Et}\right) \mathrm{NCO}_{2} \mathrm{Et}(\mathbf{1 4}) \quad 104$

$\begin{array}{ll}\text { 11.4.15. Synthesis of } \mathrm{LGeOH}(\mathbf{1 5}) & 106\end{array}$

11.4.16. Synthesis of LGe(S)SH (16) 106

11.4.17. Synthesis of $\mathrm{LGeN}_{3}(\mathbf{1 7})$ and $\mathrm{L}^{\prime} \mathrm{Ge}\left(\mathrm{NHSiMe}_{3}\right)_{2}(\mathbf{1 8}) \quad 106$

$\begin{array}{ll}\text { 11.4.18. Synthesis of L'Ge (19) } & 105\end{array}$

11.4.19. Synthesis of LSnH (20) 107

11.4.20. Synthesis of $\operatorname{LSnOC}(\mathrm{O}) \mathrm{H}(\mathbf{2 1}) \quad 107$

$\begin{array}{ll}\text { 11.4.21. Synthesis of } \mathrm{LSnOCH}_{2} \mathrm{Fc}(\mathbf{2 2}) & 107\end{array}$

11.4.22. Synthesis of $\mathrm{LSnOCHPh}_{2}(\mathbf{2 3}) \quad 106$

11.4.23. Synthesis of LSnOCHPh(2-Py) (24) 108

$\begin{array}{ll}\text { 11.4.24. Synthesis of } \mathrm{LSnOCH}(2-\mathrm{Py})_{2}(\mathbf{2 5}) & 108\end{array}$

11.4.25. Synthesis of $\mathrm{LSnOCH}\left(\mathrm{C}_{3} \mathrm{H}_{5}\right)_{2}$ (26) 108

11.4.26. Synthesis of $\mathrm{LSnC}\left(\mathrm{CO}_{2} \mathrm{Me}\right) \mathrm{CH}_{2}$ (27) 107

11.4.27. Synthesis of $\mathrm{LSnC}\left(\mathrm{CO}_{2} \mathrm{Et}\right) \mathrm{CH}_{2}$ (28) 109

11.4.28. Synthesis of $\mathrm{LSnC}\left(\mathrm{CO}_{2} \mathrm{Et}\right) \mathrm{CHMe} \mathrm{(29)} 109$

11.4.29. Synthesis of $\mathrm{LSnC}\left(\mathrm{CO}_{2} \mathrm{Me}\right) \mathrm{CHCO}_{2} \mathrm{Me} \mathrm{(30)} 109$ 
11.4.30. Synthesis of $\mathrm{LSnC}\left(\mathrm{CO}_{2} \mathrm{Et}\right) \mathrm{CHCO}_{2} \mathrm{Et}(\mathbf{3 1})$

11.4.31. Synthesis of $\mathrm{LSnC}\left(\mathrm{CO}_{2} t \mathrm{Bu}\right) \mathrm{CHCO}_{2} t \mathrm{Bu}$ (32)

108

11.4.32. Synthesis of 33

11.4.33. Synthesis of 34

11.4.34. Synthesis of $\mathrm{LSnOCHPhCF}_{3}(35)$

110

11.4.35. Synthesis of $\mathrm{LSnOCH}\left(2-\mathrm{C}_{4} \mathrm{H}_{3} \mathrm{~S}\right) \mathrm{CF}_{3}(\mathbf{3 6})$

11.4.36. Synthesis of $\mathrm{LSnOCHPh}\left(\mathrm{C}_{6} \mathrm{~F}_{5}\right)$ (37)

11.4.37. Synthesis of $\mathrm{LSnOCH}\left(\mathrm{C}_{6} \mathrm{~F}_{5}\right)_{2}(\mathbf{3 8})$

11.4.38. Synthesis of $\mathrm{LSnOCHPh}\left(4-\mathrm{C}_{6} \mathrm{H}_{4} \mathrm{~F}\right)(\mathbf{3 9})$

11.4.39. Synthesis of $\mathrm{LSnOCH}_{2} \mathrm{C}_{6} \mathrm{~F}_{5}$ (40)

11.4.40. Synthesis of $(\mathrm{CH} t \mathrm{BuN})_{2} \mathrm{CH}_{2}$ (41)

11.4.41. Synthesis of $\mathrm{LGeNH}_{2}$ (42)

11.4.42. Synthesis of $\mathrm{LGeNHNH}_{2}$ (43)

11.4.43. Synthesis of $\mathrm{LGeOPh}$ (44)

11.4.44. Synthesis of $\mathrm{LGeOC}_{6} \mathrm{~F}_{5}$ (45)

11.4.45. Synthesis of LGeOC(O)Ph (46)

11.4.46. Synthesis of $\mathrm{L}^{\prime} \mathrm{GePPh}_{2}$ (47)

11.4.47. Synthesis of $\mathrm{L}^{\prime \prime} \mathrm{GeB}\left(\mathrm{C}_{6} \mathrm{~F}_{5}\right)_{3}$ (48)

11.4.48. Synthesis of $\mathrm{L}^{\prime \prime \prime} \mathrm{GeB}\left(\mathrm{C}_{6} \mathrm{~F}_{5}\right)_{3}$ (49)

11.4.49. Synthesis of $\mathrm{L}^{\prime} \mathrm{Si}(\mathrm{H}) \mathrm{NH}_{2}$ (51)

11.4.50. Synthesis of $\mathrm{L}^{\prime} \mathrm{Si}(\mathrm{H}) \mathrm{NHNH}_{2}$ (52)

11.4.51. Synthesis of L'Si(H)NHNHMe (53)

11.4.52. Synthesis of 54

11.4.53. Synthesis of $\mathrm{LGeNHNCHCO}_{2} \mathrm{Et}(\mathbf{5 5}) \quad 114$

11.4.54. Synthesis of $\mathrm{LGeNHNCHSiMe}_{3}(\mathbf{5 6}) \quad 113$

11.4.55. Synthesis of $\mathrm{L}^{\prime} \mathrm{Ge}(\mathrm{Me})\left(\mathrm{NHNCHSiMe}_{3}\right)(\mathbf{5 7}) \quad 115$

11.4.56. Synthesis of $\left.\mathrm{LGeC}_{(\mathrm{N}}\right) \mathrm{SiMe}_{3}(\mathbf{5 8}) \quad 115$

$\begin{array}{ll}\text { 11.4.57. Synthesis of } \mathrm{LGeN}(\mathrm{NC}) \mathrm{SiMe}_{3} \text { (59) } & 115\end{array}$

11.4.58. Synthesis of $\mathrm{LSnNCNSiMe}_{3}(\mathbf{6 0}) \quad 114$

$\begin{array}{ll}\text { 11.4.59. Synthesis of } \mathrm{LGeNMe}_{2}(\mathbf{6 1}) & 116\end{array}$

11.4.60. Synthesis of $\mathrm{LGe}(\mathrm{S}) \mathrm{NMe}_{2}(62) \quad 116$

11.4.61. Synthesis of LGeOTf $(\mathbf{6 3}) \quad 116$

$\begin{array}{ll}\text { 11.4.62. Synthesis of LSnMe (64) } & 116\end{array}$ 
11.4.63. Synthesis of $\mathrm{LSnN}\left(\mathrm{SiMe}_{3}\right)_{2}(\mathbf{6 5})$

11.4.64. Synthesis of $\mathrm{LSnNMe}_{2}$ (66)

11.4.65. Synthesis of $\mathrm{LSnOCPh}(2-\mathrm{Py})\left(\mathrm{NMe}_{2}\right)$ (67)

11.4.66. Synthesis of $\mathrm{LSnOCPh}\left(\mathrm{CF}_{3}\right)\left(\mathrm{NMe}_{2}\right)(\mathbf{6 8})$

11.4.67. Synthesis of $\mathrm{LSnCCCO}_{2} \mathrm{Me}(69)$

11.4.68. Synthesis of $\mathrm{LSnCCCO}_{2} \mathrm{Et}$ (70)

11.4.69. Synthesis of $\mathrm{LSnClFe}(\mathrm{CO})_{4}$ (71)

11.4.70. Synthesis of $\mathrm{LSn}\left(\mathrm{NMe}_{2}\right) \mathrm{Fe}(\mathrm{CO})_{4}(\mathbf{7 2})$

11.4.71. Synthesis of $\mathrm{LSn}(\mathrm{OH}) \mathrm{Fe}(\mathrm{CO})_{4}$ (73) 


\section{Abbreviations}

$\delta$

$\lambda$

$\mu$

$\tilde{v}$

av

C

calcd.

$\mathrm{Cp}$

Cp*

EI

eqv.

$\mathrm{eV}$

g

h

$\mathrm{Hz}$

IR

J

K

L

M

$\mathrm{m} / \mathrm{z}$

Mp

$\mathrm{M}^{+}$

$\mathrm{Me}$

Et

$i \operatorname{Pr}$

$t \mathrm{Bu}$

$\mathrm{Ph}$

Ar

$\mathrm{Fc}$

MS chemical shift

wavelength

bridging

wave number

average

Celsius

calculated

cyclopentadienyl

pentamethylcyclopetadienyl

electron impact ionization

equivalents

electron volt

grams

hours

Hertz

infrared

coupling constant

Kelvin

ligand

metal

mass/charge

melting point

molecular ion

methyl

ethyl

iso-propyl

tert-butyl

phenyl

aryl

ferrocenyl

mass spectrometry, mass spectra 
NMR

ppm

q

$\mathrm{R}$

$\mathrm{S}$

d

$\mathrm{t}$

sept

$\mathrm{m}$

b

THF nuclear magnetic resonance

parts per million

quartet

organic substituents

singlet

doublet

triplet

septet

multiplet

broad

tetrahydrofuran 


\section{Chapter 1}

\section{Introduction}

This section of the thesis gives the background and an overview of the area in several sections of the present work.

\section{1. $\beta$-Diketiminate ligands}

The $\beta$-diketiminate class, generally denoted as "nacnac", or [ $\left.\{\operatorname{ArNC}(\mathrm{R})\}_{2} \mathrm{CH}\right]$ " (where $\mathrm{Ar}=$ aryl and $\mathrm{R}=\mathrm{Me}$ or another group), occupies a rightful place alongside a narrow list of popular ancillary supports, given its ability to stabilize or generate unique coordination environments and to support reactive organometallic reagents or catalysts. ${ }^{[1-7]}$ The "nacnac" ligand skeleton is analogous to the "acac" (acetylacetonate) ligand, but the oxygen atoms are exchanged for nitrogen-based moieties such as NR ( $\mathrm{R}=$ alkyl, silyl, Ar) (Scheme 1.1). As a result, the substituent at the nitrogen donor atom can allow for steric protection at the metal center unlike "acac" could offer. When small moieties such as $\mathrm{H}, \mathrm{Me}$, or the $\mathrm{SiMe}_{3}$ on the nitrogen the substance easily forms dimers and allows higher coordination to the metal center, whereas bulky aryl groups on nitrogen usually lead to the isolation of monomeric species with low coordination numbers at the metal.

The first documented cases of $\beta$-diketiminate metal complexes were reported by McGeachin, ${ }^{[8]}$ Parks, and Holm ${ }^{[9]}$ in 1968 . The explosion in popularity of "nacnac" amongst synthetic chemists is driven, in part, by the monoanionic nature of the $\beta$-diketiminate group, the chelating nature but also variable mode of hapticity, the ease in preparation, and the versatility to tune both electronic and steric parameters. Till to date the $\mathrm{N}$-aryl substituted "nacnac" ligands $[\mathrm{HN}(\mathrm{Ar}) \mathrm{C}(\mathrm{Me}) \mathrm{CHC}(\mathrm{Me}) \mathrm{N}(\mathrm{Ar})]^{[10]}$ and $[\mathrm{HN}(\mathrm{Ar}) \mathrm{C}(t \mathrm{Bu}) \mathrm{CHC}(t \mathrm{Bu}) \mathrm{N}(\mathrm{Ar})]^{[11]}$ $\left(\mathrm{Ar}=2,6-i \mathrm{Pr}_{2} \mathrm{C}_{6} \mathrm{H}_{3}\right)$ showed to be the best for stabilization of low coordinate metal sites.

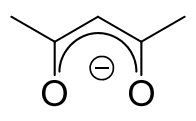

(a)

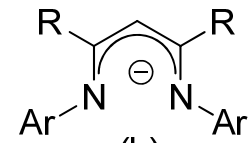

(b)

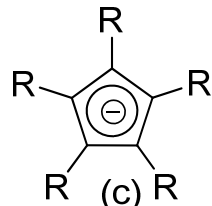

(c) $R$

Scheme 1.1. Schematic diagram of (a) "acac", (b) "nacnac", and (c) "cyclopentadienyl" ligands 
The major breakthrough in this area was achieved in the mid 1990's, when $\beta$-diketiminate ligands were used as spectator ligands, thus offering strong metal-ligand bonds like cyclopentadienyls (Scheme 1.1). In contrast to the latter, $\beta$-diketiminate ligands offer a possibility of subtle tuning of their electronic and steric properties by simple variation of the substituents on nitrogen and adjacent carbon atoms.

The chemistry of the $\beta$-diketiminate ligand since its initial recognition as an ancillary ligand and the effective stabilization of lower oxidation states and lower coordination numbers has experienced a burgeoning due to the steric and electronic properties and a variety of coordination modes. The ligand can be regarded as a bidentate and monoanionic ligand and can be prepared by direct condensation of 2,4-pentanedion, 2,6-diisopropylaniline, and $\mathrm{HCl}$ in boiling ethanol and subsequent neutralization of the generated ligand hydrochloride with $\mathrm{Na}_{2} \mathrm{CO}_{3}$ to obtain free LH (Scheme 1.2). ${ }^{[12]}$<smiles>CC(=O)CC(C)=O</smiles>

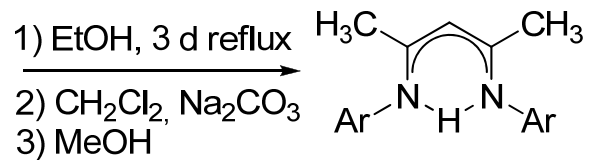

3) $\mathrm{MeOH}$

Scheme 1.2. Preparation of ligand LH

Recently, a great deal of unique and fascinating results reflect the $\beta$-diketiminate ligand stabilizing properties with main group elements. Thus the isolation and structural characterization of a monomeric aluminum(I) compound $\mathrm{LAl}^{[13]}$ and magnesium(I) compound $\mathrm{LMgMgL}$ with $\mathrm{Mg}-\mathrm{Mg}$ bonds ${ }^{[14]}$ were reported.

In this thesis we are describing the utility of bidentate and monoanionic properties of $\beta$ diketiminate ligand with group 14 elements. By using this ligand we are for the first time reporting here the synthesis and reactivity of the very pure monomeric germanium(II) and tin(II)-hydride. Moreover herein we also report the synthesis and reactivity of novel heteroleptic germanium(II) and tin(II) compounds including terminal tin(II) hydroxide.

It has been well established that the $\beta$ - diketiminate ancillary framework has vulnerable sites, especially in the context of early transition metal or main group organometallic chemistry. Of 
the many different modes of transformation involving the $\beta$-diketiminate ligand, perhaps the most common pathway is intramolecular $\mathrm{C}-\mathrm{H}$ bond activation of peripheral groups stemming from the R-nitrogen atoms. ${ }^{[15-19]}$ Other reactions of this ligand type include electrophilic activation at the $\gamma$-carbon due to delocalization of charge about the NCCCN ring, ${ }^{[20-27]}$ as well as deprotonation of the $\beta$-methyl group composing the NCCCN ring. ${ }^{[28-33]}$ The ability of the $\beta$-diketiminate's $\beta$-methyl hydrogens to tautomerize in $\left[\{\operatorname{ArNC}(\mathrm{Me})\}_{2} \mathrm{CH}\right]^{-}(\mathrm{Ar}=2,6-$ $\left.i \mathrm{Pr}_{2} \mathrm{C}_{6} \mathrm{H}_{3}\right)$ allows for facile deprotonation to form the $\mathrm{N}, \mathrm{N}^{\prime}$-chelating, bis-anilide ligand $\left[\mathrm{ArNC}(\mathrm{Me}) \mathrm{CHC}\left(\mathrm{CH}_{2}\right) \mathrm{NAr}\right]^{2-}(\mathrm{Scheme} 1.3)$. Lewis acid coordination to the methylene moiety of $\left[\mathrm{ArNC}(\mathrm{Me}) \mathrm{CHC}\left(\mathrm{CH}_{2}\right) \mathrm{NAr}\right]^{2-}$ can yield charge-separated species. ${ }^{[34]}$ Even though the $\beta$ diketiminate ligand can readily undergo deprotonation of the $\beta$-methyl group, such a transformation can be avoided by incorporating a $t \mathrm{Bu}$ group. ${ }^{[35]}$

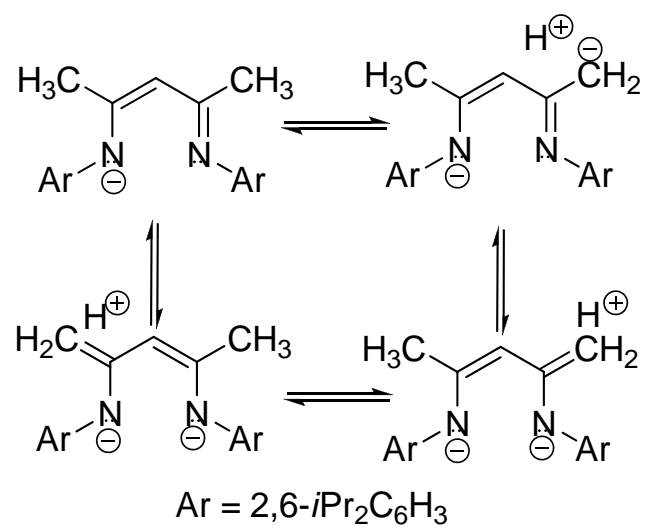

Scheme 1.3. Schematic diagram of bis-anilide ligand $\left[\mathrm{ArNC}(\mathrm{Me}) \mathrm{CHC}\left(\mathrm{CH}_{2}\right)-\mathrm{NAr}\right]^{2-}$

The utility of bidentate and dianionic properties of bis-anilide ligand ([ArNC(Me)CHC( $\left.\mathrm{CH}_{2}\right)$ $\left.\mathrm{NAr}]^{2-}=\mathrm{L}^{\prime} ; \mathrm{Ar}=2,6-i \mathrm{Pr}_{2} \mathrm{C}_{6} \mathrm{H}_{3}\right)$ offers the formation of heterocyclic compounds. Driess et al. reported the synthesis and reactivity of six-membered $\mathrm{N}$-heterocyclic silylene, $\mathrm{L}^{\prime} \mathrm{Si}^{[36]}$ and germylene $\mathrm{L}^{\prime} \mathrm{Ge} .{ }^{[37]}$ In this thesis we independently describe the utility of bidentate and dianionic properties of bis-anilide ligand $\left[\mathrm{ArNC}(\mathrm{Me}) \mathrm{CHC}\left(\mathrm{CH}_{2}\right) \mathrm{NAr}\right]^{2-}$ for the synthesis of six-membered N-heterocyclic germylene and silylene systems.

\subsection{Halides of Germanium(II) and Tin(II)}

Halides of group 14 elements are important precursors for a variety of new reactions. Dihalogermylenes, $\mathrm{GeX}_{2}(\mathrm{X}=\mathrm{F}, \mathrm{Cl}, \mathrm{Br}, \mathrm{I})$ were the first known divalent species that had 
been studied and reviewed as starting materials and intermediates in organogermanium chemistry in $1973 .{ }^{[38]} \mathrm{Cp} * \mathrm{GeCl}$ was the first example of a monohalide with the general composition RMX $\left(M=\right.$ Ge, Sn; $\mathrm{R}=$ organic group; $\mathrm{X}=$ halide) prepared in $1987,{ }^{[39]}$ followed by the tin analogue $\mathrm{RSnCl}\left(\mathrm{R}=\mathrm{C}\left(\mathrm{SiMe}_{3}\right)_{2} \mathrm{C}_{5} \mathrm{H}_{4} \mathrm{~N}-2\right)$ in $1988 .{ }^{[40]}$ After that in 2006 our group reported the synthesis and structure of the first monomeric chloro silylene $\left[\left\{\mathrm{PhC}(\mathrm{N} t \mathrm{Bu})_{2}\right\} \mathrm{SiCl}\right] .{ }^{[41]}$ In this year our group reported on the synthesis of the first stable "bottleable" Lewis base (NHC) stabilised dichloro silylene, ${ }^{[42]}$ which has the potential for further reactions and may have the same impact like $\mathrm{GeCl}_{2} \cdot \mathrm{di}$-oxane. Filippou et al. published the N-heterocyclic carbene adduct of dibromosilylene. ${ }^{[43]}$ Moreover there were a variety of monohalide compounds prepared and their chemistry showed that it is an interesting class of compounds. ${ }^{[10,44]}$ The fluoro compounds are of interest and expected to have a different reactivity due to the strong electron withdrawing effect of fluorine compared to the other halides. Moreover, organometallic fluorides of group 14 elements are important because of their industrial application, synthetic methodology, and theoretical implications. ${ }^{[45-50]}$ In the literature only two dimeric $\mathrm{Sn}(\mathrm{II})$ fluorides were reported as stable molecules, ${ }^{[51]}$ and one Ge(II) fluoride, $\mathrm{PhGeF}$, was studied as a reactive intermediate, ${ }^{[52]}$ also the preparation of $\mathrm{Ge}(\mathrm{II})$ fluoride using the $\beta$-diketiminate ligand was reported by our group. ${ }^{[28]}$ The parent low valent tin(II)difluoride $\left(\mathrm{SnF}_{2}\right)$ is not soluble in any organic solvents and only poorly soluble in water. $\mathrm{SnF}_{2}$ is widely used in toothpaste, as a source of fluoride to harden dental enamel. ${ }^{[53]}$ In the enamel it converts apatite into fluoroapatite, which is more resistant to attack by acids generated by bacteria. In the solid state $\mathrm{SnF}_{2}$ exists as a tetramer containing a puckered eight-membered ring in which Sn and $\mathrm{F}$ atoms are alternately arranged. Four additional $\mathrm{F}$ atoms which are bound to the four tin atoms, provide weaker interactions with $\mathrm{Sn}$ atoms of the adjacent rings. ${ }^{[54]}$ The most characteristic feature of the $\mathrm{SnF}_{2}$ compound is the tendency to interact via Sn-F-Sn bridges and as a result of this, it is only poorly or not soluble in common organic solvents, even at higher temperatures in the gas phase it forms preferentially ring compounds. These difficulties are overcome by the use of a bulky and intramolecularly coordinating ligand. By applying this technique some monomeric organotin(IV)fluoride compounds were recently reported. ${ }^{[55-58]}$ This development of Sn(IV) fluorides encouraged us to synthesize $\mathrm{Sn}$ (II) fluorides. 


\subsection{Hydrides of Germanium(II) and Tin(II)}

Metal hydrides and their complexes are considered valuable synthons in chemistry. It was demonstrated that main-group and transition-metal hydrides are important intermediates in some industrial processes and also function as catalysts. ${ }^{[59-67]}$ Furthermore, in the quest for alternative energy sources, metal hydrides have been considered as potential feedstocks for hydrogen storage. ${ }^{[68-71]}$ In recent years the chemistry of metal hydrides has drawn great attention. ${ }^{[72,73]}$ The hydrides of group 14 elements are important and their chemistry and applications are very rich. ${ }^{[74-78]}$ Group 14 hydrides such as $\mathrm{R}_{3} \mathrm{SiH}, \mathrm{R}_{3} \mathrm{GeH}$, and $\mathrm{R}_{3} \mathrm{SnH}$ are important reagents for some key reactions in organic synthesis. However, almost all such compounds reported to date involve the central element in the +4 oxidation state. In recent years our group ${ }^{[28,79]}$ and Power et al. ${ }^{[80-82]}$ first reported only on the synthesis of germanium(II) and tin(II) hydrides of a divalent group 14 element, although the existence of $\mathrm{MH}_{2}(\mathrm{M}=\mathrm{Si}$ to $\mathrm{Pb})$ has been anticipated by theoretical calculations. ${ }^{[83]}$ Also the parent $\mathrm{SnH}_{2}$ has been prepared and characterised in an argon matrix. ${ }^{[84]}$ At elevated temperature $\mathrm{SnH}_{2}$ changed to an insoluble solid of unknown structure.

Until now no reaction has been reported based on these hydrides. This is mainly due to the synthetic difficulties as well as stability for further reactions. Most of these hydrides of group 14 elements based on +4 oxidation state are performing the reaction in the presence of a metal catalyst or in the presence of a radical initiator such as azobis(isobutyronitrile) (AIBN). Comparison of hydrides of group 14 elements based on +4 oxidation state with those of the +2 oxidation state resulted in more reactive species, because the ionic radii of species with +2 oxidation state are larger when compared with those of the +4 oxidation state. ${ }^{[85]}$ Herein we explore the reactivity studies of hydrides of group 14 elements based on +2 oxidation state towards unsaturated molecules.

\subsection{Divalent Germanium(II) and Tin(II) Compounds of the Type LMR}

Within the resurgence of interest in stable N-heterocyclic carbenes over the years, ${ }^{[86]}$ the chemistry of their heavier analogues, silylenes, germylenes, stannylenes, and plumbylenes, has also attracted considerable interest. Studies showed that compounds of divalent germanium bonded to small alkyl substituents (such as $\mathrm{Me}, \mathrm{Et}, \mathrm{Bu}$ ) are highly reactive, and therefore exist only as intermediates. ${ }^{[87,88]}$ However, the divalent germanium compounds can be stabilized with bulky ligands. Jutzi et al. reported the first example of such compounds by 
oxidative addition of $\mathrm{MeI}$, but failed to characterize these compounds by structural analysis. ${ }^{[89]}$ Therefore it is important to explore the properties of alkylated germanium(II) compounds containing bulky ligands. Recently our group reported the synthesis and structure of $\mathrm{LGeMe}\left[\mathrm{L}=\mathrm{HC}(\mathrm{CMeNAr})_{2}, \mathrm{Ar}=2,6-i \mathrm{Pr}_{2} \mathrm{C}_{6} \mathrm{H}_{3}\right] .{ }^{[29]}$ There has been considerable interest over the past three decades in the chemistry of dialkyl and diaryl Sn(II) compounds. ${ }^{[90-94]}$ But remarkably little is known about the chemistry of simpler heteroleptic derivatives such as $\mathrm{L}^{\prime} \mathrm{SnR}\left(\mathrm{L}^{\prime}=\right.$ chelating ligand or bulky aryl ligand, and $\mathrm{R}$ a small substituent). It is of interest to further develop the chemistry of such compounds. Power et al. reported on the reaction of 2,6-Trip ${ }_{2} \mathrm{C}_{6} \mathrm{H}_{3} \mathrm{Sn}(\mathrm{II}) \mathrm{Cl}$ (Trip $=$ 2,4,6-i $\mathrm{Pr}_{3} \mathrm{C}_{6} \mathrm{H}_{2}$ ) with MeLi. They obtained 2,6Trip $_{2} \mathrm{C}_{6} \mathrm{H}_{3} \mathrm{Sn}(\mathrm{II})-\mathrm{Sn}(\mathrm{IV})(\mathrm{Me})_{2}-2,6-\mathrm{Trip}_{2} \mathrm{C}_{6} \mathrm{H}_{3}$ instead of 2,6-Trip ${ }_{2} \mathrm{C}_{6} \mathrm{H}_{3} \mathrm{Sn}(\mathrm{II}) \mathrm{Me}{ }^{\left[{ }^{95]}\right.}$ Moreover it is mentioned that the dimethylstannylene $\left(\mathrm{SnMe}_{2}\right)$ is a transient species at room temperature, which is generated in situ under special experimental conditions from a variety of substrates and some aspects of its chemistry in solution and gas phase have been reported. ${ }^{[96-98]}$

\subsection{Six-Membered N-Heterocyclic Silicon(II) and Germanium(II) Compounds}

Silylenes are of considerable current interest owing to their fundamental interest and potential applications in transition metal catalysis. ${ }^{[99]}$ Since the first stable N-heterocyclic silylene was reported by West et al. in 1994, ${ }^{[100]}$ several other cyclic silylenes have been synthesized and fully characterized by Lappert, ${ }^{[101]}$ Kira, ${ }^{[102]}$ and others. ${ }^{[103,104]}$ Since the pioneering work on the synthesis of isolable germylenes $\left(\mathrm{R}_{2} \mathrm{Ge}\right)$ reported by Lappert et al. ${ }^{[105]}$ remarkable progress has been made in tuning the reactivity of the low coordinate Ge atom in germylenes

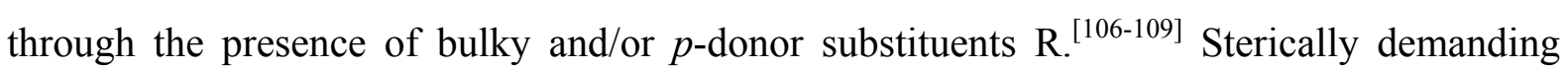
amido or alkoxo substituents at the two coordinate Ge atom lead to derivatives which can serve as ligands with different donor-acceptor abilities for coordination compounds. ${ }^{[110-114]}$ Herein we explored the reactivity studies of six-membered N-heterocyclic silicon(II) and germanium(II) compounds.

\subsection{Hydroxides of Tin(II) Compounds}

The compounds containing the hydroxy group are considered as potential building block in synthetic chemistry. In recent years we were interested in the synthesis of organometallic hydroxides. We reported on the preparation and structural characterization of unusual 
compounds such as $\left[\left(\mathrm{Me}_{3} \mathrm{Si}\right)_{3} \mathrm{CSnO}(\mathrm{OH})\right]_{3},{ }^{[115]} \quad\left[(\mathrm{CpZr})_{6}\left(\mu_{4}-\mathrm{O}\right)(\mu-\mathrm{O})_{4}(\mu-\mathrm{OH})_{8}\right],{ }^{[116]}$ $\left[\mathrm{Cp}_{2} * \mathrm{MMe}(\mathrm{OH})\right](\mathrm{M}=\mathrm{Zr} \text { and } \mathrm{Hf})^{\left[117,{ }^{118]}\right.}\left[\mathrm{LAl}(\mathrm{OH})_{2}\right],{ }^{[119]}[\mathrm{LAlR}(\mathrm{OH})](\mathrm{R}=\mathrm{Me}, \mathrm{Et}$, and $\mathrm{Ph}),{ }^{[120-122]}\left[\mathrm{LGa}(\mathrm{OH})_{2}\right],{ }^{[123]}[\mathrm{LGaMe}(\mathrm{OH})],{ }^{[124]}[\mathrm{LMOH}],(\mathrm{M}=\mathrm{Ca} \text { and } \mathrm{Sr})^{[125,126]}$ and $[\mathrm{LGeOH}]^{[127]}$ where $\mathrm{L}=\mathrm{HC}(\mathrm{CMeNAr})_{2} ; \mathrm{Ar}=2,6-i \mathrm{Pr}_{2} \mathrm{C}_{6} \mathrm{H}_{3}$, and their reactions generating heterobimetallic systems. ${ }^{[5,128-130]}$ Interestingly, only a few examples of well-characterized tin(IV) hydroxides are known in literature, ${ }^{[131]}$ and one dimeric compound based on tin(II) has been reported. ${ }^{[132]}$ This hydroxide was prepared from $\mathrm{Ar}^{\prime} \mathrm{SnSnAr}^{\prime}\left[\mathrm{Ar}^{\prime}=2,6\{(2,6-\right.$ $\left.\left.\left.i \mathrm{Pr}_{2} \mathrm{C}_{6} \mathrm{H}_{3}\right)_{2}\right\} \mathrm{C}_{6} \mathrm{H}_{3}\right]$ and 2,2,6,6-tetramethylpiperidine-N-oxide (TEMPO) or nitrous oxide $\left(\mathrm{N}_{2} \mathrm{O}\right)$ by serendipity. To the best of our knowledge there is presently no report available on a well-designed synthesis and structural characterization of any monomeric tin(II) hydroxide.

\subsection{Amides of Germanium(II) and Tin(II) Compounds}

Stable low-valent amide derivatives of heavier group 14 elements have been known for almost 3 decades. They have the general formula $\mathrm{M}\left(\mathrm{NR}_{2}\right)_{2},(\mathrm{M}=\mathrm{Ge}, \mathrm{Sn}$, and $\mathrm{Pb})$ where the nitrogen substituents can be varied by bulky silyl, aryl, or alkyl groups. ${ }^{[133-136]}$ In 2005 Power et al. reported the use of very crowding terphenyl ligands that enable the synthesis and stabilization of $\mathrm{Ge}(\mathrm{II}), \mathrm{Sn}(\mathrm{II})$, and $\mathrm{Pb}(\mathrm{II})$ amides of formula $\left(\mathrm{Ar} * \mathrm{MNH}_{2}\right)_{2}(\mathrm{M}=\mathrm{Ge}, \mathrm{Sn}$, or $\mathrm{Pb})$ or $\left(\mathrm{Ar}^{\prime} \mathrm{GeNH}_{2}\right)_{2}$, where $\mathrm{Ar} *=2,6\left\{\left(2,4,6-i \mathrm{Pr}_{3} \mathrm{C}_{6} \mathrm{H}_{2}\right)_{2}\right\} \mathrm{C}_{6} \mathrm{H}_{3}$ and $\mathrm{Ar}^{\prime}=2,6\{(2,6-$ $\left.\left.i \mathrm{Pr}_{2} \mathrm{C}_{6} \mathrm{H}_{3}\right)_{2}\right\} \mathrm{C}_{6} \mathrm{H}_{3}$ with divalent group 14 element amides featuring the parent $-\mathrm{NH}_{2}$ group. ${ }^{[137]}$ To date, however, no examples of monomeric stable divalent group 14 element amides featuring the parent $-\mathrm{NH}_{2}$ group have been reported. A number of stable $-\mathrm{NH}_{2}$ derivatives of silicon(IV) are known, ${ }^{[138-142]}$ and there are also a few stable $-\mathrm{NH}_{2}$ derivatives of germanium(IV) reported. ${ }^{[139,143,144]}$ These M(IV) compounds were obtained with the use of bulky coligands which prevent self-association by elimination of $\mathrm{NH}_{3}$. However these results have not been expanded so much to divalent derivatives. In recent years, we were successful in the preparation of compounds with the parent $-\mathrm{NH}_{2}$ group of group 13 elements such as $\mathrm{LAl}\left(\mathrm{NH}_{2}\right)_{2},{ }^{[145]} \mathrm{LGa}\left(\mathrm{NH}_{2}\right)_{2}{ }^{[123]}$ where $\mathrm{L}=\mathrm{HC}(\mathrm{CMeNAr})_{2} ; \mathrm{Ar}=2,6-i \mathrm{Pr}_{2} \mathrm{C}_{6} \mathrm{H}_{3}$. Like tetravalent species, the scarcity of divalent $-\mathrm{NH}_{2}$ derivatives is mainly due to their tendency to condense with elimination of ammonia. This process is especially feasible in divalent compounds where the group 14 elements can carry only one other substituent which renders steric protection more difficult. 


\subsection{Direction of the Thesis Work}

The Sections 1.2-1.7 describe the importance of compounds with low valent group 14 elements silicon, germanium, and tin. The work described herein was aimed at achieving a facile and easy synthetic route for the preparation of compounds with low valent group 14 elements silicon, germanium, and tin starting from easily accessible precursor that is supported by the sterically encumbered $\beta$-diketiminate ligand. Based on these facts the objectives of the present work are

1. to synthesize divalent germanium and tin halides, especially tin(II) fluorides.

2. to develop methodologies for the synthesis of germanium(II) and tin(II) hydrides.

3. to explore the reactivity of the $H$ functionality on the germanium(II) and tin(II) hydride compounds for the reaction with unsaturated compounds.

4. to explore the reactivity of $\beta$-diketiminate ligands for the preparation of germanium(II) and tin(II) organocompounds and their reactivity towards unsaturated molecules.

5. to develop the new method for the synthesis of six-membered N-heterocyclic germylene.

6. to develop a new synthetic strategy for the preparation of mononuclear molecular hydroxides of tin(II) via an aqueous route.

7. to synthesize monomeric germanium(II) and tin(II)-amides bearing the parent $-\mathrm{NH}_{2}$ group.

8. to study the germanium(II) and tin(II) hydrides for the exchange of hydrogen towards fluorine. 


\section{Chapter 2}

\section{Syntheses and Characterization of the Germanium(II) and Tin(II) Halides}

From the experience of the synthesis of $\mathrm{LGeCl}^{[146]} \mathrm{LSnCl}^{[146]}$ and $\mathrm{LGeF}^{[28]}$ we reacted the $\beta$ diketiminate lithium salt $\mathrm{LLi}\left(\mathrm{OEt}_{2}\right)^{[146]}\left[\mathrm{L}=\mathrm{HC}(\mathrm{CMeNAr})_{2} ; \mathrm{Ar}=2,6-i \mathrm{Pr}_{2} \mathrm{C}_{6} \mathrm{H}_{3}\right]$ with $\mathrm{SnF}_{2}$ or $\mathrm{LSnCl}$ with $\mathrm{Me}_{3} \mathrm{SnF} .{ }^{[147]}$ From the first reaction we cannot get the desired product, and in the second case there is no complete conversion to the target product, even under different conditions. Thus LSnF (1) was prepared from the reaction of LSnMe and $\mathrm{LSnN}\left(\mathrm{SiMe}_{3}\right)_{2}$ respectively with $\mathrm{Me}_{3} \mathrm{SnF}$ in toluene. The reaction of one equiv of $\beta$-diketiminate lithium salt $\mathrm{LLi}\left(\mathrm{OEt}_{2}\right)\left[\mathrm{L}=\mathrm{HC}(\mathrm{CMeNAr})_{2} ; \mathrm{Ar}=2,6-i \mathrm{Pr}_{2} \mathrm{C}_{6} \mathrm{H}_{3}\right]$ with either $\mathrm{GeBr}_{2}, \mathrm{SnBr}_{2}$, or $\mathrm{SnI}_{2}$ in diethyl ether affords the synthetically useful monomeric $\beta$-diketiminate-metal halides $\mathrm{LGeBr}$ (2), $\operatorname{LSnBr}(\mathbf{3})$, and LSnI (4) respectively.

\subsection{Synthesis of Tin(II) Fluoride}

Generally organotin(IV) fluorides are prepared by the substitution of chloride with KF in the presence of a phase transfer catalyst. ${ }^{[55-58]}$ Therefore the known compound LSnCl was used as a precursor. Compound $\mathrm{LSnCl}$ is soluble in common organic solvents. A complete reaction of $\mathrm{LSnCl}$ with $\mathrm{Me}_{3} \mathrm{SnF}$ in toluene even after refluxing for several days was not observed, although $\mathrm{LGeF}$ was obtained by the reaction of $\mathrm{LGeCl}$ with $\mathrm{Me}_{3} \mathrm{SnF} .{ }^{[28]}$ Consequently we changed the method for the preparation of compound LSnF. It is well known that $\mathrm{Me}_{4} \mathrm{Sn}$ and $\mathrm{Cl}_{4} \mathrm{Sn}$ dependent on the molecular ratio and temperature show metathesis and give in high yield $\mathrm{Me}_{3} \mathrm{SnCl}, \mathrm{Me}_{2} \mathrm{SnCl}_{2}$, and $\mathrm{MeSnCl}_{3}$ respectively. ${ }^{[148,149]}$ The question was whether it is possible to apply this type of reaction to $\mathrm{Me}-\mathrm{Sn}(\mathrm{II})$ and $\mathrm{F}-\mathrm{Sn}(\mathrm{IV})$ species. It was therefore reasonable to prepare $\mathrm{LSnMe}$ from $\mathrm{LSnCl}$ with $\mathrm{MeLi}$. Furthermore we prepared $\mathrm{LSnN}\left(\mathrm{SiMe}_{3}\right)_{2}$ and studied the reaction with $\mathrm{Me}_{3} \mathrm{SnF}$. Treatment of LSnMe with $\mathrm{Me}_{3} \mathrm{SnF}$ in toluene at room temperature, refluxing the suspension for $3 \mathrm{~h}$ afforded the corresponding fluoride LSnF (1) in high yield (85\%) (Scheme 2.1).

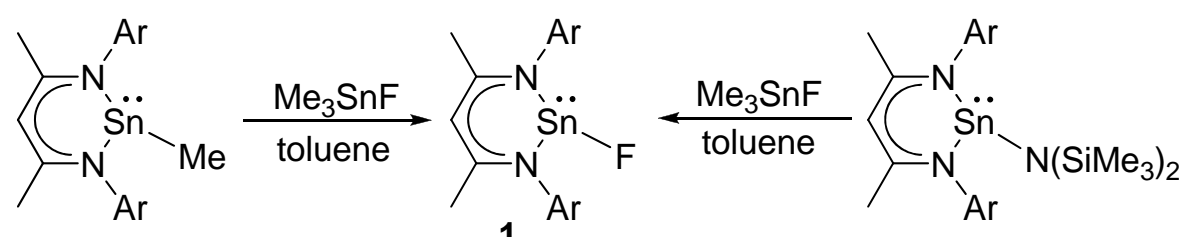

1

$$
\mathrm{Ar}=2,6-i \mathrm{Pr}_{2} \mathrm{C}_{6} \mathrm{H}_{3}
$$

Scheme 2.1. Preparation of compound 1 
Compound $\mathbf{1}$ is thermally stable. No decomposition was observed at temperature below the corresponding melting point $\left(130{ }^{\circ} \mathrm{C}\right)$ under an inert atmosphere. Compound $\mathbf{1}$ is also obtained by treating $\mathrm{LSnN}\left(\mathrm{SiMe}_{3}\right)_{2}$ with $\mathrm{Me}_{3} \mathrm{SnF}$ under the same conditions (Scheme 2.1). Compound $\mathbf{1}$ is a white solid that is soluble in benzene, toluene, $n$-hexane, and THF, in contrast to the fluorinating agent $\mathrm{Me}_{3} \mathrm{SnF}$, which is only slightly soluble in these solvents. $\mathbf{1}$ was characterized by multinuclear NMR spectroscopy, EI mass spectrometry, elemental analysis, and X-ray structural analysis. The ${ }^{1} \mathrm{H}$ NMR spectrum of compound 1 shows a singlet $(\delta 4.99 \mathrm{ppm})$ for the $\gamma-\mathrm{CH}$ proton and two septets $(\delta 3.84$ and $3.09 \mathrm{ppm})$ corresponding to the two different types of $\mathrm{CH}$ protons of the $i \mathrm{Pr}$ moieties. Also, the complete disappearance of the resonance arising from $\mathrm{Sn}-\mathrm{CH}_{3}(\delta 0.59 \mathrm{ppm})$ in LSnMe and the resonance arising from $\mathrm{Sn}-\mathrm{N}\left(\mathrm{Si}\left(\mathrm{CH}_{3}\right)_{3}\right)_{2}(\delta 0.46$ and $0.24 \mathrm{ppm})$ in $\mathrm{LSnN}\left(\mathrm{SiMe}_{3}\right)_{2}$ clearly indicates the formation of compound 1. The ${ }^{19} \mathrm{~F}$ NMR spectrum of $\mathbf{1}$ shows a singlet at $125.29 \mathrm{ppm}$, which is comparable with the analogues terminal ${ }^{19} \mathrm{~F}$ NMR resonance for LGeF $(\delta-113 \mathrm{ppm}) .{ }^{[28]}$ Also the ${ }^{119} \mathrm{Sn}$ NMR spectrum exhibits a doublet resonance $(\delta-371.52 \mathrm{ppm})$. This value is very much different from the precursor LSnMe $(\delta 192.65 \mathrm{ppm})$, but this was expected when compared with that of compound $\mathrm{LSnCl}(\delta-224 \mathrm{ppm})$. The $\mathrm{Sn}-\mathrm{F}$ coupling constant of $J\left({ }^{119} \mathrm{Sn}-{ }^{19} \mathrm{~F}\right)=3100 \mathrm{~Hz}$, is quite large, when compared with the previously reported Sn-F coupling constant. ${ }^{[51]}$ EI-MS of $\mathbf{1}$ gave the corresponding monomeric molecular ion peak $\mathrm{M}^{+}$.

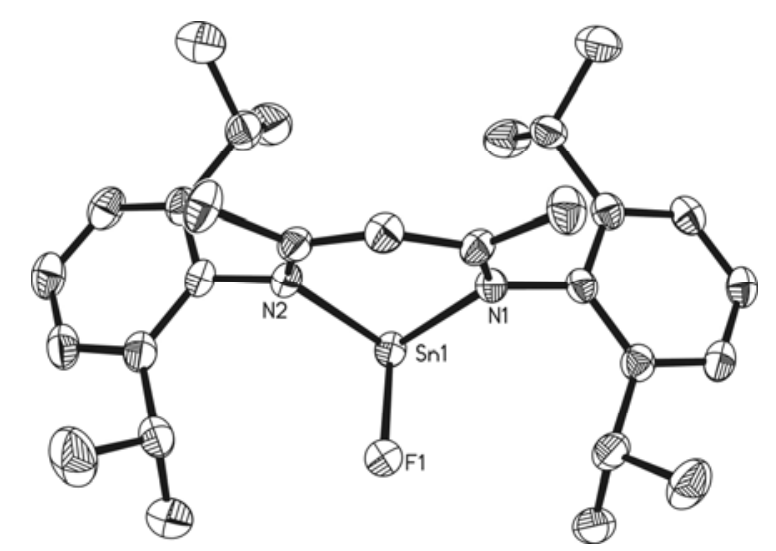

Figure 2.1. Molecular structure of 1 . Selected bond lengths $[\AA]$ and angles $\left[{ }^{\circ}\right]$; anisotropic displacement parameters are depicted at the $50 \%$ probability level and all restrained refined hydrogen atoms are omitted for clarity: Sn1-F1 1.988(2), Sn1-N1 2.178(3), N1-C13 1.333(4); N1-Sn1-F1 90.26(9), N2-Sn1-F1 90.20(9), N1Sn1-N2 86.22(10). 
The molecular structure of $\mathbf{1}$ has been determined by single crystal X-ray diffraction analysis (Figure 2.1) which demonstrates that the complex exists as a monomer. Colorless crystals of 1 suitable for single crystal X-ray analysis were obtained from a $n$-hexane solution at $-30{ }^{\circ} \mathrm{C}$ after one day. Compound 1 crystallizes in the monoclinic space group $P 2_{1} / c$, with one monomer in the asymmetric unit. The coordination polyhedron around the tin atom features a distorted tetrahedral geometry with one lone pair. The sum of angles around the tin atom is $266.68^{\circ}$. The terminal Sn-F bond distance (1.988 $\AA$ ) in $\mathbf{1}$ is quite short when compared with that of the bridging one of Sn-F-Sn $(2.156 \AA) .{ }^{[51]}$

\subsection{Synthesis of Germanium(II) and Tin(II) Bromides}

Reaction of the $\beta$-diketiminato lithium salt $\mathrm{LLi}\left(\mathrm{OEt}_{2}\right)$ with $\mathrm{GeBr}_{2}$ and $\mathrm{SnBr}_{2}$ in diethyl ether provided the monomeric compounds $\mathrm{LMBr}(\mathrm{M}=\mathrm{Ge},(\mathbf{2}) ; \mathrm{M}=\mathrm{Sn},(\mathbf{3}))$ with three-coordinate metal centers (Scheme 2.2).

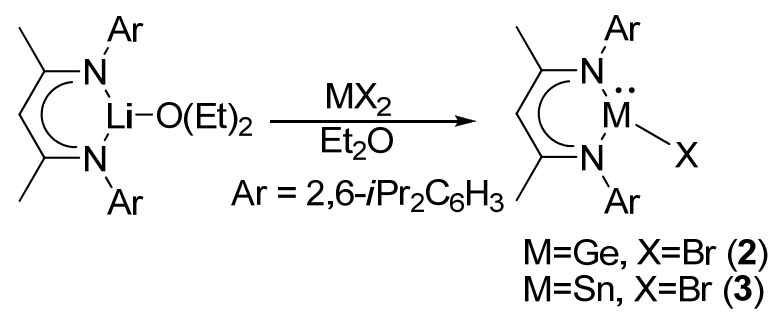

Scheme 2.2. Preparation of compounds 2 and 3

$\mathbf{2}$ and $\mathbf{3}$ are well soluble in common organic solvents, including benzene, THF, $n$-hexane, and n-pentane. $\mathbf{2}$ and $\mathbf{3}$ are characterized by NMR spectroscopy, EI-mass spectrometry, and elemental analysis. The ${ }^{119} \mathrm{Sn}$ NMR of $\mathbf{3}$ exhibits a singlet at $-180.47 \mathrm{ppm}$ (see Table 2.1 ).

The molecular structures of $\mathbf{2}$ and $\mathbf{3}$ are shown in Figures 2.2 and 2.3. The X-ray singlecrystal structures of $\mathbf{2}$ and $\mathbf{3}$ show that all compounds are monomeric. The metal centers adopt similar three-coordinate sites and reside in a distorted-tetrahedral environment with one vertex occupied by a electron lone pair. The structural feature of compounds $\mathbf{2}$ and $\mathbf{3}$ is similar to the corresponding chloro derivative. ${ }^{[146]}$ In compounds $\mathbf{2}$ and $\mathbf{3}$ the backbone of the chelating ligand is essentially planar and the metal atom is arranged out of the plane $\left(23.5^{\circ}\right.$ in 2 and $23.7^{\circ}$ in 3). However the bond angle of $\mathrm{N}(1)-\mathrm{Ge}(1)-\mathrm{N}(2)\left(90.80(6)^{\circ}\right)$ in 2 is larger than the corresponding angle in $3\left(\mathrm{~N}(1)-\mathrm{Sn}(1)-\mathrm{N}(2) 85.33(5)^{\circ}\right)$, and the bond length of $\mathrm{N}-\mathrm{Ge}$ (1.9824(14) and 1.9934(14) $\AA$ ) in 2 is slightly shorter than the corresponging N-Sn bond length in 3 (2.1758(13) and 2.1883(13) $\AA$ ), as expected. This indicates that the ionic 
interactions of the central metal atom with the ligand decrease from Ge to $\mathrm{Sn}$ due to the difference of the atomic radii (Ge(II) $0.93 \AA, \mathrm{Sn}(\mathrm{II}) 1.12 \AA$ )

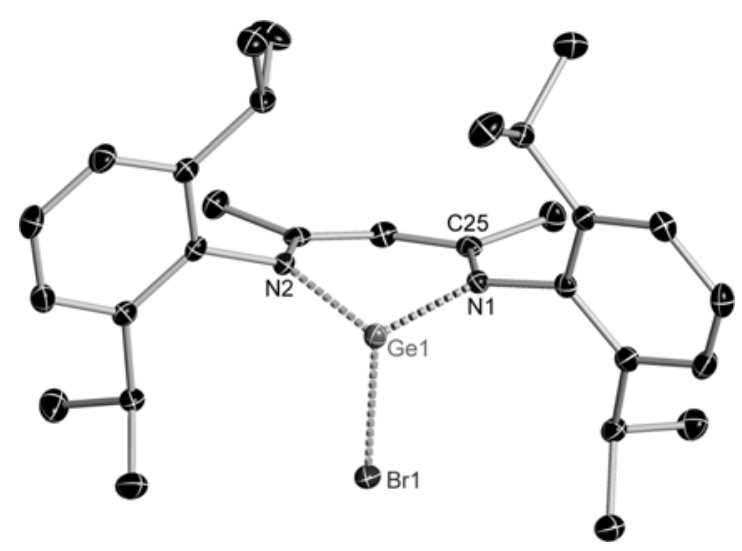

Figure 2.2. Molecular structure of 2. Anisotropic displacement parameters are depicted at the $50 \%$ probability level and all restrained refined hydrogen atoms are omitted for clarity. Selected bond lengths $[\AA]$ and angles $\left[{ }^{\circ}\right]$ : Ge1-Br1 2.5064(3), Ge1-N1 1.9934(14), N1-C25 1.326(2); N1-Ge1-N2 90.80(6), N1-Ge1-Br1 92.58(4), Ge1-N1-C25 124.91(11).

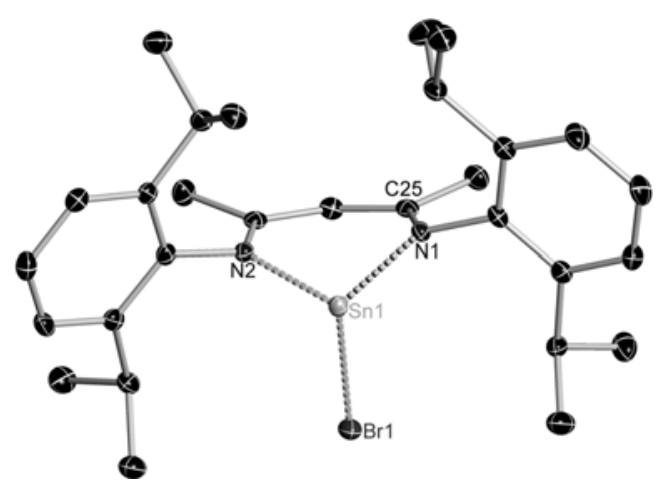

Figure 2.3. Molecular structure of 3. Anisotropic displacement parameters are depicted at the $50 \%$ probability level and all restrained refined hydrogen atoms are omitted for clarity. Selected bond lengths $[\AA]$ and angles $\left[^{\circ}\right]$ : Sn1-Br1 2.6373(2), Sn1-N1 2.1758(13), N1-C25 1.337(2); N1-Sn1-N2 85.33(5), N1-Sn1-Br1 93.35(3), Sn1N1-C25 123.82(10).

\subsection{Synthesis of Tin(II) Iodide}

The reaction of the $\beta$-diketiminato lithium salt $\mathrm{LLi}\left(\mathrm{OEt}_{2}\right)$ with $\mathrm{SnI}_{2}$ in diethyl ether provided the monomeric complex LSnI (4), (Scheme 2.3). Maintaing a diethyl ether solution of 4 for one day at $-32{ }^{\circ} \mathrm{C}$ resulted in colorless crystals suitable for X-ray structural analysis. Compound 4 crystallizes in the triclinic space group $P-1$, with one monomer in the asymmetric unit. The coordination environment of the central Sn atom exhibits a distorted tetrahedral geometry. 


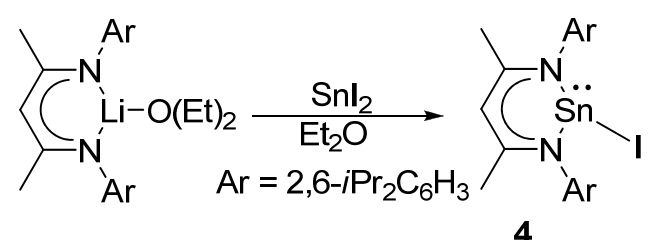

Scheme 2.3. Preparation of compound 4

For 4, two separate domains in the diffraction pattern were identified and integrated. The structure was refined as non-merohedrally twinned $(\mathrm{BASF}=0.228)$. Therefore a discussion of selected bond lengths or angles is not carried out.

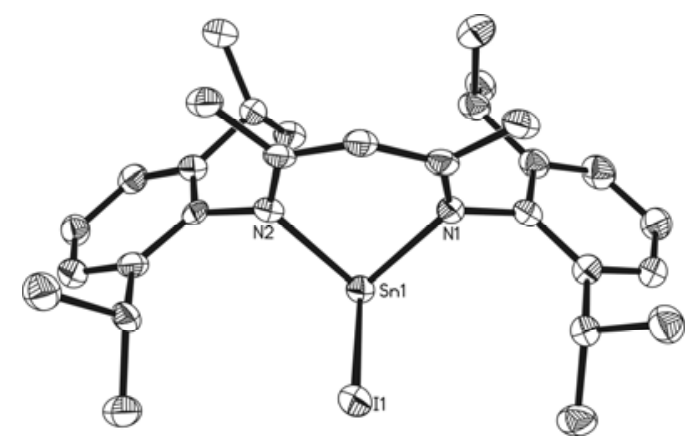

Figure 2.4. Molecular structure of 4

In the ${ }^{119} \mathrm{Sn}$ NMR spectrum the resonance of compound 4 arises at $\delta-107.33 \mathrm{ppm}$, which is very different from that of the terphenyl tin(II)iodide 2,6-Trip ${ }_{2} \mathrm{C}_{6} \mathrm{H}_{3} \mathrm{SnI}$, Trip $=2,4,6$ $i \operatorname{Pr}_{3} \mathrm{C}_{6} \mathrm{H}_{2} ;(\delta 1140 \mathrm{ppm}) .{ }^{[150]}$ The different chemical shifts might be due to the different coordination environment around both the tin(II) atoms. In Table 2.1 we summarise the ${ }^{119} \mathrm{Sn}$ NMR data of all four halogen derivatives of tin(II) compounds with the same $\beta$-diketiminate ligand. These data are in the expected range for the high field fluorine compound and low field iodine derivative. 
Table 2.1. ${ }^{119} \mathrm{Sn}$ NMR data of $\mathrm{Sn}(\mathrm{II})$ compounds

\begin{tabular}{|c|c|}
\hline Compound $^{*}$ & ${ }^{119} \operatorname{Sn} \operatorname{NMR}(\delta, \mathrm{ppm})$ \\
\hline LSnF (1) & -371.52 \\
\hline LSnCl & -224 \\
\hline LSnBr (3) & -180.47 \\
\hline LSnI (4) & -107.33 \\
\hline
\end{tabular}

$* \mathrm{~L}=\mathrm{HC}(\mathrm{CMeNAr})_{2} ; \mathrm{Ar}=2,6-i \mathrm{Pr}_{2} \mathrm{C}_{6} \mathrm{H}_{3}$ 


\section{Chapter 3}

\section{Germanium(II) Hydride an Effective Reagent for Hydrogermylation Reactions}

Metal hydrides and their complexes are considered valuable synthons in chemistry. It was demonstrated that main-group and transition-metal hydrides are important intermediates in some industrial processes and also function as catalysts. ${ }^{[59-67]}$ Furthermore, in the quest for alternative energy sources, metal hydrides have been considered as potential feedstocks for hydrogen storage. ${ }^{[68-71]}$ In recent years the chemistry of stable heteroleptic metal hydrides has attracted much attention due to their versatile reactivity. ${ }^{[72,151,152]}$ In comparison with main group hydrides the chemistry of transition metal hydrides is well documented. ${ }^{[153]}$ Organometallic hydrides of group 14 play an important role in various metathesis reactions and therefore the reactivity of hydrides like $\mathrm{R}_{3} \mathrm{SiH}, \mathrm{R}_{3} \mathrm{GeH}$, and $\mathrm{R}_{3} \mathrm{SnH}$ are well studied. ${ }^{[154]}$ Till now there is no reaction reported using germanium(II) hydride for hydrogenation reactions. The main reason for this imbalance is the synthetic difficulty and the stability of these low valent group 14 hydrides. In 2001 our group reported the first four coordinate monomeric germanium(II) hydride which exists as an adduct with the Lewis acid $\mathrm{BH}_{3}{ }^{\left[{ }^{[28]} \text { In }\right.}$ 2003 Power et al. reported the first isomeric germanium(II) hydride with a bulky aryl ligand. ${ }^{[81]}$ Recently, our group first published the molecular structure of the unique stable three coordinate germanium(II) hydride, $\mathrm{LGeH}, \quad\left[\mathrm{L}=\mathrm{HC}(\mathrm{CMeNAr})_{2} ; \mathrm{Ar}=2,6-i \mathrm{Pr}_{2} \mathrm{C}_{6} \mathrm{H}_{3}\right]$; (5) with the bulky $\beta$-diketiminate ligand. ${ }^{[79]}$ The corresponding carbon and silicon analogues are not reported yet. The unusual species LGeH (5) led us to explore the reactivity pattern of the germylene hydrogen bond. Herein, we report the first reaction of $\mathrm{LGeH}$ (5) with carbon dioxide, ketones, alkines, diazene, nitrous oxide, elemental sulfur, trimethylsilyl azide, and frustrated carbene borane Lewis pair respectively. To our surprise the formal oxidation state (II) at the germanium atom persists during the transfer of the hydrogen and germanium moiety from $\mathrm{LGeH}(5)$ to the corresponding carbon oxygen double bond and to the carbon carbon triple bond for the case of compounds with carbonyl group and alkine group respectively.

\subsection{Germylene Hydride Starting Material}

Initially we reported the synthesis of germanium(II) hydride, LGeH (5) from the reaction of the corresponding germanium(II) chloride, $\mathrm{LGeCl}^{[146]}$ with one equiv of $\mathrm{AlH}_{3} \cdot \mathrm{NMe}_{3}{ }^{[155]}$ in toluene. ${ }^{[79]}$ Preparation of 5 by using $\mathrm{AlH}_{3} \cdot \mathrm{NMe}_{3}$ resulted in the formation of the expected product contaminated with small amounts of the starting material $\mathrm{LGeCl}$. This slightly 
impure LGeH (5) leads to products containing chlorine, when it was used in hydrogermylation reactions. Therefore the method of preparation had to be improved. Treatment of $\mathrm{LGeCl}$ with potassium trisecbutylborohydride $\left(\mathrm{K}\left[\mathrm{HB}(\sec \mathrm{Bu})_{3}\right]\right)$ in toluene at $10{ }^{\circ} \mathrm{C}$ afforded the germanium(II) hydride (5) in high yield (82\%) (Scheme 3.1). The crude reaction product was recrystallized from $n$-hexane and obtained as pure red crystals which were investigated by ${ }^{1} \mathrm{H}$ NMR spectroscopy and well matches with the previously reported data. ${ }^{[79]}$

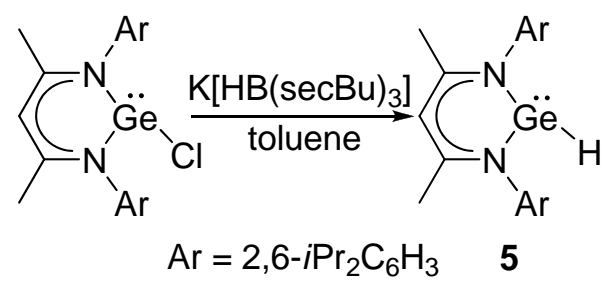

Scheme 3.1. Preparation of compound 5

\subsection{Reaction with Carbon Dioxide}

Reactions of metal hydrides with carbon dioxide to generate the metal formates are quite well known for transition metal hydrides and alkali metal hydrides. ${ }^{[156]}$ For group 14 metal hydrides there are only a few reports on the hydrogenation of carbon dioxide using silicon(IV) hydride and tin(IV) hydride, respectively. ${ }^{[157,158]}$ The reaction of carbon dioxide and silicon(IV) hydride requires a transition metal catalyst. ${ }^{[157(a)]}$ To the best of our knowledge there are no reports on the hydrogenation of carbon dioxide using low valent group 14 metal hydrides. Herein, we report on the synthesis of the germylene ester of formic acid $\mathrm{LGe}-\mathrm{O}-\mathrm{C}(\mathrm{O}) \mathrm{H}(\mathbf{6})$ by the reaction of 5 with carbon dioxide at room temperature without any catalyst in quantitative yield (Scheme 3.2).

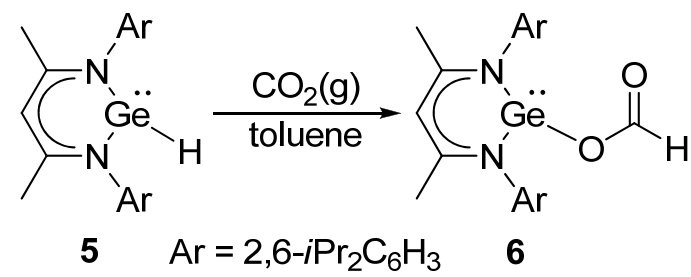

Scheme 3.2. Preparation of compound 6 
6 is a yellow solid, soluble in benzene, THF, $n$-hexane, and $n$-pentane and shows no decomposition on exposure to air. The compound was thoroughly characterized by ${ }^{1} \mathrm{H}$ NMR spectroscopy, IR spectroscopy, EI mass spectrometry, elemental analysis, and X-ray structural analysis. The ${ }^{1} \mathrm{H}$ NMR spectrum of 6 exhibits a singlet $(\delta 8.64 \mathrm{ppm})$ which can be assigned to the $\mathrm{CH}$ proton. Moreover, the ${ }^{1} \mathrm{H}$ NMR spectrum shows a singlet ( $\left.\delta 5.06 \mathrm{ppm}\right)$ for the $\gamma$-CH proton and two septets $(\delta 3.53$ and $3.08 \mathrm{ppm})$ corresponding to the two different $\mathrm{CH}$ protons of the $i \operatorname{Pr}$ moieties. The complete absence of the resonance $(\delta 8.08 \mathrm{ppm})$ arising from the hydride proton in $\mathrm{LGeH}(5)$ clearly indicates the formation of $\mathbf{6}$. The IR spectrum shows two bands at 2854 and $1690 \mathrm{~cm}^{-1}$, which are assigned to the $\mathrm{C}-\mathrm{H}$ and $\mathrm{C}=\mathrm{O}$ stretching frequencies.

Colorless crystals of $\mathbf{6}$ can be obtained after storage of a saturated $n$-hexane solution at -32 ${ }^{\circ} \mathrm{C}$ for one day. 6 crystallizes in the triclinic space group $P-1$ with one monomer in the asymmetric unit. The coordination polyhedron around the germanium atom features a distorted tetrahedral geometry with a stereochemical active lone pair (Figure 3.1).

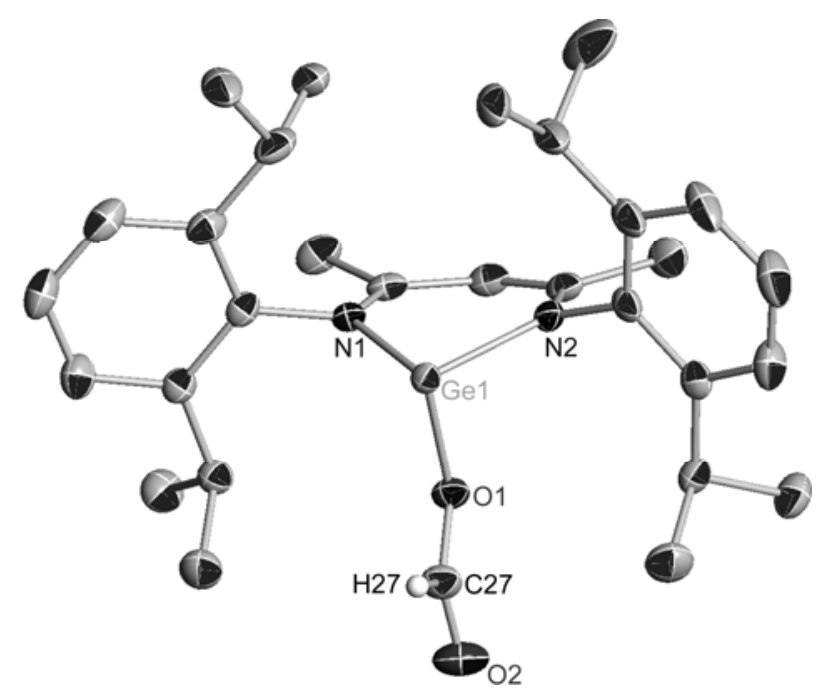

Figure 3.1. Anisotropic displacement parameters, depicted at the $50 \%$ probability level of 6 . Hydrogen atoms except that for the $\mathrm{C}(\mathrm{O}) \mathrm{H}$ group are omitted for clarity. Two of the $i$ Pr-groups are disordered. Selected bond lengths $[\AA]$ and angles $\left[{ }^{\circ}\right]$ : Ge1-O1 1.9339(18), O1-C27 1.304(3), C27-O2 1.204(3), Ge1-N1 1.969(2), Ge1N2 1.968(2); Ge1-O1-C27 120.07(17), O1-C27-O2 125.1(3), N2-Ge1-N2 91.34(9).

\subsection{Reactions with Ketones}

$\mathrm{LGeH}$ reacts with carbon dioxide, therefore we thought that it can react with other carbonyl compounds, which might generate the germanium(II)alkoxide by nucleophilic addition reaction to the carbon oxygen double bond. Although $\mathbf{5}$ displayed no reactivity toward 
acetone or benzophenone at room temperature, it reacts cleanly with activated ketones namely 2,2,2-trifluoro acetophenone and 2,2,2-trifluoroacetothiophane. Treatment of 5 with 2,2,2-trifluoro acetophenone and 2,2,2-trifluoroacetothiophane leads quantitatively to the germylene alkoxides 7 and $\mathbf{8}$ respectively with a Ge(II)-O-CH framework that is formed by nucleophilic hydride addition to the respective carbon of the carbonyl group (Scheme 3.3). Compounds 7 and 8 were monitored by ${ }^{1} \mathrm{H}$ NMR spectra. Sharp resonances in the ${ }^{1} \mathrm{H}$ NMR spectra of $\mathbf{7}$ and $\mathbf{8}$ gave the initial indication that the products have been formed in high yield. Compounds 7 and $\mathbf{8}$ have one $\mathrm{CF}_{3}$ group each and both display an interesting NMR spectrum. The ${ }^{1} \mathrm{H}$ NMR spectra of 7 and $\mathbf{8}$ exhibit a quartet each ( $\delta 4.73$ and $\left.5.04 \mathrm{ppm}\right)$ which corresponds to the quaternary $\mathrm{CH}$ proton and its coupling with the three $\mathrm{F}$-atoms

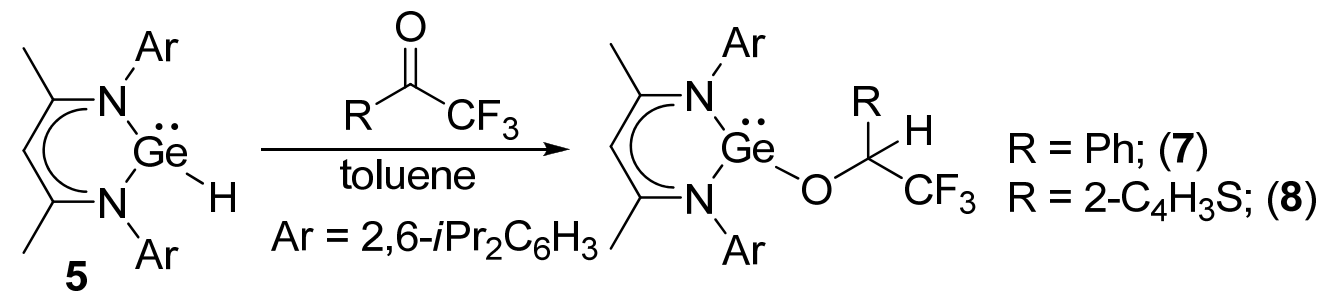

Scheme 3.3. Preparation of compounds 7 and 8

of the $\mathrm{CF}_{3}$ group $\left({ }^{3} J\left({ }^{19} \mathrm{~F}-{ }^{1} \mathrm{H}\right)=8\right.$ and $7 \mathrm{~Hz}$ respectively). The ${ }^{19} \mathrm{~F}$ NMR resonance arises as a doublet $(\delta-75.76(7)$ and $-76.29(8)$ ppm) with the same coupling constant of 8 and $7 \mathrm{~Hz}$ respectively. The four isopropyl groups of $\mathbf{7}$ and $\mathbf{8}$ are showing four different resonances, and even the two methyl groups in the backbone exhibit two different signals each in the ${ }^{1} \mathrm{H}$ NMR spectra.

Single crystals of $\mathbf{7}$ and $\mathbf{8}$ suitable for X-ray structural analysis were obtained from $n$-hexane solutions. 7 and 8 crystallize in the triclinic space group $P-1$ and monoclinic space group $P 2_{1} / c$ respectively. The molecular structures of 7 and $\mathbf{8}$ are shown in Figures 3.2 and 3.3. The asymmetric unit of $\mathbf{7}$ and $\mathbf{8}$ contains one formula unit of the compound, and there are two of the molecules in each unit cell. As predicted, based on the ${ }^{1} \mathrm{H}$ NMR spectrum and EI mass spectrum, compounds 7 and $\mathbf{8}$ contain a Ge(II)-O-CH core. The three coordinate germanium atom is surrounded by two $\mathrm{N}$ atoms of the $\beta$-diketiminato ligand, and an exocyclic $\mathrm{O}$ atom. The Ge-O bond lengths (1.862(3) $\AA, 1.855(2) \AA)$ are shorter when compared with that of 
compound 6, LGeOC(O)H (1.9339(18) A). The O-C bond distances of 7 and $\mathbf{8}$ are in a narrow range to each other $(1.407 \AA$ and $1.417 \AA)$.

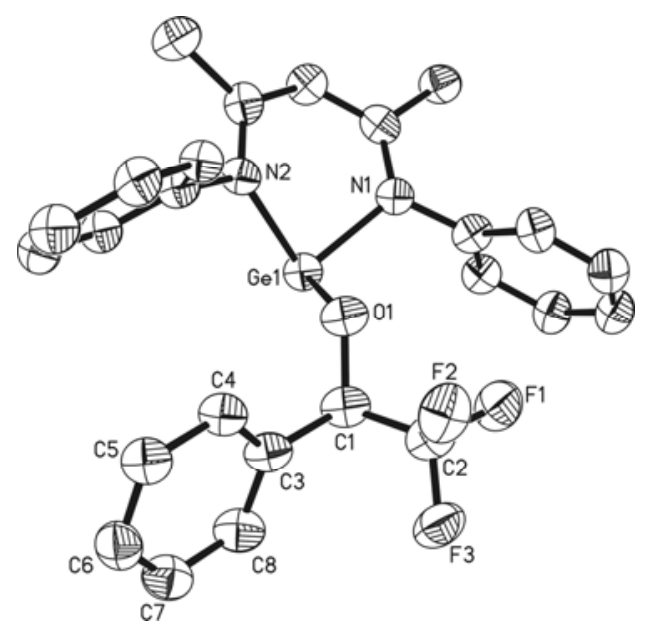

Figure 3.2. Molecular structure of 7. Anisotropic displacement parameters are depicted at the $50 \%$ probability level and all restrained refined hydrogen atoms are omitted for clarity. Selected bond lengths $[\AA]$ and angles $\left[{ }^{\circ}\right]$; Ge1-O1 1.862(3), Ge1-N1 2.002(4), O1-C1 1.407(6); N1-Ge1-N2 88.77(15), N1-Ge1-O1 97.52(15), Ge1O1-C1 112.8(3).

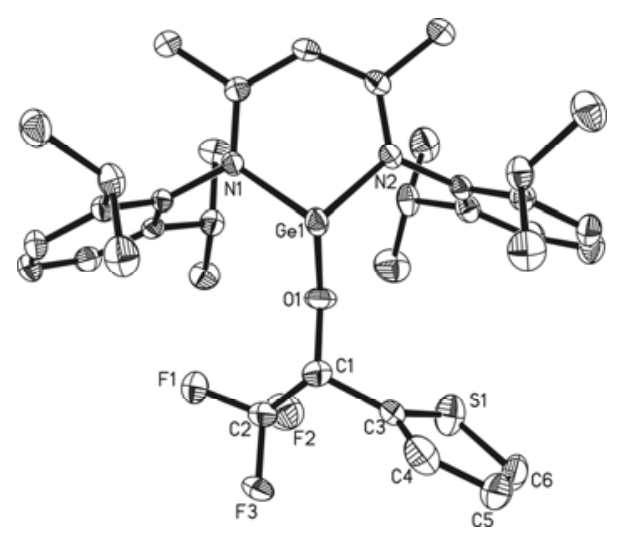

Figure 3.3. Molecular structure of 8. Anisotropic displacement parameters are depicted at the $50 \%$ probability level and all restrained refined hydrogen atoms are omitted for clarity. Selected bond lengths $[\AA]$ and angles $\left[{ }^{\circ}\right]$; Ge1-O1 1.855(2), Ge1-N1 2.011(3), O1-C1 1.417(4); N1-Ge1-N2 88.45(11), N1-Ge1-O1 96.69(11), Ge1O1-C1 112.8(2).

\subsection{Reactions with Compounds Containing $\mathrm{C} \equiv \mathrm{C}$ Triple Bonds}

The hydrogenation of unsaturated hydrocarbon compounds with $\mathrm{Ge}(\mathrm{IV})-\mathrm{H}$ reagents give a variety of acyclic and cyclic carbon-functionalized germanium compounds. ${ }^{[159]}$ Due to the variable polarity of the $\mathrm{Ge}-\mathrm{H}$ bond, various catalysts have been used in hydrogermane reactions to initiate different path ways. ${ }^{[160]}$ Herein, we report hydrogermylation reactions of alkines using germanium(II) hydride without any catalyst. The germanium(II) hydride (5) reacts with the alkines $\mathrm{HC} \equiv \mathrm{CCO}_{2} \mathrm{Me}, \mathrm{HC} \equiv \mathrm{CCO}_{2} \mathrm{Et}, \mathrm{MeO}_{2} \mathrm{CC} \equiv \mathrm{CCO}_{2} \mathrm{Me}, \mathrm{EtO}_{2} \mathrm{CC} \equiv \mathrm{CCO}_{2} \mathrm{Et}$, 
and $t \mathrm{BuO}_{2} \mathrm{CC} \equiv \mathrm{CCO}_{2} t \mathrm{Bu}$ at room temperature to form germanium(II) substituted alkenes 9, 10, 11, 12, and 13. Compounds 9 and 10 are obtained by the 1,2-addition of germylene hydride (5) to the alkine methyl and ethyl propiolate and the result is the transfer of the hydrogen atom and germylene across the carbon carbon triple bond (Scheme 3.4), rather than the elimination of $\mathrm{H}_{2}$ due to the different polarized $\mathrm{Ge}-\mathrm{H}$ and $\mathrm{C}-\mathrm{H}$ bonds.

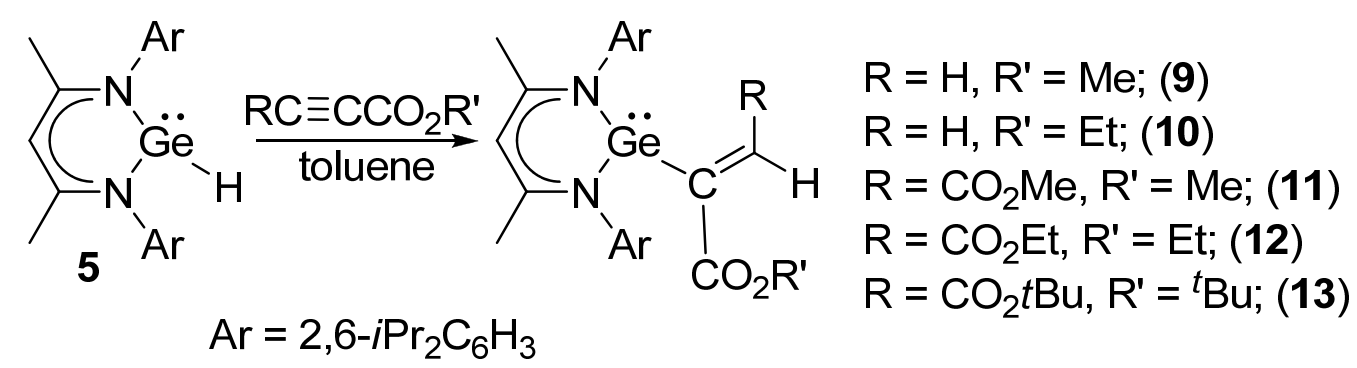

Scheme 3.4. Preparation of compounds 9, 10, 11, 12, and 13

The ${ }^{1} \mathrm{H}$ NMR spectra of $\mathbf{9}$ and $\mathbf{1 0}$ each exhibit two broad resonances $(\delta 6.20$ and $5.82 \mathrm{ppm}$ for 9 and $\delta 6.25$ and $5.85 \mathrm{ppm}$ for 10) which corresponds to the two alkenyl protons. Moreover, the ${ }^{1} \mathrm{H}$ NMR spectrum of $\mathbf{1 0}$ shows a quartet and a triplet resonance ( $\delta 4.07$ and $\left.0.87 \mathrm{ppm}\right)$ corresponding to the two different types of $\mathrm{CH}$ protons of the ethyl moieties. The IR spectrum of 10 exhibits bands at 3379 and $1723 \mathrm{~cm}^{-1}$, which are assigned to the $\mathrm{C}-\mathrm{H}$ and $\mathrm{C}=\mathrm{O}$ stretching frequencies.

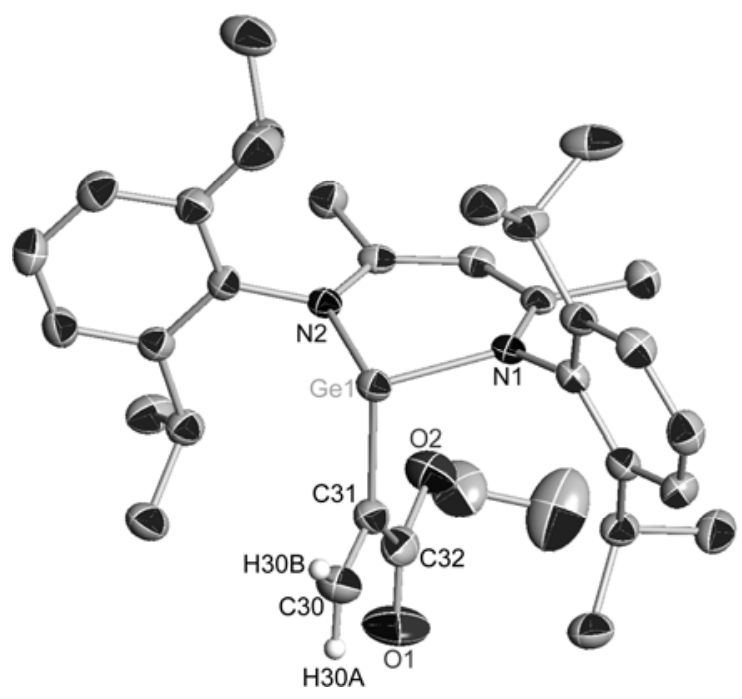

Figure 3.4. Anisotropic displacement parameters, depicted at the $50 \%$ probability level of $\mathbf{1 0}$. Hydrogen atoms except that for the $\mathrm{CH}_{2}$ group are omitted for clarity. Selected bond lengths $[\AA]$ and angles $\left[{ }^{\circ}\right]$ : Ge1-C31 2.033(3), C30-C31 1.324(4), C31-C32 1.491(4), C32-O1 1.200(4), C32-O2 1.337(4), Ge1-N1 2.002(2), Ge1N2 1.994(2); Ge1-C31-C32 130.5(2), C31-C32-O1 126.6(3), C31-C32-O2 111.2(2), N1-Ge1-N2 91.65(9). 
Compound 10 crystallizes in the monoclinic space group $P 2_{1} / c$, with one monomer in the asymmetric unit. Single crystals were obtained from a saturated $n$-hexane solution at $-32{ }^{\circ} \mathrm{C}$ after two days. The coordination polyhedron around the germanium atom features a distorted tetrahedral geometry with a stereochemical active lone pair (Figure 3.4).

Furthermore we were interested in the selectivity of the addition across the carbon carbon triple bond. Therefore we selected the disubstituted alkines, dimethyl acetylenedicarboxylate, diethyl acetylenedicarboxylate, and ditertiarybutyl acetylenedicarboxylate. 5 reacts with dialkyl acetylenedicarboxylate, $\left(\mathrm{RO}_{2} \mathrm{CC} \equiv \mathrm{CCO}_{2} \mathrm{R}, \mathrm{R}=\mathrm{Me}\right.$, Et, and $\left.t \mathrm{Bu}\right)$ in toluene at room temperature to form only the isomer with $E$-germylene substituted alkene (Scheme 3.4). This was confirmed by ${ }^{1} \mathrm{H}$ NMR in solution. The ${ }^{1} \mathrm{H}$ NMR spectrum shows a singlet resonance $(\delta$ $6.64,6.68$, and $6.65 \mathrm{ppm}$ ) which corresponds to the alkenyl protons of the germylene substituted alkenes 11, 12, and 13, respectively. In addition these compounds exhibit a resonance at 4.89, 4.91, and $4.96 \mathrm{ppm}$ for the $\gamma$-CH proton. Furthermore 11, 12, and $\mathbf{1 3}$ were characterized by X-ray structural analysis (Figures 3.5, 3.6, and 3.7). 11 crystallizes in the monoclinic space group $P 2_{1} / n$, with one monomer in the asymmetric unit from saturated $n$ hexane solution at $-32{ }^{\circ} \mathrm{C}$ after one day (Figure 3.5). In the solid state also the $E$ - form is the only isomer that was observed.

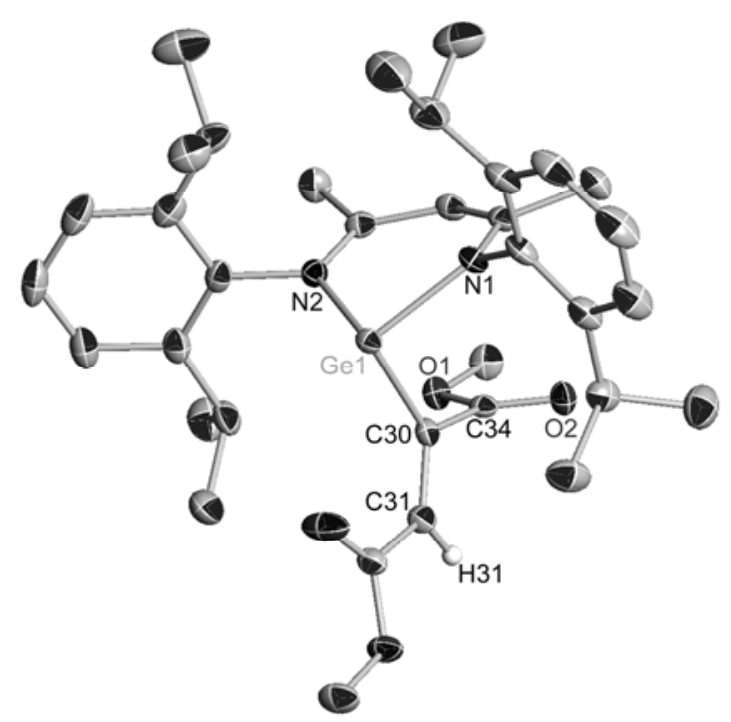

Figure 3.5. Anisotropic displacement parameters, depicted at the $50 \%$ probability level of $\mathbf{1 1}$. Hydrogen atoms except that for the $\mathrm{CH}$ group are omitted for clarity. Selected bond lengths $[\AA]$ and angles $\left[{ }^{\circ}\right]$ : Ge1-C30 2.1093(14), C30-C31 1.340(2), C30-C34 1.4920(19), C34-O1 1.3415(17), C34-O2 1.2131(18), Ge1-N1 2.0060(12), Ge1-N2 2.0190(12); Ge1-C30-C31 122.99(11), Ge1-C30-C34 123.07(10), C30-C34-O1 112.53(12), C30-C34-O2 124.28(13), N1-Ge1-N2 90.78(5). 
Compounds 12 and 13 crystallizes in the monoclinic space group $P 2_{1} / c$ and $P 2_{1} / n$ respectively (Figures 3.6 and 3.7). The coordination polyhedron around the germanium atom features a distorted tetrahedral geometry with one lone pair.

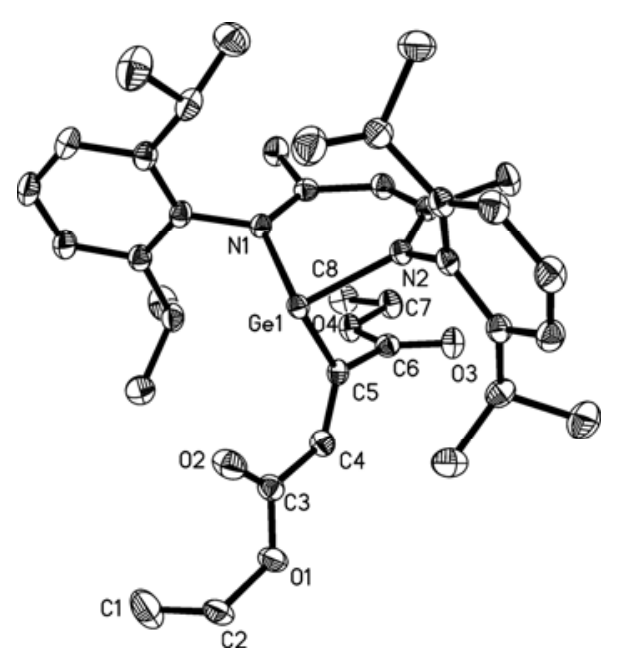

Figure 3.6. Molecular structure of 12. Anisotropic displacement parameters are depicted at the $50 \%$ probability level and all restrained refined hydrogen atoms are omitted for clarity. Selected bond lengths $[\AA]$ and angles $\left[^{\circ}\right]$; Ge1-C5 2.1083(18), Ge1-N1 2.0177(15), C4-C5 1.338(3); N1-Ge1-N2 91.07(6), N1-Ge1-C5 92.07(6), Ge1C5-C4 122.03(14).

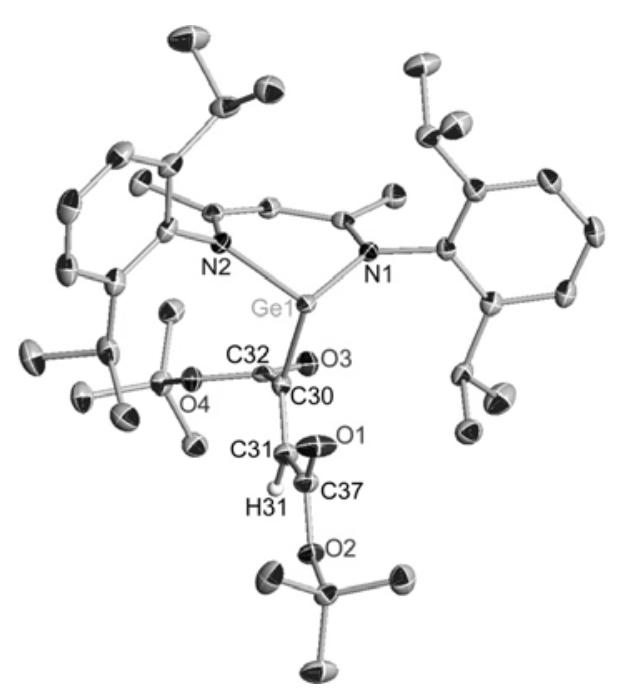

Figure 3.7. Anisotropic displacement parameters, depicted at the $50 \%$ probability level of $\mathbf{1 3}$. Hydrogen atoms except that for the $\mathrm{CH}$ group are omitted for clarity. Selected bond lengths $[\AA]$ and angles $\left[{ }^{\circ}\right]$ : $\mathrm{Ge} 1-\mathrm{C} 30$ 2.1060(16), C30-C31 1.340(2), C30-C37 1.500(2), C37-O3 1.2095(18), C37-O4 1.3491(18), Ge1-N1 2.0133(13), Ge1-N2 2.0175(13); Ge1-C30-C37 126.25(11), Ge1-C30-C31 121.37(11), C30-C37-O3 124.51(14), C30-C37-O4 110.68(12), N1-Ge1-N2 90.73(5). 


\subsection{Reactions with a Compound Containing a $\mathrm{N}=\mathrm{N}$ Double Bond}

Furthermore we performed the reaction of $\mathbf{5}$ with nitrogen nitrogen double bonded compound namely, diethyl azodicarboxylate (DEAD) to complete the reactivity pattern of $\mathrm{LGeH}(\mathbf{5})$ (Scheme 3.5). This reaction proceeds rapidly at room temperature under oxidative addition to give compound 14 in high yield.

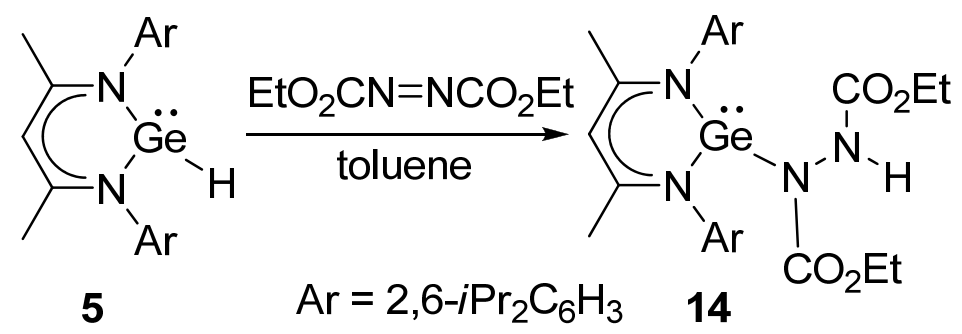

Scheme 3.5. Preparation of compound 14

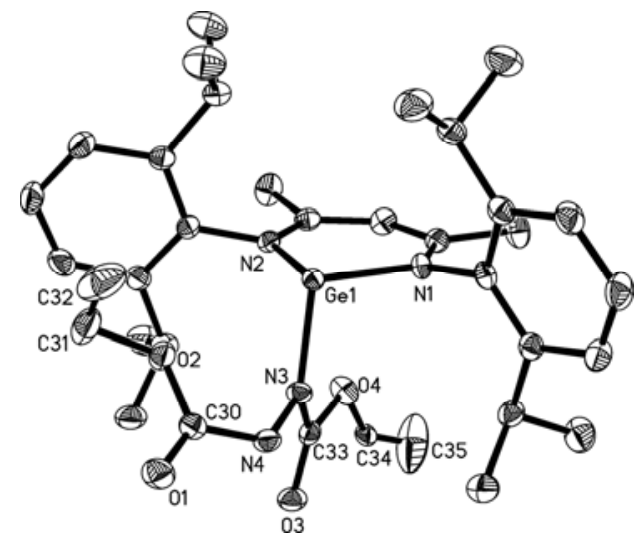

Figure 3.8. Molecular structure of 14. Thermal elipsoids are shown at $50 \%$ probability. $\mathrm{H}$ atoms are omitted for clarity reasons. Selected bond lengths $[\AA]$ and angles $\left[{ }^{\circ}\right]$ : Ge1-N1 2.009(2), Ge1-N3 1.990(2), N3-N4 1.418(14), N1-Ge1-N2 90.94(9), N1-Ge1-N3 98.93(9).

Compound 14 crystallizes in the monoclinic space group $P 2_{1} / n$, with one monomer in the asymmetric unit. Single crystals were obtained from a saturated $n$-hexane solution at $-32{ }^{\circ} \mathrm{C}$ after three days (Figure 3.8). In the solid state the $E$-form is the only isomer that was observed. The IR spectrum exhibits bands at 3245 and $1752 \mathrm{~cm}^{-1}$, which are assigned to the $\mathrm{N}-\mathrm{H}$ and $\mathrm{C}=\mathrm{O}$ stretching frequencies.

\subsection{Reactions with Nitrous Oxide}

Compounds with multiple bonds between elements of group 14 and group 16 are known. ${ }^{[161]}$ They are generally formed by the reaction of compounds with low valent 
group 14 elements with a chalcogen source. It is well known that nitrous oxide acts as a mono oxygen source. It can supply the oxygen to the low valent metal center or to the metal-metal bond to generate metal oxygen compounds. ${ }^{[162,163]}$ However there is no reaction known of group 14 metal hydride with nitrous oxide to form the metal hydroxide. Herein we describe the reaction of 5 with nitrous oxide with formation of $\mathrm{LGeOH}(\mathbf{1 5})$ in almost quantitative yield (Scheme 3.6). There is no indication of formation of $\mathrm{LGe}(\mathrm{O}) \mathrm{H}$ or $\mathrm{LGe}(\mathrm{O}) \mathrm{OH}$ even after a longer reaction time with nitrous oxide. Compound $\mathbf{1 5}$ was previously reported by our group by the reaction of $\mathrm{LGeCl}$ with water in the presence of $1,3-\operatorname{di}\left(2,4,6-\mathrm{Me}_{3} \mathrm{C}_{6} \mathrm{H}_{2}\right)-2$-ylidene. ${ }^{[127]}$

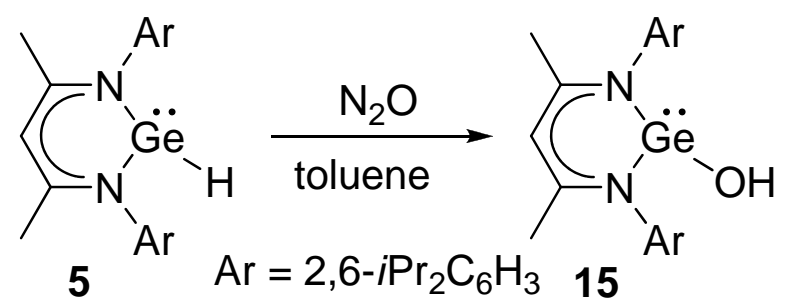

Scheme 3.6. Preparation of compound 15

\subsection{Reactions with Elemental Sulfur}

The insertion of elemental sulfur into the metal hydride bond or oxidative addition to a low valent metal center in the presence of transition metal catalysts is quite well known. ${ }^{[164]}$ In contrast, there are no reactions reported where germanium hydrides were used for the formation of germanium thiols by insertion of elemental sulfur. However, there are some reports on the preparation of germanium thiol using other routes. ${ }^{[165]}$ There are some additional reports of the synthesis of silane thiols by insertion of elemental sulfur into $\mathrm{Si}-\mathrm{H}$ bonds. ${ }^{[166]}$ The oxidative addition of elemental sulfur to germanium(II) atom is known. ${ }^{[167]}$ Herein, we report the simultaneous insertion and addition of elemental sulfur into the germylene hydrogen bond and to the germylene atom, respectively, at room temperature without any catalyst. The resulting compound $\mathbf{1 6}$ is the germanium analogue of dithio carboxylic acid with a Ge(S)SH moiety. To the best of our knowledge, there are only five further crystal structures deposited in the Cambridge Crystallographic Database ${ }^{[168]}$ with a $\mathrm{Ge}-\mathrm{S}-\mathrm{H}$ unit and only one derivative that contains a bridging $\mathrm{Ge}(\mathrm{S}) \mathrm{SH}$ moiety of composition $\left[\mathrm{Dmp}(\mathrm{Dep}) \mathrm{Ge}(\mu-\mathrm{S})(\mu-\mathrm{SH}) \mathrm{Ru}\left(\mathrm{PPh}_{3}\right)\right] \mathrm{OTf}{ }^{[169]}$ Therefore compound $\mathbf{1 6}$ is the first example of a dithio carboxylic acid with a heavier group 14 element and a terminal SH bond. 
The reaction of $\mathbf{5}$ in the presence of two equivalents of elemental sulfur at room temperature in toluene after $0.5 \mathrm{~h}$ leads to the white compound $\mathbf{1 6}$ in a moderate yield (60\%) (Scheme $3.7)$.

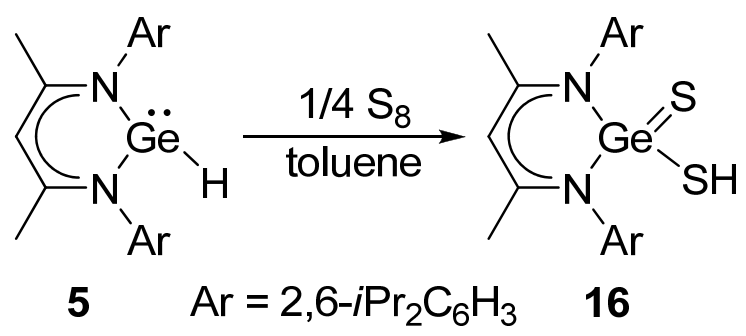

Scheme 3.7. Preparation of compound $\mathbf{1 6}$

16 is a colorless solid soluble in benzene, THF, $n$-hexane, and $n$-pentane, respectively and shows no decomposition on exposure to dry air. 16 was characterized by ${ }^{1} \mathrm{H}$ NMR and IR spectroscopy, EI mass spectrometry, elemental analysis, and X-ray structural analysis. The ${ }^{1} \mathrm{H}$ NMR spectrum of $\mathbf{1 6}$ showed a singlet $(\delta-0.1 \mathrm{ppm})$ which can be assigned to the $\mathrm{SH}$ proton by comparison with the Al-SH analogue. ${ }^{[170]}$ The IR spectrum exhibited a weak band at $2590 \mathrm{~cm}^{-1}$, and it is lower in wave numbers than the literature data for $\mathrm{H}_{2} \mathrm{~S}(\mathrm{~g})\left(2615 \mathrm{~cm}^{-}\right.$ $\left.{ }^{1}\right) .{ }^{[171]}$

Maintaining a toluene solution of $\mathbf{1 6}$ for two days at $-32{ }^{\circ} \mathrm{C}$ resulted in colorless single crystals suitable for X-ray structural analysis. 16 crystallizes in the monoclinic space group $P 2_{1} / n$, with one molecule of $\mathbf{1 6}$ and one solvent molecule of toluene in the asymmetric unit. 16 exists as a monomer in the solid state (Figure 3.9). There are no intermolecular hydrogen bonds observed in the crystal lattice. The coordination polyhedron around the germanium atom comprises two nitrogen atoms from the supporting ligand, one mercapto group, and one sulfur atom, featuring a distorted tetrahedral geometry. The Ge-N bond lengths and $\mathrm{N}-\mathrm{Ge}-\mathrm{N}$ angle are $1.9110(10), 1.9157(10) \AA$, and $95.79(4)^{\circ}$, respectively. The comparisons of Ge-N bond lengths in 5-8, 10-15 with those of $\mathbf{1 6}$ are quite different due to the different oxidation states of the germanium atoms. 


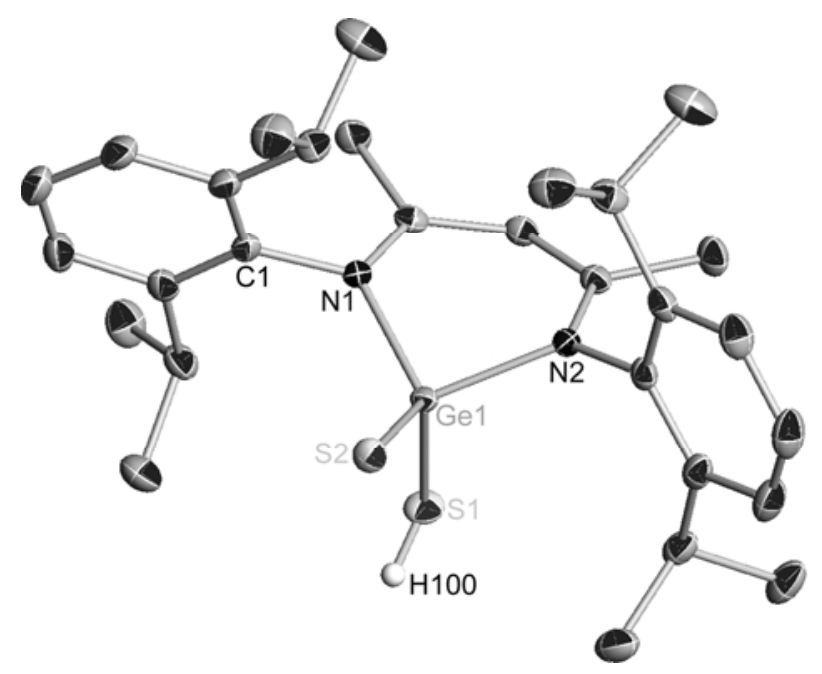

Figure 3.9. Anisotropic displacement parameters, depicted at the $50 \%$ probability level of 16. One toluene molecule and hydrogen atoms except that for the SH group are omitted for clarity. Selected bond lengths [ $]$ and angles [ $\left.{ }^{\circ}\right]$ : Ge1-S1 2.2422(4), Ge1-S2 2.0641(4), Ge1-N1 1.9157(10), Ge1-N2 1.9111(10); S1-Ge1-S2 119.485(16), S1-Ge1-N1 100.66(3), S2-Ge1-N2 116.10(3).

No evidence was found for any tautomeric equilibrium of $\mathbf{1 6}$ and consequently the two Ge-S bond lengths are very different with 2.0641(4) and 2.2421(4) $\AA$. The shorter one (Ge1-S2) shows a good agreement with the reported $\mathrm{Ge}=\mathrm{S}$ bond lengths in $\left[\left\{\mathrm{HC}(\mathrm{CMeNAr})_{2}\right\} \mathrm{Ge}(\mathrm{S}) \mathrm{X}\right]$ $(\mathrm{X}=\mathrm{Cl}, \mathrm{F},)^{[172]}(2.053(6)$, and 2.050(9) $\AA$ respectively), and can therefore be assigned to a formal double bond. Also the Ge1-S1 bond length is comparable with that previously reported for the Ge-SH moiety. ${ }^{[173]}$

The formation of $\mathbf{1 6}$ requires the oxidation of germanium(II) to germanium(IV). Therefore the synthesis of $\mathbf{1 6}$ also involves an unprecedented oxidative addition-insertion of elemental sulfur into the $\mathrm{Ge}(\mathrm{II})-\mathrm{H}$ bond, which leads to the formal conversion of the $\mathrm{GeH}$ hydride to a $\mathrm{SH}$ proton. We assume that the first step of the reaction necessitates the intermediate $\mathrm{LGe}(\mathrm{II}) \mathrm{SH}$, which can result from an insertion of sulfur into the germanium(II) hydride bond. The next step is the oxidative addition of elemental sulfur to LGe(II)SH. That leads to a concomitant $\mathrm{LGe}(\mathrm{S}) \mathrm{SH}$ formation. Alternatively it may be possible that first an oxidative addition of elemental sulfur to $\mathrm{LGe}(\mathrm{II}) \mathrm{H}$ might occur with the formation of $\mathrm{LGe}(\mathrm{S}) \mathrm{H}$. The insertion of sulfur into the $\mathrm{Ge}-\mathrm{H}$ bond leads then to an oxidation of the hydride to a proton under reduction of the sulfur to sulfide. The recently isolated LGe(II)OH supports as a first step the insertion of sulfur rather than the oxidation of germanium. Surprisingly there is no 
precedent reaction of group 14 known where sulfur is added and inserted simultaneously at the same element. We were not able to isolate any intermediate of this reaction.

\subsection{Reaction with Trimethylsilyl Azide}

In 2005 our group reported the first end-on azide insertion into an Al-C bond of an aluminacyclopropene and the formation of aluminaazacyclobutene. ${ }^{[174]}$ Subsequently we became interested in studying the reactivity of $\mathbf{5}$ with trimethylsilyl azide. Therefore we expected that the azide might also insert end-on into the germanium-hydrogen bond of 5 . However the reaction of $\mathbf{5}$ with trimethylsilyl azide at room temperature afforded two products in a ratio of $3: 1$ (Scheme 3.8 ).

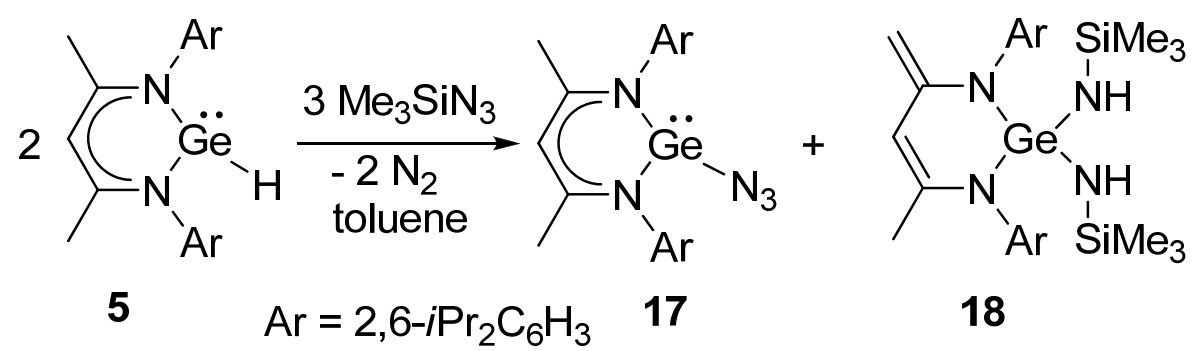

Scheme 3.8. Preparation of compounds 17 and 18

One is the germanium(II) azide (17), and the other is the germanium(IV) diamide (18). Compound 17 was formed by metathesis reaction of 5 with $\mathrm{Me}_{3} \mathrm{SiN}_{3}$ under elimination of $\mathrm{Me}_{3} \mathrm{SiH}$. Silicon(IV) and tin(IV) azides have been reported to be versatile reagents in maingroup element chemistry ${ }^{[175]}$ and in organic synthesis. ${ }^{[176]}$ In comparison, studies on germanium(IV) azides are still limited ${ }^{[177]}$ and for germanium(II) azides are to our knowledge only two compounds are known, ${ }^{[178,179]}$ probably due to their propensity to decompose to germanium(IV) nitrides. These two germanium(II) azides are prepared by nucleophilic substitution reactions using $\mathrm{NaN}_{3}$ and germanium(II) chloride precursors. Whereas $\mathbf{1 8}$ was formed by elimination of dinitrogen from azide, the resulting intermediate nitrene inserts into the germanium-hydrogen bond. The final oxidative addition of another nitrene is followed by simultaneous hydrogen transfer from one methyl group, which is attached to the heterocyclic ring backbone. A few reactions of group 14 compounds with divalent elements and organic azides have been reported. ${ }^{[180]}$ The initial dinitrogen elimination from $\mathrm{Me}_{3} \mathrm{SiN}_{3}$ is generally accepted, and supported by experimental observations. ${ }^{[181,182]}$ 
The formation of the imide $\mathrm{LGe}(\mathrm{F}) \mathrm{NSiMe}_{3}$ was observed by elimination of dinitrogen from $\mathrm{Me}_{3} \mathrm{SiN}_{3}$ followed by oxidative addition to the germanium(II) center of $\mathrm{LGeF},{ }^{[28]}$ whereas the LGeMe reacted with $\mathrm{Me}_{3} \mathrm{SiN}_{3}$ under dinitrogen elimination, oxidative addition, followed by intramolecular hydrogen transfer from one methyl group of the backbone to yield $\mathrm{L}^{\prime} \mathrm{Ge}(\mathrm{Me}) \mathrm{NHSiMe}_{3} .{ }^{[29]}$ Subsequently we propose a reasonable pathway for the formation of 16 (Scheme 3.9).

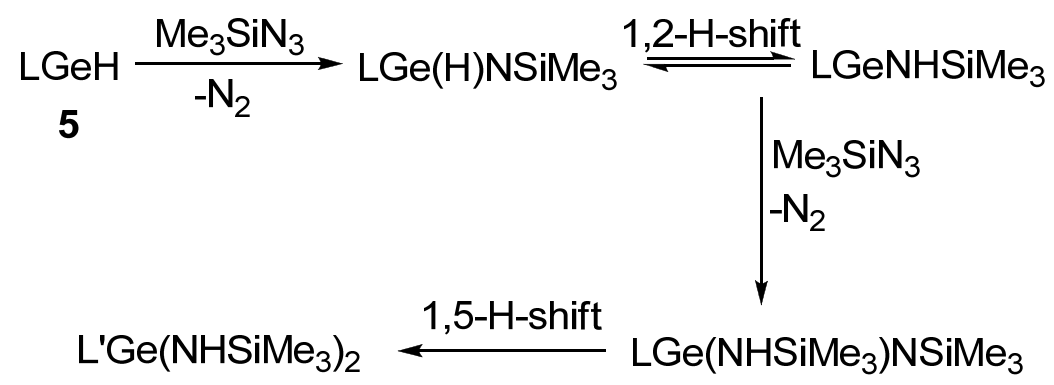

18

$$
\begin{gathered}
\mathrm{L}=\mathrm{HC}(\mathrm{CMeNAr})_{2} ; \mathrm{Ar}=2,6-\mathrm{iPr}_{2} \mathrm{C}_{6} \mathrm{H}_{3} \\
\mathrm{~L}^{\prime}=\mathrm{CH}\left\{\left(\mathrm{C}=\mathrm{CH}_{2}\right)(\mathrm{CMe})(\mathrm{NAr})_{2}\right\} ; \mathrm{Ar}=2,6-\mathrm{iPr}_{2} \mathrm{C}_{6} \mathrm{H}_{3}
\end{gathered}
$$

Scheme 3.9. Proposed pathway for the formation of 18

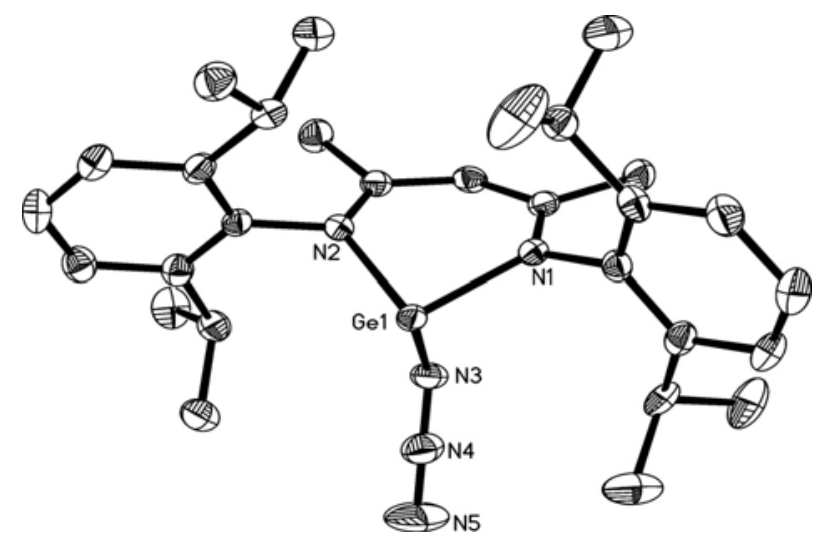

Figure 3.10. Molecular structure of 17 . Thermal ellipsoids are shown at $50 \%$ probability. $\mathrm{H}$ atoms are omitted for clarity reasons. Selected bond lengths $[\AA]$ and angles $\left[{ }^{\circ}\right]$ : Ge1-N3 2.002(3), N3-N4 1.198(4), N4-N5 1.149(4), Ge1-N1 1.980(2), Ge1-N2 1.973(2); Ge1-N3-N4 121.5(3), N3-N4-N5 176.3(4), N1-Ge1-N2 $90.69(11)$

Compound 17 crystallizes in the triclinic space group $P-1$ from $n$-hexane. There are three independent molecules in the unit cell (Figure 3.10). The IR spectrum of 17 exhibits a band at $2068 \mathrm{~cm}^{-1}$, which is assigned to the $\mathrm{N}_{3}$ stretching frequency. 
18 is a colorless solid soluble in benzene, THF, $n$-hexane, and $n$-pentane respectively and shows no decomposition on exposure to dry air. The ${ }^{1} \mathrm{H}$ NMR spectrum of $\mathbf{1 8}$ shows a singlet ( $\delta 0.01 \mathrm{ppm})$, which can be assigned to the $\mathrm{Si}\left(\mathrm{CH}_{3}\right)_{3}$ protons. Maintaining an $n$-hexane solution of 18 for two days at $-32{ }^{\circ} \mathrm{C}$ resulted in colorless single crystals suitable for X-ray structural analysis. 18 crystallizes in the monoclinic space group $P 2_{1} / n$, with one molecule of $\mathbf{1 8}$ in the asymmetric unit. 18 exists as a monomer in the solid state (Figure 3.11). There are no intermolecular hydrogen bonds observed in the crystal lattice. The coordination polyhedron around the germanium atom comprises four nitrogen atoms, two from the supporting ligand, and two others from the amide groups, featuring a distorted tetrahedral geometry. The four Ge-N bond lengths are almost similar. The Ge-N bond lengths of 5 and $\mathbf{1 7}$ and those of $\mathbf{1 8}$ are quite different due to the non-equal oxidation states of the germanium atoms, and it is very much closer to the Ge-N bond lengths in compound 16, due to the same oxidation state of the germanium atom.

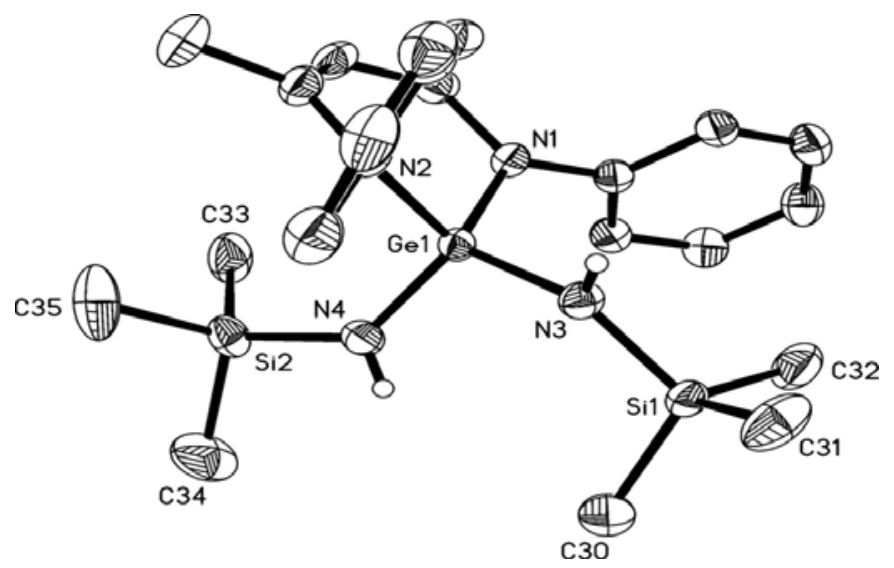

Figure 3.11. Molecular structure of 18. Thermal ellipsoids are shown at 50\% probability. H atoms except N3-H and N4-H and the iso-propyl groups are omitted for clarity reasons. Selected bond lengths $[\AA]$ and angles $\left[{ }^{\circ}\right]$ : Ge1-N1 1.8416(14), Ge1-N2 1.8413(15), Ge1-N3 1.8011(14), Ge1-N4 1.8224(15); N1-Ge1-N2 101.59(6), N1-Ge1-N3 112.77(7), N3-Ge1-N4 111.24(8), N2-Ge1-N4 111.54(7).

The formation of $\mathbf{1 8}$ requires the oxidation of germanium(II) to germanium(IV). Therefore the synthesis of 18 also involves an unprecedented oxidative addition-insertion with nitrene (:NSiMe 3 ), which is formed in situ from trimethylsilyl azide by elimination of dinitrogen, and insertion into the $\mathrm{Ge}(\mathrm{II})-\mathrm{H}$ bond, which leads to the formal conversion of the $\mathrm{GeH}$ hydride to a NH proton. Surprisingly there is no precedent reaction of group 14 known where nitrene is 
added and inserted simultaneously at the same element. We were not able to isolate any intermediate of this reaction. No evidence was found for any tautomeric equilibrium of $\mathbf{1 8}$.

\subsection{Reactions with Frustrated Lewis Carbene-Borane Pair}

The Lewis acid-base concept is fundamental for understanding numerous chemical reactions. ${ }^{[183]}$ This principle is essential for main group elements as well as for transition metals. ${ }^{[53]}$ In recent years, D. W. Stephan, ${ }^{[184]}$ B. Rieger, ${ }^{[185]}$ G. Erker, ${ }^{[186]}$ and M. Tamm ${ }^{[187]}$ were able to demonstrate that frustrated Lewis pairs of boranes, (in particular tris(pentafluorophenyl)borane, $\left.\mathrm{B}\left(\mathrm{C}_{6} \mathrm{~F}_{5}\right)_{3}\right),{ }^{[188]}$ in combination with sterically demanding phosphanes, amines, imines, and N-heterocyclic carbenes (NHCs), respectively, are excellent non-metallic systems for the activation of dihydrogen under mild conditions. NHCs and $\mathrm{B}\left(\mathrm{C}_{6} \mathrm{~F}_{5}\right)_{3}$ independently have also been employed for proton and hydride abstraction reactions. ${ }^{[145,189]}$ In compound $\mathbf{5}$, the hydrogen atoms in the backbone methyl group are protonic hydrogen and the hydrogen atom at the germanium center are hydridic hydrogen. Therefore, it seemed possible that $\mathrm{LGeH}(5)$ might be dehydrogenated with a combination of Lewis NHC borane pair. In the literature dehydrogenation of alkanes is reported using transition metal catalyst. ${ }^{[190]}$ Herein we report on the dehydrogenation of LGeH by a frustrated Lewis NHC borane pair under the formation of six-membered N-heterocyclic germylene $\mathrm{L}^{\prime} \mathrm{Ge},\left[\mathrm{L}^{\prime}=\mathrm{CH}\left\{\left(\mathrm{C}=\mathrm{CH}_{2}\right)(\mathrm{CMe})(\mathrm{NAr})_{2}\right\} ; \mathrm{Ar}=2,6-i \mathrm{Pr}_{2} \mathrm{C}_{6} \mathrm{H}_{3}\right] ;$ (19).

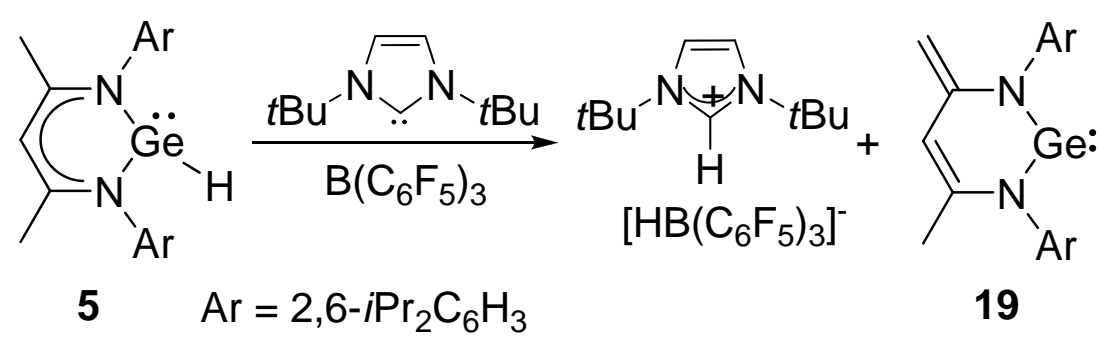

Scheme 3.10. Preparation of compoumd 19

A toluene solution of equimolar amounts of 5 and of $B\left(\mathrm{C}_{6} \mathrm{~F}_{5}\right)_{3}$ under stirring changed the color from red to colorless after a few minutes. To this reaction mixture we added an equivalent of 1,3-di-tert-butylimidazol-2-ylidene ${ }^{[191]}$ and the reaction mixture immediately turned to brown-red with the formation of an insoluble white precipitate (Scheme 3.10). The latter was identified as imidazolium borate salt $\left[(\mathrm{CHN} t \mathrm{Bu})_{2} \mathrm{CH}\right]\left[\mathrm{HB}\left(\mathrm{C}_{6} \mathrm{~F}_{5}\right)_{3}\right]^{[187]}$ and was 
recovered by filtration and dissolved in dichloromethane. The ${ }^{1} \mathrm{H}$ NMR spectrum indicates the formation of an imidazolium cation. The ${ }^{11} \mathrm{~B}$ NMR resonance of borate salt at $-25.3 \mathrm{ppm}$ is identical with a previously reported imidazolium borate salt. ${ }^{[187]}$ The soluble part of the reaction mixture was characterized to contain the heterocyclic germylene L'Ge (19), although there is no reaction between compound $\mathbf{5}$ and 1,3-di-tert-butylimidazol-2-ylidene. ${ }^{[191]}$ Compound 19 was previously reported by Driess et al. by the reaction of $\mathrm{LGeCl}$ with $\mathrm{LiN}\left(\mathrm{SiMe}_{3}\right)_{2} \cdot{ }^{[37]}$ 


\section{Chapter 4}

\section{Hydrostannylation Reaction of LSnH with Unsaturated Molecules}

In recent years the parent $\mathrm{SnH}_{2}$ has been prepared and characterised in an argon matrix. ${ }^{[84]} \mathrm{At}$ elevated temperature $\mathrm{SnH}_{2}$ changed to an insoluble solid of unknown structure. The terphenyl and $\beta$-diketiminate ligands have been used for the preparation of substituted tin(II) hydrides. The terphenyl derivatives show in the solid state dimeric structures, ${ }^{[80,82]}$ while the $\beta$ diketiminate moiety exhibits a terminal tin(II) hydride with very weak intermolecular interactions. ${ }^{[79]}$ Until recently the reactions of organotin hydrides were based on tin(IV) precursor. Di- and triorganotin hydrides of composition $\mathrm{R}_{2} \mathrm{SnH}_{2}$ and $\mathrm{R}_{3} \mathrm{SnH}$ with formal oxidation state of $\mathrm{Sn}(\mathrm{IV})$ show a rich variety of chemical transformations. ${ }^{[192]}$ The preferred reagent of this class of compounds is the tributyltin hydride, and it is widely used as a reducing agent in organic and inorganic chemistry. ${ }^{[193]}$ To the best of our knowledge there are no hydrostannylation reactions described using tin(II) hydride. Herein, we report the first hydrostannylation reactions of carbon dioxide, aldehydes, ketones, alkines, and carbodiimides with LSnH (20).

\subsection{Stannylene Hydride Starting Material}

Initially our group reported the synthesis of $\mathrm{LSnH}(\mathbf{2 0})$ from the reaction of the corresponding $\mathrm{LSnCl},{ }^{[146]}$ with one equivalent of $\mathrm{AlH}_{3} \cdot \mathrm{NMe}_{3}{ }^{[155]}$ in toluene, just like the preparation of LGeH. ${ }^{[79]}$ Preparation of $\mathbf{2 0}$ from $\mathrm{AlH}_{3} \cdot \mathrm{NMe}_{3}$ resulted in the formation of the expected product contaminated with small amounts of the starting material LSnCl. This slightly impure LSnH leads to products containing chlorine, when it was used in hydrostannylation reactions. Furthermore this LSnH is unstable when contaminated with chlorine and decomposes in an inert glove box within four days. Therefore the method of preparation had to be changed. Treatment of $\mathrm{LSnCl}$ with potassium trisecbutylborohydride, $\left(\mathrm{K}\left[\mathrm{HB}(\sec \mathrm{Bu})_{3}\right]\right)$, in toluene at $10{ }^{\circ} \mathrm{C}$ afforded the tin(II) hydride LSnH (20) in high yield (Scheme 4.1).

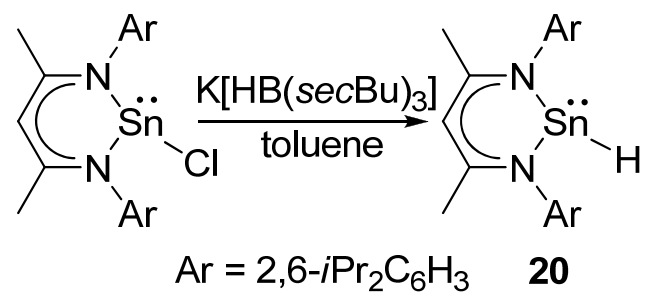

Scheme 4.1. Preparation of compound 20 
The crude reaction product was recrystallized from $n$-hexane and obtained as pure yellow crystals which were characterized by ${ }^{1} \mathrm{H}(\delta 13.96 \mathrm{ppm})$ and ${ }^{119} \mathrm{Sn}\left(\delta-4.45 \mathrm{ppm},{ }^{1} J\left({ }^{119} \mathrm{Sn},{ }^{1} \mathrm{H}\right)=\right.$ $64 \mathrm{~Hz}$ ) NMR spectroscopy. The chemical shift for ${ }^{119} \mathrm{Sn}$ of 20 given in ref. [79] is that of LSnCl.

\subsection{Reaction with Carbon Dioxide}

Carbon dioxide is a readily accessible atmospheric gas that could be a useful feedstock for organic compounds. ${ }^{[194]}$ The kinetic and thermodynamic stability of carbon dioxide present significant challenges in designing efficient chemical transformations. Herein, we report on the synthesis of a stannylene formate $\mathrm{LSn}-\mathrm{O}-\mathrm{C}(\mathrm{O}) \mathrm{H}(\mathbf{2 1})$, by the reaction of $\mathbf{2 0}$ with carbon dioxide at room temperature without any catalyst in quantitative yield (Scheme 4.2).

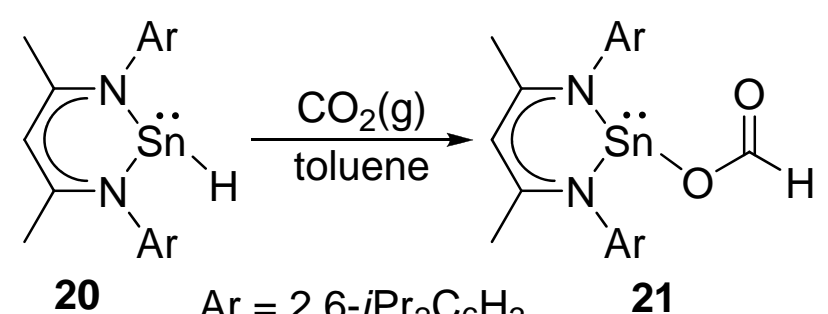

Scheme 4.2. Preparation of compound 21

21 is a colorless solid, soluble in benzene, THF, $n$-hexane, and $n$-pentane and shows no decomposition on exposure to air. 21 was characterized by multinuclear NMR and IR spectroscopy, EI mass spectrometry, elemental analysis, and X-ray structural analysis. The ${ }^{1} \mathrm{H}$ NMR spectrum of 21 exhibits a singlet ( $\left.\delta 8.97 \mathrm{ppm}\right)$ which can be assigned to the $\mathrm{CH}$ proton and is flanked by $\mathrm{Sn}$ satellite lines $\left({ }^{3} J\left({ }^{119} \mathrm{Sn},{ }^{1} \mathrm{H}\right)=52 \mathrm{~Hz}\right)$. The ${ }^{119} \mathrm{Sn}$ NMR resonance of 21 arises at $\delta-360 \mathrm{ppm}$, which is very much different from the starting compound $\mathbf{2 0}$. The IR spectrum shows two bands at 2700 and $1641 \mathrm{~cm}^{-1}$, which are assigned to the $\mathrm{C}-\mathrm{H}$ and $\mathrm{C}=\mathrm{O}$ stretching frequencies.

Colorless compound 21 crystallizes in the triclinic space group $P-1$, with two monomers in the asymmetric unit from saturated $n$-hexane solution at $-32{ }^{\circ} \mathrm{C}$ after two days. The coordination around the tin atom features a distorted tetrahedral geometry with one lone pair (Figure 4.1). 


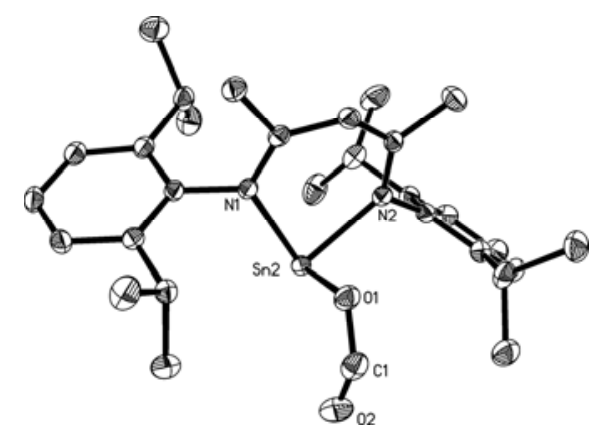

Figure 4.1. Molecular structure of 21. Thermal elipsoids are shown at 50\% probability. $\mathrm{H}$ atoms are omitted for clarity reasons. Selected bond lengths $[\AA]$ and angles $\left[^{\circ}\right]$ : Sn2-O1 2.1353(15), O1-C1 1.299(2), C1-O2 1.209(3), Sn2-N1 2.1655(17), Sn2-N2 2.1752(15); Sn2-O1-C1 116.40(13), O1-C1-O2 126.78(18), N1-Sn2N1 86.42(6).

\subsection{Reactions with Carbonyl Compounds}

The carbonyl group and its transformation to other functional groups is very important in organic chemistry. ${ }^{[195]}$ In literature there are numerous reports on hydrostannation reaction of compounds with carbonyl groups using tin(IV) hydride, ${ }^{[196]}$ and carbonyl group insertion into the tin hydrogen bond of various complexes is well established. ${ }^{[197]}$ Herein we demonstrate for the first time the hydrostannylation of a carbonyl group using tin(II) hydride. Examples are the reaction of ferrocene carbaldehyde, benzophenone, 2-benzoyl pyridine, di(2pyridyl)ketone, and dicyclopropylketone with $\mathrm{LSnH}(\mathbf{2 0})$. Treatment of 20 with ferrocene carbaldehyde, benzophenone, 2-benzoyl pyridine, di(2-pyridyl)ketone, and dicyclopropylketone leads quantitatively to the stannylene alkoxides 22-26, respectively with a $\mathrm{Sn}(\mathrm{II})-\mathrm{O}-\mathrm{CH}$ framework that is formed by nucleophilic hydride addition to the respective carbon of the carbonyl group (Scheme 4.3).

Compounds 22-26 were monitored by ${ }^{1} \mathrm{H}$ NMR spectra. Sharp resonances in the ${ }^{1} \mathrm{H}$ NMR of 22-26 gave the initial indication that the products have been formed in high yield. Ferrocene moieties are important in organometallic chemistry, with respect to electrochemistry ${ }^{[198]}$ and material science, because bridging ferrocenophanes undergo polymerization and generate organometallic polymers with high molecular weight. ${ }^{[199]}$ The reaction of $\mathbf{2 0}$ with ferrocene carbaldehyde generates bimetallic tin(II) alkoxide 22 (Scheme 4.3). 


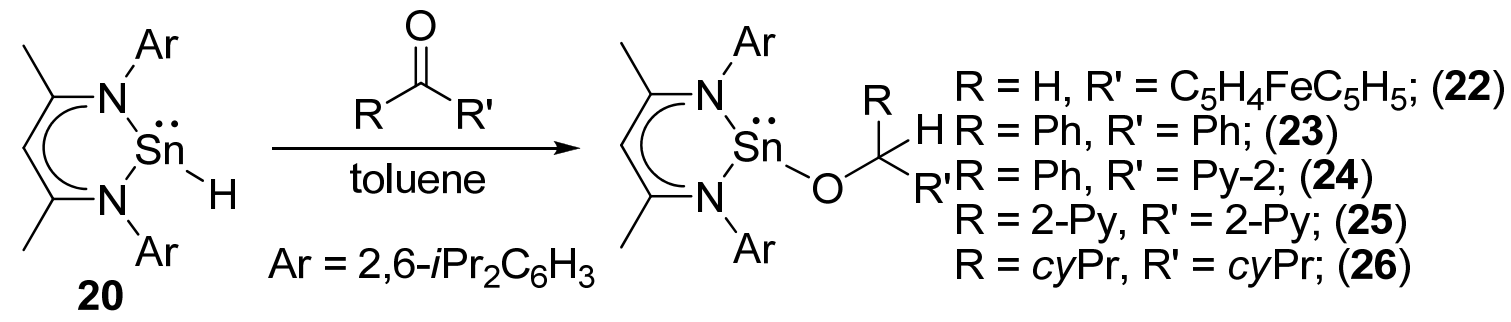

Scheme 4.3. Preparation of compounds $22,23,24,25$, and 26

22 is an orange solid, soluble in benzene, THF, $n$-hexane, and $n$-pentane and shows no decomposition on exposure to air. 22 was characterized by multinuclear NMR and IR spectroscopy, EI mass spectrometry, elemental analysis, and X-ray structural analysis (Figure 4.2). The ${ }^{1} \mathrm{H}$ NMR spectrum of 22 exhibits a singlet ( $\left.\delta 4.81 \mathrm{ppm}\right)$, which can be assigned to the $\mathrm{CH}_{2}$ protons and is flanked by Sn satellite lines $\left({ }^{3} \mathrm{~J}\left({ }^{119} \mathrm{Sn},{ }^{1} \mathrm{H}\right)=13.5 \mathrm{~Hz}\right)$. The ${ }^{119} \mathrm{Sn} \mathrm{NMR}$ resonance of 22 arises at $\delta-262 \mathrm{ppm}$.

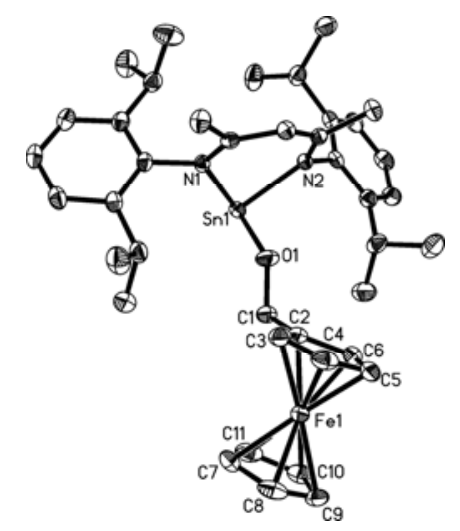

Figure 4.2. Molecular structure of 22. Thermal elipsoids are shown at $50 \%$ probability. $\mathrm{H}$ atoms are omitted for clarity reasons. Selected bond lengths $[\AA]$ and angles $\left[{ }^{\circ}\right]$ : Sn1-O1 2.0253(13), O1-C1 1.411(2), Sn1-N1 2.1834(14), Sn1-N2 2.1894(14); Sn1-O1-C1 129.13(11), N1-Sn1-O1 87.89(6), N1-Sn1-N2 85.10(5).

23, 24, and 25 were characterized by multinuclear NMR spectroscopy, EI mass spectrometry, elemental analysis. Furthermore compound $\mathbf{2 4}$ was characterised by X-ray structural analysis (Figure 4.3). The ${ }^{1} \mathrm{H}$ NMR spectra of 23, 24, and 25 exhibit a singlet ( $\delta 5.97,6.28$, and 6.59 ppm respectively) which can be assigned to the quaternary $\mathrm{CH}$ proton, and the complete disappearance of the proton resonance of $\mathrm{Sn}-H$ was observed. This indicates the conversion of the tin(II)-hydride to the corresponding tin(II)-alkoxide. The ${ }^{119} \mathrm{Sn} \mathrm{NMR}$ exhibits resonances at $-256,-286$, and $-324 \mathrm{ppm}$ for compounds $\mathbf{5}, \mathbf{6}$, and $\mathbf{7}$. The three values are different due to the non identical electronic nature of the phenyl rings when compared with 
those of the 2-pyridyl rings. In the EI mass spectra the molecular ion peaks were observed at $m / z 719,720$, and 722 as the base peaks for $\mathbf{2 3 , 2 4}$, and 25.

24 crystallizes in the triclinic space group $P-1$ with one monomer in the asymmetric unit (Figure 4.3). The Sn-O bond lengths $(2.0414(15) \AA$ ) are comparable with the Sn-O bond

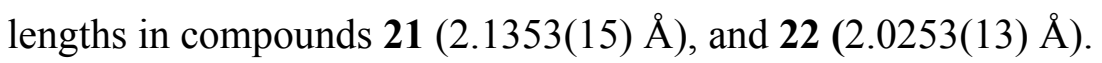

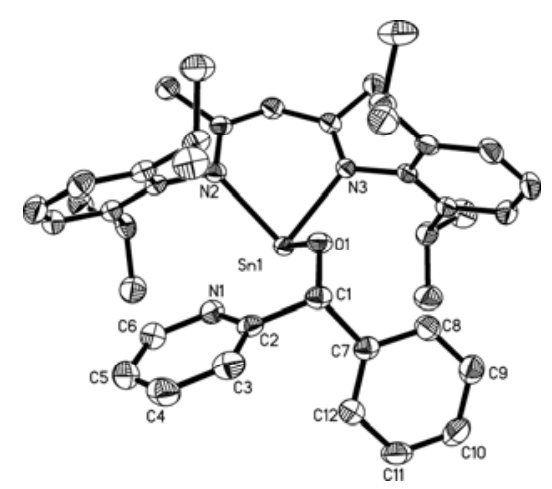

Figure 4.3. Molecular structure of 24. Thermal elipsoids are shown at $50 \%$ probability. $\mathrm{H}$ atoms are omitted for clarity reasons. Selected bond lengths $[\AA]$ and angles $\left[{ }^{\circ}\right]$ : Sn1-O1 2.0414(15), Sn1-N2 2.2420(16), Sn1-N3 2.2632(18); N2-Sn1-O1 99.99(6), N2-Sn1-N3 82.10(6).

At room temperature there is no reaction observed, when $\mathrm{LSnH}$ is treated with dicyclopropylketone in toluene. However after refluxing this mixture for $12 \mathrm{~h}$ compound 26 is formed in high yield. Compound 26 is composed of two cyclopropyl rings and to our surprise they were not decomposed during the reaction. The ${ }^{1} \mathrm{H}$ NMR spectrum of $\mathbf{1 0}$ exhibits a triplet $(\delta 2.47 \mathrm{ppm})$, which can be assigned to the quaternary $\mathrm{CH}$ proton which is coupled by two $\mathrm{C}-\mathrm{H}$ protons from the two cyclopropyl rings with a coupling constant of $7.3 \mathrm{~Hz}$. In the ${ }^{1} \mathrm{H}$ NMR spectrum of compound 26 the quaternary proton $(\delta 2.47 \mathrm{ppm})$ resonates upfield compared to the corresponding proton in compounds $\mathbf{2 3 , 2 4}$, and 25 ( $\delta$ 5.97, 6.28, and 6.59 ppm respectively). This is mainly due to the different electronic nature of the two cyclopropyl rings in compound 26. The other protons of the cyclopropyl rings show the expected resonances. The two quaternary $\mathrm{CH}$ protons of the two cyclopropyl rings exhibit a multiplet each at $0.75 \mathrm{ppm}$. The four $\mathrm{CH}_{2}$ groups of the two cyclopropyl rings are not identical; they show four multiplets $(\delta 0.21,0.02,-0.07,-0.25 \mathrm{ppm})$. Each multiplet is not consistent with each methylene group. It has been found that each multiplet corresponds to one proton from each of the two cyclopropyl rings. 
Single crystal of $\mathbf{2 6}$ suitable for X-ray structural analysis was obtained from $n$-hexane solutions. 26 crystallizes in the triclinic space group $P-1$. The molecular structure of $\mathbf{2 6}$ is shown in Figure 4.4. The asymmetric unit of $\mathbf{2 6}$ contains one formula unit of the compound, and two of the molecules in each unit cell. As predicted, based on the ${ }^{1} \mathrm{H}$ NMR spectrum and EI mass spectrum, compounds 26 contains a $\mathrm{Sn}(\mathrm{II})-\mathrm{O}-\mathrm{CH}$ core with a two cyclopropyl groups.

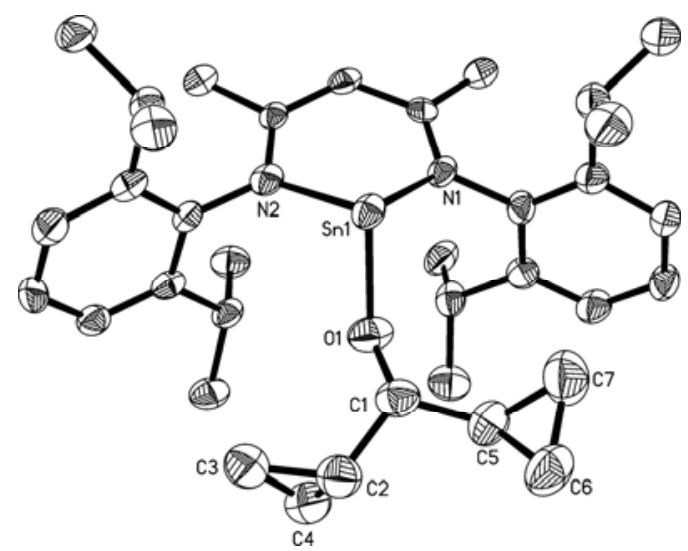

Figure 4.4. Molecular structure of 26. Selected bond lengths $[\AA]$ and angles $\left[^{\circ}\right]$; anisotropic displacement parameters are depicted at the $50 \%$ probability level and all restrained refined hydrogen atoms are omitted for clarity: Sn1-O1 2.024(3), Sn1-N1 2.199(4), O1-C1 1.431(6); N1-Sn1-N2 82.79(14), N1-Sn1-O1 95.80(13), Sn1-O1-C1 117.5(3).

\subsection{Reactions with Compounds Containing $C \equiv C$ Triple Bonds}

The hydrostannation of olefins and alkines is well known since nearly 50 years and follows a polar or a free radical pathway depending on substituents and conditions. ${ }^{[200]}$ In contrast to this result the present hydrostannylation reaction of alkines with $\mathrm{LSnH}(\mathbf{2 0})$ proceeds without any catalyst although alkine insertion into transition metal hydride bonds is well established. ${ }^{[201]} \mathrm{LSnH}$ (20) reacts with the alkines $\mathrm{HC} \equiv \mathrm{CCO}_{2} \mathrm{Me}, \mathrm{HC} \equiv \mathrm{CCO}_{2} \mathrm{Et}$, $\mathrm{MeC} \equiv \mathrm{CCO}_{2} \mathrm{Et}, \mathrm{MeO}_{2} \mathrm{CC} \equiv \mathrm{CCO}_{2} \mathrm{Me}, \mathrm{EtO}_{2} \mathrm{CC} \equiv \mathrm{CCO}_{2} \mathrm{Et}$, and $t \mathrm{BuO}_{2} \mathrm{CC} \equiv \mathrm{CCO}_{2} t \mathrm{Bu}$ at room temperature to form tin(II) substituted alkenes 27, 28, 29, 30, 31, and 32. Compound 20 reacts with $\mathrm{HC} \equiv \mathrm{CCO}_{2} \mathrm{Me}$ and $\mathrm{HC} \equiv \mathrm{CCO}_{2}$ Et respectively at room temperature to form the vinyl stannylenes 27 and $\mathbf{2 8}$ (Scheme 4.4). 


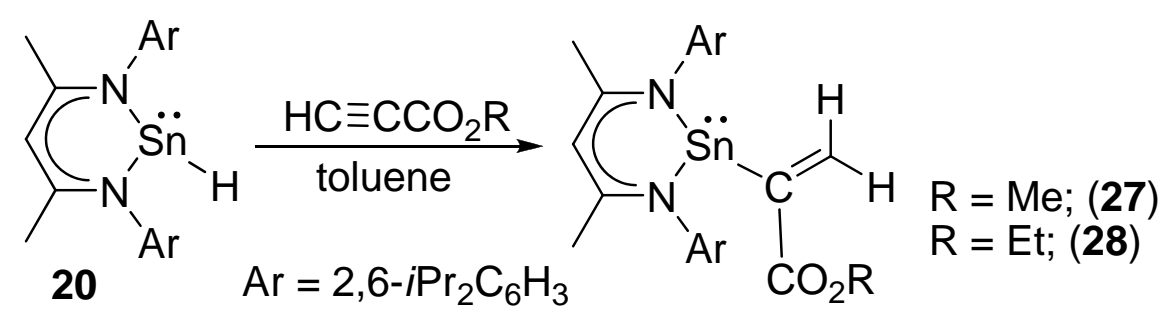

Scheme 4.4. Preparation of compounds 27 and 28

27 and 28 are obtained by the 1,2-addition of stannylene hydride LSnH to the terminal alkines and the result is the transfer of the hydrogen atom and stannylene to the carbon carbon triple bond (Scheme 4.4), rather than the elimination of $\mathrm{H}_{2}$ due to the different polarized $\mathrm{Sn}-\mathrm{H}$ and $\mathrm{C}-\mathrm{H}$ bonds.

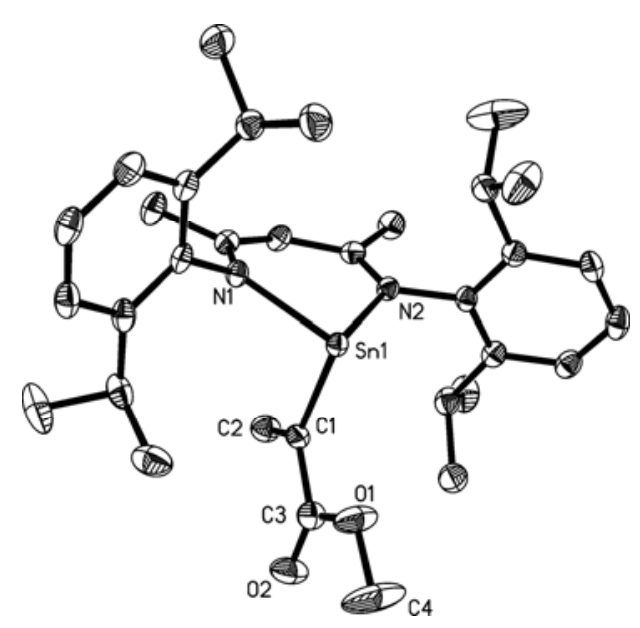

Figure 4.5. Molecular structure of 27. Selected bond lengths $[\AA]$ and angles $\left[^{\circ}\right]$; anisotropic displacement parameters are depicted at the $50 \%$ probability level and all restrained refined hydrogen atoms are omitted for clarity: Sn1-C1 2.241(2), Sn1-N1 2.202(2), C1-C3 1.488(3); N1-Sn1-N2 85.64(7), N1-Sn1-C1 93.06(8), Sn1-C1-C3 116.79(17).

The ${ }^{1} \mathrm{H}$ NMR spectrum of 28 exhibits two broad resonances ( $\delta 6.25$ and $\left.5.85 \mathrm{ppm}\right)$ which correspond to the two alkenyl protons. Moreover, the ${ }^{1} \mathrm{H}$ NMR spectrum shows a quartet and a triplet resonance ( $\delta 4.07$ and $0.87 \mathrm{ppm})$ corresponding to the two different types of $\mathrm{CH}$ protons of the ethyl moieties. Compound 27 crystallizes in the triclinic space group $P-1$, with one monomer in the asymmetric unit, and with two molecules in each unit cell. Single crystals were obtained from a saturated $n$-hexane solution at $-32{ }^{\circ} \mathrm{C}$ after two days. The coordination polyhedron around the tin atom features a distorted tetrahedral geometry with a stereochemically active lone pair (Figure 4.5). 
Furthermore we were interested in the selectivity of the addition across the carbon carbon triple bond. Therefore we selected the disubstituted alkine, $\mathrm{MeC} \equiv \mathrm{CCO}_{2}$ Et. Compound 20 reacts with $\mathrm{MeC} \equiv \mathrm{CCO}_{2} \mathrm{Et}$ at room temperature to form stannylene 29 (Scheme 4.5). The ${ }^{1} \mathrm{H}$ NMR spectrum of 29 shows a broad resonance $(\delta 6.64 \mathrm{ppm})$ which can be tentatively assigned to the vinyl proton. The IR spectrum of this compound exhibits a strong band at $1686 \mathrm{~cm}^{-1}$, which can be tentatively assigned to the carbonyl group.

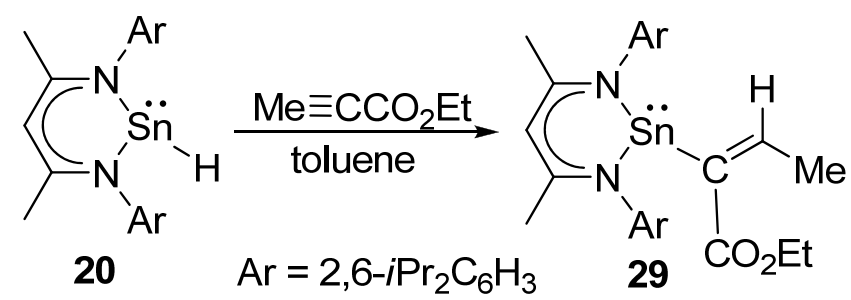

Scheme 4.5. Preparation of compound 29

The crystal structure (Figure 4.6) of $\mathbf{2 9}$ reveals a cis orientation of the tin and hydrogen atom across the double bond and crystallizes in the triclinic space group $P-1$. Also in solution there is only one isomer observed. Therefore $\mathbf{2 9}$ is obtained by 1,2-syn-addition of $\mathbf{2 0}$ to the alkine and the result is the transfer of the hydrogen atom and stannylene across the carbon carbon triple bond.

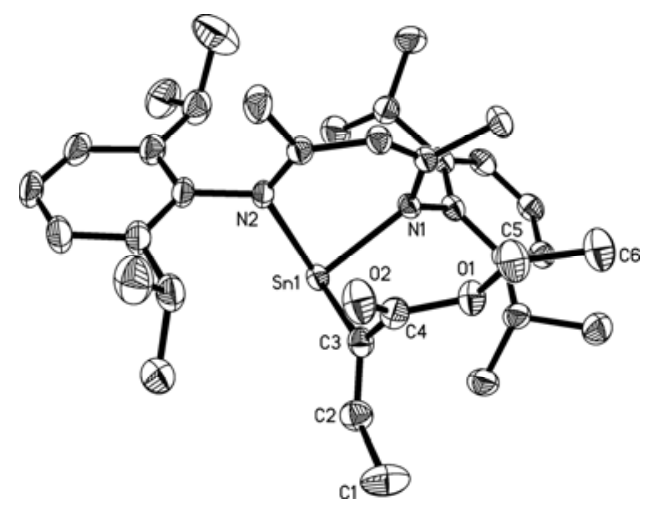

Figure 4.6. Molecular structure of 29. Thermal elipsoids are shown at 50\% probability. $\mathrm{H}$ atoms are omitted for clarity reasons.Selected bond lengths $[\AA \AA]$ and angles $\left[{ }^{\circ}\right]$ : Sn1-C3 2.242(3), C3-C2 1.335(4), C4-O2 1.2101(3), Sn1-N1 2.1990(19), Sn1-N2 2.199(2); Sn1-C3-C2 114.13(19), Sn1-C3-C4 127.37(13), N2-Sn1-N2 86.31(8). 
Moreover we selected the symmetric disubstituted alkines, dimethyl acetylenedicarboxylate, diethyl acetylenedicarboxylate, and ditertiarybutyl acetylenedicarboxylate. $\mathbf{2 0}$ reacts with $\mathrm{RO}_{2} \mathrm{CC} \equiv \mathrm{CCO}_{2} \mathrm{R}(\mathrm{R}=\mathrm{Me}$, Et, and $t \mathrm{Bu})$ in toluene at room temperature to form the two isomers with $E$ - and $Z$ - stannylene substituted alkene in a different ratio (1:0.7 for 30, 1.00:0.69 for 31, and 1.00:1.52 for 32) (Scheme 4.6). Each ${ }^{1} \mathrm{H}$ NMR spectrum of 30, 31, and 32 exhibits two singlets which are arranged between the tin satellites with two different coupling constants. The ${ }^{119} \mathrm{Sn}$ NMR resonances arises at $\delta-132$ and $-211 \mathrm{ppm}$, for $E$ - and $Z$ isomers of 30, $\delta$-130 and $-211 \mathrm{ppm}$, for $E$ - and $Z$ - isomers of 31, and the resonance at $\delta$-123 and -205 ppm for $E$ - and $Z$ - isomers of $\mathbf{3 2}$.

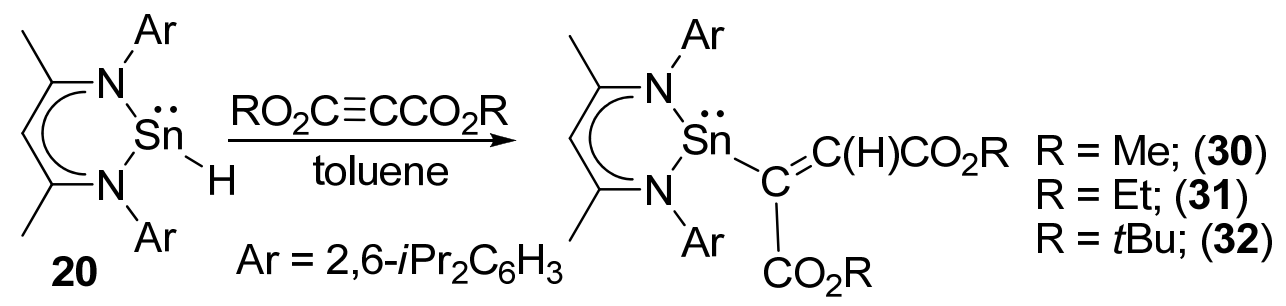

Scheme 4.6. Preparation of compounds 30, 31, and 32

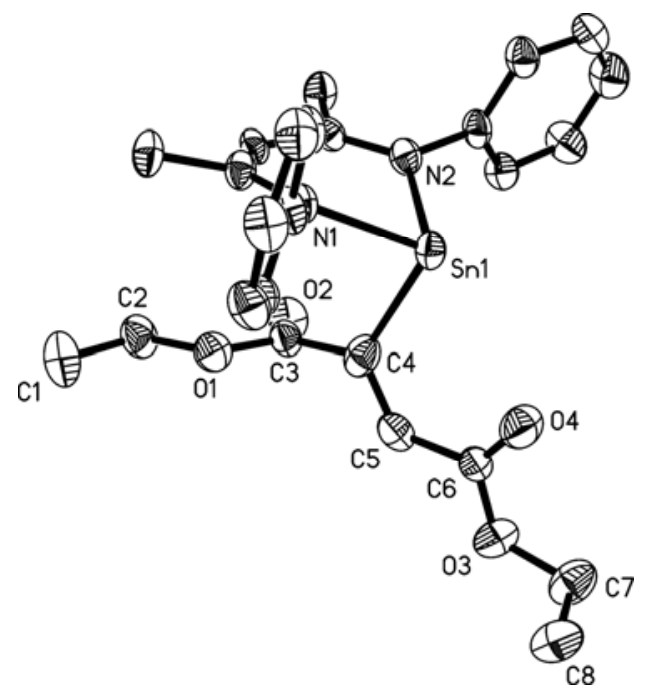

Figure 4.7. Molecular structure of 31. Selected bond lengths $[\AA]$ and angles $\left[{ }^{\circ}\right]$; anisotropic displacement parameters are depicted at the $50 \%$ probability level and all restrained refined hydrogen atoms and iso-propyl groups are omitted for clarity: Sn1-N1 2.207(5), Sn1-C4 2.330(8), C4-C5 1.331(9); N1-Sn1-N2 85.19(17), N1-Sn1-C4 92.3(2), Sn1-C4-C5 121.5(5).

31 crystallizes in the monoclinic space group $P 2_{1} / c$, with one monomer in the asymmetric unit from saturated $n$-hexane solution at $-32{ }^{\circ} \mathrm{C}$ after one day (Figure 4.7). In the crystalline state we were only able to isolate the $Z$ - isomer. 


\subsection{Reactions with Compounds Containing $C=N$ Double Bond}

The reaction of $\mathbf{2 0}$ with compounds containing $\mathrm{C}-\mathrm{N}$ multiple bonds was performed with dicyclohexyl carbodiimide (DCC), and diisopropyl carbodiimide $(\mathrm{RN}=\mathrm{C}=\mathrm{NR}, \mathrm{R}=i \operatorname{Pr}$ and $\mathrm{Cy}$ ). This reaction proceeds rapidly and quantitatively at room temperature to give the spirocyclic compounds $\mathbf{3 3}$ and $\mathbf{3 4}$ containing the four- and six-membered heterocycles of composition $\mathrm{C}_{3} \mathrm{~N}_{2} \mathrm{Sn}$ and $\mathrm{CN}_{2} \mathrm{Sn}$ (Scheme 4.7).

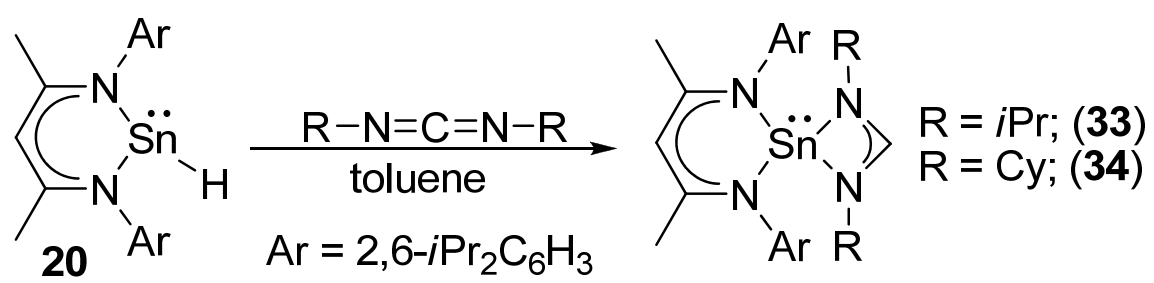

Scheme 4.7. Preparation of compounds 33 and $\mathbf{3 4}$

Compoounds 33 and $\mathbf{3 4}$ were characterised by multinuclear NMR spectroscopy, EI mass spectroscopy, and elemental analysis. In the ${ }^{1} \mathrm{H}$ NMR spectra of $\mathbf{3 3}$ and $\mathbf{3 4}$ the $\mathrm{C}-H$ proton of the four-membered ring shows a singlet ( $\delta 8.26$ and $8.30 \mathrm{ppm})$ and other resonances as expected while the ${ }^{119} \mathrm{Sn}$ NMR resonance arises at $\delta-177.69$ and $-175 \mathrm{ppm}$ respectively for 33 and 34. In the EI mass spectra the molecular ion peaks were observed at $m / z 664$ and 744 as the base peaks for $\mathbf{3 3}$ and $\mathbf{3 4}$ respectively.

\subsection{Reaction of LSnH with Fluorinated Carbonyl Compounds: Nucleophilic Addition Reaction vs Hydrogen for Fluorine Exchange in C-F Bonds}

Fluorine's peculiar characteristics such as high electronegativity, low polarizability, and small covalent radius render fluorocarbons thermally stable, water repellent, and resistant to chemical degradation. ${ }^{[202]}$ These unique properties, along with the great strength of the C-F bond (120-129 kcal/mol for aliphatic and olefinic C-F bonds, and up to $154 \mathrm{kcal} / \mathrm{mol}$ for $\left.\mathrm{C}_{6} \mathrm{~F}_{6}\right),{ }^{[203]}$ make fluorocarbons valuable refrigerants, pesticides, and nonadhesive polymers but also very environmentally persistent. Therefore, methods for chemically manipulating fluorocarbons are sought to either degrade or add value to these materials. The hydrogen for fluorine exchange in fluorocarbons is a thermodynamically favorable reaction that does not occur in absence of a catalyst. ${ }^{[204]}$ For example, passing a mixture of $\mathrm{C}_{6} \mathrm{~F}_{6}$ and dihydrogen 
over $\mathrm{Pd} / \mathrm{C}$ or $\mathrm{Pt} / \mathrm{C}$ at $300{ }^{\circ} \mathrm{C}$ gives a mixture of aromatic hydrofluorocarbons; $\mathrm{Pt} / \mathrm{C}$ at $58 \%$ conversion gives $\mathrm{C}_{6} \mathrm{~F}_{5} \mathrm{H}$ and mixed isomers of $\mathrm{C}_{6} \mathrm{~F}_{4} \mathrm{H}_{2}$ and $\mathrm{C}_{6} \mathrm{~F}_{3} \mathrm{H}_{3}$ in a 4:3:1 ratio. ${ }^{[205]}$

We have already discussed the nucleophilic addition reaction of $\mathrm{LGeH}(\mathbf{5})$ with compounds containing trifluoroacetyl group. There were no indications that the aliphatic C-F bonds can exchange with the Ge-H bond. We present here the reaction of $\mathrm{LSnH}$ (20) with fluorine substituted carbonyl compounds namely 2,2,2-trifluoro acetophenone, 2,2,2-trifluoro acetothiophane, pentafluoro benzophenone, perfluoro benzophenone, 4-fluoro benzophenone, and pentafluoro benzaldehyde.<smiles></smiles>

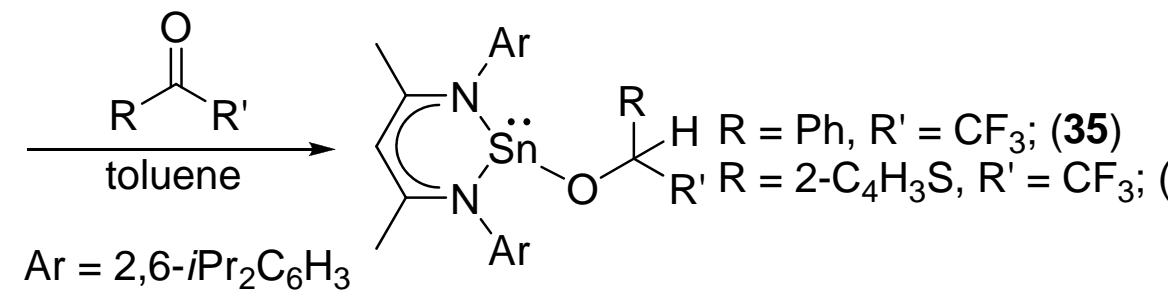

Scheme 4.8. Preparation of compounds 35 and $\mathbf{3 6}$

The reaction of $\mathrm{LSnH}$ with 2,2,2-trifluoro acetophenone and 2,2,2-trifluoro acetothiophane leads to the tin(II) alkoxides 35 and 36 (Scheme 4.8). There are no indications that the C-F bonds of the $\mathrm{CF}_{3}$ group are affected by the $\mathrm{LSnH}$. Compounds 35 and $\mathbf{3 6}$ have one $\mathrm{CF}_{3}$ group and display an interesting NMR spectrum.

${ }^{1} \mathrm{H}$ NMR spectra of $\mathbf{3 5}$ and $\mathbf{3 6}$ exhibit a quartet ( $\delta 5.15$ and $5.45 \mathrm{ppm}$ respectively) which corresponds to the quaternary $\mathrm{CH}$ proton. The ${ }^{19} \mathrm{~F}$ NMR resonance arises as a doublet $(\delta$ 76.29 and -77.38 ppm respectively) with a coupling constant of 6 and $7 \mathrm{~Hz}$ and is flanked by Sn satellite lines $\left({ }^{4} J\left({ }^{119} \mathrm{Sn},{ }^{1} \mathrm{H}\right)=42\right.$ and $\left.56 \mathrm{~Hz}\right)$. The four isopropyl groups of $\mathbf{3 5}$ and $\mathbf{3 6}$ are showing four different resonances and even the two methyl groups in the backbone exhibit two different signals in the proton NMR spectra. 


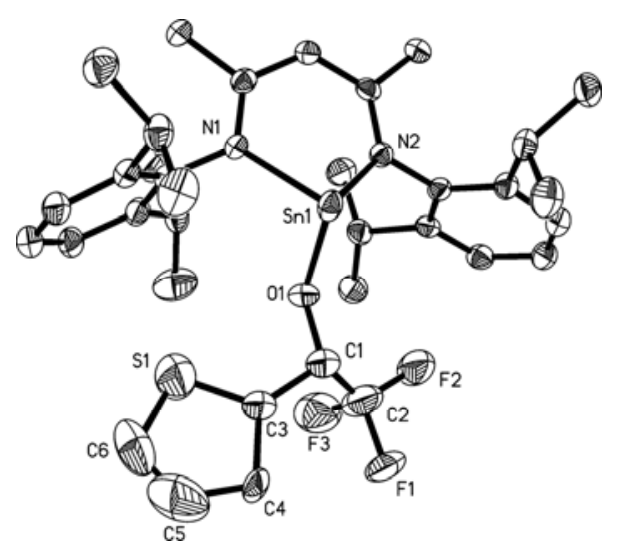

Figure 4.8. Molecular structure of 36. Selected bond lengths $[\AA]$ and angles $\left[^{\circ}\right]$; anisotropic displacement parameters are depicted at the $50 \%$ probability level and all restrained refined hydrogen atoms are omitted for clarity: Sn1-O1 2.046(3), Sn1-N1 2.194(3), O1-C1 1.418(6); N1-Sn1-N2 83.30(11), N1-Sn1-O1 93.04(12), Sn1-O1-C1 114.5(4).

Single crystal of $\mathbf{3 6}$ suitable for X-ray structural analysis was obtained from $n$-hexane solution. 36 crystallizes in the triclinic space group $P-1$. The molecular structure of $\mathbf{3 6}$ is shown in Figure 4.8. The asymmetric unit of $\mathbf{3 6}$ contains one formula of the compound, and two of the molecules in each unit cell. As predicted, based on the ${ }^{1} \mathrm{H}$ NMR spectrum and EI mass spectrum, compound 36 contains a $\mathrm{Sn}(\mathrm{II})-\mathrm{O}-\mathrm{CH}$ core with a $\mathrm{CF}_{3}$ group. The three coordinate tin atom is surrounded by two $\mathrm{N}$ atoms of the $\beta$-diketiminato ligand, and an exocyclic $\mathrm{O}$ atom.

When LSnH is treated separately with equivalent amounts of pentafluoro benzophenone $\left(\mathrm{PhCOC}_{6} \mathrm{~F}_{5}\right)$ and perfluoro benzophenone $\left(\mathrm{C}_{6} \mathrm{~F}_{5} \mathrm{COC}_{6} \mathrm{~F}_{5}\right)$ in toluene at room temperature, the formation of two types of products occurs (Scheme 4.9). This is clearly shown from ${ }^{119} \mathrm{Sn}$ NMR spectra. One is the $\operatorname{LSnF}(\mathbf{1})$ and another one is the nucleophilic addition product tin(II) alkoxide $\mathrm{LSnOCH}\left(\mathrm{C}_{6} \mathrm{~F}_{5}\right) \mathrm{Ph}(\mathbf{3 7})$, and $\mathrm{LSnOCH}\left(\mathrm{C}_{6} \mathrm{~F}_{5}\right)_{2}(\mathbf{3 8})$ respectively.<smiles>CC1=CC(C)N([Al])[Sn+](I)N1[Al]</smiles>

20<smiles>[R]C(=O)C1CCCC1</smiles>

$\mathrm{Ar}=2,6-i \mathrm{Pr}_{2} \mathrm{C}_{6} \mathrm{H}_{3}$<smiles>[R]C([R])O[Sn+]1C(C)C=C(C)N([Al-])[N+]1[Al-]</smiles>

$\mathrm{R}=\mathrm{Ph}, \mathrm{R}^{\prime}=\mathrm{C}_{6} \mathrm{~F}_{5} ;(37)$ $\mathrm{R}=\mathrm{C}_{5} \mathrm{~F}_{5}, \mathrm{R}^{\prime}=\mathrm{C}_{6} \mathrm{~F}_{5} ;(38)$<smiles>[R]C(=O)c1c(F)c(F)cc(F)c1F</smiles>

$\mathrm{R}=\mathrm{Ph}$ and $\mathrm{C}_{6} \mathrm{~F}_{5}$

Scheme 4.9. Preparation of compounds 37 and 38 
There are no indications that in the alkoxide products containing fluorine exchange occurred at the aromatic group in either case. In the ${ }^{1} \mathrm{H}$ NMR spectrum the quaternary proton from the LSn-O-CH moiety is showing the singlet at $\delta 6.41$ and $6.72 \mathrm{ppm}$ respectively, which is comparable with those of analogous compounds. In the EI mass spectrum of $\mathbf{3 8}$ there is observed the molecular ion peak at $m / z 900$ as the base peak. The formation of LSnF (1) and the alkoxide product ratio are calculated by the integration of the resonances in the ${ }^{1} \mathrm{H}$ NMR spectrum. In the case of the reaction between LSnH and pentafluoro benzophenone, the product ratio is $0.6: 1.0$, and the ${ }^{119} \mathrm{Sn}$ NMR resonance arises at $\delta-217 \mathrm{ppm}$ for compound $\mathrm{LSnOCH}\left(\mathrm{C}_{6} \mathrm{~F}_{5}\right) \mathrm{Ph}(37)$, and the other one is the doublet for $\operatorname{LSnF}\left(\delta-363,-380 \mathrm{ppm},{ }^{2} J(\mathrm{Sn}-\right.$ F) $=3100 \mathrm{~Hz}$ ). The latter signal matches well with that of LSnF. For the reaction of LSnH and perfluoro benzophenone, the product ratio is 0.8:1.0, and the ${ }^{119} \mathrm{Sn}$ NMR resonance arises at $\delta$-252 ppm for $\operatorname{LSnOCH}\left(\mathrm{C}_{6} \mathrm{~F}_{5}\right)_{2}(\mathbf{3 8})$. The ${ }^{1} \mathrm{H}$ NMR spectrum of compound 38 shows a singlet $(\delta 4.77 \mathrm{ppm})$ for the $\gamma-\mathrm{CH}$ protons and two septets ( $\delta 3.46$ and $3.02 \mathrm{ppm}$ ) corresponding to the two different types of $\mathrm{CH}$ protons of the $i \mathrm{Pr}$ moieties. The other signals are as expected. The fate of the pentafluoro benzophenone and perfluoro benzophenone after fluorine exchange are products containing symmetric substituted fluorine atoms, due to para fluorine exchange. In ${ }^{1} \mathrm{H}$ NMR spectrum is present a triplet of triplets at $\delta 6.18$ and $6.08 \mathrm{ppm}$, respectively. The coupling constants are ${ }^{3} J(\mathrm{~F}-\mathrm{H})=9.652 \mathrm{~Hz},{ }^{4} J(\mathrm{~F}-\mathrm{H})=7.302 \mathrm{~Hz}$, and ${ }^{3} J(\mathrm{~F}-\mathrm{H})$ $=9.564 \mathrm{~Hz},{ }^{4} J(\mathrm{~F}-\mathrm{H})=7.452 \mathrm{~Hz}$ respectively. These values are matching well with those of previously reported compounds. Therefore we can say that the selective exchange of hydrogen for fluorine in the para position is observed under formation of two new compounds $\mathrm{PhCOC}\left(4-\mathrm{C}_{6} \mathrm{~F}_{4} \mathrm{H}\right)$ and $\mathrm{C}_{6} \mathrm{~F}_{5} \mathrm{CO}\left(4-\mathrm{C}_{6} \mathrm{~F}_{4} \mathrm{H}\right)$.

The reaction of LSnH (20) with 4-fluoro benzophenone leads to the exclusively nucleophilic addition product $\mathrm{LSnOCHPh}\left(4-\mathrm{C}_{6} \mathrm{H}_{4} \mathrm{~F}\right)(39)$ (Scheme 4.10). There are no indications that the para fluorine is affected. The ${ }^{1} \mathrm{H}$ NMR spectra of 39 exhibits a singlet ( $\left.\delta 5.15 \mathrm{ppm}\right)$ which corresponds to the quaternary $\mathrm{CH}$ proton and the other signals are as expected. The ${ }^{19} \mathrm{~F}$ NMR resonance arises at $\delta-112.3 \mathrm{ppm}$ as a multiplet, also in the the ${ }^{119} \mathrm{Sn}$ NMR resonance of 39 arises a singlet at $\delta-212 \mathrm{ppm}$, which is similar to that of compound $\mathrm{LSnOCHPh}_{2}(\mathbf{2 3})(\delta-218$ ppm) (see Table 4.1). In the EI mass spectrum the molecular ion peak was observed at $\mathrm{m} / \mathrm{z}$

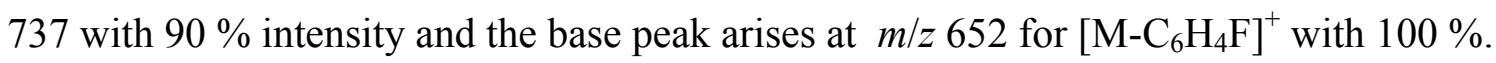


Treatment of $\mathrm{LSnH}$ with pentafluoro benzaldehyde leads quantitatively to the stannylene alkoxide $\mathrm{LSnOCH}_{2} \mathrm{C}_{6} \mathrm{~F}_{5}$ (40), with a $\mathrm{Sn}$ (II)-O- $\mathrm{CH}_{2}$ framework that is formed by nucleophilic hydride addition to the carbon of the carbonyl group (Scheme 4.10).

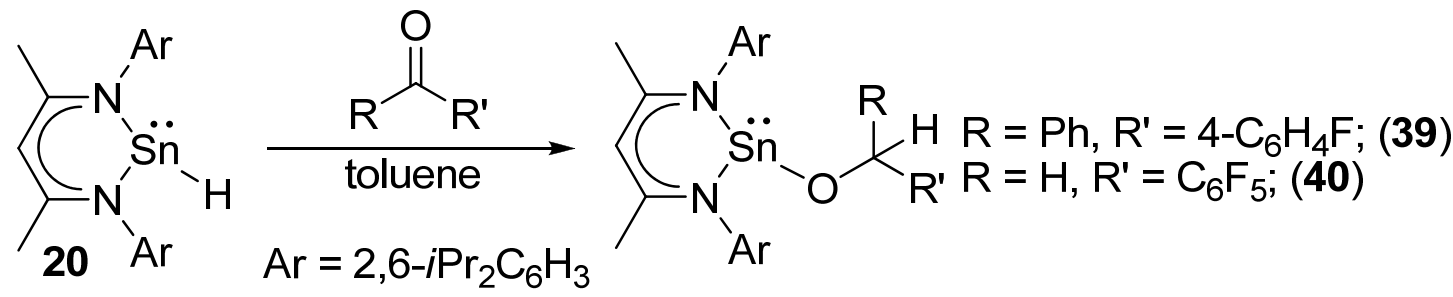

Scheme 4.10. Preparation of compounds 39 and 40

40 is a yellow solid, soluble in benzene, THF, $n$-hexane, and $n$-pentane and shows no decomposition on exposure to air. The ${ }^{1} \mathrm{H}$ NMR spectrum of $\mathbf{4 0}$ exhibits a singlet $(\delta 4.81$ ppm), which can be assigned to the $\mathrm{CH}_{2}$ protons and is flanked by $\mathrm{Sn}$ satellite lines $\left({ }^{3} J\left({ }^{119} \mathrm{Sn},{ }^{1} \mathrm{H}\right)=13.5 \mathrm{~Hz}\right)$. The ${ }^{119} \mathrm{Sn}$ NMR resonance of 40 arises at $\delta$-262 ppm. 40 crystallizes in the triclinic space group $P-1$ with one monomer in the asymmetric unit (Figure 4.9). The Sn-O bond lengths (2.0300(14) A) are comparable with those of compound $\mathrm{LSnOCH}_{2} \mathrm{Fc}$, (22) (2.0253(13) $\AA$ ). The $\mathrm{O}-\mathrm{C}$ bond distances of $\mathbf{4 0}$ and $\mathrm{LSnOCH}_{2} \mathrm{Fc}$ (22) are in a narrow range of each other (1.404(2) $\AA$ and 1.411(2) $\AA$ ).

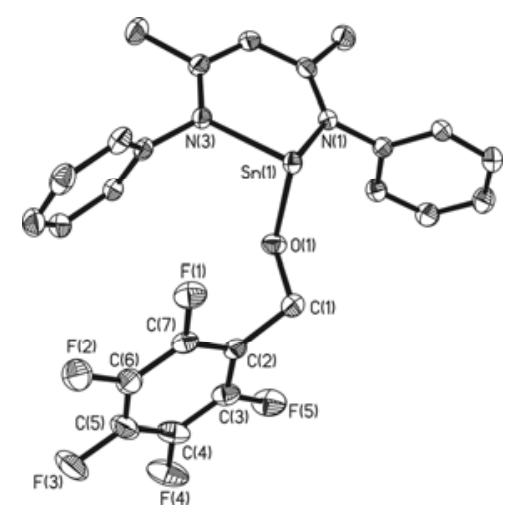

Figure 4.9. Molecular structure of 40. Thermal elipsoids are shown at 50\% probability. Isopropyl groups and $\mathrm{H}$ atoms are omitted for clarity reasons. Selected bond lengths $[\AA]$ and angles $\left[^{\circ}\right]$ : Sn1-O1 2.0300(14), O1-C1 1.404(2), Sn1-N1 2.1911(15), Sn1-N3 2.1920(15); Sn1-O1-C1 120.19(11), N1-Sn1-O1 91.85(6), N1-Sn1-N3 83.61(6). 
Table 4.1. ${ }^{119} \mathrm{Sn}$ NMR data of $\mathrm{Sn}(\mathrm{II})$ compounds

\begin{tabular}{|c|c|}
\hline Compound $^{*}$ & ${ }^{119} \mathrm{Sn} \operatorname{NMR}(\delta, \mathrm{ppm})$ \\
\hline $\mathrm{LSnCl}$ & -224 \\
\hline $\operatorname{LSnF}(\mathbf{1})$ & -371.50 \\
\hline LSnH (20) & -4.45 \\
\hline $\mathrm{LSnOC}(\mathrm{O}) \mathrm{H}(\mathbf{2 1})$ & -360 \\
\hline $\mathrm{LSnOCH}_{2} \mathrm{Fc}(\mathbf{2 2})$ & -262 \\
\hline $\mathrm{LSnOCHPh}_{2}(\mathbf{2 3})$ & -218 \\
\hline LSnOCHPh(2-Py) (24) & -286 \\
\hline $\mathrm{LSnOCH}(2-\mathrm{Py})_{2}(\mathbf{2 5})$ & -324 \\
\hline $\mathrm{LSnOCH}\left(\mathrm{C}_{3} \mathrm{H}_{5}\right)_{2}(\mathbf{2 6})$ & -190.50 \\
\hline $\mathrm{LSnC}\left(\mathrm{CH}_{2}\right) \mathrm{CO}_{2} \mathrm{Me}(\mathbf{2 7})$ & -93.28 \\
\hline $\mathrm{LSnC}\left(\mathrm{CH}_{2}\right) \mathrm{CO}_{2} \mathrm{Et}(\mathbf{2 8})$ & -89.02 \\
\hline $\mathrm{LSnC}(\mathrm{CHMe}) \mathrm{CO}_{2} \mathrm{Et}(\mathbf{2 9})$ & -90 \\
\hline $\mathrm{LSnC}\left(\mathrm{CHCO}_{2} \mathrm{Me}\right) \mathrm{CO}_{2} \mathrm{Me}(\mathbf{3 0})$ & $-132,-211$ \\
\hline $\mathrm{LSnC}\left(\mathrm{CHCO}_{2} \mathrm{Et}\right) \mathrm{CO}_{2} \mathrm{Et}(\mathbf{3 1})$ & $-130.36,-211.20$ \\
\hline $\mathrm{LSnC}\left(\mathrm{CHCO}_{2} \mathrm{Me}\right) \mathrm{CO}_{2} \mathrm{Et}(\mathbf{3 2})$ & $-123.27,-205.75$ \\
\hline $\mathrm{LSn}(i \operatorname{PrNCHN} i \operatorname{Pr})(\mathbf{3 3})$ & -177.69 \\
\hline LSn(CyNCHNCy) (34) & -175 \\
\hline $\mathrm{LSnOCH}\left(\mathrm{CF}_{3}\right) \mathrm{Ph}(\mathbf{3 5})$ & -252 \\
\hline $\mathrm{LSnOCH}\left(2-\mathrm{C}_{4} \mathrm{H}_{3} \mathrm{~S}\right) \mathrm{CF}_{3}(\mathbf{3 6})$ & -262 \\
\hline $\mathrm{LSnOCHPh}\left(\mathrm{C}_{6} \mathrm{~F}_{5}\right)(37)$ & -217 \\
\hline $\mathrm{LSnOCH}\left(\mathrm{C}_{6} \mathrm{~F}_{5}\right)_{2}(\mathbf{3 8})$ & -252 \\
\hline $\mathrm{LSnOCHPh}\left(4-\mathrm{C}_{6} \mathrm{H}_{4} \mathrm{~F}\right)(\mathbf{3 9})$ & -212 \\
\hline $\mathrm{LSnOCH}_{2} \mathrm{C}_{6} \mathrm{~F}_{5}(\mathbf{4 0})$ & -262 \\
\hline
\end{tabular}

${ }^{*} \mathrm{~L}=\mathrm{HC}(\mathrm{CMeNAr})_{2} ; \mathrm{Ar}=2,6-i \mathrm{Pr}_{2} \mathrm{C}_{6} \mathrm{H}_{3}$ 


\section{Chapter 5}

\section{Synthesis and Reactivity of Six-Membered N-Heterocyclic Germylene}

Since the pioneering work on the synthesis of isolable germylenes $\left(\mathrm{R}_{2} \mathrm{Ge}\right)$ reported by Lappert et al.$^{[105]}$ remarkable progress has been made in tuning the reactivity of the low coordinate Ge atom in germylenes through the presence of bulky and/or p-donor substituents

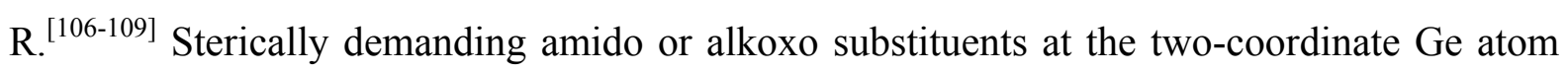
lead to derivatives which can serve as ligands with different donor-acceptor abilities in coordination compounds. ${ }^{[110-114]}$ Among the amido-substituted germylenes, the Nheterocyclic germylenes deserve particular attention, because they are versatile building blocks for the synthesis of germanium compounds with remarkable electronic features. Recent highlights include the synthesis of compounds with resonance-stabilized $\mathrm{X}-\mathrm{Ge}=\mathrm{Y}$ moieties $\left(\mathrm{X}=\mathrm{F}, \mathrm{Cl}, \mathrm{OH} ; \mathrm{Y}=\mathrm{S},{ }^{[172,206]} \mathrm{Se},{ }^{[207]} \mathrm{NR}^{[28]}\right)$ and the formation of the N-heterocyclic germanium(II) cation $\mathrm{LGe}^{+},{ }^{[208]}$ starting from the sterically encumbered $\beta$-diketiminatosubstituted chlorogermylene, $\mathrm{LGeCl}^{[146]}$

\subsection{Synthesis of Six-Membered N-Heterocyclic Germylene}

In chapter 3 we have already discussed the synthesis of $\mathrm{L}^{\prime} \mathrm{Ge}(\mathbf{1 9}), \quad\left(\mathrm{L}^{\prime}=\right.$ $\left.\mathrm{CH}\left\{\left(\mathrm{C}=\mathrm{CH}_{2}\right)(\mathrm{CMe})(\mathrm{NAr})_{2}\right\} ; \mathrm{Ar}=2,6-i \mathrm{Pr}_{2} \mathrm{C}_{6} \mathrm{H}_{3}\right)$ form $\mathrm{LGeH}$ (5) by a frustrated Lewis carbene borane pair under elimination of dihydrogen. The carbene and borane act as a proton and hydride scavenger respectively, and the formation of imidazolium cation with borate as a counter anion. The naked hydride cannot be a counter anion, therefore there is no reaction between the $\mathrm{LGeH}$ and NHC. We changed $\mathrm{LGeH}(5)$ to $\mathrm{LGeCl}$, where the chloride anion is a counter anion of the imidazolium cation. Herein we describe the synthesis of 19 from $\mathrm{LGeCl}$ and also from the LGeOTf as starting materials.

A toluene solution of one equivalent of $\mathrm{LGeCl}$ and one equivalent of 1,3-di-tertbutylimidazol-2-ylidene ${ }^{[191]}$ under stirring immediately changed the color from yellow to brown-red after a few minutes and a white precipitate is formed (Scheme 5.1). This is in contrast to the observation when a solution of one equivalent of $\mathrm{LGeCl}$ and one equivalent of 1,3-di-(2,4,6- $\left.\mathrm{Me}_{3} \mathrm{C}_{6} \mathrm{H}_{2}\right)$-2-ylidene was used. ${ }^{[209]}$ The reason are the different nucleophilic properties of NHCs. 


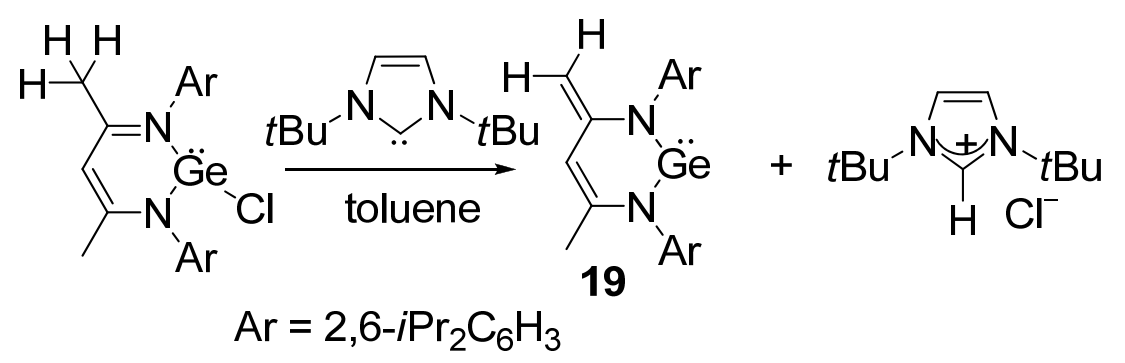

Scheme 5.1. Preparation of compound 19

The insoluble white precipitate was identified as the 1,3-di-tert-butylimidazolium chloride, and the soluble part as the germylene $\mathrm{L}^{\prime} \mathrm{Ge}(\mathbf{1 9})$. We postulate that the reaction proceeds via the cleavage of a $\mathrm{C}-\mathrm{H}$ methyl bond and the formation of the $\mathrm{C}-\mathrm{H}$ carbonium ion (Scheme 5.1). This type of $\mathrm{C}-\mathrm{H}$ cleavage reaction was reported for nucleophilic carbenes. ${ }^{[210]}$ The proof for the $\mathrm{C}-\mathrm{H}$ bond activation at the backbone methyl group of $\mathrm{LGeCl}$ by 1,3-di-tertbutylimidazol-2-ylidene is given herein by the isolation of 1,3-di-tert-butylimidazolium chloride. The previously reported germylene 19 was formed by the reaction of $\mathrm{LGeCl}$ with $\mathrm{LiN}\left(\mathrm{SiMe}_{3}\right)_{2} \cdot{ }^{[37]}$ Similar deprotonation reactions at the backbone have been observed for the borane stabilized germylene hydride, ${ }^{[28]}$ the tautomerization of an iminogermane bearing the $\beta$-diketiminato ligand, ${ }^{[29]}$ a cyclodiazaborane analogue,${ }^{[32]}$ and related calcium complexes. ${ }^{[33]}$ This is so far a unique example where the deprotonation reaction at the backbone has been demonstrated using 1,3-di-tert-butylimidazol-2-ylidene.

In the above discussed reaction the yield is not so high (65\%), it can be improved when instead of LGeCl, LGeOTf and NHC are used. The triflate anion $\left(\left[\mathrm{OSO}_{2} \mathrm{CF}_{3}\right]^{-}=\mathrm{OTf}\right)$ has served here as an excellent leaving group and the conversion is almost quantitative (Scheme $5.2)$.

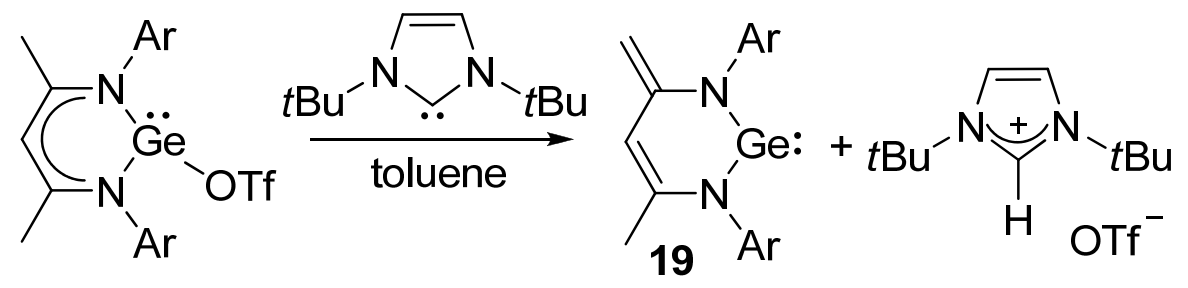

Scheme 5.2. Preparation of compound 19 


\subsection{Reaction of $L^{\prime} G e$ with Ammonia Borane}

The synthesis of L'Ge was achieved either by the dehydrogenation, dehydrohalogenation, or dehydrotriflation of the corresponding germanium(II) compounds $\mathrm{LGeH}, \mathrm{LGeCl}$, and LGeOTf respectively. Accordingly it may be possible that $\mathrm{L}^{\prime} \mathrm{Ge}$ (19) can react with dihydrogen to form LGeH (5). Furthermore it should be emphasized that di(amino)carbenes are inert towards dihydrogen, ${ }^{[211]}$ whereas the acyclic and cyclic alkyl(amino)carbenes undergo oxidative addition of dihydrogen at the carbene carbon atom. ${ }^{[212]}$ In addition dihydrogen reacts with digermynes ${ }^{[213]}$ and stannylene ${ }^{[214]}$ respectively. Consequently we selected germylene L'Ge (19) for the reaction with dihydrogen. Unfortunately this compound showed no reaction with dihydrogen under mild conditions. Then we used as an alternative source of dihydrogen ammonia borane the preferred precursor for the preparation of boron nitride. Ammonia borane $\left(\mathrm{AB}, \mathrm{H}_{3} \mathrm{NBH}_{3}\right)$, which has a $19.6 \%$ hydrogen content, has recently attracted great interest due to its potential application for chemical hydrogen storage. ${ }^{[215]}$ The $\mathrm{AB}$ molecule contains both hydridic $\mathrm{B}-\mathrm{H}$ and protic $\mathrm{N}-\mathrm{H}$ polarized bonds and a strong $\mathrm{B}-\mathrm{N}$ bond so that hydrogen release from solid $\mathrm{AB}$ is more favorable than dissociation into ammonia and diborane under non catalytic conditions.

The 1:1 reaction of L'Ge (19) with ammonia borane resulted in the formation of $\mathrm{LGeH}(\mathbf{5})$ (Scheme 5.3). Even an excess of ammonia borane leads only to $\mathbf{5}$. In the latter case we also observe the addition of one molecule of dihydrogen, without oxidation of germanium(II).

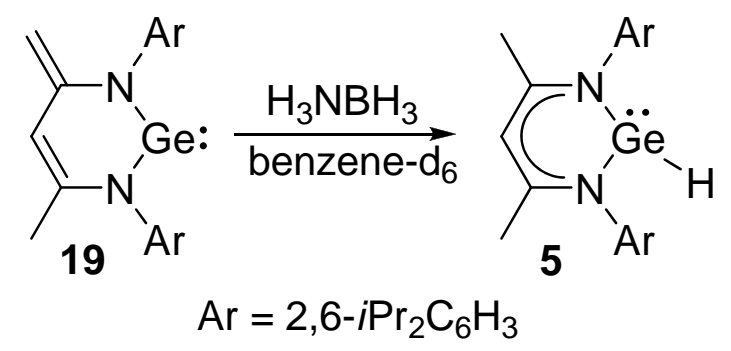

Scheme 5.3. Reaction of L'Ge with $\mathrm{H}_{3} \mathrm{NBH}_{3}$

Here it is mentioned that the reaction of $\mathrm{L}^{\prime} \mathrm{Ge}$ with $\mathrm{Me}_{3} \mathrm{~N} \cdot \mathrm{HCl}$ or1,3-di-(2,6-i $\left.\mathrm{Pr}_{2} \mathrm{C}_{6} \mathrm{H}_{3}\right)-2-$ ylidene hydrochloride salt leads to the $\mathrm{LGeCl} .^{[146]}$ 


\subsection{Reaction of NHC with Ammonia Borane}

In this context we did the reaction of ammonia borane with N-heterocyclic carbenes, 1,3-ditert-butylimidazol-2-ylidene. ${ }^{[191]}$ N-heterocyclic carbenes (NHCs) are well studied in terms of ligands (organometallic chemistry), organocatalysis including a number of reports of unique and unexpected reactivity. ${ }^{[86]}$ The hydrogenation energy for the conversion of 1,3-ditert-butylimidazol-2-ylidene is exothermic. In the absence of a catalyst 1,3-di-tertbutylimidazol-2-ylidene is inert towards hydrogen but in the presence of catalytic amounts of palladium or platinum a slow uptake of dihydrogen and formation of the 1,1-addition product is observed. ${ }^{[216]}$ The 1:1 reaction of ammonia borane with 1,3-di-tert-butylimidazol-2-ylidene at room temperature in the absence of any catalyst in toluene leads to compound $41{ }^{[216]}$ The CC-double bond present in 1,3-di-tert-butylimidazol-2-ylidene is unaffected even when we used an excess of ammonia borane. In the ${ }^{1} \mathrm{H}$ NMR there are only three resonances at 5.4, 4.2 , and $1.0 \mathrm{ppm}$ in the ratio of 1:1:9 and they are in accordance with an original sample. ${ }^{[216]}$ The conversion of 1,3-di-tert-butylimidazol-2-ylidene to 41 at room temperature is quantitative.

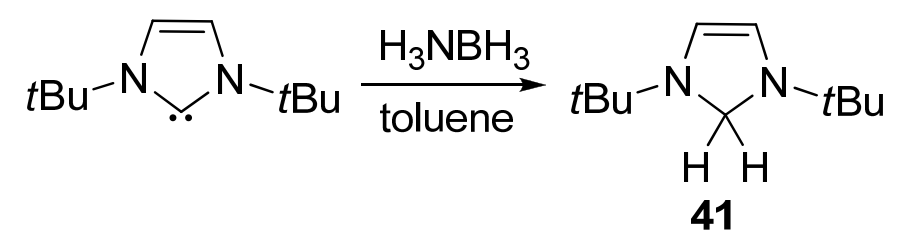

Scheme 5.4. Preparation of compound 41

\subsection{Reaction of $L^{\prime} G e$ with Ammonia}

In recent years we have been interested in the synthesis of low valent germanium compounds and reported on the preparation and structural characterization of $\mathrm{LGeCl}^{[146]}{ }^{2 \mathrm{LeF}},{ }^{[28]}$ $\mathrm{LGeOH},{ }^{[127]}$ and $\mathrm{LGeH}^{[79]}$ These small reactive molecules are ideal precursor for the preparation of heterobimetallic germylenes, which are active catalysts in olefin polymerization reactions. ${ }^{[217,218]}$ One important missing species in this series is the $\mathrm{LGeNH}_{2}$. To date, there is only one example known of a stable germanium(II) amide featuring the parent $\mathrm{NH}_{2}$ group. ${ }^{[137]}$ A conventional route for the preparation of $\mathrm{Ge}-\mathrm{NH}_{2}$ containing compounds is the ammonolysis of the corresponding halide. ${ }^{[139,143,144]}$ Therefore it was expected that the reaction of $\mathrm{LGeCl}$ with an excess of ammonia leads to the product $\mathrm{LGeNH}_{2}$. 
However, in a broad temperature range $\left(-40\right.$ to $\left.25^{\circ} \mathrm{C}\right)$ the starting material does not react with ammonia. From our previous experience for the preparation of $\mathrm{LGeOH}^{[127]}$ and $\mathrm{LAl}\left(\mathrm{NH}_{2}\right)_{2}{ }^{[145]}$ using $\mathrm{LGeCl}$, and $\mathrm{LAlCl}_{2}$ respectively we employed N-heterocyclic carbenes (NHC) as a $\mathrm{HCl}$ acceptor. Here we can follow the route for the preparation of $\mathrm{LGeNH}_{2}$ from $\mathrm{LGeCl}, \mathrm{NH}_{3}$, and 1,3-di-(2,6-i $\left.\operatorname{Pr}_{2} \mathrm{C}_{6} \mathrm{H}_{3}\right)$-2-ylidene. May be during the reaction there the intermediate $\mathrm{L}^{\prime} \mathrm{Ge}(\mathbf{1 9})$ is formed, and after that it reacts with ammonia. Here we react directly ammonia with $\mathrm{L}^{\prime} \mathrm{Ge}$, and the formation of $\mathrm{LGeNH}_{2}$ under cleavage of one of the $\mathrm{N}-\mathrm{H}$ bond occured.

The activation of the ammonia molecule has attracted considerable interest over the years. Although ammonia usually forms simple Lewis acid-base adducts with transition metal complexes, there are also some $\mathrm{N}-\mathrm{H}$ activations of ammonia via transition metal complexes known. ${ }^{[67,219]}$ In contrast to these studies the corresponding chemistry of stable main group molecular compounds for the activation of ammonia is virtually nonexistent. In 2007, the first example of ammonia splitting was reported by Bertrand et al. using stable carbenes. ${ }^{[212]}$ Very recently Power et al. reported the activation of ammonia by a carbene analogue $\mathrm{SnAr}_{2}$ $\left(\mathrm{Ar}=2,6\left(2,6-i \mathrm{Pr}_{2} \mathrm{C}_{6} \mathrm{H}_{3}\right)_{2} \mathrm{C}_{6} \mathrm{H}_{3}\right){ }^{[214]}$

Herein we show the N-H bond cleavage of ammonia on employing $\mathrm{L}^{\prime} \mathrm{Ge}\left(\mathrm{L}^{\prime}=\right.$ $\left.\mathrm{CH}\left\{\left(\mathrm{C}=\mathrm{CH}_{2}\right)(\mathrm{CMe})(\mathrm{NAr})_{2}\right\} ; \mathrm{Ar}=2,6-i \mathrm{Pr}_{2} \mathrm{C}_{6} \mathrm{H}_{3}\right) ;$ (19), which generates under mild conditions a terminal Ge- $\mathrm{NH}_{2}$ group (Scheme 5.5). The addition of an excess of dry ammonia gas to a red solution of $\mathbf{1 9}$ in toluene leads to a rapid change of the color to orange. The solution was evaporated and extracted with $n$-hexane. Concentration of the solution yielded yellow crystals of $\mathrm{LGeNH}_{2}$ (42). X-ray crystal structure analysis afforded a monomeric structure as illustrated in Figure 5.1. Surprisingly, $\mathbf{4 2}$ is monomeric in the solid state and what is even more striking, the $\mathrm{NH}_{2}$ group is not involved in any kind of hydrogen bonding as shown by X-ray structural analysis and IR spectroscopy.

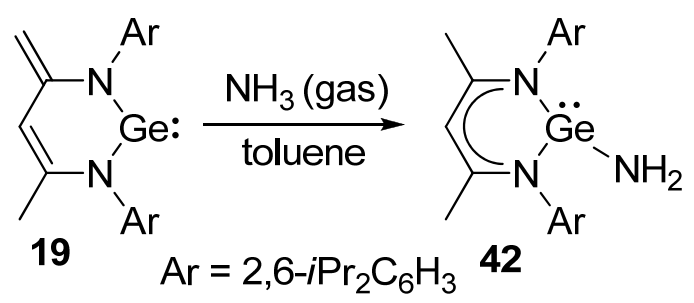

Scheme 5.5. Preparation of compound 42 
The IR spectrum displays two weak sharp bands at 3431 and $3333 \mathrm{~cm}^{-1}$ that are attributed to the two $\mathrm{N}-\mathrm{H}$ stretching modes of the $\mathrm{NH}_{2}$ group. These wave numbers are close to those at 3380 and $3308 \mathrm{~cm}^{-1}$ observed for $\left(\mathrm{Ar}^{*} \mathrm{GeNH}_{2}\right)_{2}\left(\mathrm{Ar}^{*}=2,6-\left(2,6-i \mathrm{Pr}_{2} \mathrm{C}_{6} \mathrm{H}_{3}\right)_{2} \mathrm{C}_{6} \mathrm{H}_{3}\right){ }^{[137]}$

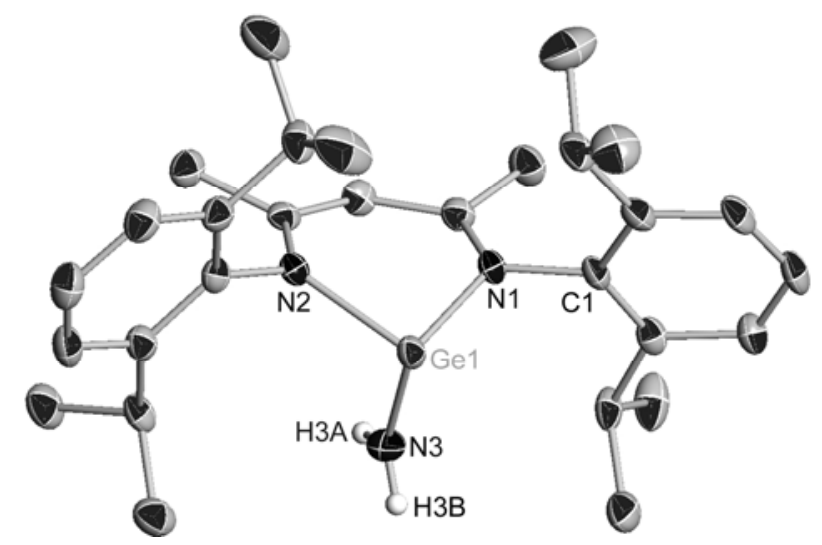

Figure 5.1. Anisotropic displacement parameters, depicted at the $50 \%$ probability level of $\mathbf{4 2}$. Hydrogen atoms, except those vital at the nitrogen atom N3, are omitted for clarity. Selected bond lengths $[\AA]$ and angles $\left[^{\circ}\right]$ : Ge1-N3 1.845(2), Ge1-N1 2.030(2), Ge1-N2 2.030(2); N1-Ge1-N2 88.60(8), N3-Ge1-N1 95.52(10), N3Ge1-N2 95.55(10).

\subsection{Synthesis of $\mathrm{LGeNHNH}_{2}$}

$\mathrm{L}^{\prime} \mathrm{Ge}$ cleaves one of the $\mathrm{N}-\mathrm{H}$ bonds of $\mathrm{NH}_{3}$ under formation of $\mathrm{LGeNH}_{2}$. Consequently we reacted $\mathrm{L}^{\prime} \mathrm{Ge}$ with hydrazine $\mathrm{NH}_{2} \mathrm{NH}_{2}$ and it leads to the germanium(II) substituted hydrazine derivative $\mathrm{LGeNHNH}_{2}$ (Scheme 5.6).

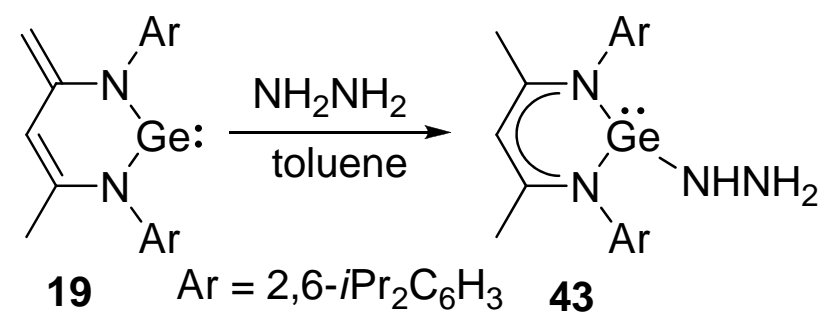

Scheme 5.6. Preparation of compound 43

Its composition was proven by ${ }^{1} \mathrm{H}$ NMR spectroscopy, EI mass spectroscopy, elemental analysis, and furthermore it was characterized by solid state X-ray structural analysis. The ${ }^{1} \mathrm{H}$ NMR spectrum shows a doublet and triplet resonance ( $\delta 3.16$ and $0.87 \mathrm{ppm})$, which corresponds to the two different amine protons of 43 . Compound 43 crystalizes from saturated $n$-hexane solution. 43 crystallizes in the triclinic space group $P-1$ with one monomer in the asymmetric unit (Figure 5.2). 


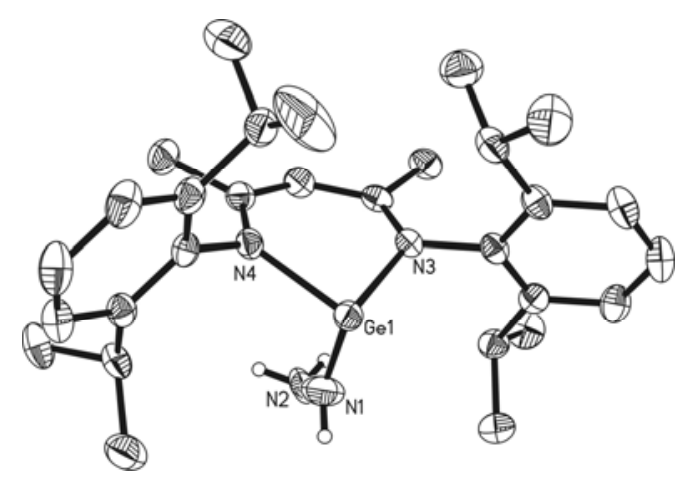

Figure 5.2. Molecular structure of 43. Anisotropic displacement parameters are depicted at the $50 \%$ probability level and all restrained refined hydrogen atoms are omitted for clarity. Selected bond lengths $[\AA]$ and angles $\left[{ }^{\circ}\right]$ are Ge1-N1 1.909(5), N1-N2 1.053(5), Ge1-N3 2.0141(19); N1-Ge1-N3 96.49(13), N2-N1-Ge1 134.6(3).

\subsection{Reactions of $L^{\prime}$ Ge with $\mathrm{O}-\mathrm{H}$ Bonded Compounds}

We have already discussed syntheses of $\mathrm{LGeNH}_{2}$ and $\mathrm{LGeNHNH}_{2}$ compounds by the cleavage of one N-H bond of ammonia and hydrazine respectively by $\mathrm{L}^{\prime} \mathrm{Ge}(19)\left(\mathrm{L}^{\prime}=\right.$ $\left.\mathrm{CH}\left\{\left(\mathrm{C}=\mathrm{CH}_{2}\right)(\mathrm{CMe})\left(2,6-i \mathrm{Pr}_{2} \mathrm{C}_{6} \mathrm{H}_{3} \mathrm{~N}\right)_{2}\right\}\right)$. Herein, we report on the synthesis of different heteroleptic germanium(II) compounds by the reaction of $\mathrm{L}^{\prime} \mathrm{Ge}(\mathbf{1 9})$ with $\mathrm{ROH}(\mathrm{R}=\mathrm{H}, \mathrm{Ph}$, $\mathrm{C}_{6} \mathrm{~F}_{5}, \mathrm{PhCO}$ ) under cleavage of the $\mathrm{O}-\mathrm{H}$ bond (Scheme 5.7).<smiles></smiles>

15<smiles>C=C1C=C(C)N([Ga])[Ge](=[Ge])N1[Al]</smiles>

19

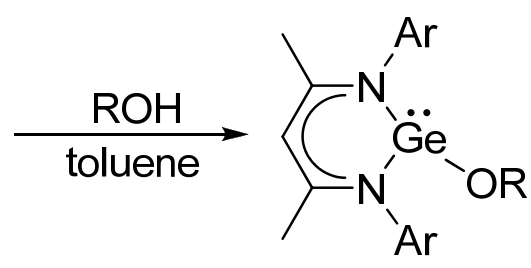

$$
\mathrm{R}=\mathrm{C}_{6} \mathrm{H}_{5} ;(\mathbf{4 4 )}
$$

$\mathrm{R}=\mathrm{C}_{6} \mathrm{~F}_{5} ;(45)$ $\mathrm{R}=\mathrm{C}_{6} \mathrm{H}_{5} \mathrm{CO} ;(46)$

Scheme 5.7. Preparation of compounds 15, 44, 45, and 46

The reaction of $\mathrm{L}^{\prime} \mathrm{Ge}$ with $\mathrm{H}_{2} \mathrm{O}$ in a 1:1 ratio at room temperature resulted in the formation of $\mathrm{LGeOH}$ (15) in almost quantitative yield (Scheme 5.7), and it is an alternative route for the preparation of $\mathrm{LGeOH}$. For a broader feasibility we extended the reaction of $\mathrm{L}^{\prime} \mathrm{Ge}$ with $\mathrm{PhOH}$ and $\mathrm{C}_{6} \mathrm{~F}_{5} \mathrm{OH}$ and obtained products 44 and 45 respectively (Scheme 5.7). 44 and 45 are colorless solids, which are soluble in $n$-hexane, $n$-pentane, THF, dichloromethane, benzene, and diethyl ether. 44 and $\mathbf{4 5}$ were characterized by ${ }^{1} \mathrm{H}$ NMR spectroscopy, EI mass spectrometry, elemental analysis, and X-ray single-crystal structural analysis. The ${ }^{1} \mathrm{H}$ NMR spectra of $\mathbf{4 4}$ and $\mathbf{4 5}$ show the expected pattern for the $\beta$-diketiminato ligand. The ${ }^{19} \mathrm{~F}$ NMR 
spectrum of 45 displays three resonances $(-158.8,-166.9,-175.2 \mathrm{ppm})$ for the ortho, meta, and para fluorine atoms respectively. The most intense peak in the EI mass spectra appeared at $m / z=491[\mathrm{M}-\mathrm{OPh}]^{+}$and $673[\mathrm{M}]^{+}$for compounds 44 and 45 respectively.

Compounds 44 and 45 crystallize in the orthorhombic Pnma and monoclinic $P 2_{1 / n}$ space group with one monomer in the asymmetric unit respectively. Single crystals of both 44 and 45 were obtained from saturated $n$-hexane solutions at $-32{ }^{\circ} \mathrm{C}$ after two days. These compounds are stable in the solid state as well as in solution for a long time without any decomposition under inert atmosphere. The coordination polyhedron around the germanium atom features a distorted trigonal pyramidal geometry (Figures 5.3 and 5.4). The germanium is attached to two nitrogen atoms from the backbone of the chelating ligand, and the oxygen atom from the $\mathrm{OPh}$ and $\mathrm{OC}_{6} \mathrm{~F}_{5}$ group respectively.

The Ge-N bond lengths and N-Ge-N angles are comparable with those of related compounds. The Ge-O bond length of 44 (1.860 $\AA)$ can be compared with that of LGeOH (15) (1.828 $\AA)^{[127]}$ but in 45 it is a slightly longer Ge-O bond (1.9515 $\AA$ ), when compared with the latter one.

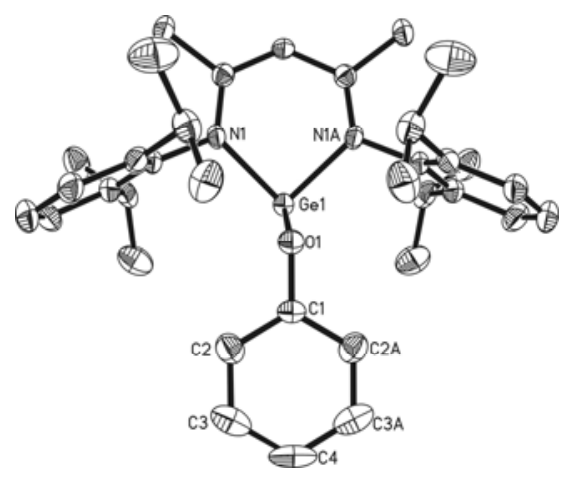

Figure 5.3. Molecular structure of 44. Selected bond lengths $[\AA]$ and angles $\left[^{\circ}\right]$; anisotropic displacement parameters are depicted at the $50 \%$ probability level and all restrained refined hydrogen atoms are omitted for clarity: Ge1-O1 1.860(4), Ge1-N1 2.008(3), N1-C17 1.314(5), O1-C1 1.360(7); N1-Ge1-N1A 88.67(16), N1Ge1-O1 93.88(12), Ge1-O1-C1 117.5(4). 


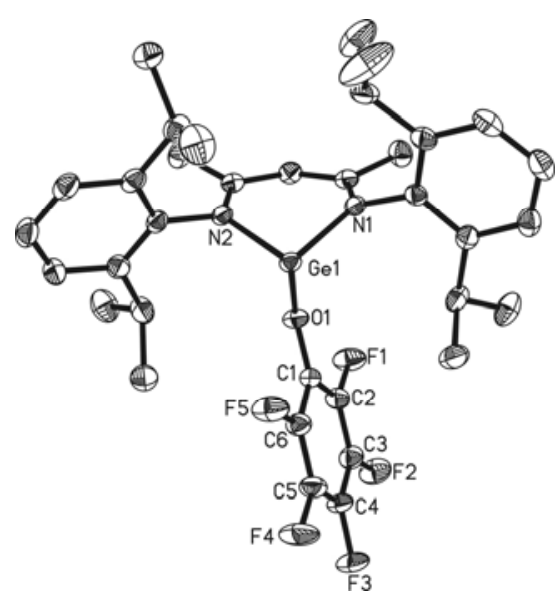

Figure 5.4. Molecular structure of 45. Selected bond lengths $[\AA]$ and angles $\left[^{\circ}\right]$; anisotropic displacement parameters are depicted at the $50 \%$ probability level and all restrained refined hydrogen atoms are omitted for clarity: Ge1-O1 1.9515(14), Ge1-N1 1.9785(16), N1-C19 1.338(2), O1-C1 1.319(2); N1-Ge1-N2 90.85(6), N1-Ge1-O1 91.11(6), Ge1-O1-C1 129.25(12).

Subsequently after the successful reaction of $\mathrm{L}^{\prime} \mathrm{Ge}$ with $\mathrm{H}_{2} \mathrm{O}, \mathrm{PhOH}$, and $\mathrm{C}_{6} \mathrm{~F}_{5} \mathrm{OH}$, respectively we used a carboxylic acid as precursor. Treatment of 19 with $\mathrm{PhCO}_{2} \mathrm{H}$ in toluene at room temperature after $1 \mathrm{~h}$ afforded the corresponding benzoic acid derivative, germylene benzoate $\mathrm{LGeOC}(\mathrm{O}) \mathrm{Ph}(46)$ in high yield (85\%) (Scheme 5.7). Compound 46 is thermally stable. No decomposition was observed at temperatures below the melting point $\left(167{ }^{\circ} \mathrm{C}\right)$ under an inert atmosphere. Compound $\mathbf{4 6}$ is a white solid that is soluble in benzene, toluene, $n$-hexane, and THF. 46 was characterized by ${ }^{1} \mathrm{H}$ NMR spectroscopy, EI mass spectrometry, elemental analysis, and X-ray structural analysis. The ${ }^{1} \mathrm{H}$ NMR spectrum of compound 46 shows a singlet $(\delta 5.16 \mathrm{ppm})$ for the $\gamma-\mathrm{CH}$ protons and two septets $(\delta 3.61,3.17 \mathrm{ppm})$ corresponding to the two different types of $\mathrm{CH}$ protons of the $i \mathrm{Pr}$ moieties. EI-MS of $\mathbf{4 6}$ gave the corresponding monomeric molecular ion peak $\mathrm{M}^{+}$at $\mathrm{m} / z 612$.

The molecular structure of $\mathbf{4 6}$ has been determined by single crystal X-ray diffraction analysis (Figure 5.5), which demonstrates that the complex exists as a monomer. Colorless crystals of 46 were obtained from a $n$-hexane solution at $-30{ }^{\circ} \mathrm{C}$ after one day in a freezer. Compound 47 crystallizes in the monoclinic space group $P 2_{1} / c$, with one monomer in the asymmetric unit. The coordination polyhedron around the germanium atom exhibits a distorted trigonal pyramidal geometry. The sum of angles around the germanium atom is $268.59^{\circ}$. The Ge-O bond distance (1.9462 $\AA$ ) in $\mathbf{4 6}$ is quite large when compared with that of $44(1.860 \AA)$ and it is almost similar to that of $45(1.9515 \AA)$. 


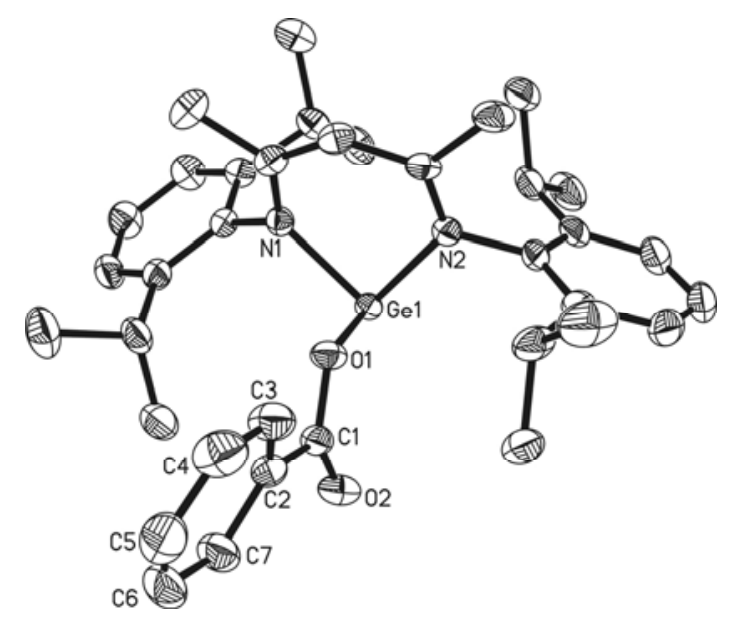

Figure 5.5. Molecular structure of 46. Selected bond lengths $[\AA]$ and angles $\left[^{\circ}\right]$; anisotropic displacement parameters are depicted at the $50 \%$ probability level and all restrained refined hydrogen atoms are omitted for clarity: Ge1-O1 1.9462(19), Ge1-N1 1.978(2), O1-C1 1.311(3), C1-O2 1.216(3); N1-Ge1-N2 90.48(9), N1Ge1-O1 89.43(8), O1-C1-O2 125.0(3).

\subsection{Reaction of $\mathrm{L}^{\prime} \mathrm{Ge}$ with $\mathrm{Ph}_{2} \mathrm{PCl}$}

Consequently we thought that the germylene 19 can be utilized for effecting substitution at the $\alpha$ carbon of the ligand back-bone. In order to introduce the $\mathrm{Ph}_{2} \mathrm{P}$ group at this position we have employed $\mathrm{Ph}_{2} \mathrm{PCl}$. A quantitative reaction has occurred when a solution of 19 in $n$ hexane is treated with $\mathrm{Ph}_{2} \mathrm{PCl}$ (Scheme 5.8). Product 47 was characterized by NMR and single crystal X-ray studies (Figure 5.6).

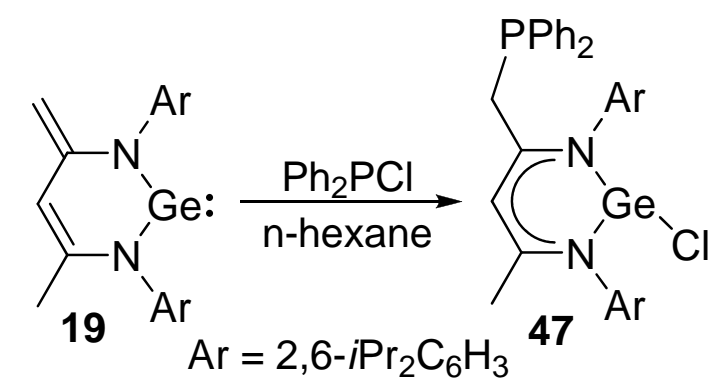

Scheme 5.8. Preparation of compound 47

As shown in Figure 5.6, the $N, N^{\prime}$-chelation mode of the ligand is retained and the $\mathrm{Ph}_{2} \mathrm{P}$ group does not interact with $\mathrm{Ge}(\mathrm{II})$. As with the previously reported $\beta$-diketiminate $\mathrm{Ge}(\mathrm{II})$ complexes, Ge deviates away from the plane of the ring and, Ge-N and $\mathrm{Ge}-\mathrm{Cl}$ bonds are also typical of such complexes. As given in Figure 5.6, all the $\mathrm{C}-\mathrm{C}$ and $\mathrm{CN}$ bond lengths of the $\beta$ diketiminate skeleton in $\mathbf{4 7}$ are almost equal. The ${ }^{31} \mathrm{P}$ NMR chemical shift of 47 (-13.47 ppm) is diagnostic of the coordination mode in this complex. Complex $\mathbf{4 7}$ is chiral and the chirality 
is due to the presence of out of the plane $\mathrm{Ge}-\mathrm{Cl}$ bond as well as the unsymmetrical nature of the ligand in $\mathbf{4 7}$. Interestingly, in $\mathbf{4 7}$ both enantiomers are present in the same unit cell (Figure $5.6)$.

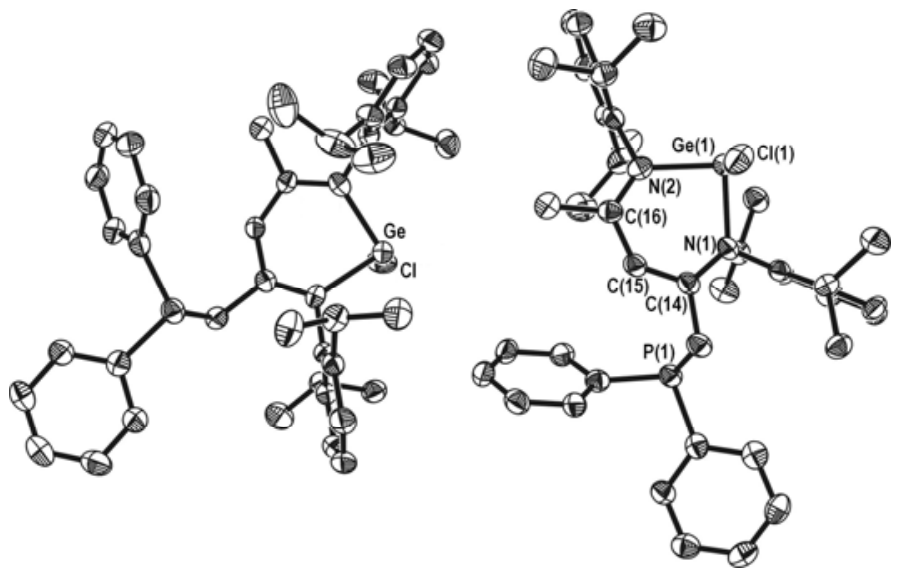

Figure 5.6. Molecular structure of 47 showing both the enantiomers. Relative orientations of enantiomers have been modified to obtain mirror images. Anisotropic displacement parameters are depicted at the $50 \%$ probability level and all restrained refined hydrogen atoms are omitted for clarity. Selected bond lengths $[\AA]$ and angles [ ${ }^{\circ}$ ]: Ge1-N1 1.984(3), Ge1-N2 1.967(3), Ge1-Cl1 2.3250(11), N1-C141.348(4), C14-C15 1.400(5), C15-C16 1.387(5), N(2)-C(16) 1.351(5); N2-Ge1-N1 91.35(12), N2-Ge1-Cl1 93.70(9), N1-Ge1-Cl1 94.61(8).

\subsection{Reaction of $L^{\prime} G e$ with $B\left(C_{6} F_{5}\right)_{3}$}

We have shown in the previous sections that the heterocyclic germylene 19 cleaves the $\mathrm{N}-\mathrm{H}$, and $\mathrm{O}-\mathrm{H}$ bonds of a variety of species at room temperature upon formation of stable heteroleptic germanium(II) compounds. Moreover Driess et al. had independently demonstrated that 19 has a dipolar character. ${ }^{[220]}$ Consequently we reacted 19 with $\mathrm{B}\left(\mathrm{C}_{6} \mathrm{~F}_{5}\right)_{3}$ at room temperature to afford the zwitterionic compound $\left[\mathrm{B}\left(\mathrm{C}_{6} \mathrm{~F}_{5}\right)_{3} \mathrm{~L}^{\prime \prime} \mathrm{Ge}\right](48)$, ( $\mathrm{L}^{\prime \prime}=$ $\left.\mathrm{CH}\left\{\left(\mathrm{CCH}_{2}\right)(\mathrm{CMe})(\mathrm{NAr})_{2}\right\} ; \mathrm{Ar}=2,6-i \mathrm{Pr}_{2} \mathrm{C}_{6} \mathrm{H}_{3}\right)$ containing a germylene moiety (Scheme 5.9).

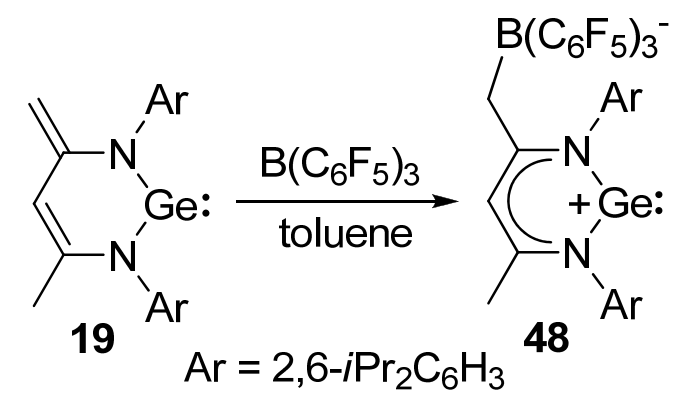

Scheme 5.9. Preparation of compound 48 
Only two reports on the synthesis of germanium(II) cations are in the current literature, one was reported by Power et al. where they also used a $\beta$-diketiminate ligand. ${ }^{[208,221]}$ Compound 48 was isolated as air and moisture sensitive pale yellow crystals in $78 \%$ yield, which are insoluble in common hydrocarbon solvents but highly soluble in THF. In this solvent there is no equilibrium between 19 and $\mathbf{4 8}$ observed in contrast to the isoelectronic silicon analogue of 19. ${ }^{[222]} 48$ crystallizes in the triclinic space group $P-1$ and consists of a planar sixmembered $\mathrm{C}_{3} \mathrm{~N}_{2} \mathrm{Ge}$ ring with an exocyclic $\mathrm{B}\left(\mathrm{C}_{6} \mathrm{~F}_{5}\right)_{3}$ group attached to $\mathrm{C} 13$ (Figure 5.7). The $\beta$-diketiminate $\mathrm{N}-\mathrm{C}$ and $\mathrm{C}-\mathrm{C}$ ring bond lengths have average values $(1.36$ and $1.39 \AA)$ and are shorter than those of $\mathbf{1 9}$, indicative for the delocalization of the $\pi$-electrons. The Ge-N bond lengths $\left(1.873(2)\right.$ and 1.881(2) $\AA$ ) are comparable with those of the cation [LGe] ${ }^{+}{ }^{[208]}$ The ${ }^{1} \mathrm{H}$ NMR spectrum exhibits a signal for the $\gamma$-CH proton at $6.50 \mathrm{ppm}$, which differs from that of germylene 19 (5.43 ppm). The ${ }^{11} \mathrm{~B}$ NMR spectrum shows a singlet resonance at $14.82 \mathrm{ppm}$ and the ${ }^{19} \mathrm{~F}$ NMR spectrum displays three resonances $(-131.6,-167.2$, and$163.40 \mathrm{ppm}$ ) for the ortho, para, and meta fluorine atoms respectively.

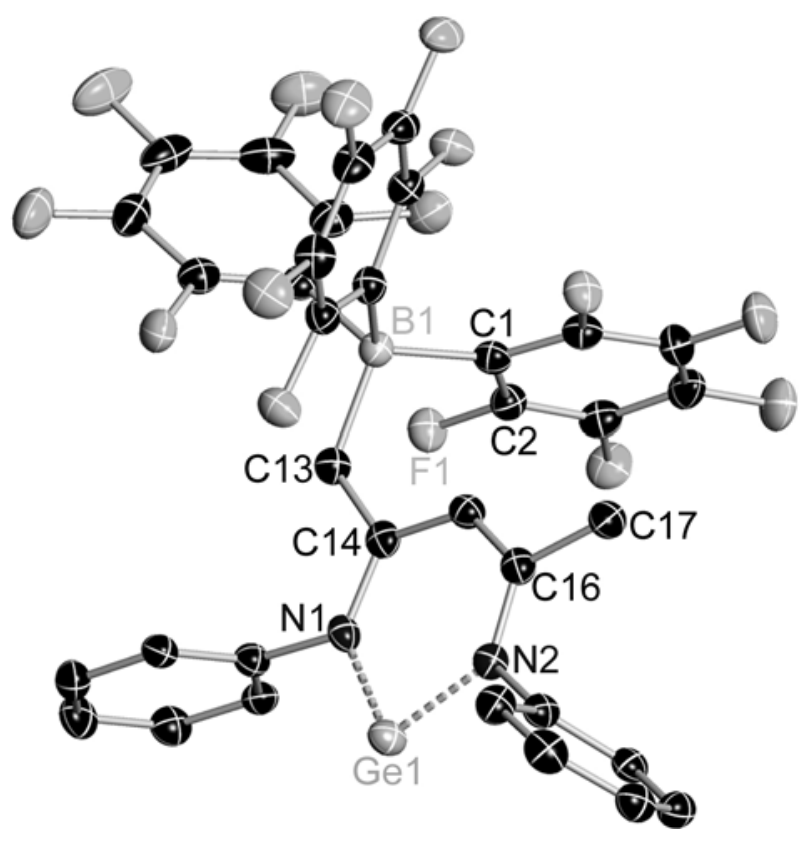

Figure 5.7. Molecular structure of 48. Anisotropic displacement parameters are depicted at the $50 \%$ probability level and all restrained refined hydrogen atoms and isopropyl groups are omitted for clarity. Selected bond lengths $[\AA]$ and angles $\left[^{\circ}\right]$ : Ge1-N1 1.845(2), Ge1-N2 2.030(2), C14-C13 1.845(2), C13-B1 1.845(2), C16C17 2.030(2), B1-C1 2.030(2); N1-Ge1-N2 88.60(8), C14-C13-B1 95.52(10), C13-B1-C1 95.55(10). 
Compound 19 might be compared with the monomeric aluminum(I), LA1, ${ }^{[13]}$ although the difference of the two species is one proton. This is demonstrated in the reactivity of $\mathbf{3}$ and LAl, respectively, with $\mathrm{B}\left(\mathrm{C}_{6} \mathrm{~F}_{5}\right)_{3}$ (Scheme 5.10). 19 assembled as a zwitterionic germanium(II) species 48 and the LAl forms a compound with a boron-aluminum bond. ${ }^{[223]}$

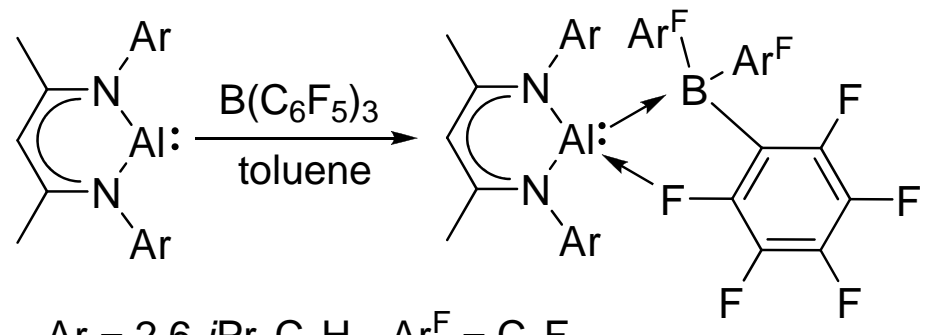

$$
\mathrm{Ar}=2,6-i \mathrm{Pr}_{2} \mathrm{C}_{6} \mathrm{H}_{3}, \mathrm{Ar}^{\mathrm{F}}=\mathrm{C}_{6} \mathrm{~F}_{5}
$$

Scheme 5.10. Reaction of $\mathrm{LAl}$ with $\mathrm{B}\left(\mathrm{C}_{6} \mathrm{~F}_{5}\right)_{3}$

Preliminary studies of $\mathbf{4 8}$ with 1,3-di-tert-butylimidazol-2-ylidene (NHC) show the deprotonation of the second methyl group from the backbone with formation of compound $\mathrm{B}\left(\mathrm{C}_{6} \mathrm{~F}_{5}\right)_{3} \mathrm{~L}^{\prime \prime \prime} \mathrm{Ge}(49),\left(\mathrm{L}^{\prime \prime \prime}=\mathrm{CH}\left\{\left(\mathrm{CCH}_{2}\right)\left(\mathrm{C}=\mathrm{CH}_{2}\right)(\mathrm{NAr})_{2}\right\} ; \mathrm{Ar}=2,6-i \mathrm{Pr}_{2} \mathrm{C}_{6} \mathrm{H}_{3}\right)$ under complete conversion (Scheme 5.11). The 1,3-di-tert-butylimidazol-2-ylidene reacts (Schemes 3.10, 5.1, 5.2, and 5.11) under formation of the imidazolium cation. However, the counter anion is different. The neutral compound 19 is formed to give the anion $\left[\mathrm{HB}\left(\mathrm{C}_{6} \mathrm{~F}_{5}\right)_{3}\right]^{-}, \mathrm{Cl}^{-}$, or OTf repectively, whereas in $\mathbf{4 9}$ we assume that the borate anion forms a stable ion pair with the imidazolium cation.

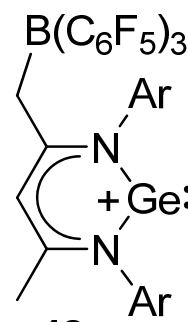

48

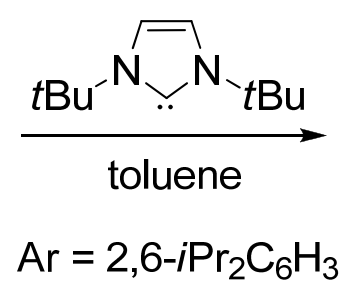

Scheme 5.11. Preparation of compound 49

The anion of 49 gives rise to the expected resonance with the $\gamma-\mathrm{CH}$ proton at $5.87 \mathrm{ppm}$, which is comparable with that of 19. The deprotonation of the second methyl group leads to the exocyclic carbon-carbon double bond. The two vinylic protons exhibit two nonequivalent singlets $(\delta 3.63,2.96 \mathrm{ppm})$, indicating that the germanium(II) atom is neutral in 
49. This is the first time we have shown that the double $\mathrm{C}-\mathrm{H}$ bond activation of the two methyl groups at the ligand backbone has occurred by NHC. So far there is only one $\mathrm{C}-\mathrm{H}$ bond activation of the methyl group at the ligand backbone reported. ${ }^{[28-33]}$ Unfortunately we were not able to prove this result by an X-ray crystal structural analysis of $\mathbf{4 9}$, due to the lack of single crystals. 


\section{Chapter 6}

\section{Reactivity of Six-Membered N-Heterocyclic Silylene}

In 2006 Driess et al. reported the synthesis of compound L'Si (50) (L' = $\left.\mathrm{CH}\left\{\left(\mathrm{C}=\mathrm{CH}_{2}\right)(\mathrm{CMe})(\mathrm{NAr})_{2}\right\} ; \mathrm{Ar}=2,6-i \mathrm{Pr}_{2} \mathrm{C}_{6} \mathrm{H}_{3}\right) .{ }^{[36]}$ In the past 20 years, the chemistry of stable N-heterocyclic silylenes (NHSs) has attracted much attention. ${ }^{[10,92,93,99,224,225]}$ Recently, we reported the synthesis and structures of the first monomeric heteroleptic silylene $\left[\left\{\mathrm{PhC}(\mathrm{N} t \mathrm{Bu})_{2}\right\} \mathrm{SiCl}\right],{ }^{[41]}$ and the derivatives $\left[\left\{\mathrm{PhC}(\mathrm{N} t \mathrm{Bu})_{2}\right\} \mathrm{SiNMe}_{2}\right],\left[\left\{\mathrm{PhC}(\mathrm{N} t \mathrm{Bu})_{2}\right\} \mathrm{SiR}\right](\mathrm{R}$ $=\mathrm{O} t \mathrm{Bu}, \quad \mathrm{O} i \mathrm{Pr}, \quad$ and $\left.\quad \mathrm{P} i \mathrm{Pr}_{2}\right),{ }^{[103]} \quad\left[\left\{\mathrm{PhC}(\mathrm{N} t \mathrm{Bu})_{2}\right\} \mathrm{Si}(\mathrm{S}) \mathrm{S} t \mathrm{Bu}\right],{ }^{[226]}$ and $\left[\left\{\mathrm{PhC}(\mathrm{N} t \mathrm{Bu})_{2}\right\} \mathrm{SiO} t \mathrm{Bu}\right] \mathrm{Fe}(\mathrm{CO})_{4} \cdot{ }^{[227]}$ In this year our group reported the synthesis of $\mathrm{SiCl}_{2}$ coordinated by N-heterocyclic carbene and reactivity towards diphenyl acetylene. ${ }^{[42]}$ The preparation of $\mathrm{NHC} \cdot \mathrm{SiCl}_{2}$ might have a similar impact in molecular silicon(II) chemistry as $\mathrm{GeCl}_{2}$.dioxane in the chemistry of divalent germanium. ${ }^{[228]}$ Moreover we have also prepared molecular $\mathrm{Si}(\mathrm{I})-\mathrm{Si}(\mathrm{I})$ compound, $\left[\left\{\mathrm{PhC}(\mathrm{N} t \mathrm{Bu})_{2}\right\} \mathrm{Si}\right]_{2}$ from the reduction of heteroleptic trichlorosilane $\left[\left\{\mathrm{PhC}(\mathrm{N} t \mathrm{Bu})_{2}\right\} \mathrm{SiCl}_{3}\right] .{ }^{[229]}$ Driess et al. showed the variety of reactivity ${ }^{[163,222,}$ ${ }^{230]}$ of L'Si (50). Some of the reactivity of $\mathbf{5 0}$ mimic the previously reported $\beta$-diketiminate substituted aluminum(I) LA1. ${ }^{[30,231]}$ Herein we describe the reaction of silylene L'Si (50) $\left(\mathrm{L}^{\prime}=\right.$ $\left.\mathrm{CH}\left\{\left(\mathrm{C}=\mathrm{CH}_{2}\right)(\mathrm{CMe})(\mathrm{NAr})_{2}\right\} ; \mathrm{Ar}=2,6-i \mathrm{Pr}_{2} \mathrm{C}_{6} \mathrm{H}_{3}\right)$ with ammonia, hydrazine, N-methyl hydrazine, and diphenyl hydrazone respectively, and found a striking difference with the reactions using L'Ge (19).

\subsection{Activation of Ammonia}

The activation of $\mathrm{N}-\mathrm{H}$ bonds of ammonia is rare. The first example of ammonia splitting by transition metals under the formation of an amido hydride complex was only recently reported by Hartwig and coworkers. ${ }^{[67]}$ Studies on the corresponding chemistry with main group compounds are explored to a lesser extent. In 2007 Bertrand et al. showed that the facile splitting of ammonia by nucleophilic activation at a single carbon center is possible. ${ }^{[212]}$ Last year Power et al. ${ }^{[214]}$ and Stephan et al. ${ }^{[184(\mathrm{c})]}$ independently showed, that the activation of ammonia occurred, when a diarylstannylene or a frustrated Lewis pair are involved. In general oxidative addition of the $\mathrm{N}-\mathrm{H}$ bond of ammonia to unsaturated species could be very important to generate new products. For example, the catalytic addition of ammonia to olefins and the coupling of ammonia with arenes are considered to be two of the ten greatest current challenges for catalytic chemistry. 
Herein we report the oxidative addition of ammonia at the silicon(II) center of L'Si (50) which occurs under mild conditions with the formation of $\mathrm{L}^{\prime} \mathrm{Si}(\mathrm{H}) \mathrm{NH}_{2}$. The addition of an excess of dry ammonia gas to a yellow solution of $\mathbf{5 0}$ in toluene leads to a rapid change of the yellow color to colorless (Scheme 6.1). The solution was evaporated and extracted with $n$ hexane. Concentration of the solution yielded colorless crystals of $\mathrm{L}^{\prime} \mathrm{Si}(\mathrm{H}) \mathrm{NH}_{2}(\mathbf{5 1})$ in $90 \%$ yield. Compound $\mathbf{5 1}$ is stable in the solid state as well as in solution for a long time without any decomposition under inert atmosphere.

Its composition was proven by multinuclear NMR and IR spectroscopy, mass spectrometry, and elemental $(\mathrm{C}, \mathrm{H}, \mathrm{N})$ analysis. The ${ }^{1} \mathrm{H}$ and ${ }^{29} \mathrm{Si} \mathrm{NMR}$ spectra revealed that $\mathbf{5 1}$ has a $\mathrm{Si}(\mathrm{H}) \mathrm{NH}_{2}$ moiety. The $\mathrm{Si}-\mathrm{H}$ proton shows a triplet $(\delta 4.97 \mathrm{ppm})$ with a coupling constant of ${ }^{3} J_{\mathrm{H}-\mathrm{H}}=4.5 \mathrm{~Hz}$. Thus the ${ }^{29} \mathrm{Si}\left\{{ }^{1} \mathrm{H}\right\}$ proton decoupled spectrum exhibits a singlet resonance $(\delta-$ $45.00 \mathrm{ppm})$ while the proton coupled spectrum shows a doublet resonance $(\delta-43.74$ and $46.27 \mathrm{ppm}$ ) with a coupling constant of $J_{\mathrm{Si}-\mathrm{H}}=251.72 \mathrm{~Hz}$. In addition the ${ }^{1} \mathrm{H}$ NMR spectrum exhibits the expected pattern for the ligand with a singlet of the $\gamma-\mathrm{CH}$ proton at $5.31 \mathrm{ppm}$. The IR spectrum displays two weak sharp bands $\left(\tilde{v}=3477\right.$ and $\left.3382 \mathrm{~cm}^{-1}\right)$ that are attributed to the $\mathrm{NH}_{2}$ group and a band at $2633 \mathrm{~cm}^{-1}$ for the $\mathrm{Si}-\mathrm{H}$ bond. The most intense peak in the EI mass spectrum appeared at $m / z=446\left[\mathrm{M}-\mathrm{CH}_{3}\right]^{+}$, and the signal at $m / z=461(15 \%)$ was assigned to the molecular ion $[\mathrm{M}]^{+}$.<smiles>C=C1C=C(C)N([Al])[SiH2]N1[AlH2]</smiles><smiles>C=C1C=C(C)N([Al])[Si](N)(N)N1[Al]</smiles>

50 $\mathrm{Ar}=2,6-\mathrm{Pr}_{2} \mathrm{C}_{6} \mathrm{H}_{3} \quad \mathbf{5 1}$

Scheme 6.1. Preparation of compound 51

Suitable crystals for X-ray structural analysis were obtained from a saturated $n$-hexane solution at $-32{ }^{\circ} \mathrm{C}$ after two days. Compound 51 crystallizes in the orthorhombic space group $\mathrm{Pbca}$ with one monomer in the asymmetric unit. X-ray crystal structure analysis afforded monomer in the solid state shown in Figure 6.1. Surprisingly, in $\mathbf{5 1}$ the $\mathrm{NH}_{2}$ group is not involved in any kind of hydrogen bonding as shown by X-ray structural analysis and IR spectroscopy. The coordination polyhedron around the silicon atom features a distorted 
tetrahedral geometry. The silicon is attached to two nitrogen atoms from the backbone of the chelating ligand, the $\mathrm{NH}_{2}$ group, and a hydrogen atom.

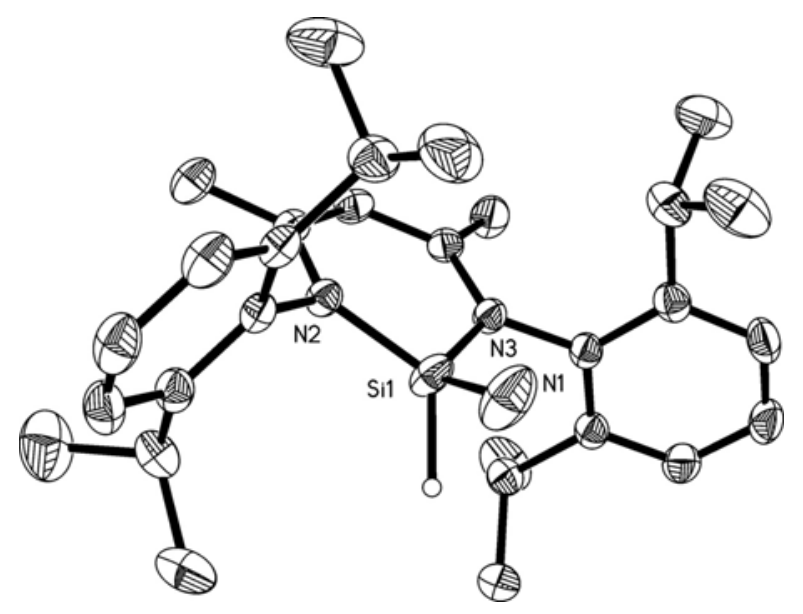

Figure 6.1. Thermal ellipsoid (50\%) drawing of 51. Hydrogen atoms, except those at silicon, are not shown for clarity. Selected bond lengths $[\AA]$ and angles $\left[^{\circ}\right]$ : Sil-N1 1.679(2), Si1-N2 1.679(2), Si1-N3 1.730(2); N1Si1-N2 120.20(19), N1-Si1-N3 114.58(11), N2-Si1-N3 114.58(11).

A noteworthy feature of compound $\mathbf{5 1}$ is the $\mathrm{Si}(\mathrm{H}) \mathrm{NH}_{2}$ moiety ( $\mathrm{Si}-\mathrm{N} 1.653(3) \AA$ ) ; the $\mathrm{Si}-\mathrm{N} 1$ bond length can be compared with the slightly longer $\mathrm{Si}-\mathrm{N}$ bond lengths in $\mathrm{RSi}\left(\mathrm{NH}_{2}\right)_{3}{ }^{[140,142]}$ $\left(\mathrm{R}=2,4,6-\mathrm{Ph}_{3} \mathrm{C}_{6} \mathrm{H}_{2}\right.$ av 1.709(7) $\AA, \mathrm{R}=2,6-i \mathrm{Pr}_{2} \mathrm{C}_{6} \mathrm{H}_{3} \mathrm{NSiMe}_{3}$ av 1.709(2) $\AA$, and $\mathrm{R}=2,4,6-$ $t \mathrm{BuC}_{6} \mathrm{H}_{2} \mathrm{O}$ av 1.692(6) $\AA$ ). Recently it was reported by Driess et al. that L'Si adds water under formation of the intermediate $\mathrm{L}^{\prime} \mathrm{Si}(\mathrm{H}) \mathrm{OH}$ or $\mathrm{LSiOH}[\mathrm{L}=\mathrm{HC}\{(\mathrm{CMe})(2,6-$ $\left.\left.i \operatorname{Pr}_{2} \mathrm{C}_{6} \mathrm{H}_{3} \mathrm{~N}\right)\right\}_{2}$ ]. However this intermediate cannot be isolated and reacts further with L'Si in contrast to compound $\mathbf{5 1} .^{[230(\mathrm{a})]}$

\subsection{Insertion of Silylene into N-H bond of Hydrazines}

Hydrazine and its derivatives are important molecules in the field of organic and inorganic chemistry since the discovery of a new indole synthesis in 1883 by Emil Fischer. ${ }^{[232]}$ Hydrazine is also very well known as an intermediate of nitrogenase in the field of biochemistry. ${ }^{[233]}$ The conversion of $\mathrm{N}_{2}$ to ammonia by enzymatic or chemical methods (via the intermediates of diimine and hydrazine) results in the cleavage of the N-N bond. ${ }^{[234,235]}$ In literature the cleavage of the N-N bond of hydrazine is well documented by a variety of model systems, while the $\mathrm{N}-\mathrm{H}$ bond cleavage of hydrazine is rare. ${ }^{[236]}$ On the metal surface the N-H bond cleavage of hydrazine is reported ${ }^{[237]}$ and substitution reactions of the hydrogen atoms of hydrazine are well known. ${ }^{[238]}$ To the best of our knowledge no insertion reaction at 
the N-H bond of hydrazine has been reported. Herein we describe the reaction of silylene L'Si (50) with hydrazine, N-methyl hydrazine, and diphenyl hydrazone respectively.

The addition of hydrazine to a yellow solution of $\mathbf{5 0}$ in toluene leads to a rapid vanishing of the yellow color (Scheme 6.2). The colorless solution was evaporated and extracted with $n$ hexane. Concentration of the solution yielded colorless crystals of $\mathrm{LSi}(\mathrm{H}) \mathrm{NHNH}_{2}(\mathbf{5 2})$ in 85 $\%$ yield. The composition of $\mathbf{5 2}$ was proven by multinuclear NMR spectroscopy, mass spectrometry, and elemental $(\mathrm{C}, \mathrm{H}, \mathrm{N})$ analysis. The ${ }^{1} \mathrm{H}$ and ${ }^{29} \mathrm{Si}$ NMR spectra revealed that 52 has a $\mathrm{Si}(\mathrm{H}) \mathrm{NHNH}_{2}$ moiety. The $\mathrm{Si}-\mathrm{H}$ proton shows a doublet $(\delta 5.05 \mathrm{ppm})$ with a coupling constant of ${ }^{3} J(\mathrm{H}-\mathrm{H})=4.34 \mathrm{~Hz}$. The ${ }^{29} \mathrm{Si}$ NMR spectrum exhibits a doublet resonance $(\delta-42.33$ and $-44.95 \mathrm{ppm})$ with a coupling constant of ${ }^{2} J(\mathrm{Si}-\mathrm{H})=260.5 \mathrm{~Hz}$. Each doublet further splits into a doublet with the coupling constant of ${ }^{3} J(\mathrm{Si}-\mathrm{H})=12.73 \mathrm{~Hz}$ due to the $\mathrm{NH}$ proton. In addition the ${ }^{1} \mathrm{H}$ NMR spectrum exhibits the expected pattern for the ligand (L) with a singlet and doublet of the $\gamma-\mathrm{CH}$ and $\mathrm{NH}_{2}$ protons at 5.30 and $1.86 \mathrm{ppm}$, respectively. The most intense peak in the EI mass spectrum appeared at $\mathrm{m} / z=461[\mathrm{M}-$ $\left.\mathrm{CH}_{3}\right]^{+}$, and the signal at $m / z=476(10 \%)$ was assigned to the molecular ion $[\mathrm{M}]^{+}$.

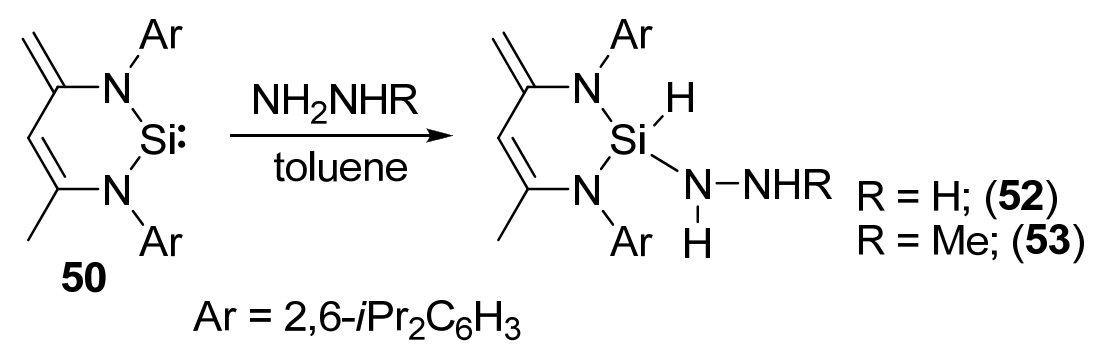

Scheme 6.2. Preparation of compounds 52 and 53

Furthermore we were interested in the selective $\mathrm{N}-\mathrm{H}$ bond cleavage of the non-symmetric hydrazine derivative. Therefore we selected $\mathrm{N}$-methyl hydrazine, $\left(\mathrm{MeNHNH}_{2}\right)$. Compound 50 reacts with $\mathrm{N}$-methyl hydrazine in toluene at room temperature to form exclusively LSi(H)NHNHMe (53). This was confirmed by ${ }^{1} \mathrm{H}$ NMR in solution. The ${ }^{1} \mathrm{H}$ NMR spectrum shows a doublet and quartet resonance ( $\delta 2.25$ and $1.73 \mathrm{ppm})$, which corresponds to the two different amine protons of 53. In addition there are no signals corresponding to the $\mathrm{NH}_{2}$ protons, therefore we excluded the formation of another isomer of 53. Also the ${ }^{29} \mathrm{Si} \mathrm{NMR}$ spectrum shows a doublet resonance $(\delta-42.31$ and $-44.97 \mathrm{ppm})$ with a coupling constant of 
${ }^{2} J(\mathrm{Si}-\mathrm{H})=263.8 \mathrm{~Hz}$. Each doublet again is splitted into a doublet with the coupling constant of ${ }^{3} J(\mathrm{Si}-\mathrm{H})=13.17 \mathrm{~Hz}$. This coupling constant is similar to that in compound $\mathbf{5 3}$. Furthermore each doublet again splits into a doublet due to its neighboring proton of the NHMe group, with the coupling constant of ${ }^{4} J(\mathrm{Si}-\mathrm{H})=2.34 \mathrm{~Hz}$. The most intense peak in the EI mass spectrum appeared at $m / z=475\left[\mathrm{M}-\mathrm{CH}_{3}\right]^{+}$, and the signal at $m / z=490(25 \%)$ was assigned to the molecular ion $[\mathrm{M}]^{+}$.

Suitable crystals for X-ray structural analysis were obtained from a saturated $n$-hexane solution at $-32{ }^{\circ} \mathrm{C}$ after two days. Compound $\mathbf{5 2}$ crystallizes in the monoclinic space group $P 2_{1} / n$ with one monomer in the asymmetric unit. Surprisingly, in $\mathbf{5 2}$ the $\mathrm{NHNH}_{2}$ group is not involved in any kind of hydrogen bonding as shown by X-ray structural analysis (Figure 6.2) and IR spectroscopy. The silicon is attached to two nitrogen atoms from the backbone of the chelating ligand, $\mathrm{NHNH}_{2}$ group, and a hydrogen atom.

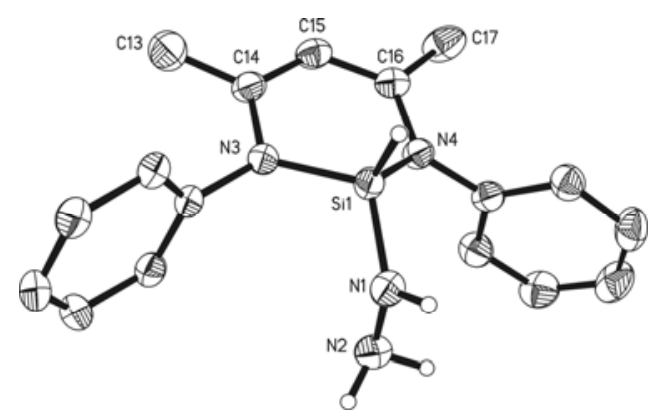

Figure 6.2. Thermal ellipsoid (50\%) drawing of 52. Isopropyl groups of the phenyl rings and hydrogen atoms, except those at silicon and at nitrogen that were found and refined freely, are not shown for clarity. Selected bond lengths $[\AA]$ and angles $\left[^{\circ}\right]$ : Si1-N1 1.679(2), N1-N2 1.435(3), Si1-N3 1.730(2); Si1-N1-N2 120.20(19), N1-Si1-N3 114.58(11).

\section{3. [1+4] Cycloaddition of Silylene with Diphenyl Hydrazone}

The reaction of $\mathbf{5 0}$ with an equimolar amount of diphenyl hydrazone in toluene proceeds rapidly even in a broad temperature range $\left(-78{ }^{\circ} \mathrm{C}\right.$ to $\left.\mathrm{rt}\right)$ under the formation of the remarkably dearomatized siloxi-indolin-1-amine $\mathbf{5 4}$ in almost quantitative yield (Scheme 6.3). To our surprise there was no insertion of the silicon(II) center into the $\mathrm{N}-\mathrm{H}$ bond of the $\mathrm{NH}_{2}$ group during the reaction. Even after a longer period of time $\mathbf{5 4}$ is not converted into an aromatized silyl amine tautomer or any other product. 


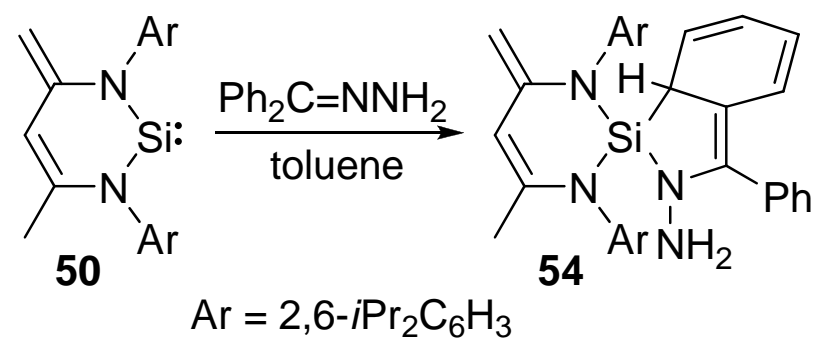

Scheme 6.3. Preparation of compound 54

Very recently Driess et al. reported that $\mathbf{5 0}$ reacts with benzophenone under formation of a dearomatized product at low temperatures. ${ }^{[230(\mathrm{e})]}$ However this product converted to the aromatic silyl ether tautomer. This result is in contrast to the formation of compound $\mathbf{5 4} .54$ is a yellow solid soluble in benzene, THF, $n$-hexane, and $n$-pentane, respectively, and shows no decomposition on exposure to dry air. $\mathbf{5 4}$ was characterized by multinuclear spectroscopy, EI mass spectrometry, elemental analysis, and X-ray structural analysis. The ${ }^{1} \mathrm{H}$ NMR spectrum of 54 shows a singlet ( $\delta 2.97 \mathrm{ppm})$ which can be assigned to the $\mathrm{NH}_{2}$ protons. The ${ }^{29} \mathrm{Si}$ NMR spectrum exhibits a doublet resonance $(\delta-19.87$ and $-20.07 \mathrm{ppm})$ with a coupling constant of ${ }^{3} J(\mathrm{Si}-\mathrm{H})=20.4 \mathrm{~Hz}$.

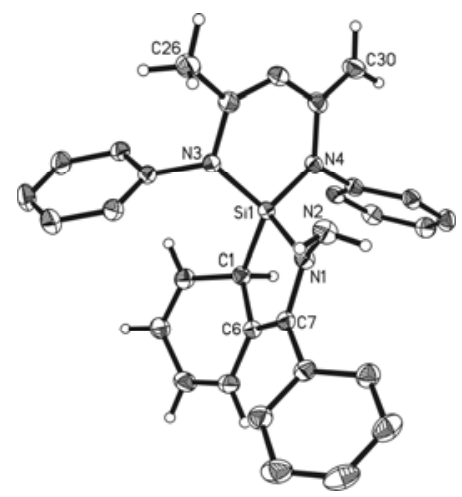

Figure 6.3. Thermal ellipsoid (50\%) drawing of 54. Isopropyl groups of the phenyl rings and hydrogen atoms, except those at N2, C26 and at C30 and non aromatics, that were found and refined freely, are not shown for clarity. Selected bond lengths $[\AA]$ and angles $\left[^{\circ}\right]$ : Si1-N1 1.7480(12), N1-N2 1.4322(15), Si1-C1 1.8786(13), C1-C6 1.5260(16), C6-C7 1.3596(17); Si1-N1-N2 117.46(8), Si1-C1-C6 103.70(8), N3-Si1-N4 103.44(9).

Suitable crystals for X-ray structural analysis were obtained from a hot saturated $n$-hexane solution of 54 after keeping this solution overnight at room temperature. 54 crystallizes in the triclinic space group $P-1$, with one molecule of $\mathbf{5 4}$ in the asymmetric unit. 54 exists as a monomer in the solid state (Figure 6.3). There are no intermolecular hydrogen bonds 
observed in the crystal lattice. The coordination polyhedron around the silicon atom comprises two nitrogen atoms from the supporting ligand, one carbon atom from the dearomatized phenyl group, and one nitrogen atom of the hydrazone moiety, featuring a distorted tetrahedral geometry. Compound $\mathbf{5 4}$ represents a spirocyclic compound with the silicon atom at the spiro center within a six-membered $\mathrm{SiN}_{2} \mathrm{C}_{3}$ ring and a five-membered $\mathrm{SiNC}_{3}$ ring, which are orthogonal arranged to each other.

Compounds 52 and $\mathbf{5 4}$ exhibit the Si-N-NH$H_{2}$ moiety (Si1-N1 1.679(2) $\AA$ for $\mathbf{5 2}$ and Si1-N1 1.7480(12) A for 54); the Si1-N1 bond lengths can be compared with that in 51 (1.653(3) $\AA$ ) and those in $\mathrm{RSi}\left(\mathrm{NH}_{2}\right)_{3}{ }^{[140,142]}\left(\mathrm{R}=2,4,6-\mathrm{Ph}_{3} \mathrm{C}_{6} \mathrm{H}_{2}\right.$ av 1.709(7) $\AA, \mathrm{R}=2,6-i \mathrm{Pr}_{2} \mathrm{C}_{6} \mathrm{H}_{3} \mathrm{NSiMe}_{3}$ av 1.709(2) $\AA$, and $\mathrm{R}=2,4,6-t \mathrm{Bu}_{3} \mathrm{C}_{6} \mathrm{H}_{2} \mathrm{O}$ av 1.692(6) $\AA$ ). The $\mathrm{N}-\mathrm{N}$ bond lengths in $\mathbf{5 2}$ and 54 (N1-N2; 1.435 (3) $\AA$ and 1.4322(15) $\AA$ ) are comparable with that in $\mathrm{LGeN}\left(\mathrm{CO}_{2} \mathrm{Et}\right) \mathrm{NHCO}_{2} \mathrm{Et}$, (14) $(1.418(14) \AA)$. 


\section{Chapter 7}

\section{Diazo Alkane an Effective Reagent for Unprecedented Reactions with Compounds of Low Valent Group 14 Elements}

The reaction of diazoalkane with transition metals has a long history regarding the utility of carbene transfer for cyclopropanation of olefins. ${ }^{[239]}$ Moreover metal carbene complexes play an important role in olefin metathesis, ${ }^{[240]} \mathrm{C}-\mathrm{H}$ bond activation, ${ }^{[241]}$ and the synthesis of reactive ylides. ${ }^{[242]}$ In all these examples diazoalkane either interacts with the metal center to form the metal diazoalkane complex or subsequently releases dinitrogen from diazoalkane to form the metal carbene complex. Compared to this, the reaction of diazoalkane with main group metals is scarcely known in literature. ${ }^{[243]}$

\subsection{Reactions of Diazo Compounds with LGeH}

We have already described the synthesis of $\mathrm{LGeH}(5) ;\left(\mathrm{L}=\mathrm{HC}(\mathrm{CMeNAr})_{2} ; \mathrm{Ar}=2,6-\right.$ $\left.i \mathrm{Pr}_{2} \mathrm{C}_{6} \mathrm{H}_{3}\right)$ and its reactivity towards multiple bonded carbon-carbon, carbon-oxygen, and nitrogen-nitrogen systems, and also with elemental sulfur, nitrous oxide, and frustrated Lewis carbene-borane pair. Subsequently we became interested in studying the reactivity of $\mathbf{5}$ with diazoalkane. Some reactions between metal hydrides and diazoalkanes have been reported. ${ }^{[244]}$ We expected from the reaction of diazoalkane with $\mathbf{5}$ one of the following products: $\mathrm{LGe}(\mathrm{H}) \mathrm{NNCHR}, \mathrm{LGeNNCH} \mathrm{R}_{2}, \mathrm{LGe}(\mathrm{H}) \mathrm{CHR}$, or $\mathrm{LGeCH}_{2} \mathrm{R}$. To our surprise none of these species was formed.

Treatment of $\mathbf{5}$ with ethyl diazoacetate and trimethylsilyl diazomethane, respectively leads to the first stable germanium(II) substituted hydrazone derivatives, LGeN(H)NCHR, (R = $\mathrm{CO}_{2} \mathrm{Et}(\mathbf{5 5}), \quad \mathrm{SiMe}_{3}(\mathbf{5 6})$ ) in high yield (Scheme 7.1). The reaction proceeds through the unprecedented end-on insertion of diazoalkane into the Ge-H bond. The initial dinitrogen elimination followed by insertion or oxidative addition reaction is generally accepted. Accordingly, the end-on $\mathrm{N}_{2} \mathrm{CHR}$ insertion into the $\mathrm{Ge}-\mathrm{H}$ bond unambiguously reveals the initial interaction between the Ge center and the terminal nitrogen atom of the diazo group followed by hydrogen transfer from the germanium center to nitrogen. This type of reaction is, to the best of our knowledge, so far unknown. 
Compounds 55 and $\mathbf{5 6}$ have been well-characterized by spectroscopic, analytic, and X-ray crystallographic measurements. The ${ }^{1} \mathrm{H}$ NMR spectra of $\mathbf{5 5}$ and $\mathbf{5 6}$ exhibit a singlet $(\delta 7.25$ and $6.37 \mathrm{ppm}$ ) which can be assigned to the $\mathrm{N}-H$ proton. In addition 55 and $\mathbf{5 6}$ show singlet resonances ( $\delta 6.62,4.91 \mathrm{ppm}$ and $\delta 6.44$ and $4.96 \mathrm{ppm})$ which corresponds to the imine and $\gamma$-CH protons. The IR spectrum of 55 exhibits bands at 3188, 2700 and $1641 \mathrm{~cm}^{-1}$, which are assigned to the $\mathrm{N}-\mathrm{H}, \mathrm{C}-\mathrm{H}$ and $\mathrm{C}=\mathrm{O}$ stretching frequencies.

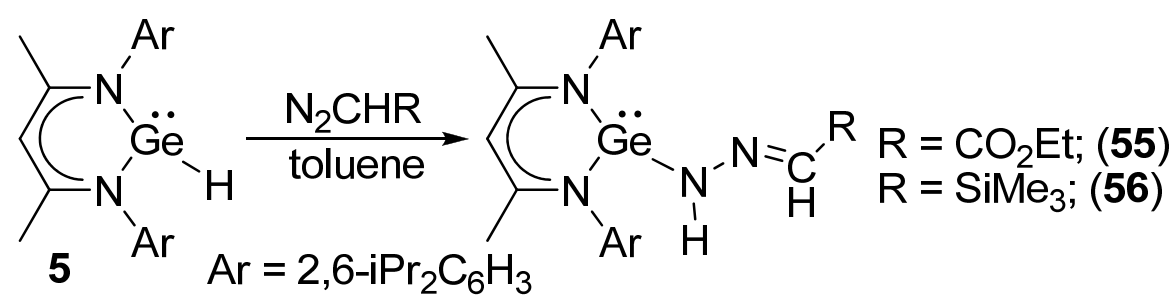

Scheme 7.1. Preparation of compounds 55 and 56

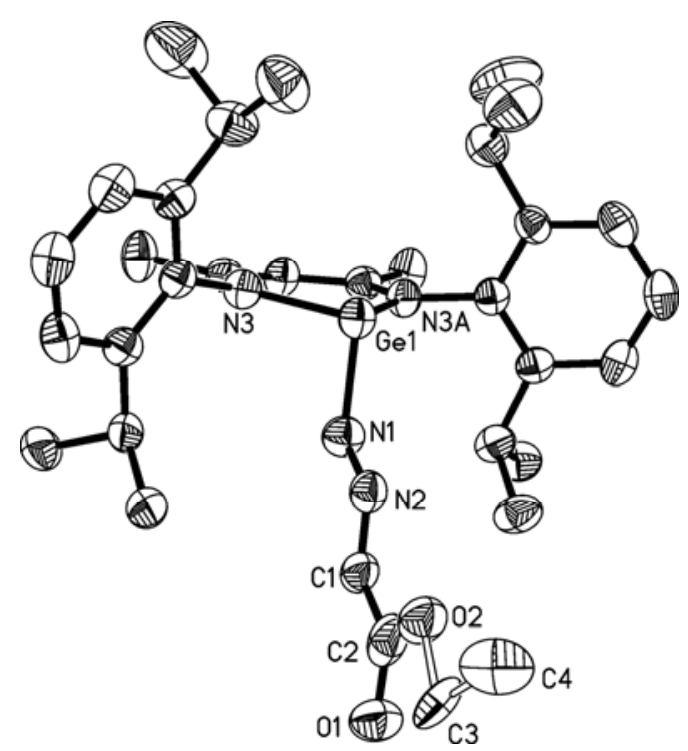

Figure 7.1. Molecular structure of 55. Thermal elipsoids are shown at 50\% probability. $\mathrm{H}$ atoms are omitted for clarity reasons. Selected bond lengths $[\AA]$ and angles $\left[{ }^{\circ}\right]$ : Ge1-N1 1.915(7), N1-N2 1.344(10), N2-C1 1.273(10), Ge1-N3 2.015(5), Ge1-N3A 2.015(5); Ge1-N1-N2 117.0(6), N1-N2-C1 118.7(7), N3-Ge1-N3A $94.2(2)$.

Both 55 and 56 are soluble in benzene, THF, $n$-hexane, and $n$-pentane, respectively and show no decomposition on exposure to air. In the solid state 55 and $\mathbf{5 6}$ are yellow colored. 55 crystallizes from saturated toluene solution at room temperature after one day in the tetragonal space group $P 4_{2} / n c m$, with one monomer in the asymmetric unit (Figure 7.1). 
Compound 56 has one $\mathrm{SiMe}_{3}$ group and displays a singlet in the ${ }^{29} \mathrm{Si}$ NMR spectrum $(\delta 6.28$ ppm). 56 crystallizes from saturated $n$-hexane solution at room temperature after two days in the monoclinic space group $C 2 / m$, with one monomer in the asymmetric unit (Figure 7.2).

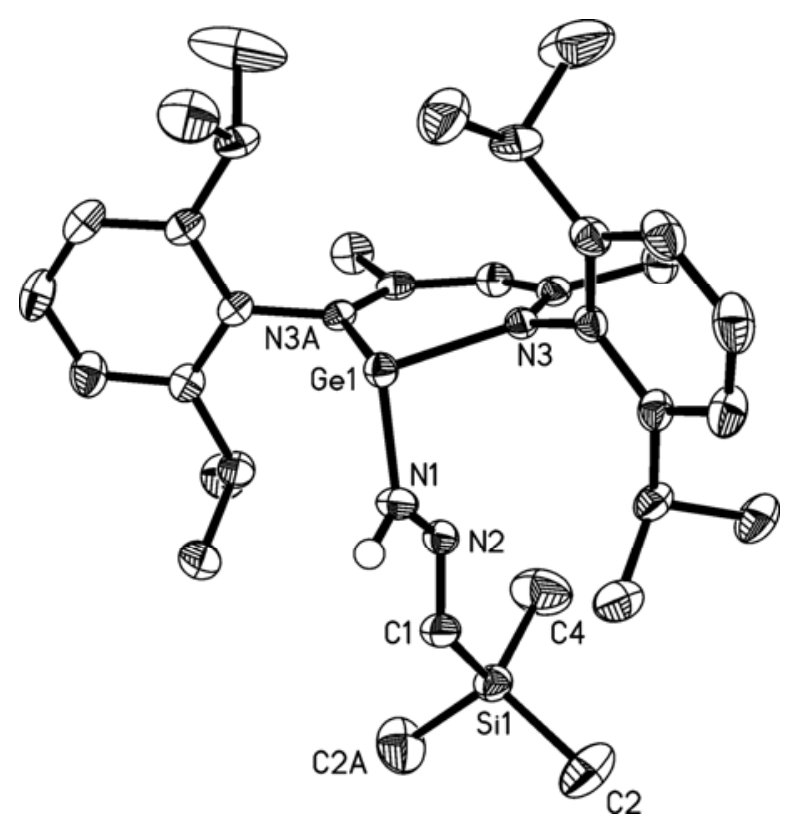

Figure 7.2. Molecular structure of 56. Thermal elipsoids are shown at 50\% probability. $\mathrm{H}$ atoms except N1-H are omitted for clarity reasons. Selected bond lengths $[\AA]$ and angles $\left[{ }^{\circ}\right]$ : Ge1-N1 1.878(3), N1-N2 1.351(4), N2-C1 1.303(4), Ge1-N3 1.9913(17), Ge1-N3A 1.9913(17); Ge1-N1-N2 130.6(2), N1-N2-C1 117.4(3), N3Ge1-N3A 90.41(10).

\subsection{Reaction of Trimethylsilyl Diazomethane with LGeMe}

In 2002 our group reported the reaction of LGeMe with trimethylsilyl azide under the formation of $\mathrm{L}^{\prime} \mathrm{Ge}(\mathrm{Me})\left(\mathrm{NHSiMe}_{3}\right)$ with a subsequent elimination of dinitrogen gas. ${ }^{[29]}$ Trimethylsilyl azide and trimethylsilyl diazomethane are isoelectronic, therefore we performed the reaction of LGeMe with the latter one, which resulted in the formation of [L'Ge(Me)(NHNCHSiMe 3$)]$ (57), instead of nitrogen elimination and creation of $\mathrm{LGe}(\mathrm{Me}) \mathrm{CHSiMe}_{3}$ (Scheme 7.2). The reaction proceeds with migration of a hydrogen atom from a methyl group of the ligand backbone to the end-on nitrogen atom of the diazo group. In the ${ }^{1} \mathrm{H}$ NMR spectrum of $\mathbf{5 9}$ the resonances clearly show the existence of $\mathrm{N} H(\delta 6.76 \mathrm{ppm})$ $\mathrm{CH}(\delta 5.78 \mathrm{ppm})$, and the $\mathrm{CH}_{2}$ moiety ( $\delta 3.83$ and $\left.3.21 \mathrm{ppm}\right)$. 


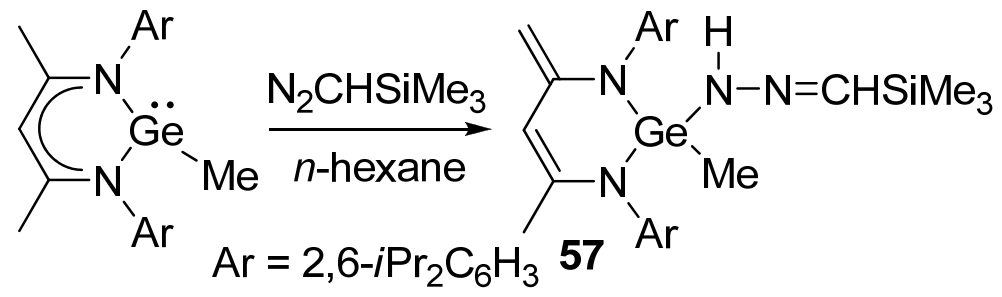

Scheme 7.2. Preparation of compound 57

This result also unambiguously reveals the initial interaction between the Ge(II) center and the terminal nitrogen atom of the diazo group followed by the hydrogen transfer from the methyl group of ligand backbone to the end-on nitrogen of the diazo group. The formation of 57 may be compared with that of [ $\left.\mathrm{L}^{\prime} \mathrm{Ge}\left(\mathrm{NHSiMe}_{3}\right)_{2}\right]$ (18) from the reaction of $\mathrm{LGeH}(\mathbf{5})$ and trimethylsilyl azide (Chapter 3, Scheme 3.8). In both compounds an oxidation of the Ge(II) to Ge(IV) occurred and a migration of a hydrogen atom from a methyl group of the ligand backbone to the nitrogen atom is observed.

\subsection{Reaction of Trimethylsilyl Diazomethane with L'Ge}

We have already discussed the cleavage of $\mathrm{N}-\mathrm{H}$ and $\mathrm{O}-\mathrm{H}$ bonds using the heterocyclic germylene L'Ge (19). Now we reacted 19 with trimethylsilyl diazomethane under formation of the diazogermylene $\left.\mathrm{LGeC}_{(} \mathrm{N}_{2}\right) \mathrm{SiMe}_{3}(\mathbf{5 8})$ by a C-H bond cleavage (Scheme 7.3). Here it is also noted that the trimethylsilyl diazomethane reacts with $\mathrm{LGeH}(5)$ with an unprecedented end-on nitrogen insertion of diazo nitrogen into the $\mathrm{Ge}-\mathrm{H}$ bond under formation of germanium(II)-substituted hydrazones 55 and 56 (Scheme 7.1). 58 is readily soluble in hydrocarbon and ether solvents and has been fully characterized.

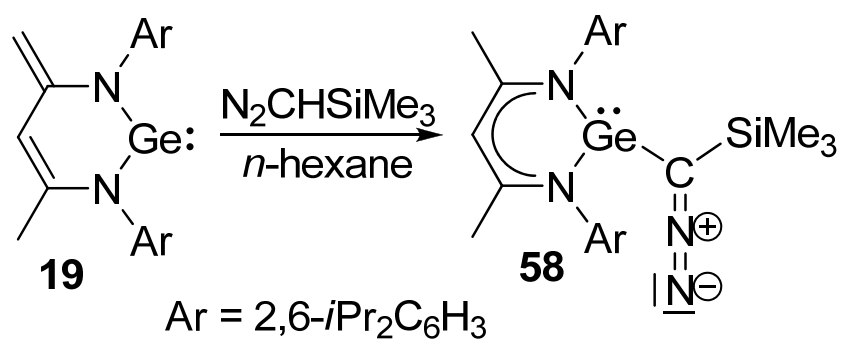

Scheme 7.3. Preparation of compound 58

The germanium complex 58 crystallizes in the triclinic space group $P-1$ with one molecule in the asymmetric unit (Figure 7.3). The germanium atom is coordinate to two nitrogen atoms of the ligand $(\mathrm{N} 1, \mathrm{~N} 2)$ and to the carbon atom (C25) of the diazolyl group. The Ge1-C25 
(1.9969(14) $\AA$ ) and Si1-C25 (1.8345(15) $\AA$ ) bond lengths are close to the normal values for $\sigma$ bonds of 1.99 and $1.87 \AA$ respectively. Also the C25-N3 bond length with 1.3037(19) $\AA$ is in the range of a carbon-nitrogen double bond $(1.29 \AA)$ merely the N3-N4 bond length $(1.1458(19) \AA)$ lies between a nitrogen-nitrogen double and triple bond $(\mathrm{N}=\mathrm{N} 1.25 \AA, \mathrm{N} \equiv \mathrm{N}$ $1.10 \AA$ ). The Ge1-C25-Sil angle of $118.37(7)^{\circ}$ is consistent with a $s p^{2}$ hybridized carbon atom (C25). These values are closely related to those of a reported diazogermylene compound. ${ }^{[245]}$ Compound $\mathbf{5 8}$ has one $\mathrm{SiMe}_{3}$ group and displays a singlet in the ${ }^{29} \mathrm{Si}$ NMR spectrum $(\delta-2.43 \mathrm{ppm})$.

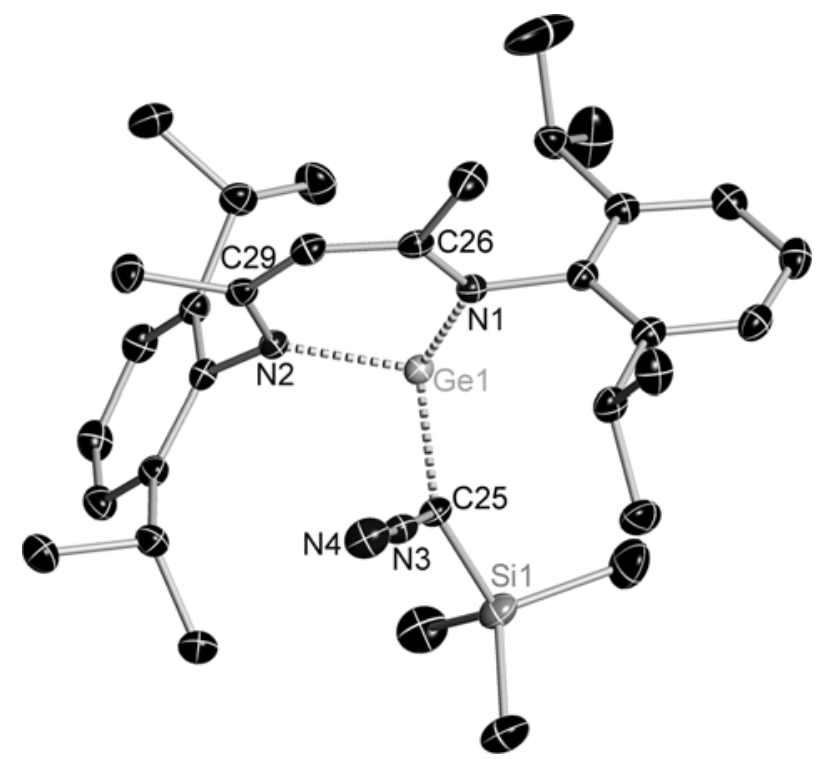

Figure 7.3. Molecular structure of 58; anisotropic displacement parameters are depicted at the $50 \%$ probability level and all restrained refined hydrogen atoms are omitted for clarity. Selected bond lengths $[\AA \AA]$ and angles $\left[{ }^{\circ}\right]$ : Ge1-C25 1.845(2), C25-Si1 1.845(2), C25-N3 1.845(2), N3-N4 1.845(2), Ge1-N1 2.030(2), Ge1-N2 2.030(2); Ge1-C25-N3 88.60(8), C25-N3-N4 88.60(8), N1-Ge1-N2 88.60(8), C25-Ge1-N1 95.52(10), C25Ge1-N2 95.55(10), N3-C25-Si1 95.55(10).

When a supernatant solution of $\mathbf{5 8}$ is stored for a longer period of time (about 3 months) at room temperature, a 1,3-silyl group as well as germanium moiety shift from the diazo carbon atom to the end-on nitrogen atom of the diazogermylene has been observed (Scheme 7.4). Thus the diazo group is converted into an isonitrile species. A comparable migration of a silyl group of azides and the lithium salt of trimethylsilyl diazomethane is known for organolanthanides. ${ }^{[246]}$ 


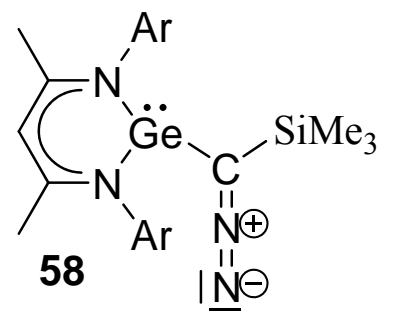

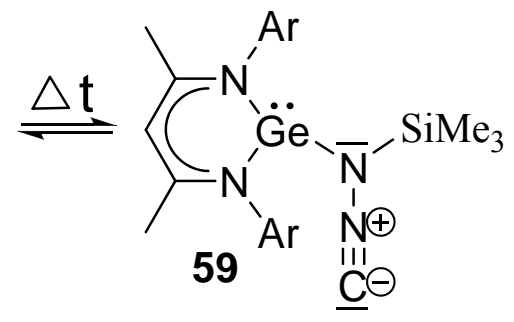

Scheme 7.4. Equilibrium between 58 and 59

The above described silyl group and germanium substituent migration has been confirmed by X-ray structure analysis of 59 (Figure 7.4). Compounds 58 and 59 are isostructural. The germanium atom in $\mathbf{5 9}$ is coordinate to three nitrogen atoms whereas the bond length of Ge1-N3 (1.9491(16) Á) is marginally shorter than the average Ge-N bond lengths of the bidentate ligand (Ge1-N1 (1.9994(16) Á, Ge1-N2 (1.9862(16) Á). The N3-Si2 (1.7699(17) Á) bond length is in the range of a standard N-Si bond (1.74 Á). The N4-C1 bond length $(1.154(3) \AA$ Á) correlates with a normal N-C triple bond $(\mathrm{N} \equiv \mathrm{C} 1.156 \AA$ ) while the N3-N4 bond $(1.350(2) \AA ̊$ ) lies between a N-N single and double bond (N-N $1.45 \AA, N=\mathrm{N}$ $1.25 \AA)$. The ${ }^{29} \mathrm{Si}$ NMR spectrum of $\mathbf{5 9}$ exhibits a singlet ( $\left.\delta 12.50 \mathrm{ppm}\right)$.

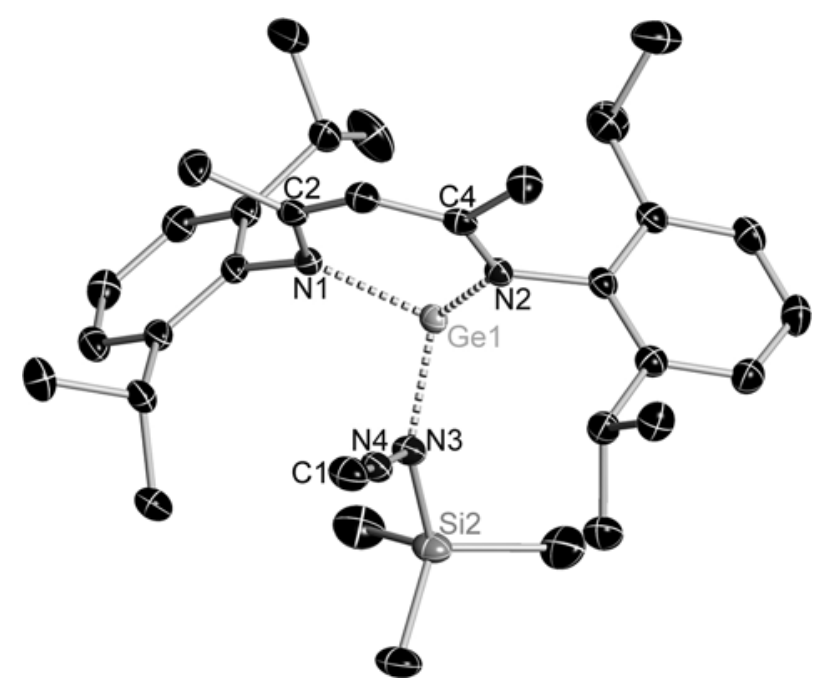

Figure 7.4. Molecular structure of 59; anisotropic displacement parameters are depicted at the $50 \%$ probability level and all restrained refined hydrogen atoms are omitted for clarity. Selected bond lengths $[\AA]$ and angles $\left[{ }^{\circ}\right]$ : Ge1-N3 1.845(2), N3-Si2 1.845(2), N3-N4 1.845(2), N4-C1 1.845(2), Ge1-N1 2.030(2), Ge1-N2 2.030(2); Ge1-N3-N4 88.60(8), N3-N4-C1 88.60(8), N1-Ge1-N2 88.60(8), N3-Ge1-N1 95.52(10), N3-Ge1-N2 95.55(10), N4-N3-Si2 95.55(10). 


\subsection{Reaction of Lithiated Trimethylsilyl Diazomethane with LSnCl}

Trialkylsilyl diazomethane derivatives are established for the synthesis of chemical building blocks $^{[247]}$ and they also possess six known or suspected structural isomers including nitrilimine and carbodiimide. In synthetic organic chemistry compounds containing the carbodiimide functionality are used as dehydration agents and to activate carboxylic acids towards amide or ester formation. For this purpose especially dicyclohexylcarbodiimide (DCC) with additives, such as N-hydroxybenzotriazole, is applied for peptide synthesis in biochemistry. ${ }^{[248-251]}$ In literature there are reports on the synthesis of carbodiimides from diazo compounds at very high temperatures or in the presence of a metal catalyst. ${ }^{[252]}$ Herein we report our efforts to exploit diazoalkane main group chemistry with $\beta$-diketiminate substituted tin(II) chloride, LSnCl. ${ }^{[146]}$

The reaction of $\mathrm{LSnCl}$ with lithiated trimethylsilyl diazomethane, obtained from $n \mathrm{BuLi}$ and $\mathrm{Me}_{3} \mathrm{SiCHN}_{2}$ in THF, resulted in the formation of $\mathrm{LSnNCNSiMe}_{3}$ (60) (Scheme 7.5). The reaction proceeds at ambient temperature without using any additional catalyst. The formation of the diazo compound $\left.\mathrm{LSnC}_{2} \mathrm{~N}_{2}\right) \mathrm{SiMe}_{3}$ (60a) or nitrilimine compound $\mathrm{LSnNNCSiMe}_{3}(\mathbf{6 0 b})$ was not observed, on the basis of analytical and structural data. In this context it is worth mentioning that the synthesis of diazo germylene and stannylene compounds was previously reported using $\mathrm{ArGeCl}\left(\mathrm{Ar}=2,6-\left(\mathrm{CH}_{2} \mathrm{NR}_{2}\right) \mathrm{C}_{6} \mathrm{H}_{3}, \mathrm{R}=\mathrm{Et}, i \mathrm{Pr}\right)$ and Ar'SnCl $\left(\mathrm{Ar}^{\prime}=2,6-\mathrm{Tip}_{2} \mathrm{C}_{6} \mathrm{H}_{3}\right.$, Tip $\left.=2,4,6-i \operatorname{Pr}_{3} \mathrm{C}_{6} \mathrm{H}_{2}\right) .{ }^{[245,253]}$ Furthermore N-bound silyl products, $\left[\left(\mathrm{MeC}_{5} \mathrm{H}_{4}\right) \mathrm{TiCl}\left(\mu-\mathrm{NSiMe}_{3}\right)\right]_{2},{ }^{[254]}$ and $\left[\left(\mathrm{C}_{5} \mathrm{Me}_{5}\right)_{2} \mathrm{Ln}\left\{\mu-\mathrm{N}\left(\mathrm{SiMe}_{3}\right) \mathrm{NC}\right\}\right]_{2}(\mathrm{Ln}=\mathrm{Sm}$, $\mathrm{La})^{[246(\mathrm{~b})]}$ have been previously observed in the reaction of $\mathrm{Li}\left[\mathrm{Me}_{3} \mathrm{SiCN}_{2}\right]$ with $\left(\mathrm{MeC}_{5} \mathrm{H}_{4}\right) \mathrm{TiCl}_{3}$ and $\left[\left(\mathrm{C}_{5} \mathrm{Me}_{5}\right)_{2} \mathrm{Ln}\right]\left[(\mu-\mathrm{Ph})_{2} \mathrm{BPh}_{2}\right](\mathrm{Ln}=\mathrm{Sm}, \mathrm{La})$ respectively with migration of the silyl group. However compound $\mathbf{6 0}$ represents the first structurally characterized carbodiimide of heavier group 14 elements containing the $\mathrm{Sn}(\mathrm{II})$ motif.

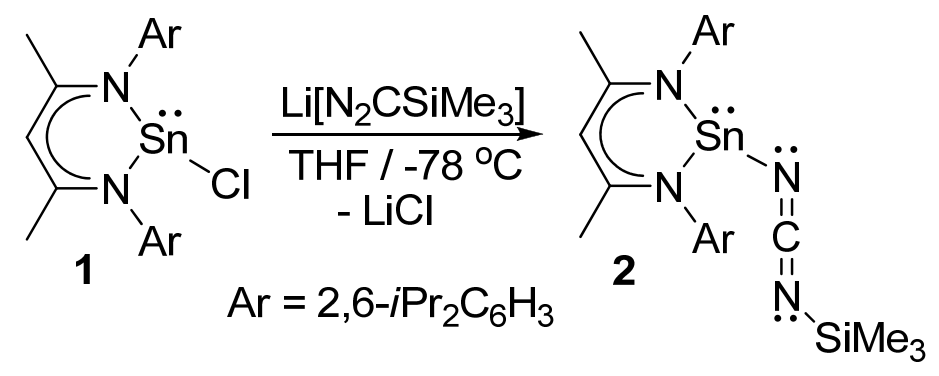


Scheme 7.5. Preparation of compound 60

It is well known that the reaction of lithium salts of substituted diazomethane with different electrophiles generates diazo or nitrilimine compounds. ${ }^{[255]}$ Therefore the present observation of a carbodiimide formation requires an unprecedented rearrangement. Wentrup and coworkers reported the evidence for nitrilimine to carbodiimide rearrangement through an isodiazirine and imidoylnitrene intermediate, based on the nature of the products obtained in the pyrolysis of different nitrilimine precursors at 500 to $960{ }^{\circ} \mathrm{C} .{ }^{[256]}$ Subsequently Bertrand and coworkers mentioned the direct evidence for the nitrilimine to imidoylnitrene rearrangement by the isolation of a unusual nitrene complex. ${ }^{[257]}$ Considering all the facts we propose the following pathway for the formation of $\mathbf{6 0}$ (Scheme 7.6).
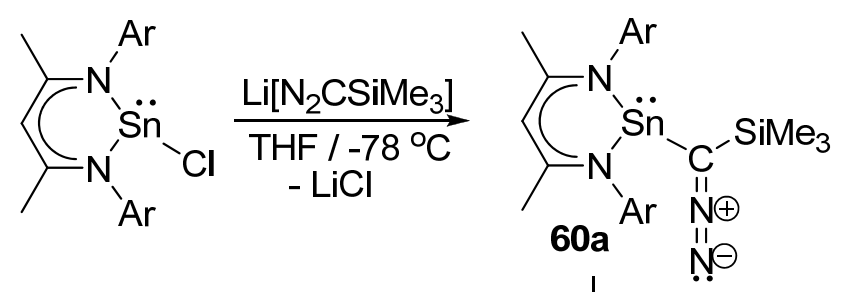

$\mathrm{Ar}=2,6-i \mathrm{Pr}_{2} \mathrm{C}_{6} \mathrm{H}_{3}$

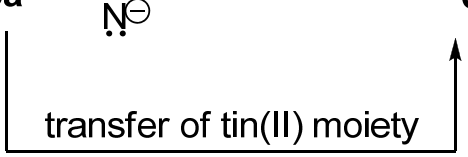

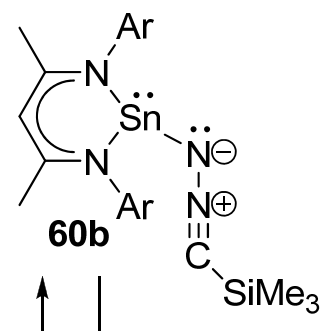
$\downarrow$ cyclisation

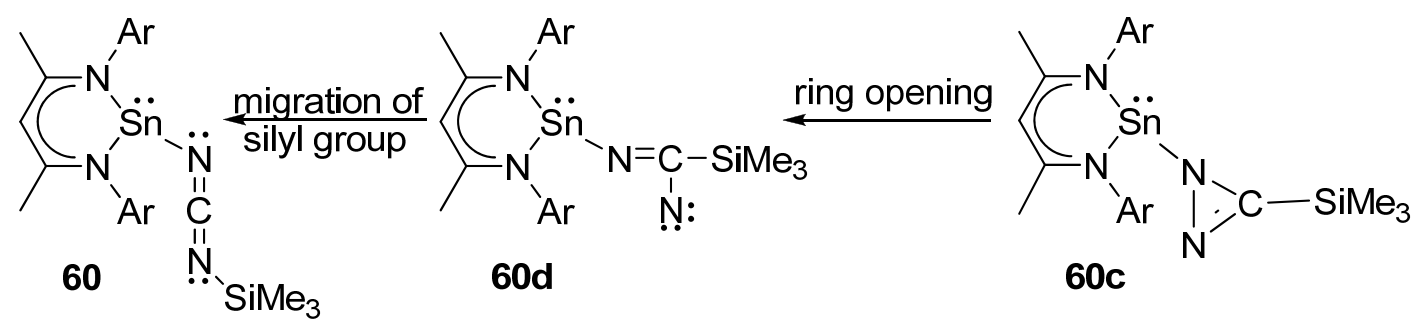

Scheme 7.6. Proposed pathway for the formation of 60

Compound 60 was characterized by multinuclear NMR spectroscopy, EI mass spectrometry, elemental analysis, and X-ray structural analysis (Figure 1). The ${ }^{1} \mathrm{H}$ NMR spectrum of $\mathbf{6 0}$ exhibits a singlet $(\delta 4.96 \mathrm{ppm})$ corresponding to the $\gamma-\mathrm{CH}$ proton. Compound $\mathbf{6 0}$ has one $\mathrm{SiMe}_{3}$ group and displays a singlet in the ${ }^{29} \mathrm{Si}$ NMR spectrum $(\delta-6.0 \mathrm{ppm})$, and shows only one type of ${ }^{13} \mathrm{C}$ satellite line due to the methyl groups with a coupling constant of $58.3 \mathrm{~Hz}$. This is an indication that the $\mathrm{SiMe}_{3}$ group binds to the $\mathrm{N}$-atom and not anymore to carbon. 
Moreover, the ${ }^{29} \mathrm{Si}$ NMR spectrum shows the resonances which are attributed to the ${ }^{15} \mathrm{~N}$ satellite with a coupling constant of $16.8 \mathrm{~Hz}$ from the nitrogen atom of the carbodiimide backbone. The ratio of the two satellite lines is well matching with their abundances. The ${ }^{119} \mathrm{Sn}$ NMR resonance of $\mathbf{6 0}$ arises at $\delta-321.4 \mathrm{ppm}$ as a triplet $\left(J\left({ }^{119} \mathrm{Sn}-{ }^{14} \mathrm{~N}\right)=250 \mathrm{~Hz}\right)$, which is upfield shifted when compared with that of the starting material $\mathrm{LSnCl}(\delta-224 \mathrm{ppm})$. In the ${ }^{14} \mathrm{~N}$ NMR spectrum the signal at $\delta-329 \mathrm{ppm}$ corresponds to the $\mathrm{N}$ atom bound to the silyl group, which was proven by the ${ }^{15} \mathrm{~N}-{ }^{1} \mathrm{H}$ correlation experiment. The resonance of the $\mathrm{NCN}$ carbon is found in the ${ }^{13} \mathrm{C}$ NMR spectrum at $\delta \quad 129.7 \mathrm{ppm}(\delta \quad 131.0 \mathrm{ppm}$; $\left.i \operatorname{Pr}_{3} \mathrm{SiNCNSnMe}_{3}\right){ }^{\left[{ }^{252]}\right.}$ In the EI mass spectrum the molecular ion is observed at $\mathrm{m} / z 649$ as the base peak for $\mathbf{6 0}$.

60 crystallizes from a saturated $n$-hexane solution at $-30{ }^{\circ} \mathrm{C}$ after two days in the monoclinic space group $C 2 / m$, with one monomer in the asymmetric unit. The coordination around the tin atom features a distorted tetrahedral geometry with one lone pair (Figure 7.5). Crystallographic studies on $\mathbf{6 0}$ also indicate that the N-bound silyl group structure $\mathrm{LSn}-\mathrm{N}=\mathrm{C}=\mathrm{N}-\mathrm{SiMe}_{3}$ was formed. All the bond lengths and bond angles are as expected. It is worth mentioning that in the solid state of $\mathbf{6 0}$ weak $\mathrm{Sn}-\mathrm{Sn}$ interactions $(3.708 \AA)$ are observed which are below the sum of van der Waals radii of $4.40 \AA$ for two tin atoms (Figure 7.6). ${ }^{[258]}$

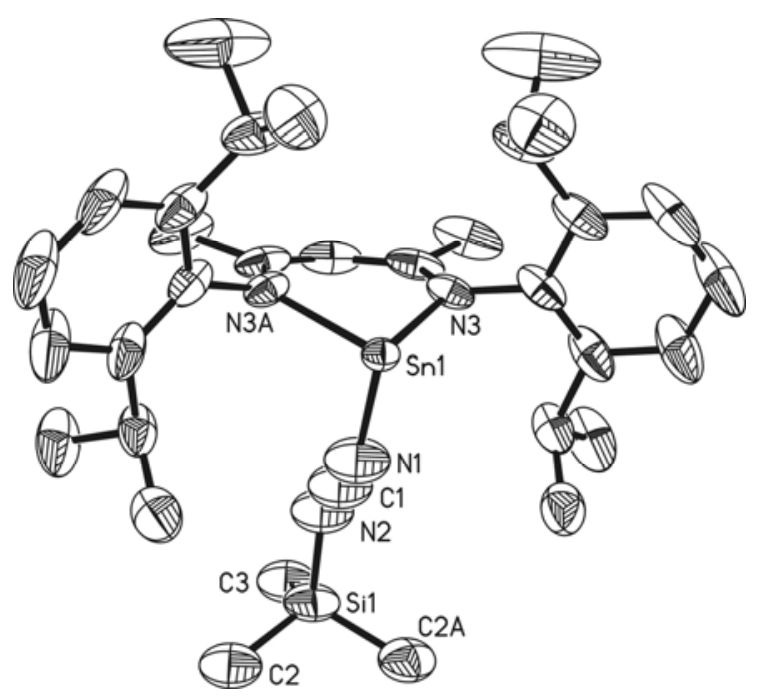

Figure 7.5. Molecular structure of 60. Thermal ellipsoids are shown at 50\% probability. $\mathrm{H}$ atoms are omitted for clarity reasons. Selected bond lengths $[\AA]$ and angles $\left[{ }^{\circ}\right]: S n 1-N 12.121(7)$, N1-C1 1.124(10), C1-N2 1.239(11), N2-Si1 1.718(7), Sn1-N3 2.169(3); Sn1-N1-C1 152.5(8), N1-C1-N2 178.6(10), C1-N2-Si1 136.2(7), N1-Sn1-N3 90.35(18), N3-Sn1-N3A 86.5(2). 


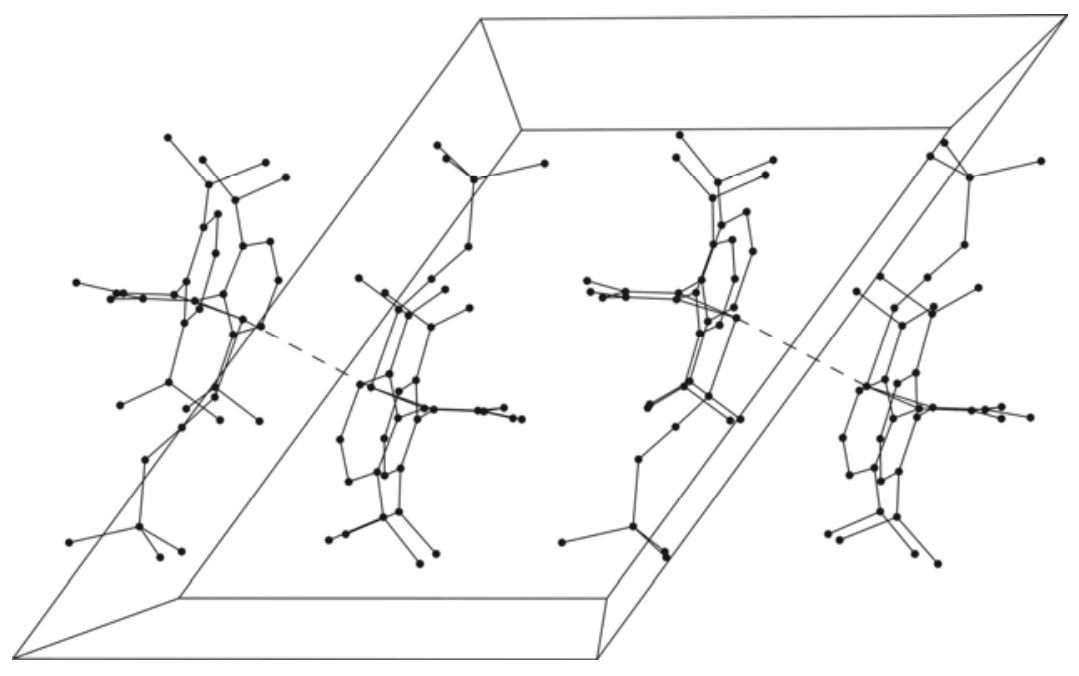

Figure 7.6. Unit cell of $\mathbf{6 0}$, showing the weak $\mathrm{Sn}-\mathrm{Sn}$ interactions (3.708 $\AA$ ) 


\section{Chapter 8}

\section{Synthesis and Reactivity of Germanium(II) and Tin(II) Compounds of the Type LMR}

After the successful synthesis of germanium(II) amide $\mathrm{LGeNH}_{2},\left(\mathrm{~L}=\mathrm{HC}(\mathrm{CMeNAr})_{2}, \mathrm{Ar}=\right.$ 2,6-i $\operatorname{Pr}_{2} \mathrm{C}_{6} \mathrm{H}_{3}$ ) (42) with the parent $\mathrm{NH}_{2}$ group, we became interested in the synthesis of another amide derivative of germanium(II). Preparation of $\mathrm{LSnNMe}_{2}$ (66) was already reported by Gibson et al. ${ }^{[259]}$ Reaction of $\mathrm{LSnNMe}_{2}$ (66) with 2-benzoylpyridine, 2,2,2trifluoro acetophenone generates the tin(II)-alkoxides $\mathrm{LSnOCPh}(2-\mathrm{Py}) \mathrm{NMe}_{2}$ (67) and $\mathrm{LSnOCPh}\left(\mathrm{CF}_{3}\right) \mathrm{NMe}_{2}(\mathbf{6 8})$ respectively by nucleophilic addition to the carbon oxygen double bond. Furthermore the reaction of $\mathbf{6 6}$ with terminal alkine $\left(\mathrm{HCCCO}_{2} \mathrm{R}, \mathrm{R}=\mathrm{Me}, \mathrm{Et}\right)$ forms tin(II)-alkylidene $\mathrm{LSnCCCO}_{2} \mathrm{R}(\mathrm{R}=\mathrm{Me},(\mathbf{6 9}) ; \mathrm{R}=\mathrm{Et}$, (70)) compounds under elimination of $\mathrm{Me}_{2} \mathrm{NH}$ rather than to undergo nucleophilic addition reaction at the carbon-carbon triple bond.

\subsection{Synthesis of $\mathrm{LGeNMe}_{2}$ and its Reaction with Elemental Sulfur}

The synthesis of $\mathrm{LGeNH}_{2}$ (42) involves the reaction of $\mathrm{L}^{\prime} \mathrm{Ge}(\mathbf{1 9})$ with $\mathrm{NH}_{3}$ gas, under the cleavage of one N-H bond. The similar reaction of $\mathrm{L}^{\prime} \mathrm{Ge}$ with $\mathrm{Me}_{2} \mathrm{NH}$ may generate the $\mathrm{LGeNMe}_{2}$. The reaction of $\mathrm{LGeCl}$ with $\mathrm{LiNMe}_{2}$ in diethyl ether afforded $\mathrm{LGeNMe}_{2}(\mathbf{6 1})$ in a good yield (Scheme 8.1). It is worth mentioning that the reaction of $\mathrm{LGeCl}$ with $\mathrm{LiN}(\mathrm{TMS})_{2}$ in diethyl ether leads exclusively to $\mathrm{L}^{\prime} \mathrm{Ge} .^{[37]}$

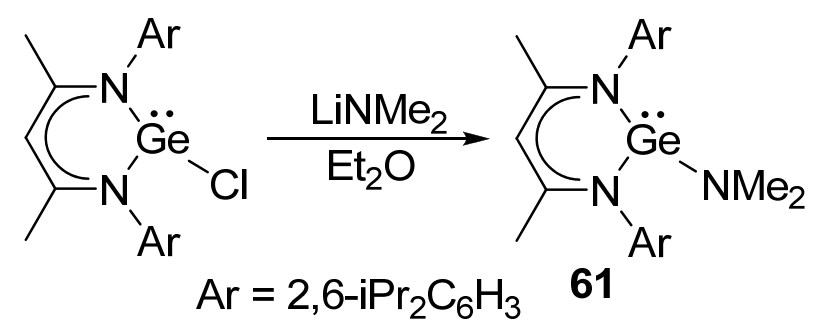

Scheme 8.1. Preparation of compound 61

61 was characterized by ${ }^{1} \mathrm{H}$ NMR spectroscopy, EI mass spectrometry, and elemental analysis. The ${ }^{1} \mathrm{H}$ NMR spectrum of $\mathbf{6 1}$ shows a singlet at $4.82 \mathrm{ppm}$ for the $\gamma-\mathrm{CH}$ proton, and two septets $(3.43,3.37 \mathrm{ppm})$ corresponding to the $\mathrm{CH}$ protons of the $i \mathrm{Pr}$ moieties. Moreover the signal arises at $\delta 2.82 \mathrm{ppm}$ corresponds to the protons of the $\mathrm{NMe_{2 }}$ group. The most abundant ion peak in the EI mass spectrum appeared at $\mathrm{m} / \mathrm{z} 491$ for the molecular ion peak 
$\left[\mathrm{M}-\mathrm{NMe}_{2}\right]^{+} .61$ crystallizes in the triclinic space group $P-1$ with one monomer in the asymmetric unit (Figure 8.1).

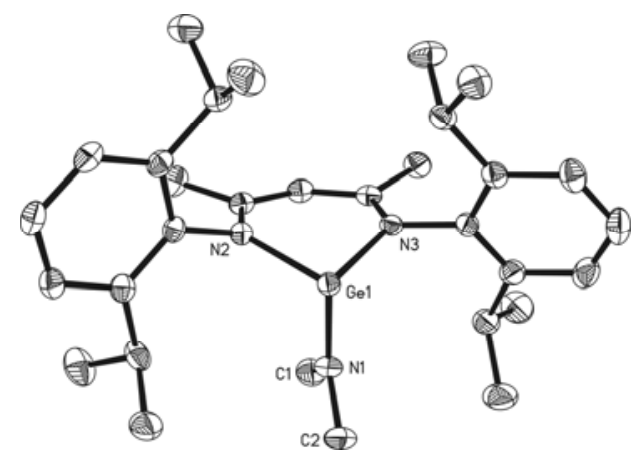

Figure 8.1. Molecular structure of $\mathbf{6 1}$. Anisotropic displacement parameters are depicted at the $50 \%$ probability level and all restrained refined hydrogen atoms are omitted for clarity. Selected bond lengths $[\AA]$ and angles $\left[{ }^{\circ}\right]$ are Ge1-N1 1.8712(14), Ge1-N2 2.0309(16); N1-Ge1-N2 99.84(7), N1-Ge1-N3 97.01(6).

We already reported on the synthesis and structure of $\mathrm{LGe}(\mathrm{S}) \mathrm{X}^{[172]}(\mathrm{X}=\mathrm{Cl}$, F) with Group 14 and 16 elements bearing a halide as well as the functionalized derivative $\mathrm{LGe}(\mathrm{S}) \mathrm{R}]$ $(\mathrm{R}=\mathrm{Me}) \cdot{ }^{[172]}$ Recently, we succeeded in the isolation and full characterization of a germanium thionoacid $\mathrm{LGe}(\mathrm{S}) \mathrm{OH}$, which has no isolated precedent in the carbon system. ${ }^{[206]}$ To the best of our knowledge there are no reports on the imide group bearing mixed unsaturated compounds containing elements of Groups 14-16. Herein we describe the formation of the germanium thionamide $\mathrm{LGe}(\mathrm{S}) \mathrm{NMe}_{2}$ (62) (Scheme 8.2). The reaction of $\mathrm{LGeNMe}_{2}$ in the presence of equivalent amounts of elemental sulfur at room temperature in toluene leads after one day to the white compound $\mathrm{LGe}(\mathrm{S}) \mathrm{NMe}_{2}(\mathbf{6 2})$ in moderate yield.

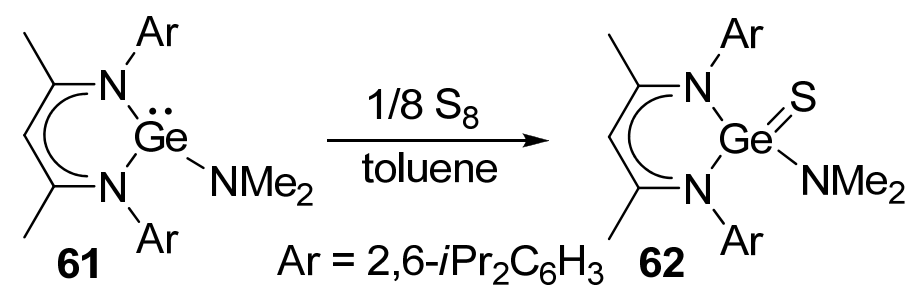

Scheme 8.2. Preparation of compound 62

Compound 62 is soluble in benzene, THF, and $n$-hexane, and shows no decomposition on exposure to dry air. Compound $\mathbf{6 2}$ was characterized by NMR spectroscopy, EI mass spectrometry. The ${ }^{1} \mathrm{H}$ NMR spectrum of $\mathbf{6 2}$ exhibits a broad resonance at $\delta 2.89-2.73 \mathrm{ppm}$ for the amide $\left(\mathrm{NMe}_{2}\right)$ hydrogens, which can be compared with singlet resonance of $\mathbf{6 1}(\delta 2.82$ ppm). The most abundant ion peak in the EI mass spectrum appeared at m/z $523\left[\mathrm{M}-\mathrm{NMe}_{2}\right]^{+}$. 


\subsection{Synthesis of LGeOTf}

LGeOTf (63) was previously reported by Driess et al. by the reaction of $\mathrm{LGeP}(\mathrm{H}) \mathrm{SiMe}_{3}$ with $\mathrm{Me}_{3} \mathrm{SiOTf}$ in the presence of $\mathrm{Et}_{3} \mathrm{~N}\left(\mathrm{OTf}=\left[\mathrm{OSO}_{2} \mathrm{CF}_{3}\right]^{-}\right) \cdot{ }^{[260]}$ Here we show the easy one pot synthetic method. LGeOTf (63) is formed under salt metathesis of $\mathrm{LGeCl}$ with AgOTf in toluene and afforded colorless compound $\mathbf{6 3}$ in high yield (Scheme 8.3).

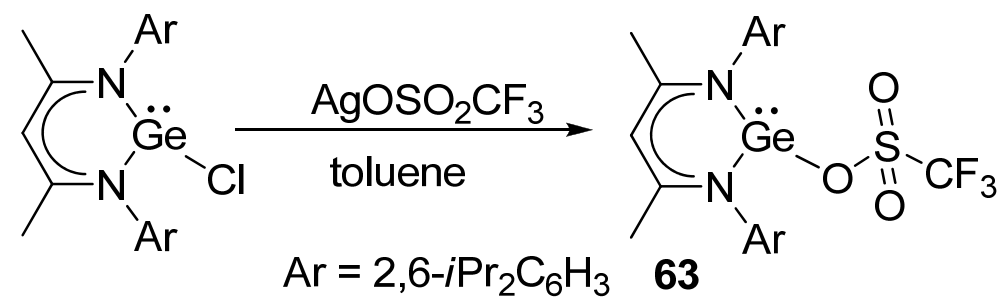

Scheme 8.3. Preparation of compound 63

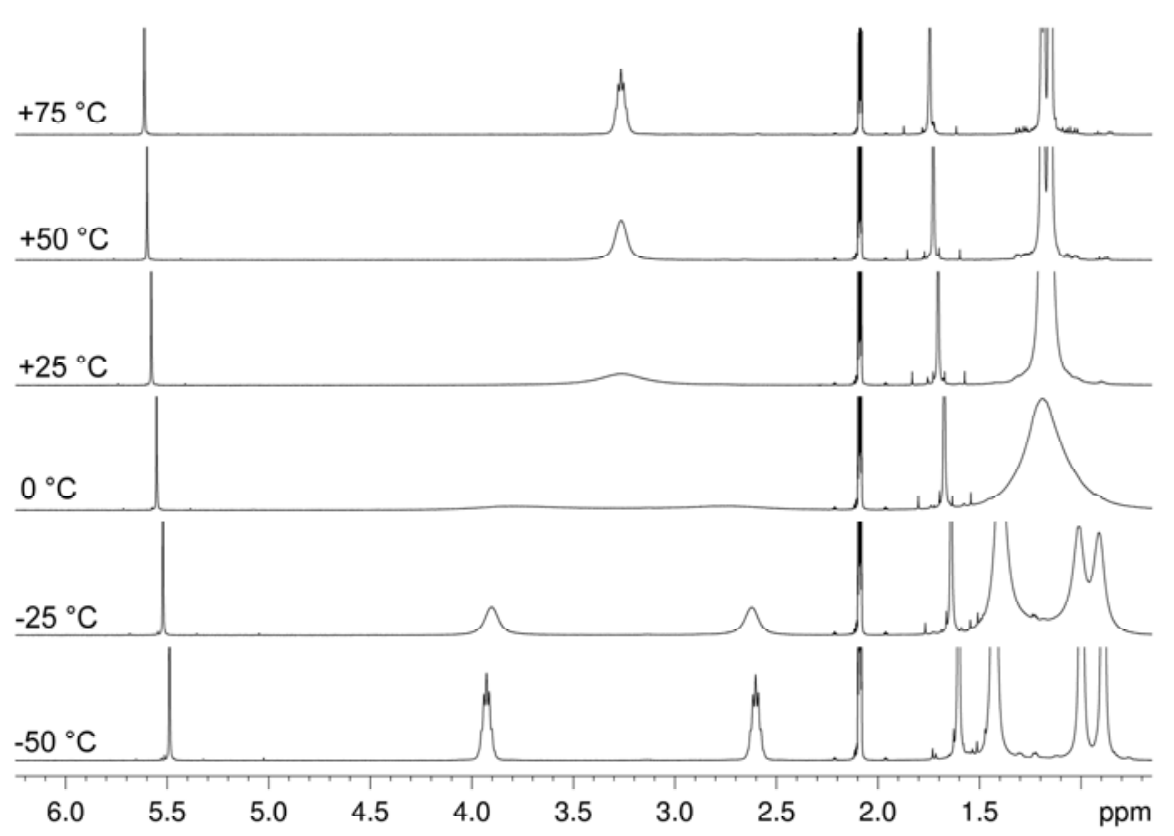

Figure 8.2. ${ }^{1} \mathrm{H}$ NMR spectrum of 63 at various temperatures

Unlike other $\beta$-diketiminate germanium compounds, the ${ }^{1} \mathrm{H}$ NMR spectrum of $\mathbf{6 3}$ shows for the $i \operatorname{Pr}$ substituents at room temperature only one broad resonance $(\mathrm{CH}, \delta 3.26 \mathrm{ppm})$ and one doublet for the methyl groups. This may be due to the rapid exchange of the triflate group at the germanium atom. Therefore we studied the temperature dependence ${ }^{1} \mathrm{H}$ NMR between +75 and $-50{ }^{\circ} \mathrm{C}$. At $-50{ }^{\circ} \mathrm{C}$ two septets appeared at 3.92 and $2.60 \mathrm{ppm}$ for the $\mathrm{CH}$ protons (Figure 8.2). However at $-50{ }^{\circ} \mathrm{C}$ the exchange of the triflate group is slow on the NMR time 
scale compared to that at room temperature. The calculated exchange rate at different temperatures is the following: $-50{ }^{\circ} \mathrm{C}, 3.5 \mathrm{sec}^{-1} ;-25{ }^{\circ} \mathrm{C}, 28 \mathrm{sec}^{-1} ; 0{ }^{\circ} \mathrm{C}, 250 \mathrm{sec}^{-1} ;+25{ }^{\circ} \mathrm{C}$, $2500 \mathrm{sec}^{-1} ;+50{ }^{\circ} \mathrm{C}, 26000 \mathrm{sec}^{-1}$.

\subsection{Synthesis of LSnMe}

Treatment of $\mathrm{LSnCl}$ with MeLi in diethyl ether at $-78{ }^{\circ} \mathrm{C}$ then allowing the solution to reach ambient temperature and keeping this for $3 \mathrm{~h}$ at this temperature afforded the corresponding methyl derivative LSnMe (64) in high yield (88\%) (Scheme 8.4). Yellow crystals of 64 suitable for single crystal X-ray analysis were obtained from a $n$-hexane solution at $-30{ }^{\circ} \mathrm{C}$ after two days. Compound 64 is thermally stable. No decomposition was observed at temperatures below its melting point $\left(170{ }^{\circ} \mathrm{C}\right)$ under an inert atmosphere. EI-MS of $\mathbf{6 4}$ gave the corresponding monomeric molecular ion peak $\mathrm{M}^{+}$. The ${ }^{1} \mathrm{H}$ NMR spectrum of 64 consists of a singlet resonance $(0.59 \mathrm{ppm})$ for $\mathrm{Sn}-\mathrm{CH}_{3}$ and is flanked by Sn satellite lines $\left({ }^{2} J\left({ }^{119} \mathrm{Sn}, \mathrm{H}\right)\right.$ $=33.07 \mathrm{~Hz}$ ). Also the ${ }^{119} \mathrm{Sn}$ NMR spectrum exhibits a singlet resonance ( $\left.\delta 192.65 \mathrm{ppm}\right)$, which is downfield shifted when compared with that of $\operatorname{LSnCl}(\delta-224 \mathrm{ppm})^{[146]}$. This change was expected because of the different electronic nature of the substituents on the tin atoms. The other resonances of the ${ }^{1} \mathrm{H}$ NMR spectrum and elemental analyses are in accordance with 64 as formulated.

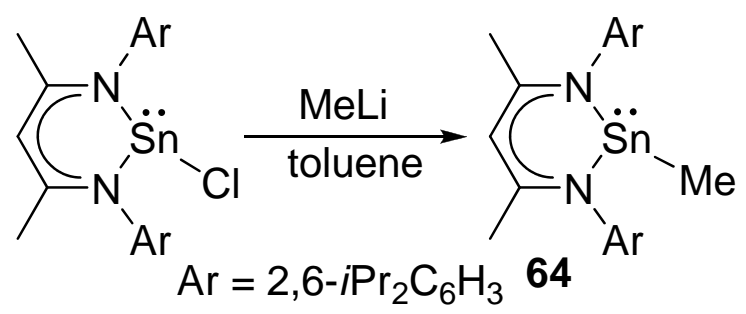

Scheme 8.4. Preparation of compound 64

The yellow compound $\mathbf{6 4}$ was also characterized by single crystal X-ray structural analysis. The molecular structure is depicted in Figure 8.3 and reveals the monomeric feature in the solid state. The $\mathrm{Sn}-\mathrm{N}$ and $\mathrm{Sn}-\mathrm{C}$ bond lengths are comparable to those found in literature. 


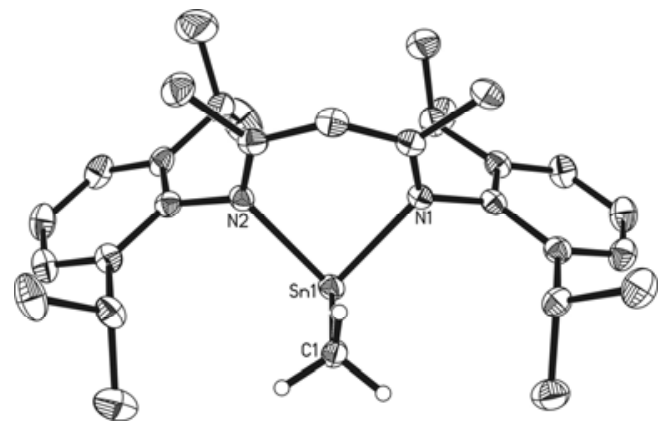

Figure 8.3. Molecular structure of 64. Selected bond lengths $[\AA]$ and angles $\left[^{\circ}\right]$; anisotropic displacement parameters are depicted at the $50 \%$ probability level and all restrained refined hydrogen atoms are omitted for clarity except Sn-Me hydrogens: Sn1-C1 2.253(2), Sn1-N1 2.2091(18), N1-C14 1.331(3); N1-Sn1-C1 92.95(8), N2-Sn1-C1 92.69(9), N1-Sn1-N2 84.69(7).

\subsection{Synthesis of Tin(II) Amides}

The preparation of the parent $\operatorname{tin}(\mathrm{II})$ amide $\mathrm{LSnNH}_{2}$ was not successful. Gibson et al. reported the synthesis of $\mathrm{LSnNMe}_{2} .{ }^{\left[{ }^{259]}\right.}$ Here we report the synthesis of $\mathrm{LSnN}\left(\mathrm{SiMe}_{3}\right)_{2}$. The welldefined compound $\mathrm{LSnN}\left(\mathrm{SiMe}_{3}\right)_{2}(\mathbf{6 5})$ was obtained in high yield from the reaction of $\mathrm{LH}$ with two equivalents of $\mathrm{KN}\left(\mathrm{SiMe}_{3}\right)_{2}$ followed by the reaction with one equivalent of $\mathrm{SnCl}_{2}$ at room temperature in THF (Scheme 8.5).

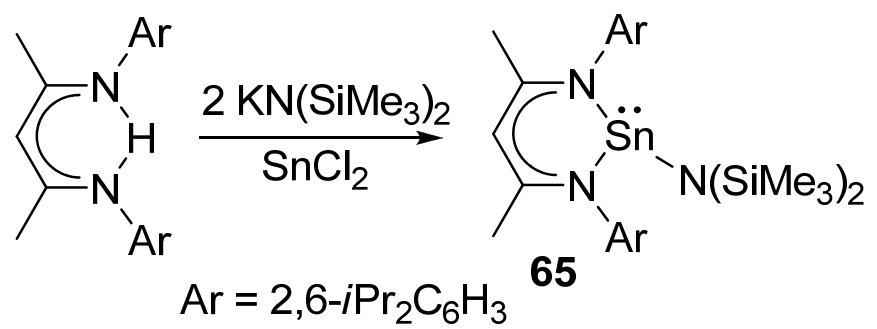

Scheme 8.5. Preparation of compound 65

Compound 65 is a yellow solid soluble in benzene, THF, $n$-hexane, and pentane and shows no decomposition on exposure to air for a short period of time. 65 was characterized by multinuclear NMR spectroscopy, EI mass spectrometry, elemental, and X-ray structural analysis. The ${ }^{1} \mathrm{H}$ NMR spectrum of compound $\mathbf{6 5}$ shows a singlet at $5.01 \mathrm{ppm}$ for the $\gamma-\mathrm{CH}$ proton, two septets (3.73 and $3.16 \mathrm{ppm}$ ) corresponding to the $\mathrm{CH}$ protons of the $i \mathrm{Pr}$ moieties and singlets at 0.46 and $0.24 \mathrm{ppm}$ for the two nonequivalent $\mathrm{SiMe}_{3}$ groups. The ${ }^{119} \mathrm{Sn} \mathrm{NMR}$ of 65 exhibits a singlet at $134.54 \mathrm{ppm}$. The most abundant ion peak in the EI mass spectrum appeared at $m / z 537$. 


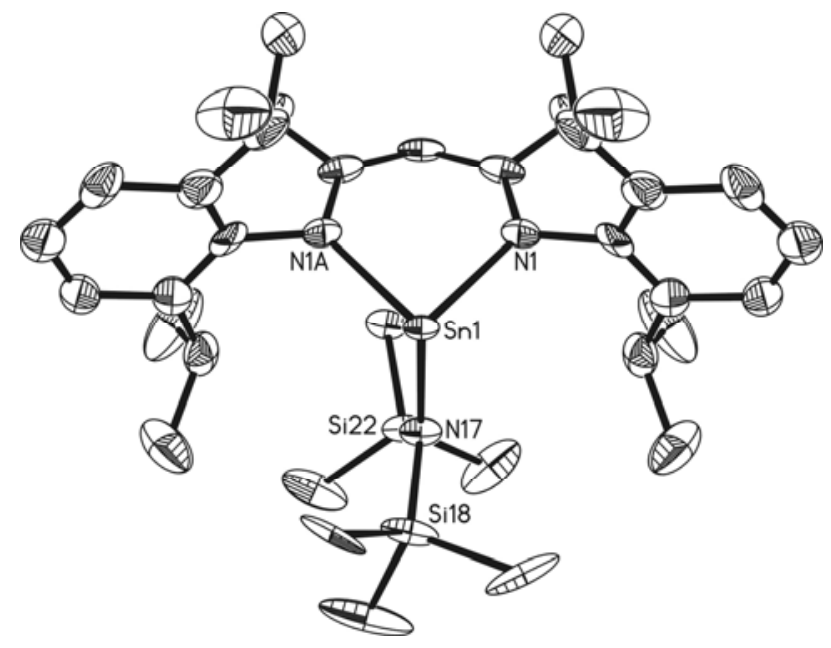

Figure 8.4. Molecular structure of 65. Anisotropic displacement parameters are depicted at the $30 \%$ probability level and all restrained refined hydrogen atoms are omitted for clarity.

Maintaining a $n$-hexane solution of $\mathbf{6 5}$ for one day at $0{ }^{\circ} \mathrm{C}$ resulted in the formation of yellow single crystals which are suitable for X-ray structural analysis. Compound $\mathbf{6 5}$ crystallizes in the orthorhombic space group $P_{n m a}$, with half a molecule in the asymmetric unit. The coordination environment of the central Sn atom exhibits a distorted tetrahedral geometry.

The di(isopropyl)phenyl moiety of the $\beta$-diketiminate ligand in $\mathbf{6 5}$ was refined with disorder at two discrete positions, applying restraints to the atomic coordinates and thermal displacement parameters (final ratio of the two positions is 50.7: 49.3). Due to disorder of the di(isopropyl)phenyl moiety of the $\beta$-diketiminate ligand for $\mathbf{6 5}$, a discussion of selected bond lengths or angles is not carried out.

We have already mentioned that $\mathrm{LSnNMe}_{2}$ has already been reported and the ${ }^{119} \mathrm{Sn}$ NMR exhibits a singlet at -172.44 ppm. ${ }^{[259]}$ Here we prepared the $\operatorname{LSnNMe}_{2}(\mathbf{6 6})$ using diethyl ether as a solvent instead of toluene and obtained 66 in an excellent yield (Scheme 8.6). The analytical data matches with previously reported values.

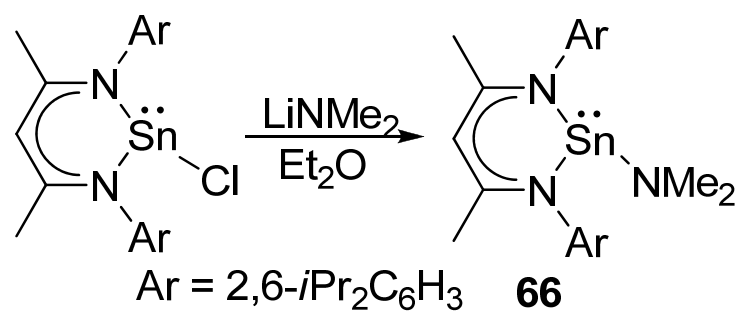

Scheme 8.6. Preparation of compound 66 


\subsection{Reaction of LSnNMe 2 with Ketones}

Lappert et al. described the addition of aminostannanes to a variety of alkines and alkenes and also aminosilylation and aminophosphination reactions with highly electrophilic substrates. ${ }^{[261]}$ Recently Hartwig et al. reported transamination of alkenes and vinylarenes by rhodium(I) amides. ${ }^{[262]}$ The insertion of an alkine into a molybdenum-amide bond has also been described. ${ }^{[263]}$ We already discussed the hydrogermylation and hydrostannylation of unsaturated compounds using $\mathrm{LGeH}$ (5) and LSnH (20) respectively. Consequently we became interested in the aminostannylation of unsaturated compounds by using $\mathrm{LSnNMe}_{2}$ (66). Although Gibson et al. reported already this compound in 2006 and studied the mechanistic properties of single-site $\beta$-diketiminate tin(II) initiators for polymerization of rac-lactide. ${ }^{[259]}$ Herein we demonstrate for the first time the aminostannylation of a carbonyl group by using tin(II) amide. Examples are the reaction of 2-benzoyl pyridine and 2,2,2trifluoro acetophenone with 66 (Scheme 8.7).

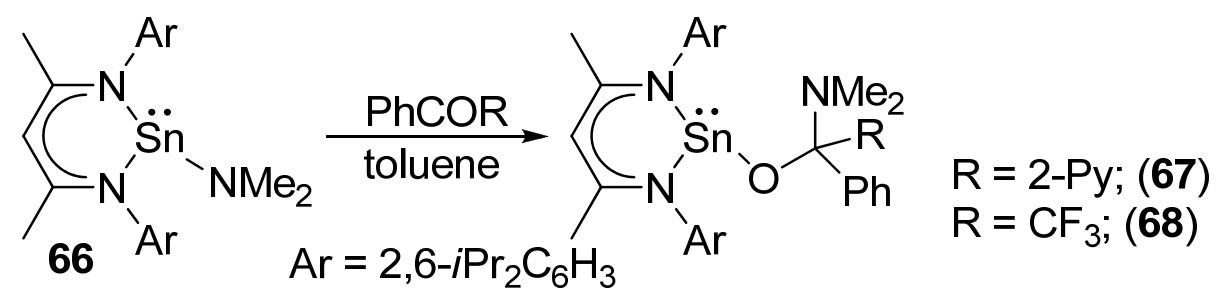

Scheme 8.7. Preparation of compounds 67 and 68

Treatment of $\mathbf{6 6}$ with 2-benzoyl pyridine and 2,2,2-trifluoro acetophenone leads almost quantitatively to the stannylene alkoxides $\mathbf{6 7}$ and 68, respectively (Scheme 8.8) with the Sn$\mathrm{O}-\mathrm{CNMe}_{2}$ core. The ${ }^{1} \mathrm{H}$ NMR spectrum of 67 exhibits a singlet ( $\delta 4.92 \mathrm{ppm}$ ) which can be assigned to the $\gamma-\mathrm{CH}$ proton and singlet resonance at $1.83 \mathrm{ppm}$ for the $\mathrm{NMe}_{2}$ group. The four isopropyl groups of $\mathbf{6 7}$ are showing four different resonances, and even the two methyl groups in the backbone exhibit two different signals in the proton NMR spectrum. The ${ }^{119} \mathrm{Sn}$ NMR resonance of 67 arises at $\delta-328$ ppm. 67 crystallizes in the triclinic space group $P-1$ with one monomer in the asymmetric unit (Figure 8.5). 


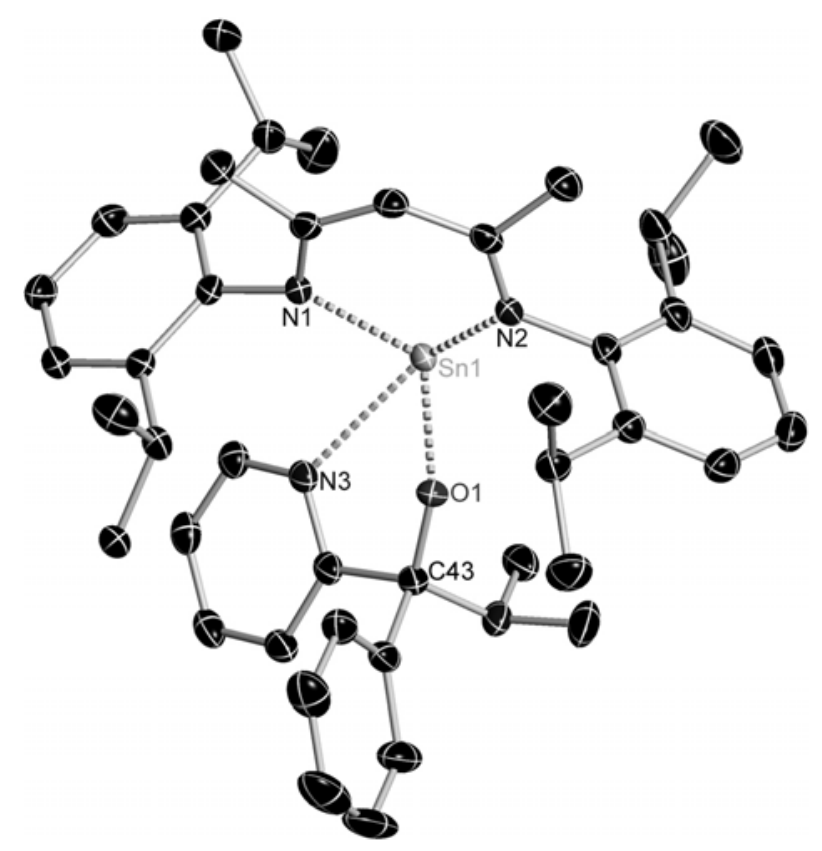

Figure 8.5. Molecular structure of 67; anisotropic displacement parameters are depicted at the $50 \%$ probability level and all restrained refined hydrogen atoms are omitted for clarity. Selected bond lengths $[\AA]$ and angles $\left[{ }^{\circ}\right]$ : Sn1-O1 2.0505(11), Sn1-N1 2.2538(13), Sn1-N3 2.5580(14), O1-C43 1.3907(19); N1-Sn1-N2 83.14(5), N1Sn1-O1 102.50(5), Sn1-O1-C43 125.03(10).

68 has one $\mathrm{CF}_{3}$ group and displays interesting NMR spectra. The ${ }^{1} \mathrm{H}$ NMR spectrum of 68 exhibits a quartet $(\delta 5.15 \mathrm{ppm})$ which corresponds to the quaternary $\mathrm{CH}$ proton. The ${ }^{19} \mathrm{~F}$ NMR resonance arises as a singlet $(\delta-69.74 \mathrm{ppm})$ and is flanked by ${ }^{119} \mathrm{Sn}$ satellite lines $\left({ }^{4} J\left({ }^{119} \mathrm{Sn},{ }^{19} \mathrm{~F}\right)=129 \mathrm{~Hz}\right)$. The ${ }^{119} \mathrm{Sn}$ NMR resonance of $\mathbf{6 8}$ arises a quartet at $\delta$-232 ppm with the same coupling constant as ${ }^{4} J\left({ }^{119} \mathrm{Sn},{ }^{19} \mathrm{~F}\right)=129 \mathrm{~Hz}$. The two ${ }^{119} \mathrm{Sn}$ NMR values of 67 and 68 are different due to the non identical electronic nature of the phenyl rings when compared with those of the $\mathrm{CF}_{3}$ group.

\subsection{Reaction of $\mathrm{LSnNMe}_{2}$ with Terminal Alkines}

Compound 66 reacts with $\mathrm{HC} \equiv \mathrm{CCO}_{2} \mathrm{Me}$ and $\mathrm{HC} \equiv \mathrm{CCO}_{2} \mathrm{Et}$ at room temperature in quantitative yield to the alkynyl linked stannylenes 69 and 70, respectively, with a $\mathrm{Sn}(\mathrm{II})-\mathrm{C} \equiv \mathrm{C}$ framework that is formed by elimination of $\mathrm{Me}_{2} \mathrm{NH}$ due to the different polarized $\mathrm{Sn}-\mathrm{NMe}_{2}$ and $\mathrm{C}-\mathrm{H}$ bonds (Scheme 8.8). 69 and 70 were monitored by their ${ }^{1} \mathrm{H}$ NMR spectra. Sharp resonances in the ${ }^{1} \mathrm{H}$ NMR of 69 and 70 indicated that the products have been formed in high yield. In the course of reaction there is no sign that some side products are formed by nucleophilic addition of $\mathrm{LSnNMe}_{2}$ to the carbon carbon triple bond, which we observed exclusively in the case of LSnH. 


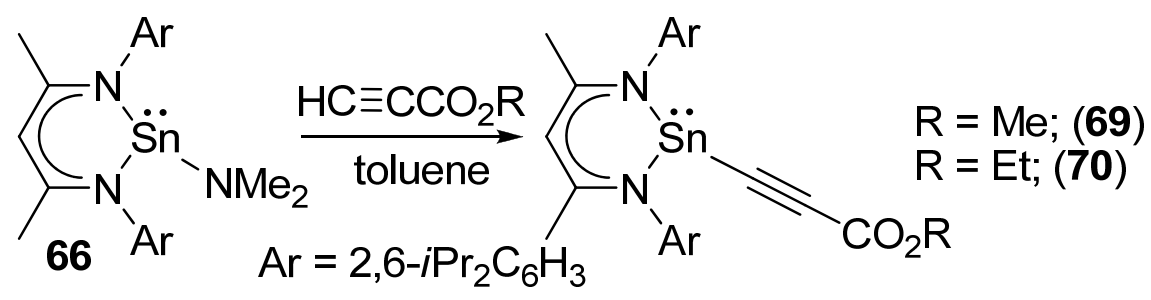

Scheme 8.8. Preparation of compounds 69 and 70

In the ${ }^{1} \mathrm{H}$ NMR spectra of $\mathbf{6 9}$ and 70, the complete disappearance of the proton resonance of $\mathrm{Sn}-\mathrm{NMe}_{2}$ was observed. This indicates the complete conversion of the tin amide to the corresponding tin-alkenyl compounds.

69 and 70 are yellow solids soluble in benzene, THF, $n$-hexane, and $n$-pentane, respectively and show no decomposition on exposure to air. 69 and 70 were characterized by multinuclear NMR and IR spectroscopy, EI mass spectrometry, and elemental analysis. Furthermore 70 was characterized by X-ray structural analysis (Figure 8.6).

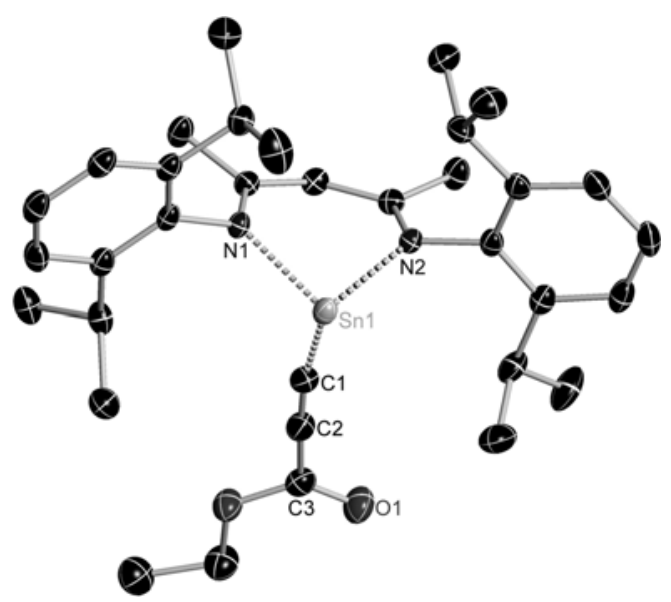

Figure 8.6. Molecular structure of 70; anisotropic displacement parameters are depicted at the $50 \%$ probability level and all restrained refined hydrogen atoms are omitted for clarity. Selected bond lengths $[\AA]$ and angles $\left[^{\circ}\right]$ : Sn1-N1 2.1802(16), Sn1-C1 2.214(2), C1-C2 1.213(3); C2-C3 1.447(3), C3-O1 1.208(3), C3-O2 1.343(3); N1-Sn1-N2 86.33(6), N1-Sn1-C1 89.90(7), Sn1-C1-C2 168.83(19), C1-C2-C3 176.9(2).

The ${ }^{1} \mathrm{H}$ NMR spectrum of 70 shows a quartet and a triplet resonance ( $\delta 3.92$ and $\left.0.92 \mathrm{ppm}\right)$ corresponding to the two different types of $\mathrm{CH}$ protons of the ethyl moieties. The ${ }^{119} \mathrm{Sn}$ NMR resonance of 69 and 70 arises at $\delta$-254.91 and $-252.97 \mathrm{ppm}$, which are very close to each 
other. In Table 8.1 we have summarised all the ${ }^{119} \mathrm{Sn}$ NMR data of related compounds including $\mathrm{LSnNMe}_{2}$ (66). Compound 70 crystallizes in the monoclinic space group $P 2_{1} / \mathrm{n}$, with one monomer in the asymmetric unit, and with four molecules in each unit cell. Single crystals were obtained after two days from a saturated $n$-hexane solution at $-32{ }^{\circ} \mathrm{C}$ in a freezer. The coordination polyhedron around the tin atom features a distorted tetrahedral geometry with a stereochemically active lone pair.

Table 8.1. ${ }^{119} \mathrm{Sn}$ NMR data of Sn(II) compounds

\begin{tabular}{|c|c|}
\hline Compound $^{*}$ & ${ }^{119} \mathrm{Sn}\left\{{ }^{1} \mathrm{H}\right\} \operatorname{NMR}(\delta, \mathrm{ppm})$ \\
\hline LSnMe (64) & 192.65 \\
\hline $\mathrm{LSnN}\left(\mathrm{SiMe}_{3}\right)_{2}(\mathbf{6 5})$ & -134.54 \\
\hline $\mathrm{LSnNMe}_{2}(\mathbf{6 6})$ & -172.44 \\
\hline $\mathrm{LSnOCPh}\left(\mathrm{NMe}_{2}\right)(2-\mathrm{Py})(67)$ & -328 \\
\hline $\mathrm{LSnOCPh}\left(\mathrm{NMe}_{2}\right)\left(\mathrm{CF}_{3}\right)(\mathbf{6 8})$ & -232 \\
\hline $\mathrm{LSnCCCO}_{2} \mathrm{Me}(\mathbf{6 9})$ & -254.91 \\
\hline $\mathrm{LSnCCCO}_{2} \mathrm{Et}(\mathbf{7 0})$ & -252.97 \\
\hline
\end{tabular}

$* \mathrm{~L}=\mathrm{HC}(\mathrm{CMeNAr})_{2} ; \mathrm{Ar}=2,6-i \mathrm{Pr}_{2} \mathrm{C}_{6} \mathrm{H}_{3}$ 


\section{Chapter 9}

\section{Synthesis of a Monomeric Tin(II) Hydroxide}

The synthesis of tin(II) hydroxide has a long standing interest especially using aqueous routes. In the course of this approach no successful preparation of tin(II) hydroxide was reported but a number of tin(II) oxy-hydroxide species with varying ratios of tin, oxygen, and hydrogen have been prepared. In 1968 Howie and Moser were able to present a compound of composition $3 \mathrm{SnO} \cdot \mathrm{H}_{2} \mathrm{O}$ which was structurally characterised as $\mathrm{Sn}_{6} \mathrm{O}_{4}(\mathrm{OH})_{4} \cdot{ }^{\left[{ }^{[264]}\right.}$ The eight oxygen atoms are forming a regular cube joined by hydrogen bonds and superimposed by an octahedron of tin atoms. The structural unit is related to $\mathrm{Sn}_{3}(\mathrm{OH})_{4}{ }^{2+}$, which was demonstrated to exist in solution by Tobias et al. ${ }^{[265]}$ and it can be derived by a condensation-deprotonation reaction. Finally in 1995 the cationic structure was determined by Donaldson et al. ${ }^{[266]}$ Therefore, it is not surprising that all attempts to isolate tin(II) hydroxides have been unsuccessful till to date via an aqueous route. Additionally a tin(II) hydroxide has been synthesized by an anhydrous organometallic method involving the exchange of substituents between a triorganotin hydroxide and tin(II) dichloride. ${ }^{[267]}$ The resulting product was only characterised by Mössbauer and IR spectroscopy and elemental analysis. In 2005 Power and coworkers reported on a structurally characterised dimeric tin(II) hydroxide stabilised by a bulky aryl ligand. ${ }^{[132]}$ This hydroxide was prepared from $\mathrm{Ar}^{\prime} \mathrm{SnSnAr} \mathrm{Ar}^{\prime}\left(\mathrm{Ar}^{\prime}=\mathrm{C}_{6} \mathrm{H}_{3}-2,6\left(\mathrm{C}_{6} \mathrm{H}_{3}-\right.\right.$ $\left.i \mathrm{Pr}_{2}\right)_{2}$ ) and 2,2,6,6-tetramethylpiperidine-N-oxide (TEMPO) or nitrous oxide $\left(\mathrm{N}_{2} \mathrm{O}\right)$ by serendipity. Moreover, only a few examples of well-characterized tin(IV) hydroxides are known in literature. ${ }^{[131]}$ To the best of our knowledge there is presently no report available on a well-designed synthesis and structural characterization of any monomeric tin(II) hydroxide.

\subsection{Synthesis of $\mathrm{LSnCIFe}(\mathrm{CO})_{4}$}

The reaction of $\mathrm{LSnCl}$ with diiron nonacarbonyl, $\mathrm{Fe}_{2}(\mathrm{CO})_{9}$ in $\mathrm{THF}$ leads to $\mathrm{LSnClFe}(\mathrm{CO})_{4}$ (71) (Scheme 9.1). Compound 71 is insoluble in $n$-hexane or $n$-pentane, but soluble in the benzene, toluene, diethyl ether, and THF. 


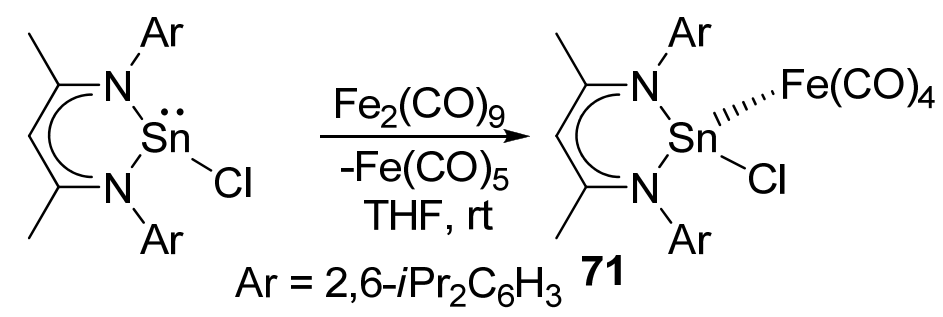

Scheme 9.1. Preparation of compound 71

The ${ }^{119} \mathrm{Sn}$ NMR spectrum exhibits a singlet resonance $(\delta 88.98 \mathrm{ppm})$. This is downfield shifted compared with that of the starting precursor $\operatorname{LSnCl}(\delta-224 \mathrm{ppm}),{ }^{[146]}$ because of the change of coordination number around the tin center. Moreover, carbonyl absorptions for 71 were found at $\tilde{v}=2043 \mathrm{~s}, 1968 \mathrm{~s}, 1939 \mathrm{~s} \mathrm{~cm}^{-1}$, these are in the expected range for terminal carbonyl groups.

The molecular structure of $\mathbf{7 1}$ has been determined by single crystal X-ray diffraction analysis (Figure 9.1) which demonstrates that the complex exists as a monomer. Colorless compound 71 crystallizes in the monoclinic space group $P 2_{1} / n$, with one monomer in the asymmetric unit from saturated toluene solution at $-32{ }^{\circ} \mathrm{C}$ after four days. The coordination polyhedron around the tin atom features a distorted tetrahedral geometry with unique $\mathrm{Cl}$ group (Figure 9.1).

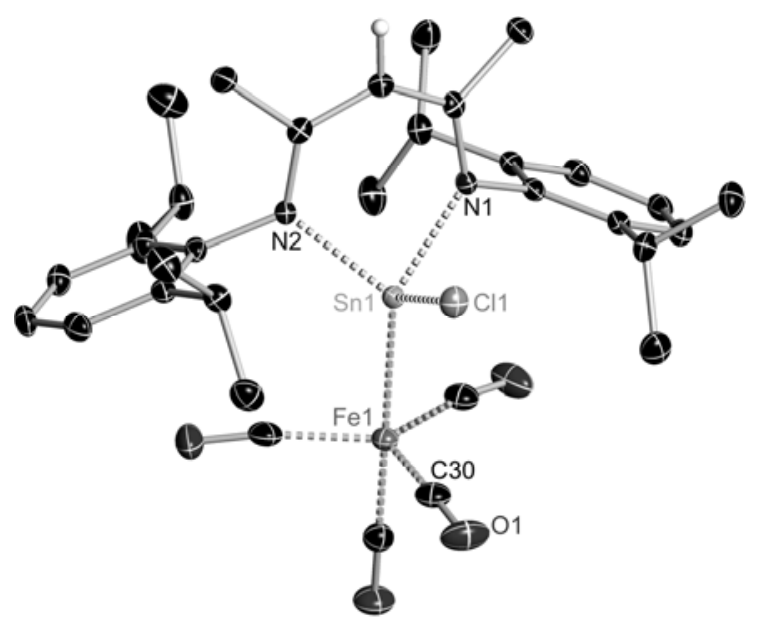

Figure 9.1. Molecular structure of 71. Anisotropic displacement parameters are depicted at the $50 \%$ probability level and all restrained refined hydrogen atoms are omitted for clarity. Selected bond lengths $[\AA]$ and angles $\left[^{\circ}\right]$ : Sn1-C11 2.3893(6), Sn1-N1 2.1132(16), Sn1-Fe1 2.4823(4), Fe1-C30 1.793(2), C30-O1 1.148(3); N1-Sn1C11 94.94(5), N1-Sn1-N2 88.90(6), Cl1-Sn1-Fe1 118.200(15), Fe1-C30-O1 175.75(19).। 


\subsection{Synthesis of $\mathrm{LSn}\left(\mathrm{NMe}_{2}\right) \mathrm{Fe}(\mathrm{CO})_{4}$}

The reaction of $\mathrm{LSnNMe}_{2}(\mathbf{6 6})$ in the presence of one equivalent of $\mathrm{Fe}_{2}(\mathrm{CO})_{9}$ in THF leads after $24 \mathrm{~h}$ to the pale-orange compound $\mathrm{LSn}\left(\mathrm{NMe}_{2}\right) \mathrm{Fe}(\mathrm{CO})_{4}(\mathbf{7 2})$ in good yield $(85 \%)$ (Scheme 9.2). Compound 72 is soluble in benzene, THF, and diethyl ether and shows no decomposition on exposure to dry air. The ${ }^{1} \mathrm{H}$ NMR spectrum of 72 shows a broad resonance at (2.8-3.1 ppm) which can be assigned to the $\mathrm{NMe}_{2}$ protons.

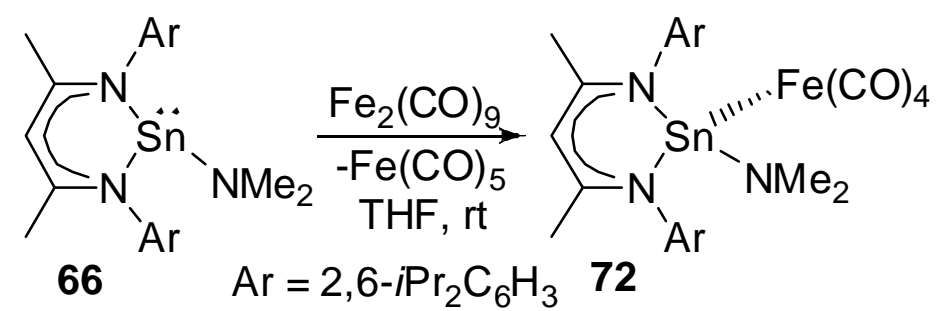

Scheme 9.2. Preparation of compound 72

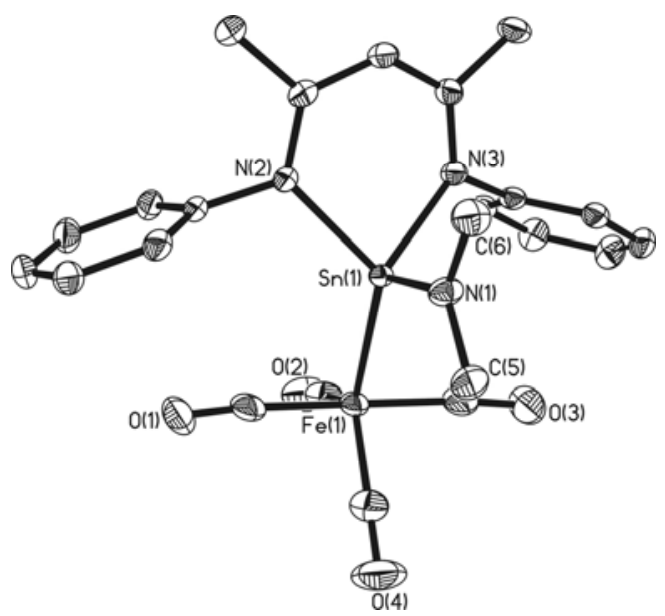

Figure 9.2. Molecular structure of $\mathbf{7 2 \cdot 0 . 5 T H F}$. Thermal elipsoids are shown at $50 \%$ probability. One of the two molecules of 72, solvent THF, isopropyl groups and $\mathrm{H}$ atoms are omitted for clarity reasons. Selected bond lengths $[\AA]$ and angles $\left[^{\circ}\right]$ : Sn1-N1 2.014(3), Sn1-Fe1 2.4832(7), Sn1-N2 2.151(2), Fe1-C1 1.794(4), C1-O1 1.142(4); N1-Sn1-N2 100.70(10), N2-Sn1-N3 86.36(9), N2-Sn1-Fe1 125.80(7).

Maintaining a THF solution of $\mathbf{7 2}$ for two days at $-32{ }^{\circ} \mathrm{C}$ resulted in pale-orange single crystals suitable for X-ray structural analysis. Compound $\mathbf{7 2}$ crystallizes in the monoclinic 
space group $P 2_{1} / c$, with two molecules of 72 and one THF molecule in the asymmetric unit. Complex 72 exists as a monomer in the solid state (Figure 9.2). The coordination polyhedron around the tin atom comprises two nitrogen atoms from the supporting ligand, one nitrogen atom from $\mathrm{NMe}_{2}$ group, and one iron atom from the coordinated $\mathrm{Fe}(\mathrm{CO})_{4}$ in a distorted tetrahedral geometry.

\subsection{Synthesis of a Monomeric Tin(II) Hydroxide}

Based on our experience in the synthesis of $\mathrm{LGeOH}(15),{ }^{[127]}$ we reacted $\mathrm{LSnCl}^{[146]}(\mathrm{L}=$ $\left.\mathrm{HC}(\mathrm{CMeNAr})_{2} ; \mathrm{Ar}=2,6-i \mathrm{Pr}_{2} \mathrm{C}_{6} \mathrm{H}_{3}\right)$ with water in the presence of a $\mathrm{N}$-heterocyclic carbene in a broad temperature range $\left(-78{ }^{\circ} \mathrm{C}\right.$ to $\left.\mathrm{rt}\right)$ in coordinating as well as in non coordinating solvents. From the reaction mixture we obtained the protonated ligand (LH) and a hydrocarbon insoluble white precipitate. This insoluble part mainly contained the Nheterocyclic carbene $\mathrm{HCl}$ adduct and tin hydroxy species. Also the reaction of $\operatorname{LSnNR}_{2}(\mathrm{R}=$ $\mathrm{Me}(\mathbf{6 6})$ and $\mathrm{SiMe}_{3}(\mathbf{6 5})$ ) with equivalent amounts of water leads preferentially to $\mathrm{LH}$ elimination. Finally under the protection with a metal fragment we shielded the tin(II) center to avoid protonation at the tin.

The slow addition of an equivalent amount of degassed water to a THF solution of 72 at -60 ${ }^{\circ} \mathrm{C}$ resulted in the formation of the orange compound $\mathrm{LSn}(\mathrm{OH}) \mathrm{Fe}(\mathrm{CO})_{4}(\mathbf{7 3})$ in good yield (73 $\%$ ) under elimination of volatile $\mathrm{Me}_{2} \mathrm{NH}$ (Scheme 9.3).

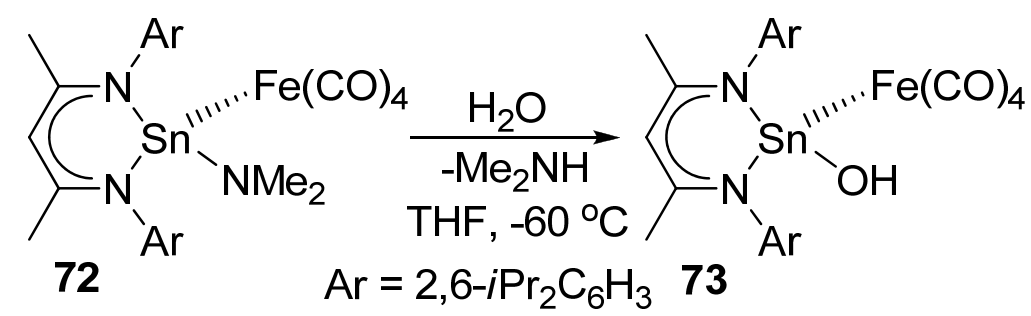

Scheme 9.3. Preparation of compound 73

Compound $\mathbf{7 3}$ is a brown solid soluble in benzene, THF, and diethyl ether and insoluble in $n$ hexane, and $n$-pentane, and it is showing remarkable stability to dry air. $\mathbf{7 3}$ was characterized by ${ }^{1} \mathrm{H},{ }^{119} \mathrm{Sn} \mathrm{NMR}$, and IR spectroscopy, EI mass spectrometry, elemental analysis and X-ray structural analysis. In the ${ }^{1} \mathrm{H}$ NMR spectrum of $\mathbf{7 3}$, the $\mathrm{Sn}-\mathrm{OH}$ proton resonance was detected ( $\delta 1.66 \mathrm{ppm})$, and the complete disappearance of the proton resonance of $\mathrm{Sn}\left(\mathrm{NMe}_{2}\right)$ was 
observed. This indicates the conversion of the tin amide to the corresponding tin hydroxide. Moreover the ${ }^{119} \mathrm{Sn}$ NMR for $\mathbf{7 3}$ shows a resonance at $\delta 45 \mathrm{ppm}$ which is shifted upfild when compared with that of $\mathbf{7 2}(\delta 94 \mathrm{ppm})$. The EI mass spectrum of $\mathbf{7 3}$ exhibits the molecular ion peak at $\mathrm{m} / \mathrm{z} 722$ albeit in low intensity. The base peak for 73 was found at $\mathrm{m} / \mathrm{z} 705$ corresponding to $[\mathrm{M}-\mathrm{OH}]^{+}$. The IR spectrum of 73 displays a band at $3615 \mathrm{~cm}^{-1}$, a plausible range in light of the $3390 \mathrm{~cm}^{-1}$ band measured for $\mathrm{Sn}(\mathrm{OH})_{2}{ }^{[267]}$ and $3480 \mathrm{~cm}^{-1}$ for $\left(\mathrm{Ar}^{\prime} \mathrm{SnOH}\right)_{2}\left(\mathrm{Ar}^{\prime}=2,6-\left(2,6-i \mathrm{Pr}_{2} \mathrm{C}_{6} \mathrm{H}_{3}\right)_{2} \mathrm{C}_{6} \mathrm{H}_{3}\right)^{[132]}$ and $3599 \mathrm{~cm}^{-1}$ for $\mathrm{LGe}(\mathrm{OH}) \mathrm{Fe}(\mathrm{CO})_{4} \cdot{ }^{[268]} \mathrm{In}$ the IR spectrum of $\mathrm{LSn}(\mathrm{OD}) \mathrm{Fe}(\mathrm{CO})_{4}$, which we have prepared accordingly from 72 and $\mathrm{D}_{2} \mathrm{O}$, no band is observed at $3615 \mathrm{~cm}^{-1}$. The measured $\mathrm{O}-\mathrm{D}$ wave number of $2690 \mathrm{~cm}^{-1}$ is in the range of the calculated one $\left(2631 \mathrm{~cm}^{-1}\right)$.

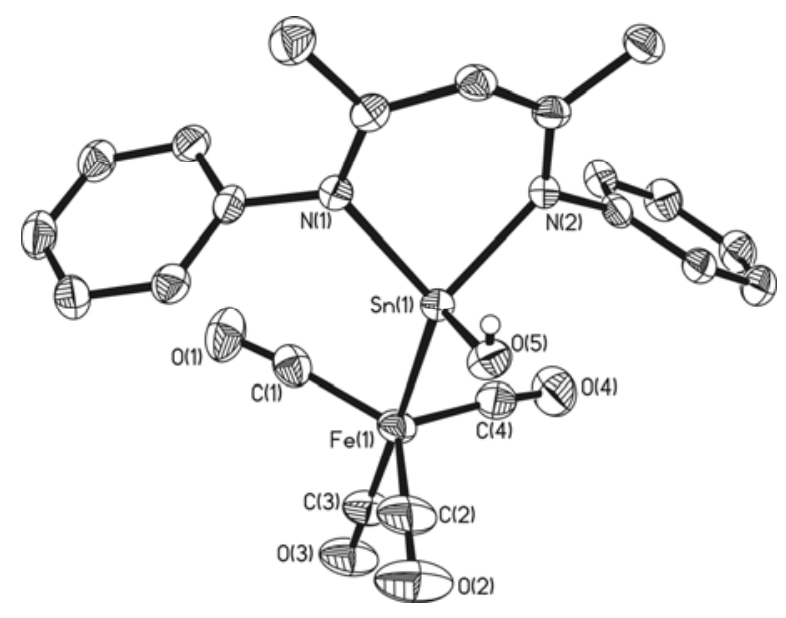

Figure 9.3. Molecular structure of 73. Thermal elipsoids are shown at 50\% probability. Isopropyl groups and $\mathrm{H}$ atoms except at $\mathrm{O} 5$ are omitted for clarity reasons. Selected bond lengths $[\AA]$ and angles $\left[{ }^{\circ}\right]$ : Sn1-O5 2.001(3), Sn1-Fe1 2.4719(7), Sn1-N1 2.130(3), Fe1-C1 1.796(4), C1-O1 1.146(5); N1-Sn1-N2 88.41(10), N1-Sn1-O5 97.26(12), N1-Sn1-Fe1 125.31(7).

Compound 73 crystallizes in the monoclinic space group $P 2_{1} / n$, with one monomer in the asymmetric unit from a saturated toluene solution at $-32{ }^{\circ} \mathrm{C}$ after four days. The coordination polyhedron around the tin atom features a distorted tetrahedral geometry with unique $\mathrm{OH}$ group (Figure 9.3). More interestingly the $\mathrm{OH}$ group is not involved in any kind of hydrogen bonding which is reflected in the IR spectrum. Its structure is very similar to that of its germanium analogue $\mathrm{LGe}(\mathrm{OH}) \mathrm{Fe}(\mathrm{CO})_{4}{ }^{[268]}$ In the structures of $\mathbf{7 2}$ and $\mathbf{7 3}$ the $\mathrm{Sn}-\mathrm{N}$ bond lengths are 2.151(2), 2.130(3) $\AA$ and N-Sn-N bond angles are 86.36(9), 88.41(10 ${ }^{\circ}$, respectively ( $\mathrm{N}$ are the nitrogen atoms of the ligand backbone). Comparing this data with the slightly longer Sn-N bond lengths and smaller N-Sn-N angles in LSnF (1) and LSnMe (64) 
(2.178(3), 2.2091(18) $\AA$ and 86.22(10), 84.69(7) $\left.{ }^{\circ}\right)$ illustrates the influence of the different coordination numbers around the tin atom. A noteworthy feature of compound $\mathbf{7 3}$ is the $\mathrm{SnOH}$ moiety. The crystallographic O-H distance $(0.60 \AA)$ is very short but the hydrogen was found and refined without any restraints. The Sn-O distance (2.001(3) $\AA$ ) is indicative for a Sn-O single bond and the Sn-O-H angle $\left(109.58(2)^{\circ}\right)$ is as expected. Due to the absence of any involvement in hydrogen bonding the Sn-O bond length is slightly shorter than those in the dimeric structure $\left(\mathrm{Ar}^{\prime} \mathrm{SnOH}\right)_{2}(\mathrm{av} 2.147 \AA)\left(\mathrm{Ar}^{\prime}=2,6-\left(2,6-i \mathrm{Pr}_{2} \mathrm{C}_{6} \mathrm{H}_{3}\right)_{2} \mathrm{C}_{6} \mathrm{H}_{3}\right)^{[132]}$ and the values predicted for $\mathrm{Sn}_{3}(\mathrm{OH})_{4}{ }^{2+}$ (av $2.217 \AA$ ). ${ }^{[266]}$ Interestingly, and as might be expected, shorter Sn-OH bond lengths are found in tin(IV) compounds due to the smaller radius of $\mathrm{Sn}(\mathrm{IV})$ compared to that of $\mathrm{Sn}(\mathrm{II}) \quad$ (e.g. Sn-O 1.968(4) $\AA$ for $\mathrm{Sn}-\mathrm{OH}$, in $\left[\left(\mathrm{Me}_{3} \mathrm{Si}\right)_{3} \mathrm{CSnO}(\mathrm{OH})\right]_{3} .{ }^{[115]}$

Table 9.1. Comparison of ${ }^{119} \mathrm{Sn}$ NMR data of $\mathrm{Sn}(\mathrm{II})$ Compounds with and without Coordination to an Iron-Carbonyl Fragment.

\begin{tabular}{|c|c|}
\hline Compound $^{*}$ & ${ }^{119} \mathrm{Sn} \mathrm{NMR}(\delta \mathrm{ppm})$ \\
\hline $\mathrm{LSnCl}$ & -224 \\
\hline $\mathrm{LSnNMe}_{2}(\mathbf{6 6})$ & -172 \\
\hline $\mathrm{LSnClFe} \mathrm{CO})_{4}(\mathbf{7 1})$ & 88.98 \\
\hline $\mathrm{LSn}\left(\mathrm{NMe}_{2}\right) \mathrm{Fe}(\mathrm{CO})_{4}(\mathbf{7 2})$ & 94.00 \\
\hline $\mathrm{LSn}(\mathrm{OH}) \mathrm{Fe}(\mathrm{CO})_{4}(\mathbf{7 3})$ & 45.03 \\
\hline
\end{tabular}

$* \mathrm{~L}=\mathrm{HC}(\mathrm{CMeNAr})_{2} ; \mathrm{Ar}=2,6-i \mathrm{Pr}_{2} \mathrm{C}_{6} \mathrm{H}_{3}$ 


\section{Chapter 10}

\section{Summary}

In this thesis unprecedented synthetic and reactivity studies of the compounds with low valent group 14 elements are reported. We have demonstrated the reactivity of the first isolable three coordinate germanium(II) and tin(II) hydride toward unsaturated molecules. Reactions of $\mathrm{LGeH}(\mathbf{5})$ and $\mathrm{LSnH}(\mathbf{2 0})$ with gaseous carbon dioxide resulted in the formation of the germanium(II) and tin(II) ester of formic acid (6) and (21) respectively, which represent a new class of compounds of group 14 elements. For example a low valent stable carbon analogue of composition $\mathrm{RC}-\mathrm{O}-\mathrm{C}(\mathrm{O}) \mathrm{H}$ has so far not been reported. A RC-O-C(O)H species should be extremely unstable and rearrange to the corresponding 1,2-dicarbonyl compound, $\mathrm{RC}(\mathrm{O})-\mathrm{C}(\mathrm{O}) \mathrm{H}$. Compounds $\mathbf{6}$ and $\mathbf{2 1}$ are potential $\mathrm{C} 1$ synthons for further synthesis of organic compounds.

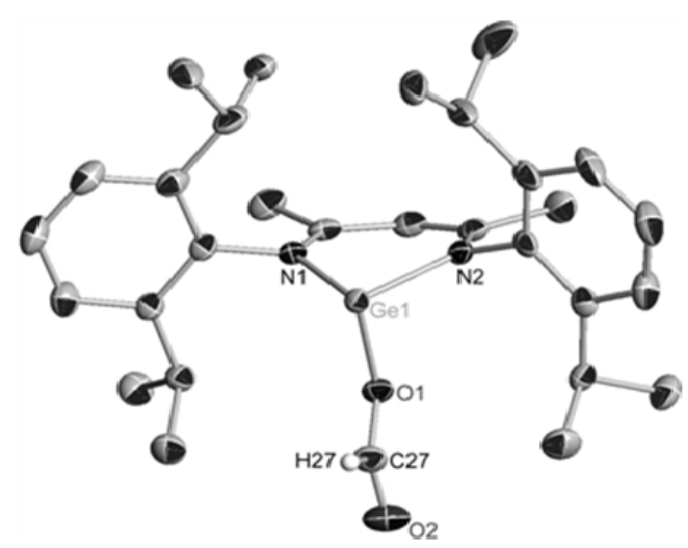

6

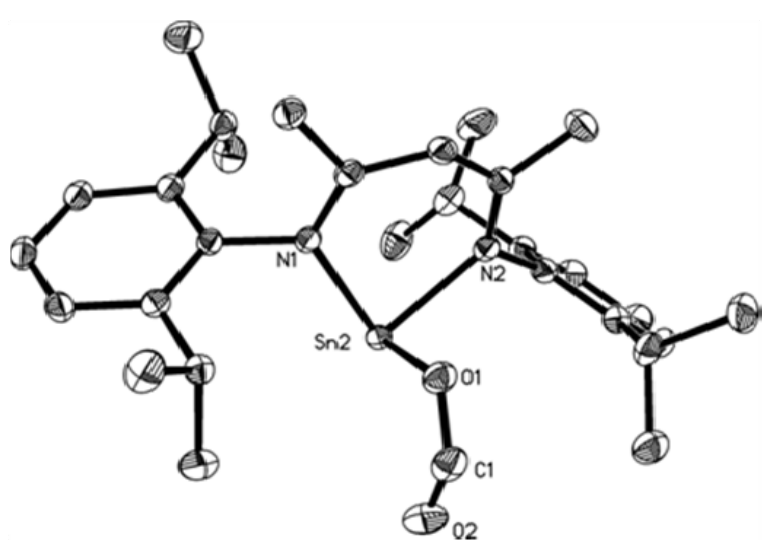

21

Germanium(II) and tin(II) alkoxides have been prepared by the reaction of $\mathbf{5}$ and $\mathbf{2 0}$ with carbonyl compounds with the outcome of compounds containing the $\mathrm{M}(\mathrm{II})-\mathrm{O}-\mathrm{CH}(\mathrm{M}=\mathrm{Ge}$ and Sn) core. $\mathrm{LGeH}(\mathbf{5})$ reacts only with activated carbonyl compounds, namely 2,2,2trifluoro acetophenone and 2,2,2-trifluoroacetothiophane (7-8), which is in contrast to LSnH (20). Compound 20 reacts with a variety of carbonyl compounds such as ferrocene carbaldehyde, benzophenone etc. (22-26, 35-40). 


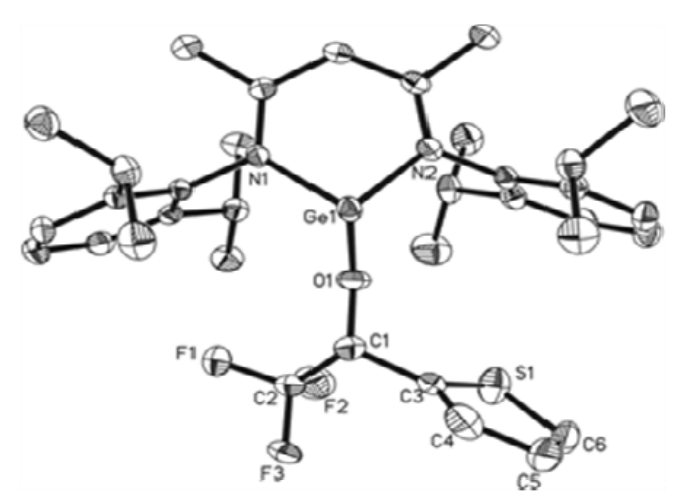

8

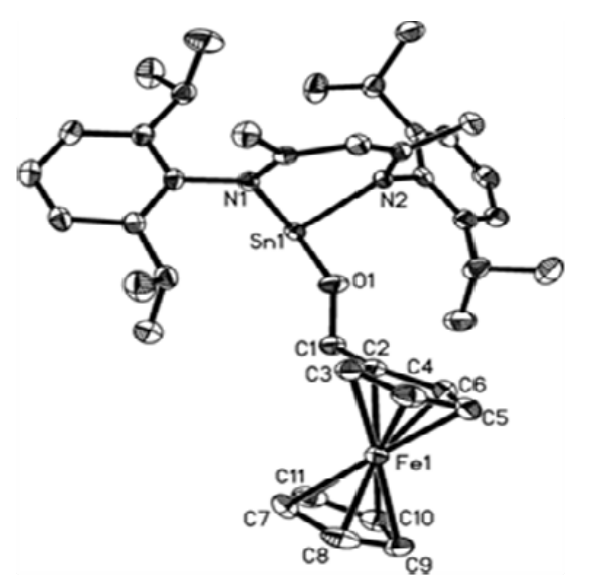

22

The reaction of $\mathbf{5}$ and $\mathbf{2 0}$ with terminal and internal alkines generated the vinyl substituted germylene and stannylene $(\mathbf{9 - 1 3}, \mathbf{2 7 - 3 2})$ rather than products under elimination of dihydrogen in the case of terminal alkines. These vinyl substituted alkenes have a lone pair of electrons on the germanium or tin center and a carbon carbon double bond. They are prone for further reactions. The unique pathways of these reactions are the simultaneous transfer of hydrogen and germylene or stannylene from $\mathbf{5}$ and $\mathbf{2 0}$ respectively.

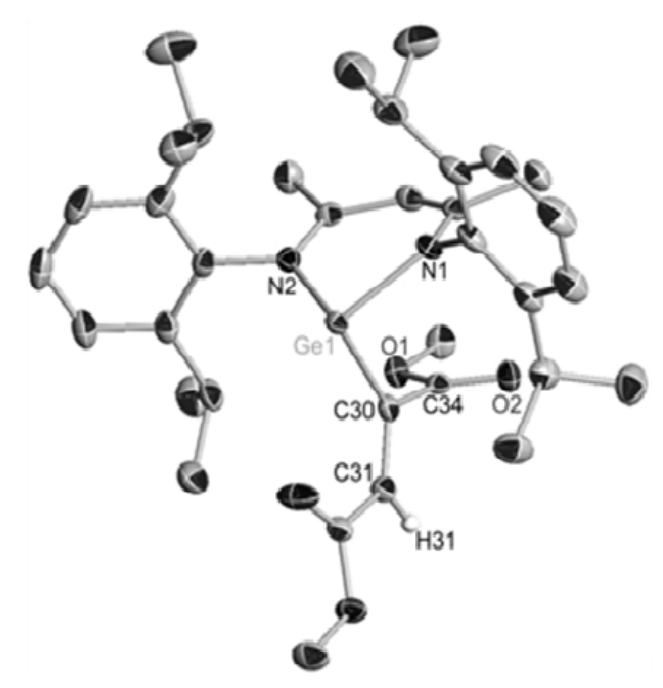

11

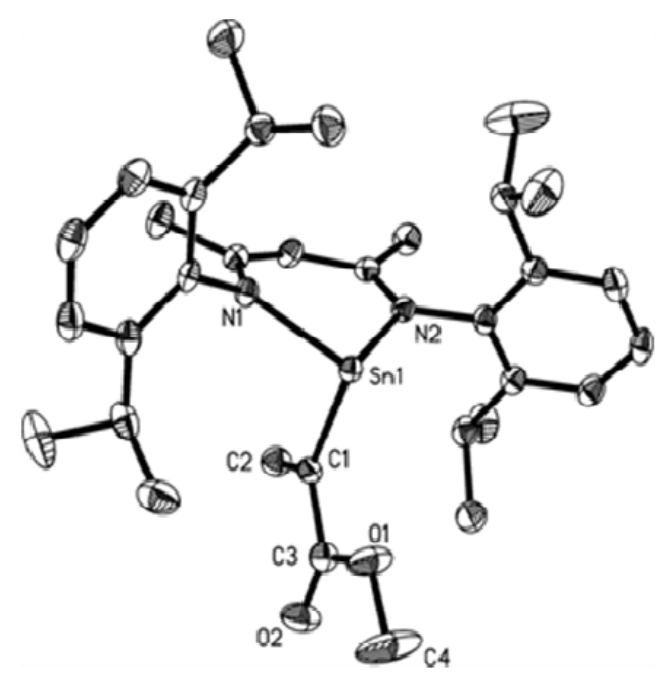

27

5 also reacts with diethyl azodicarboxylate (DEAD) under oxidative addition to give the germylene substituted hydrazine compound 14. Germanium(II) hydroxide, LGeOH (15), has been prepared by the reaction of $\mathrm{LGeH}$ with nitrous oxide as a mono oxygen source. The reaction of 5 with elemental sulfur resulted in the formation of a germanium analogue of dithiocarboxylic acid (16), which represents a new precursor for many metal complexes. 


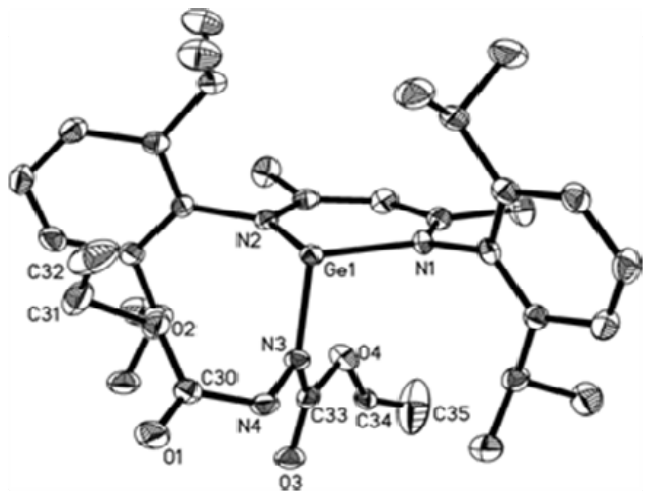

14

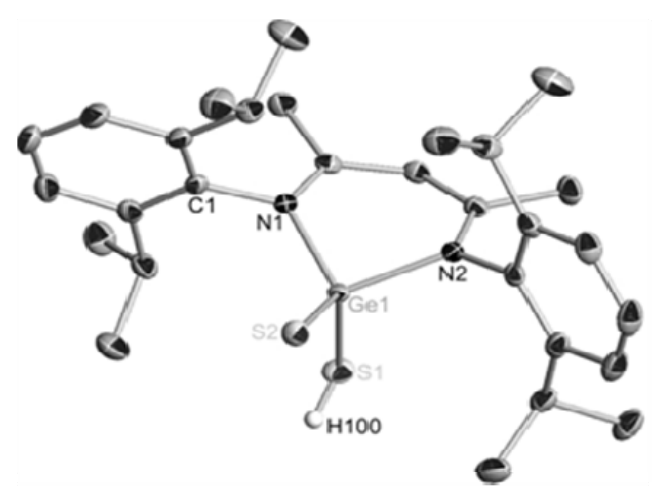

16

The corresponding reaction of $\mathrm{LGeH}$ with trimethylsilyl azide resulted in the formation of two compounds, the terminal germanium(II) azide (17) and germanium(IV) diamide (18), under migration of a hydrogen atom from a methyl group of the ligand backbone to the coordinate $\mathrm{N}$ atom in the latter case. The formation of compounds $\mathbf{1 6}$ and $\mathbf{1 8}$ involves the change of oxidation state of germanium from two to four.

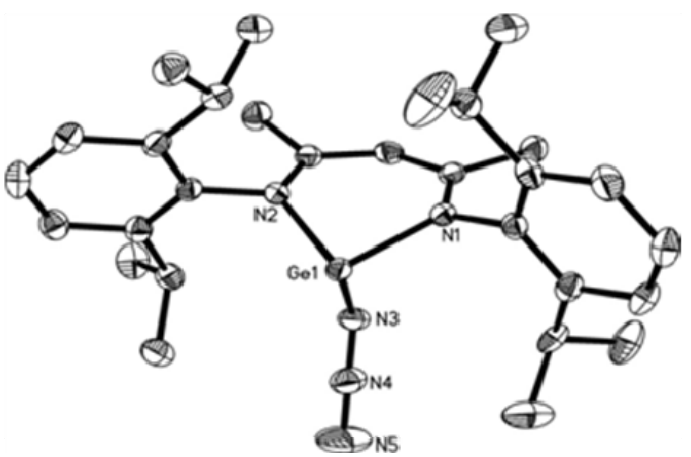

17

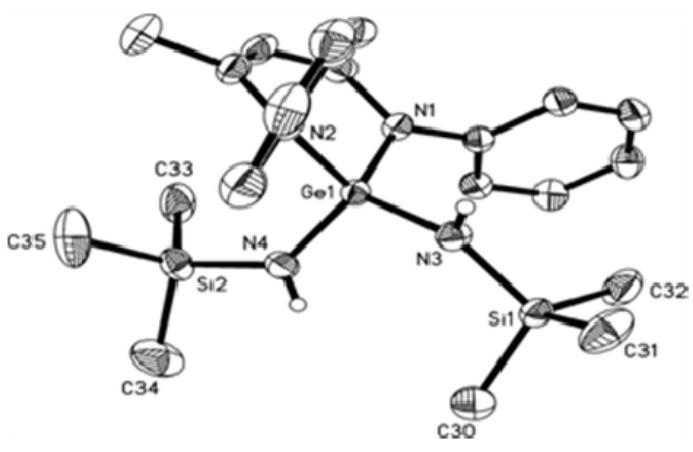

18

There are striking differences between the reactions of LSnH (20) with and without fluorinated carbonyl compounds. There is a competition between the addition reaction $v s$ exchange reaction. Considering 4-fluoro benzophenone and pentafluoro benzaldehyde as precursors the carbonyl group is more prone to addition reaction (39-40) than to the exchange reaction of fluorine of a C-F bond with $\mathrm{LSnH}$. In the case of pentafluoro benzophenone and perfluoro benzophenone there is a competition between the addition $v s$ exchange reaction. Two products are formed with the selective para fluorine exchange under formation of LSnF (1). In this context it is interesting to mention that the bond energy of the aliphatic C-F bond is smaller than that of the aromatic C-F bond, although there are exclusively addition reactions to the trifluoro acetyl group (35-36) rather than the exchange reaction of $\mathrm{C}-\mathrm{F}$ bond with LSnH (20). 


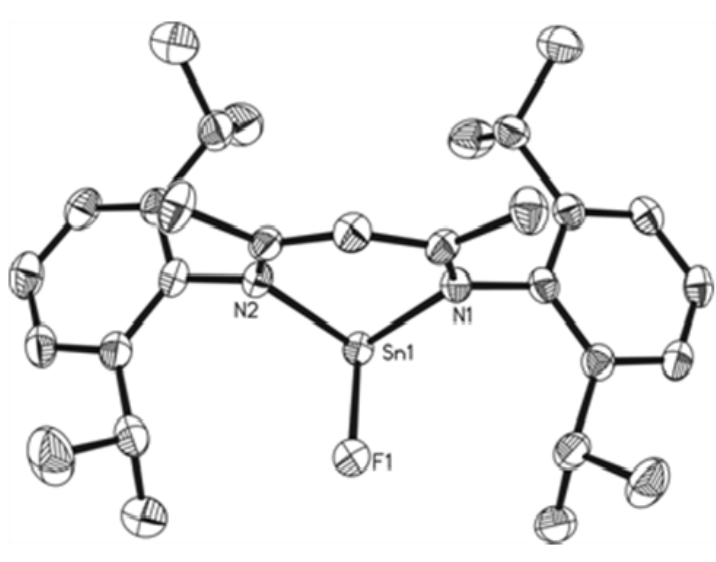

1

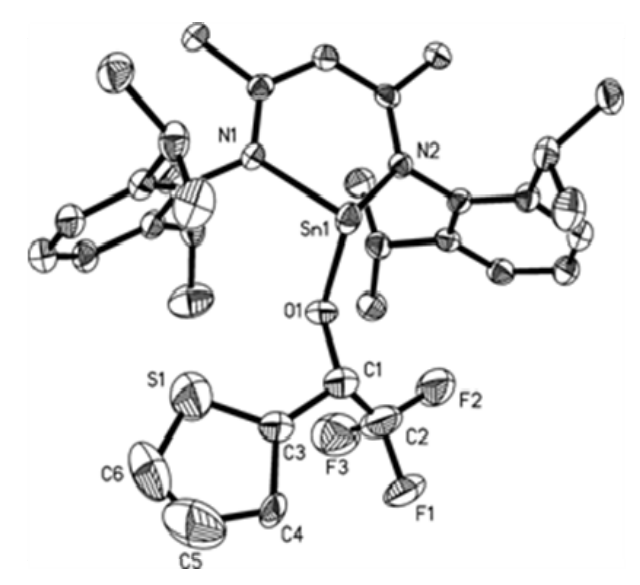

36

Moreover it was shown that the synthesis of the N-heterocyclic germylene L'Ge (19) achieved the dehydrogenation of $\mathrm{LGeH}(\mathbf{5})$ by using a frustrated carbene borane Lewis pair. The sequential cleavage of $\mathrm{C}-\mathrm{H}$ and $\mathrm{Ge}-\mathrm{H}$ bonds without a catalyst is shown herein. Compound 19 is also formed by the reaction of 1,3-di-tert-butylimidazol-2-ylidene with $\mathrm{LGeCl}$ or LGeOTf (63) under the activation of the $\mathrm{C}-\mathrm{H}$ bond of one of the methyl groups of the ligand backbone.

Herein we have also shown that the germylene, L'Ge (19) and silylene, L'Si (50) have striking differences in their reactivity. 19 reacts with $\mathrm{N}-\mathrm{H}$ and $\mathrm{O}-\mathrm{H}$ bonded compounds including gaseous ammonia and water under formation of stable unique germanium(II) compounds $(\mathbf{1 5}, \mathbf{4 2 - 4 6})$, demonstrating an example of sustainable chemistry. L'Si (50) reacts with ammonia to afford compound $\mathbf{5 1}$ under oxidative addition at the silicon(II) atom. The result reported here shows that the silylene can mimic to some extent the behavior of a transition metal. The oxidative addition of $\mathrm{NH}_{3}$ under very mild conditions offers an alternative paradigm in the ongoing search for catalytic systems capable of transforming ammonia efficiently into useful amino compounds. Moreover diphenyl hydrazone reacts with the silylene $\mathbf{5 0}$ to form the non-aromatic siloxi-indolin-1-amine derivative $\mathbf{5 4}$ instead of an N$\mathrm{H}$ bond cleavage of the $\mathrm{NH}_{2}$ group. Here it is noticed that in the case of $\mathbf{1 9}$ there is no change of the oxidation state of germanium, whereas in the case of $\mathbf{5 0}$ there is always oxidative addition at the silicon center. 


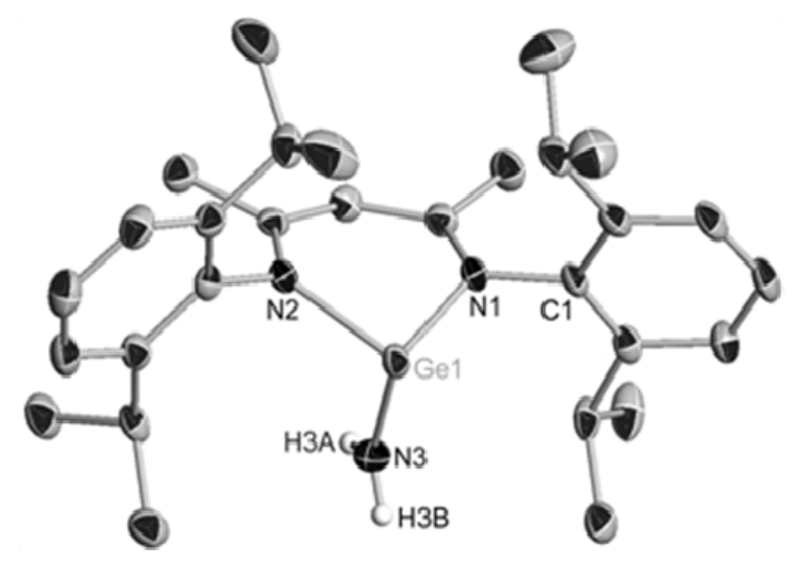

42

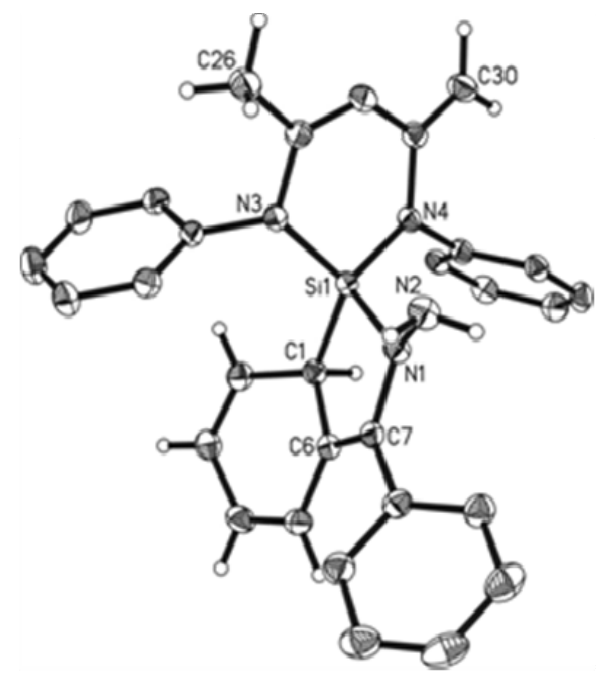

54

Moreover $\mathrm{H}_{3} \mathrm{NBH}_{3}$ delivered the dihydrogen to N-heterocyclic carbene, 1,3-di-tertbutylimidazol-2-ylidene as well as to germylene 19 at room temperature without any additional catalyst by oxidative addition. The latter examples demonstrate that the solid $\mathrm{H}_{3} \mathrm{NBH}_{3}$ can be used instead of gaseous hydrogen for hydrogenation reactions without any additional catalyst. In comparison to the carbon(II) in N-heterocyclic carbene, we found that the oxidation of the acyclic carbon carbon double bond in L'Ge (19) occured.

Germylene 19 also reacts with $\mathrm{B}\left(\mathrm{C}_{6} \mathrm{~F}_{5}\right)_{3}$ and trimethylsilyl diazomethane at room temperature to form the zwitterionic germanium(II) compound $\mathbf{4 8}$ under $\mathrm{C}-\mathrm{B}$ bond formation and diazogermylene compound $\left.\mathrm{LGeC}_{(} \mathrm{N}_{2}\right) \mathrm{SiMe}_{3}$ (58) by cleavage of a $\mathrm{C}-\mathrm{H}$ bond. The diazogermylene compound $\mathrm{LGeC}\left(\mathrm{N}_{2}\right) \mathrm{SiMe}_{3}$ (58) rearranges after about 3 months to the isonitriletrimethylsilyl germanium(II) amide $\mathrm{LGeN}\left(\mathrm{SiMe}_{3}\right) \mathrm{NC}(\mathbf{5 9})$.

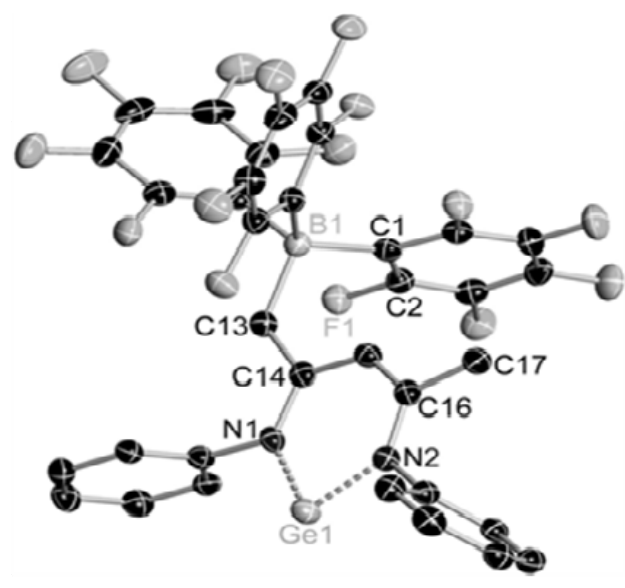

48

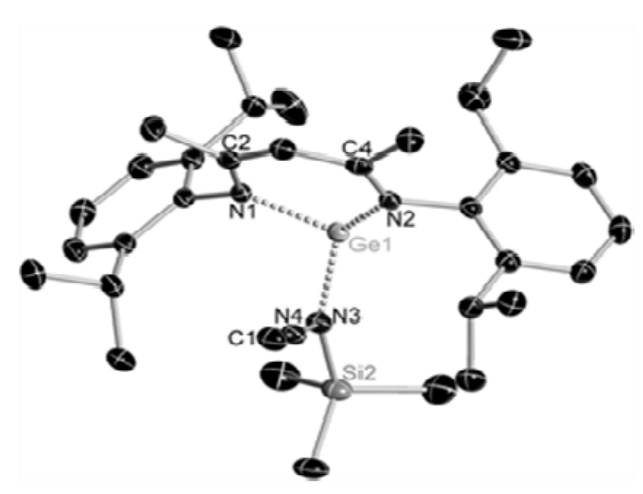

59 
The reaction of ethyl diazoacetate and trimethylsilyl diazomethane with $\mathbf{5}$ leads to the first stable germanium(II) substituted hydrazone derivatives, LGeN(H)NCHR, $\left(\mathrm{R}=\mathrm{CO}_{2} \mathrm{Et}\right.$ (55), $\left.\mathrm{SiMe}_{3}(\mathbf{5 6})\right)$ in high yield. In this reaction we are showing for the first time the unprecedented end-on nitrogen insertion of a diazo compound into a germanium(II) hydrogen bond. The reaction of a methyl analogue, LGeMe, with trimethylsilyl diazomethane forms the germanium(IV) amide, $\mathrm{L}^{\prime} \mathrm{Ge}(\mathrm{Me}) \mathrm{NHNCHSiMe}_{3}$ (57) with migration of hydrogen from the methyl group of the ligand backbone. Finally the reaction of the lithium salt of trimethylsilyl diazomethane with $\mathrm{LSnCl}$ yields the $\mathrm{LSn}-\mathrm{N}=\mathrm{C}=\mathrm{N}-\mathrm{SiMe}_{3}(\mathbf{6 0})$ under migration of the silyl group. This result shows that for the first time a compound with the unique $\mathrm{Sn}$ (II) substituted carbodiimide was isolated.

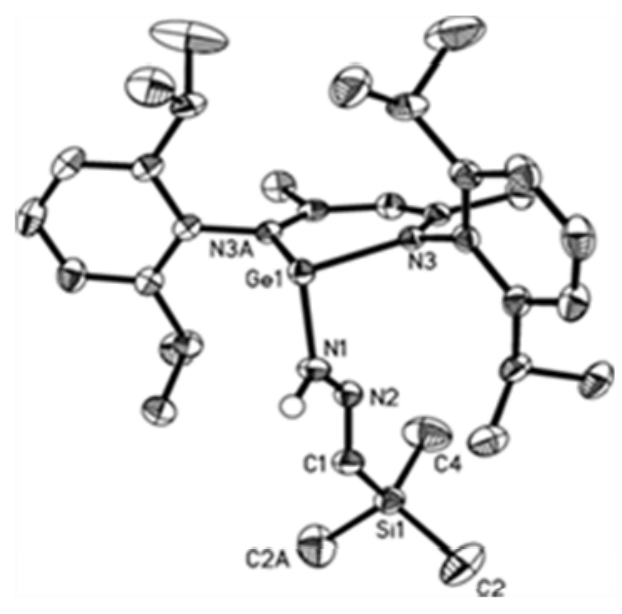

56

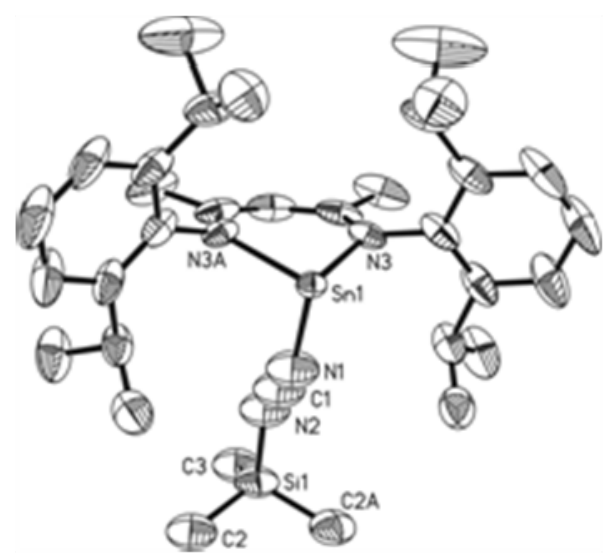

60

Similar to the transfer of the hydride described, the reaction of $\mathbf{5}$ and $\mathbf{2 0}$ with unsaturated compounds, leads to the transfer of " $\mathrm{NMe}_{2}$ " when $\mathrm{LSnNMe}_{2}(\mathbf{6 6})$ is used. Compound 66 reacts with ketones to generate tin(II)-alkoxide compounds (67-68) containing the $\mathrm{Sn}(\mathrm{II})-\mathrm{O}-$ $\mathrm{CNMe}_{2}$ core. Furthermore tin(II)-dimethylamide reacts with terminal alkines generating the alkinyl substituted stannylene (69-70) under elimination of dimethyl amine rather than the insertion of $\mathrm{Sn}-\mathrm{NMe}_{2}$ into the carbon carbon triple bond, which is observed in the reaction of $\mathbf{5}$ and $\mathbf{2 0}$ with terminal alkines. 


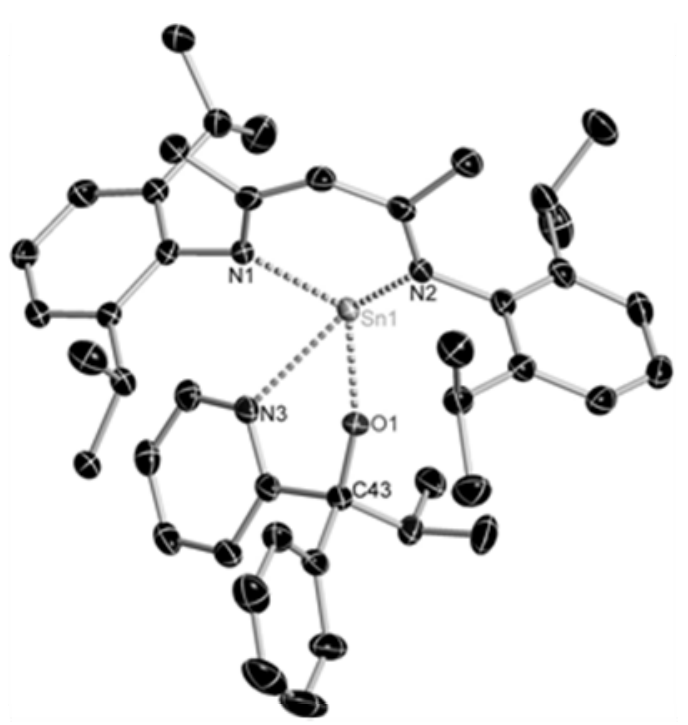

67

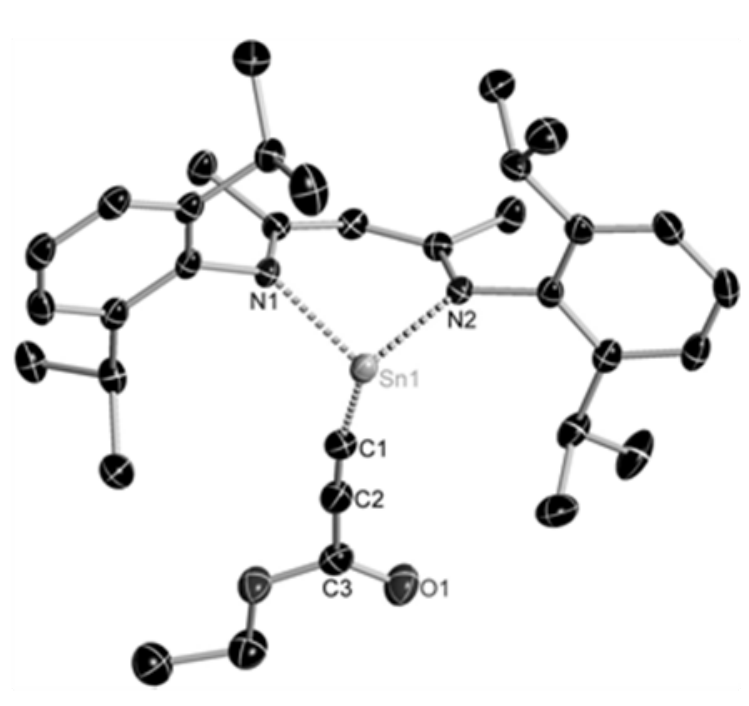

70

Finally we have prepared the monomeric tin(II) hydroxide, $\mathrm{LSn}(\mathrm{OH}) \mathrm{Fe}(\mathrm{CO})_{4}$ (73) for the first time under the protection of a metal fragment that shielded the tin(II) center to avoid protonation at the tin. The synthesis of $\mathbf{7 3}$ might have a similar impact on molecular tin(II) chemistry as this has been shown for its analogue $\mathrm{LGeOH}$.

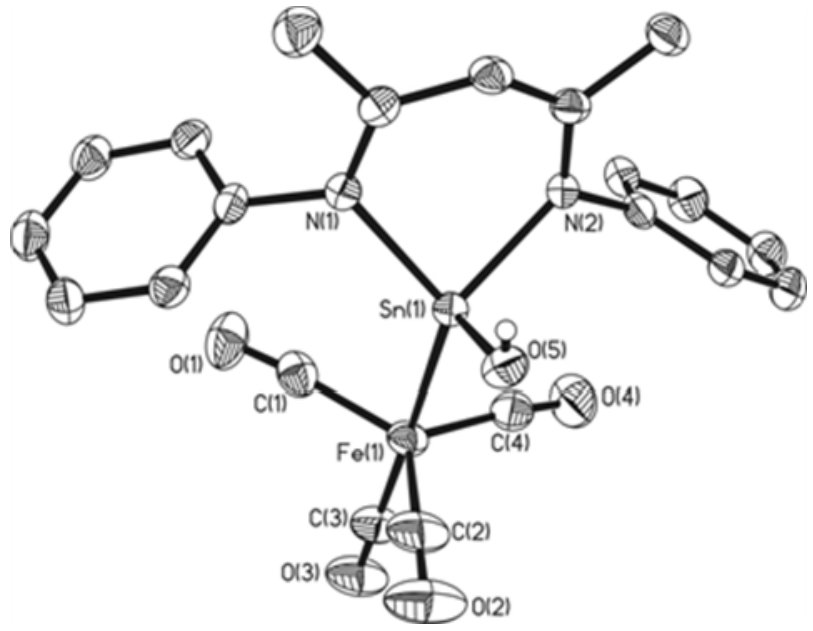

73

It is interesting to mention that most of the compounds are stable at room temperature and highly soluble in common organic solvents and therefore favor their further functionalization as metal complexes and metal catalysts. 


\section{Chapter 11 \\ Experimental Section \\ 11.1. General Procedures}

All reactions and handling of reagents were performed under an atmosphere of dry nitrogen or argon using Schlenk techniques or a glovebox where the $\mathrm{O}_{2}$ and $\mathrm{H}_{2} \mathrm{O}$ levels were usually kept bellow 1 ppm. All glassware was oven-dried at $160{ }^{\circ} \mathrm{C}$ for at least $24 \mathrm{~h}$, assembled hot and cooled under high vacuum prior to use. Toluene, benzene, $n$-hexane, $n$-pentane, tetrahydrofuran, and diethylether were purified with the M-Braun solvent drying system. Dichloromethane $\left(\mathrm{CaH}_{2}\right)$ were dried and distilled prior to use.

\subsection{Physical Measurements}

Melting points were measured in sealed glass tubes on a Büchi B-540 melting point apparatus.

NMR spectra were recorded on Bruker Avance 200, Bruker Avance 300, and Bruker Avance 500 NMR spectrometers. Downfield shifts from the reference are quoted positive; upfield shifts are assigned negative values for the case of external references. Deuterated NMR solvents $\mathrm{C}_{6} \mathrm{D}_{6}, \mathrm{C}_{7} \mathrm{H}_{8}$, and THF- $\mathrm{D}_{8}$ were dried by stirring for 2 days over $\mathrm{Na} / \mathrm{K}$ alloy followed by distillation in vacuo and degassed. $\mathrm{CD}_{2} \mathrm{Cl}_{2}$ stirring with $\mathrm{CaH}_{2}$ for $6 \mathrm{hrs}$ followed by filtration. Heteroatom NMR spectra were recorded ${ }^{1} \mathrm{H}$ decoupled with the exception of ${ }^{19} \mathrm{~F}$. Chemical shifts are reported in ppm with reference to residual deutorited solvent peak (internal) for ${ }^{1} \mathrm{H}$ nuclei, $\mathrm{SnMe}_{4}$ (external) for ${ }^{119} \mathrm{Sn}$ nuclei, $\mathrm{CFCl}_{3}$ (external) for ${ }^{19} \mathrm{~F}$ nuclei, $\mathrm{BF}_{3} \cdot \mathrm{OEt}_{2}$ (external) for ${ }^{11} \mathrm{~B}$ nuclei, $85 \%$ $\mathrm{H}_{3} \mathrm{PO}_{4}$ (external) for ${ }^{31} \mathrm{P}$ nuclei, $\mathrm{SiMe}_{4}$ (external) for ${ }^{29} \mathrm{Si}$ nuclei.

IR spectra were recorded on a Bio-Rad Digilab FTS7 spectrometer in the range $4000-350 \mathrm{~cm}^{-1}$ as $\mathrm{KBr}$ pellets. Only the absorption of significant moieties $(\mathrm{N}-\mathrm{H}, \mathrm{O}-\mathrm{H}, \mathrm{O}-\mathrm{D}, \mathrm{CO}, \mathrm{C}=\mathrm{C})$ are assigned.

Mass spectra were obtained with a Finnigan MAT 8230 or a Varian MAT CH5 instrument (70 eV) by EI-MS methods.

Elemental analyses were performed by the Analytisches Labor des Instituts für Anorganische Chemie der Universität Göttingen.

\subsection{Starting Materials}

$\operatorname{MeLi}\left(1.6 \mathrm{M}\right.$ in $\mathrm{Et}_{2} \mathrm{O}$, Aldrich), $\mathrm{LiNMe}_{2}$ (Aldrich), $\mathrm{KN}\left(\mathrm{SiMe}_{3}\right)_{2}$ (Aldrich), $\mathrm{GeCl}_{2} \cdot$ di-oxane (Aldrich), $\mathrm{SnCl}{ }_{2}$ (Aldrich), $\mathrm{B}\left(\mathrm{C}_{6} \mathrm{~F}_{5}\right)_{3}$ (TCI Organic Chemicals), $\mathrm{N}_{2} \mathrm{CHCO}_{2} \mathrm{Et}$ (Aldrich), $\mathrm{N}_{2} \mathrm{CHSiMe}_{3}$ (Aldrich), $\mathrm{N}_{3} \mathrm{SiMe}_{3}$ (Aldrich), $\mathrm{Fe}_{2}(\mathrm{CO})_{9}$ (Aldrich), sulfur (Aldrich), AgOTf (Aldrich), $\mathrm{HCCCO}_{2} \mathrm{Me}$ (Aldrich), $\mathrm{HCCCO}_{2} \mathrm{Et}$ (Aldrich), $\mathrm{MeCCCO}_{2} \mathrm{Et}$ (Aldrich), $\mathrm{MeO}_{2} \mathrm{CCCCO}_{2} \mathrm{Me}$ (Aldrich), $\mathrm{EtO}_{2} \mathrm{CCCCO}_{2} \mathrm{Et}$ (Aldrich), $t \mathrm{BuO}_{2} \mathrm{CCCCO}_{2} t \mathrm{Bu}$ (Aldrich), $\mathrm{EtO}_{2} \mathrm{CNNCO}_{2} \mathrm{Et}$ (Aldrich), $i \mathrm{PrNCN} i \mathrm{Pr}$ (Aldrich), CyNCNCy (Aldrich), FcCHO (Aldrich), $\mathrm{Ph}_{2} \mathrm{CO}$ (Aldrich), $\mathrm{PhCO}$ (2-py) (Aldrich), (2-Py) $)_{2} \mathrm{CO}$ (Aldrich), $\left(\mathrm{C}_{3} \mathrm{H}_{5}\right)_{2} \mathrm{CO}$ (Aldrich), $\mathrm{PhCOCF}_{3}$ (Aldrich), (2- $\left.\mathrm{C}_{4} \mathrm{H}_{3} \mathrm{~S}\right) \mathrm{COCF}_{3}$ (Aldrich), $\mathrm{PhCOC}_{6} \mathrm{~F}_{5}$ (Aldrich), $\left(\mathrm{C}_{6} \mathrm{~F}_{5}\right)_{2} \mathrm{CO}$ (Aldrich), $\mathrm{PhCO}\left(4-\mathrm{C}_{6} \mathrm{H}_{4} \mathrm{~F}\right)$ (Aldrich), and $\mathrm{C}_{6} \mathrm{~F}_{5} \mathrm{CHO}$ (Aldrich) were

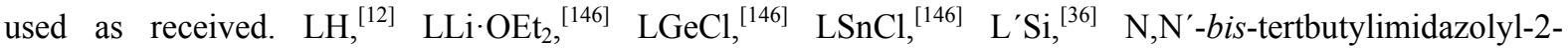
ylidene, ${ }^{[191]}$ and $\mathrm{AlH}_{3} \cdot \mathrm{NMe}_{3},{ }^{[155]}$ were prepared by literature procedures. Demineralized, degassed $\mathrm{H}_{2} \mathrm{O}$ was used for hydrolysis experiments.

\subsection{Syntheses of Compounds}

\subsubsection{Synthesis of $\operatorname{LSnF}$ (1)}

Method A: A solution of LSnMe $(0.550 \mathrm{~g}, 1 \mathrm{mmol})$ in toluene $(20 \mathrm{~mL})$ was added to a stirred suspension of $\mathrm{Me}_{3} \mathrm{SnF}(0.200 \mathrm{~g}, 1.1 \mathrm{mmol})$ in toluene $(10 \mathrm{~mL})$, and the reaction mixture was refluxed for $3 \mathrm{~h}$. After removal of all the volatiles the residue was extracted with $n$-hexane $(30 \mathrm{~mL})$ and concentrated to about $10 \mathrm{~mL}$ and stored in a $-30{ }^{\circ} \mathrm{C}$ freezer. Colorless crystals of 1 suitable for X-ray diffraction analysis are formed after one day. Yield: $0.470 \mathrm{~g}(85 \%)$. Method B: A solution of $\operatorname{LSnN}\left(\mathrm{SiMe}_{3}\right)_{2}(0.700 \mathrm{~g}, 1 \mathrm{mmol})$ in toluene $(20 \mathrm{~mL})$ was added to a stirred suspension of $\mathrm{Me}_{3} \mathrm{SnF}(0.200 \mathrm{~g}, 1.1 \mathrm{mmol})$ in toluene $(10 \mathrm{~mL})$, and the reaction mixture was refluxed for $3 \mathrm{~h}$. After removal of all the volatiles the residue was extracted with $n$-hexane $(30 \mathrm{~mL})$ and the resulting solution was concentrated to about $10 \mathrm{~mL}$ and stored in a $-30{ }^{\circ} \mathrm{C}$ freezer. Colorless crystals of 1 suitable for X-ray diffraction analysis are formed after one day. Yield: $0.390 \mathrm{~g}(70 \%)$. Mp $130{ }^{\circ} \mathrm{C} .{ }^{1} \mathrm{H}$ NMR $\left(500 \mathrm{MHz}, \mathrm{C}_{6} \mathrm{D}_{6}\right): \delta$ 7.04-7.17 (m, 6H, Ar- H ), 4.99 (s, 1H, $\gamma-\mathrm{CH}), 3.84$ (sept, 2H, $\left.\mathrm{CH}\left(\mathrm{CH}_{3}\right)_{2}\right), 3.09$ (sept, 2H, $\left.\mathrm{CH}\left(\mathrm{CH}_{3}\right)_{2}\right), 1.63(\mathrm{~s}$, $\left.6 \mathrm{H}, \mathrm{CH}_{3}\right), 1.41\left(\mathrm{~d}, 6 \mathrm{H}, \mathrm{CH}\left(\mathrm{CH}_{3}\right)_{2}\right), 1.18\left(\mathrm{q}, 12 \mathrm{H}, \mathrm{CH}\left(\mathrm{CH}_{3}\right)_{2}\right), 1.09\left(\mathrm{~d}, 6 \mathrm{H}, \mathrm{CH}\left(\mathrm{CH}_{3}\right)_{2}\right) \mathrm{ppm} .{ }^{19} \mathrm{~F}\left\{{ }^{1} \mathrm{H}\right\} \mathrm{NMR}$ 
(188.31 MHz, $\left.\mathrm{C}_{6} \mathrm{D}_{6}\right): \delta-125.29 \mathrm{ppm} .{ }^{119} \mathrm{Sn}\left\{{ }^{1} \mathrm{H}\right\}$ NMR (186.50 MHz, $\left.\mathrm{C}_{6} \mathrm{D}_{6}\right): \delta-371.52 \mathrm{ppm}$. EI-MS: $m / z(\%)$ 556 (20) [M] $]^{+}$. Anal. calcd for $\mathrm{C}_{29} \mathrm{H}_{41} \mathrm{FN}_{2} \mathrm{Sn}$ (556.23): C 62.72, H 7.44, N 5.04; Found: C 62.38, H 8.67, N 5.00.

11.4.2. Synthesis of $\mathrm{LGeBr}(2)$

A solution of $\operatorname{LLi}\left(\mathrm{OEt}_{2}\right)(0.498 \mathrm{~g}, 1.0 \mathrm{mmol})$ in diethyl ether $(20 \mathrm{~mL})$ was added dropwise to a stirred suspension of $\mathrm{GeBr}_{2}(0.230 \mathrm{~g}, 1.0 \mathrm{mmol})$ in diethyl ether $(10 \mathrm{~mL})$ at $-78{ }^{\circ} \mathrm{C}$. The reaction mixture was warmed to room temperature and was stirred for another $12 \mathrm{~h}$. The precipitate was filtered, and the solvent was partially reduced (ca. $15 \mathrm{~mL}$ ). Keeping the solution in a $-32{ }^{\circ} \mathrm{C}$ freezer for overnight afforded colorless crystals of $\mathbf{2}$, which are suitable for X-ray diffraction analysis. Yield: $0.480 \mathrm{~g}(85 \%)$. Mp $214{ }^{\circ} \mathrm{C} .{ }^{1} \mathrm{H} N M R\left(500 \mathrm{MHz}, \mathrm{C}_{6} \mathrm{D}_{6}\right)$ : $\delta$ 7.03-7.18 (m, 6H, $\mathrm{Ar}-H$ ), 5.22 (s, $1 \mathrm{H}, \gamma-\mathrm{CH}), 4.01$ (sept, $\left.2 \mathrm{H}, \mathrm{CH}\left(\mathrm{CH}_{3}\right)_{2}\right), 3.13$ (sept, $\left.2 \mathrm{H}, \mathrm{CH}\left(\mathrm{CH}_{3}\right)_{2}\right), 1.59$ (s, $\left.6 \mathrm{H}, \mathrm{CH}_{3}\right), 1.48$ (d, $\left.6 \mathrm{H}, \mathrm{CH}\left(\mathrm{CH}_{3}\right)_{2}\right), 1.20$ (q, $\left.12 \mathrm{H}, \mathrm{CH}\left(\mathrm{CH}_{3}\right)_{2}\right), 1.00$ (d, 6H, CH(CH $\left.\mathrm{CH}_{3}\right)$ ppm. EI-MS $(70 \mathrm{eV}): \mathrm{m} / \mathrm{z}$ (\%): 570 (100) [M] ${ }^{+}$. Anal. calcd for $\mathrm{C}_{29} \mathrm{H}_{41} \mathrm{BrGeN}_{2}$ (570.17): C 61.09, H 7.25, N 4.91; Found: C 60.46, H 7.10, N 4.87 .

11.4.3. Synthesis of $\operatorname{LSnBr}(3)$

A solution of $\mathrm{LLi}\left(\mathrm{OEt}_{2}\right)(0.498 \mathrm{~g}, 1.0 \mathrm{mmol})$ in diethyl ether $(20 \mathrm{~mL})$ was added dropwise to a stirred suspension of $\mathrm{SnBr}_{2}(0.280 \mathrm{~g}, 1.0 \mathrm{mmol})$ in diethyl ether $(10 \mathrm{~mL})$ at $-78{ }^{\circ} \mathrm{C}$. The reaction mixture was warmed to room temperature and was stirred for another $12 \mathrm{~h}$. The precipitate was filtered, and the solvent was partially reduced (ca. $20 \mathrm{~mL}$ ). StoraSn of the remaining solution in a $-32{ }^{\circ} \mathrm{C}$ freezer for overnight afforded colorless crystals of 3 suitable for X-ray diffraction analyses. Yield: $0.490 \mathrm{~g}(80 \%)$. Mp $206{ }^{\circ} \mathrm{C} .{ }^{1} \mathrm{H}$ NMR ( $500 \mathrm{MHz}$, $\left.\mathrm{C}_{6} \mathrm{D}_{6}\right): \delta$ 7.02-7.17 (m, 6H, Ar- $H$ ), $5.11(\mathrm{~s}, 1 \mathrm{H}, \gamma-\mathrm{CH}), 3.99$ (sept, $\left.2 \mathrm{H}, \mathrm{CH}\left(\mathrm{CH}_{3}\right)_{2}\right), 3.11$ (sept, $\left.2 \mathrm{H}, \mathrm{CH}\left(\mathrm{CH}_{3}\right)_{2}\right)$, $1.61\left(\mathrm{~s}, 6 \mathrm{H}, \mathrm{CH}_{3}\right), 1.44$ (d, 6H, $\left.\mathrm{CH}\left(\mathrm{CH}_{3}\right)_{2}\right), 1.19$ (q, $\left.12 \mathrm{H}, \mathrm{CH}\left(\mathrm{CH}_{3}\right)_{2}\right), 1.03\left(\mathrm{~d}, 6 \mathrm{H}, \mathrm{CH}\left(\mathrm{CH}_{3}\right)_{2}\right) \mathrm{ppm} .{ }^{119} \mathrm{Sn}\left\{{ }^{1} \mathrm{H}\right\}$ NMR $\left(186.50 \mathrm{~Hz}, \mathrm{C}_{6} \mathrm{D}_{6}\right): \delta-180.47 \mathrm{ppm}$. EI-MS $(70 \mathrm{eV}): \mathrm{m} / \mathrm{z}(\%): 616(100)[\mathrm{M}]^{+}$. Anal. calcd for $\mathrm{C}_{29} \mathrm{H}_{41} \mathrm{BrN}_{2} \mathrm{Sn}$ (616.15): C 56.52, H 6.71, N 4.55. Found: C 55.58, H 6.55, N 4.42.

11.4.4. Synthesis of LSnI (4)

A solution of $\operatorname{LLi}\left(\mathrm{OEt}_{2}\right)(0.498 \mathrm{~g}, 1 \mathrm{mmol})$ in diethyl ether $(20 \mathrm{~mL})$ was added drop by drop to a stirred suspension of $\mathrm{SnI}_{2}(0.375 \mathrm{~g}, 1 \mathrm{mmol})$ in diethyl ether $(10 \mathrm{~mL})$ at $-78{ }^{\circ} \mathrm{C}$. The reaction mixture was warmed to room temperature and stirring was continued for another $12 \mathrm{~h}$. The precipitate was filtered and the solvent was partially reduced (ca. $20 \mathrm{~mL}$ ). Keeping the remaining solution in a $-32{ }^{\circ} \mathrm{C}$ freezer, afforded colorless crystals of 4 suitable for X-ray diffraction analyses. Yield: $0.510 \mathrm{~g}(77 \%)$. Mp $197{ }^{\circ} \mathrm{C} .{ }^{1} \mathrm{H}$ NMR ( $\left.300 \mathrm{MHz}, \mathrm{C}_{6} \mathrm{D}_{6}\right): \delta 7.01-$ 7.15 (m, 6H, Ar- $H$ ) , 5.20 (s, $1 \mathrm{H}, \gamma-\mathrm{CH}), 4.05$ (sept, $\left.2 \mathrm{H}, \mathrm{CH}\left(\mathrm{CH}_{3}\right)_{2}\right), 3.09$ (sept, $\left.2 \mathrm{H}, \mathrm{CH}\left(\mathrm{CH}_{3}\right)_{2}\right), 1.59$ (s, $6 \mathrm{H}$, $\left.\mathrm{CH}_{3}\right), 1.42\left(\mathrm{~d}, 6 \mathrm{H}, \mathrm{CH}\left(\mathrm{CH}_{3}\right)_{2}\right), 1.17\left(\mathrm{q}, 12 \mathrm{H}, \mathrm{CH}\left(\mathrm{CH}_{3}\right)_{2}\right), 1.06\left(\mathrm{~d}, 6 \mathrm{H}, \mathrm{CH}\left(\mathrm{CH}_{3}\right)_{2}\right)$ ppm. ${ }^{119} \mathrm{Sn}\left\{{ }^{1} \mathrm{H}\right\} \mathrm{NMR}$ $\left(112.00 \mathrm{~Hz}, \mathrm{C}_{6} \mathrm{D}_{6}\right): \delta-107.33$ ppm. EI-MS $(70 \mathrm{eV}): \mathrm{m} / z(\%): 663(100)[\mathrm{M}]^{+}$. Anal. calcd for $\mathrm{C}_{29} \mathrm{H}_{41} \mathrm{IN}_{2} \mathrm{Sn}(664)$ : C 52.51, H 6.23, N 4.22; Found: C 51.55, H 5.78, N 4.06.

11.4.5. Synthesis of LGeH (5)

Method A: A solution of $\mathrm{K}\left[\mathrm{HB}(\sec \mathrm{Bu})_{3}\right]$ in THF $(2.00 \mathrm{~mL}, 1 \mathrm{M}$, in THF) was slowly added drop by drop to a stirred solution of $\mathrm{LGeCl}(1.050 \mathrm{~g}, 2 \mathrm{mmol})$ in toluene $(30 \mathrm{~mL})$ at $-10^{\circ} \mathrm{C}$. The reaction mixture was warmed to room temperature and was stirred for additional $3 \mathrm{~h}$. After removal of all the volatiles, the residue was extracted with $n$-hexane $(30 \mathrm{~mL})$ and concentrated to about $10 \mathrm{~mL}$ and stored in a $-30{ }^{\circ} \mathrm{C}$ freezer. Red crystals of 5 are formed after one day. Yield: $0.80 \mathrm{~g}(88 \%)$. Method B: L'Ge (19) $(0.490 \mathrm{~g}, 1 \mathrm{mmol})$ and $\mathrm{NH}_{3} \mathrm{BH}_{3}(0.030 \mathrm{~g}, 1.0$ $\mathrm{mmol})$ were dissolved in toluene $(20 \mathrm{~mL})$ at room temperature. The color of the reaction mixture changed from brown red to red. The reaction mixture was stirred further for one hour. Then the solvent was removed in vacuo and the residue was extracted with $n$-hexane $(30 \mathrm{~mL})$. Yield: $0.42 \mathrm{~g}(85 \%)$. Mp $170{ }^{\circ} \mathrm{C} .{ }^{1} \mathrm{H}$ NMR $(200 \mathrm{MHz}$, $\left.\mathrm{C}_{6} \mathrm{D}_{6}\right): \delta 7.10-7.15(\mathrm{~m}, 6 \mathrm{H}, \mathrm{Ar}-H), 4.86(\mathrm{~s}, 1 \mathrm{H}, \gamma-\mathrm{CH}), 3.59$ (sept, $\left.2 \mathrm{H}, \mathrm{CH}\left(\mathrm{CH}_{3}\right)_{2}\right), 3.37$ (sept, $\left.2 \mathrm{H}, \mathrm{CH}\left(\mathrm{CH}_{3}\right)_{2}\right)$, $1.60\left(\mathrm{~s}, 6 \mathrm{H}, \mathrm{CH}_{3}\right), 1.35$ (d, 6H, $\left.\mathrm{CH}\left(\mathrm{CH}_{3}\right)_{2}\right), 1.22\left(\mathrm{~d}, 6 \mathrm{H}, \mathrm{CH}\left(\mathrm{CH}_{3}\right)_{2}\right), 1.19\left(\mathrm{~d}, 12 \mathrm{H}, \mathrm{CH}\left(\mathrm{CH}_{3}\right)_{2}\right), \mathrm{ppm}$.

11.4.6. Synthesis of $\mathrm{LGeOC}(\mathrm{O}) \mathrm{H}(6)$

In a $100 \mathrm{~mL}$ Schlenk flask a solution of $5(0.490 \mathrm{~g}, 1.00 \mathrm{mmol})$ in $20 \mathrm{~mL}$ toluene was treated with dry carbon dioxide gas at room temperature. After the addition of carbon dioxide under stirring the reaction mixture slowly 
changed the color from red to yellow and stirring was continued for $15 \mathrm{~min}$. After removal of all the volatiles, the residue was extracted with $n$-hexane $(20 \mathrm{~mL})$ and concentrated to about $10 \mathrm{~mL}$ and stored in a $-30{ }^{\circ} \mathrm{C}$ freezer. Yellow crystals of 6 suitable for X-ray diffraction analysis are formed after one day. Yield $(0.48 \mathrm{~g}, 90$ \%). Mp $183{ }^{\circ} \mathrm{C} .{ }^{1} \mathrm{H}$ NMR $\left(500 \mathrm{MHz}, \mathrm{C}_{6} \mathrm{D}_{6}\right): \delta 8.64(\mathrm{~s}, 1 \mathrm{H}, \mathrm{CH}), 7.05-7.19(\mathrm{~m}, 6 \mathrm{H}, \mathrm{Ar}-H), 5.06(\mathrm{~s}, 1 \mathrm{H}, \gamma-\mathrm{CH})$, 3.53 (sept, 2H, $\left.\mathrm{CH}\left(\mathrm{CH}_{3}\right)_{2}\right), 3.08$ (sept, 2H, $\left.\mathrm{CH}\left(\mathrm{CH}_{3}\right)_{2}\right), 1.54$ (s, 6H, $\mathrm{CH}$ ), 1.28 (d, 6H, $\left.\mathrm{CH}\left(\mathrm{CH}_{3}\right)_{2}\right), 1.20$ (d, $6 \mathrm{H}$, $\left.\mathrm{CH}\left(\mathrm{CH}_{3}\right)_{2}\right), 1.14\left(\mathrm{~d}, 6 \mathrm{H}, \mathrm{CH}\left(\mathrm{CH}_{3}\right)_{2}\right), 1.12\left(\mathrm{~d}, 6 \mathrm{H}, \mathrm{CH}\left(\mathrm{CH}_{3}\right)_{2}\right)$ ppm. IR (Nujol, $\left.\mathrm{KBr}\right): \widetilde{v}=2854(\mathrm{C}-\mathrm{H}), 1690 \mathrm{~cm}$ ${ }^{-1}(\mathrm{C}=\mathrm{O})$. EI-MS $(70 \mathrm{eV}): m / z(\%): 536(20)[\mathrm{M}]^{+}, 491(100)\left[\mathrm{M}-\mathrm{HCO}_{2}\right]^{+}$. Anal. calcd for $\mathrm{C}_{30} \mathrm{H}_{42} \mathrm{GeN}_{2} \mathrm{O}_{2}$ (535.25): C 67.31, H 7.91, N 5.23; Found: C 66.87, H 8.85, N 5.21.

\subsubsection{Synthesis of $\mathrm{LGeOCHPh}\left(\mathrm{CF}_{3}\right)$ (7)}

A solution of 2,2,2-trifluoro acetophenone $(0.175 \mathrm{~g}, 1.00 \mathrm{mmol}$ in $5 \mathrm{~mL}$ toluene) was added by cannula to a solution of $5(0.49 \mathrm{~g}, 1.00 \mathrm{mmol}$ in toluene $20 \mathrm{~mL})$ at room temperature. After $12 \mathrm{~h}$ all volatiles were removed in vacuo, and the remaining residue was extracted with $n$-hexane $(25 \mathrm{~mL})$. The solution was concentrated and kept in a freezer to obtain 7 as yellow crystals, which are suitable for X-ray diffraction analysis. Yield: $0.545 \mathrm{~g}$ (82\%). Mp $182{ }^{\circ} \mathrm{C} .{ }^{1} \mathrm{H}$ NMR $\left(500 \mathrm{MHz}, \mathrm{C}_{6} \mathrm{D}_{6}\right): \delta 6.74-7.28(\mathrm{~m}, 11 \mathrm{H}, \mathrm{Ar}-H), 4.73(\mathrm{q}, 1 \mathrm{H}, \mathrm{CH}), 4.65(\mathrm{~s}, 1 \mathrm{H}, \gamma-$ $\mathrm{CH}$ ), 3.73 (sept, 2H, $\left.\mathrm{CH}\left(\mathrm{CH}_{3}\right)_{2}\right), 3.29$ (sept, 1H, $\left.\mathrm{CH}\left(\mathrm{CH}_{3}\right)_{2}\right), 3.07$ (sept, 1H, $\left.\mathrm{CH}\left(\mathrm{CH}_{3}\right)_{2}\right), 1.60(\mathrm{~d}, 3 \mathrm{H}$, $\left.\mathrm{CH}\left(\mathrm{CH}_{3}\right)_{2}\right), 1.50\left(\mathrm{~d}, 3 \mathrm{H}, \mathrm{CH}\left(\mathrm{CH}_{3}\right)_{2}\right), 1.48\left(\mathrm{~s}, 3 \mathrm{H}, \mathrm{CH}_{3}\right), 1.41\left(\mathrm{~s}, 3 \mathrm{H}, \mathrm{CH}_{3}\right), 1.35\left(\mathrm{~d}, 3 \mathrm{H}, \mathrm{CH}\left(\mathrm{CH}_{3}\right)_{2}\right), 1.21(\mathrm{~d}, 3 \mathrm{H}$, $\left.\mathrm{CH}\left(\mathrm{CH}_{3}\right)_{2}\right), 1.15\left(\mathrm{~d}, 3 \mathrm{H}, \mathrm{CH}\left(\mathrm{CH}_{3}\right)_{2}\right), 1.11$ (d, 3H, $\left.\mathrm{CH}\left(\mathrm{CH}_{3}\right)_{2}\right), 1.02$ (d, 3H, $\left.\mathrm{CH}\left(\mathrm{CH}_{3}\right)_{2}\right), 0.72\left(\mathrm{~d}, 3 \mathrm{H}, \mathrm{CH}(\mathrm{CH})_{2}\right)$ ppm. ${ }^{19} \mathrm{~F}\left\{{ }^{1} \mathrm{H}\right\}$ NMR $(188.29 \mathrm{MHz}): \delta-75.76\left(\mathrm{~d}, 3 \mathrm{~F}, \mathrm{CF} 3,{ }^{3} \mathrm{~J}\left({ }^{19} \mathrm{~F}-{ }^{1} \mathrm{H}\right)=8 \mathrm{~Hz}\right) \mathrm{ppm}$. EI-MS $(70 \mathrm{eV}): m / z(\%): 666$ (100) $[\mathrm{M}]^{+}$. Anal. calcd for $\mathrm{C}_{37} \mathrm{H}_{47} \mathrm{~F}_{3} \mathrm{GeN}_{2} \mathrm{O}$ (665.36): C 66.78, H 7.12, N 4.21; Found: C 66.65, H 7.24, N 4.12.

11.4.8. Synthesis of $\mathrm{LGeOCH}\left(2-\mathrm{C}_{4} \mathrm{H}_{3} \mathrm{~S}\right)\left(\mathrm{CF}_{3}\right)(\mathbf{8})$

A solution of 2,2,2-trifluoro thiophenone $(0.175 \mathrm{~g}, 1.00 \mathrm{mmol}$ in $5 \mathrm{~mL}$ toluene) was added by cannula to a solution of $5(0.49 \mathrm{~g}, 1.00 \mathrm{mmol}$ in toluene $20 \mathrm{~mL})$ at room temperature. After $12 \mathrm{~h}$ all volatiles were removed in vacuo, and the remaining residue was extracted with $n$-hexane $(25 \mathrm{~mL})$. The extract was concentrated to about $15 \mathrm{~mL}$ and stored in a $-30{ }^{\circ} \mathrm{C}$ freezer. Yellow crystals of 8 suitable for X-ray diffraction analysis had formed after two days. Yield: $0.570 \mathrm{~g}(85 \%)$. Mp $171{ }^{\circ} \mathrm{C} .{ }^{1} \mathrm{H} \mathrm{NMR}\left(500 \mathrm{MHz}, \mathrm{C}_{6} \mathrm{D}_{6}\right): \delta 6.93-7.12(\mathrm{~m}, 6 \mathrm{H}, \mathrm{Ar}-$ $H), 6.67\left(\mathrm{~d}, 1 \mathrm{H}, \mathrm{C}_{4} H_{3} \mathrm{~S}\right), 6.45\left(\mathrm{dd}, 1 \mathrm{H}, \mathrm{C}_{4} H_{3} \mathrm{~S}\right), 6.26\left(\mathrm{~d}, 1 \mathrm{H}, \mathrm{C}_{4} H_{3} \mathrm{~S}\right), 5.04(\mathrm{q}, 1 \mathrm{H}, \mathrm{CH}), 4.66(\mathrm{~s}, 1 \mathrm{H}, \gamma-\mathrm{CH}), 3.69$ (sept, 1H, $\left.\mathrm{CH}\left(\mathrm{CH}_{3}\right)_{2}\right), 3.63$ (sept, 1H, $\left.\mathrm{CH}\left(\mathrm{CH}_{3}\right)_{2}\right), 3.28$ (sept, 1H, $\left.\mathrm{CH}\left(\mathrm{CH}_{3}\right)_{2}\right), 3.15$ (sept, 1H, $\left.\mathrm{CH}\left(\mathrm{CH}_{3}\right)_{2}\right), 1.58$ $\left(\mathrm{d}, 3 \mathrm{H}, \mathrm{CH}\left(\mathrm{CH}_{3}\right)_{2}\right), 1.48$ (s, 3H, $\left.\mathrm{CH}_{3}\right), 1.42\left(\mathrm{~s}, 3 \mathrm{H}, \mathrm{CH}_{3}\right), 1.41\left(\mathrm{~d}, 3 \mathrm{H}, \mathrm{CH}\left(\mathrm{CH}_{3}\right)_{2}\right), 1.32$ (d, 3H, $\left.\mathrm{CH}\left(\mathrm{CH}_{3}\right)_{2}\right), 1.20-$ $1.14\left(\mathrm{~m}, 6 \mathrm{H}, \mathrm{CH}\left(\mathrm{CH}_{3}\right)_{2}\right), 1.12\left(\mathrm{~d}, 3 \mathrm{H}, \mathrm{CH}\left(\mathrm{CH}_{3}\right)_{2}\right), 1.07$ (d, 3H, $\left.\mathrm{CH}\left(\mathrm{CH}_{3}\right)_{2}\right), 0.98\left(\mathrm{~d}, 3 \mathrm{H}, \mathrm{CH}\left(\mathrm{CH}_{3}\right)_{2}\right)$ ppm. ${ }^{19} \mathrm{~F}\left\{{ }^{1} \mathrm{H}\right\}$ NMR (188.29 MHz): $\delta-76.29\left(\mathrm{~d}, 3 \mathrm{~F}, \mathrm{CF} 3,{ }^{3} J\left({ }^{19} \mathrm{~F}-{ }^{1} \mathrm{H}\right)=7 \mathrm{~Hz}\right) \mathrm{ppm}$. EI-MS $(70 \mathrm{eV}): m / z(\%): 672(100)$ $[\mathrm{M}]^{+}$. Anal. calcd for $\mathrm{C}_{35} \mathrm{H}_{45} \mathrm{~F}_{3} \mathrm{GeN}_{2} \mathrm{OS}$ (671.38): C 62.61, H 6.76, N 4.17, S 4.78; Found: Found: $\mathrm{C} 62.58, \mathrm{H}$ $6.68, \mathrm{~N} 4.17$, S 5.33 .

\subsubsection{Synthesis of $\mathrm{LGeC}\left(\mathrm{CO}_{2} \mathrm{Me}\right) \mathrm{CH}_{2}(\mathbf{9})$}

A solution of $\mathrm{HC} \equiv \mathrm{CCO}_{2} \mathrm{Me}(0.085 \mathrm{~g}, 1.00 \mathrm{mmol}$ in $5 \mathrm{~mL}$ toluene $)$ was added drop by drop by cannula to a solution of $5(0.490 \mathrm{~g}, 1.00 \mathrm{mmol}$ in toluene $15 \mathrm{~mL})$ at room temperature. After overnight constant stirring at ambient temperature all volatiles were removed in vacuo, and the remaining residue was extracted with $n$ hexane $(15 \mathrm{~mL})$ and after removal of all the volatiles compound 9 was obtained as a yellow powder. Yield: 0.49 $\mathrm{g}(85 \%)$. Mp $161{ }^{\circ} \mathrm{C} .{ }^{1} \mathrm{H}$ NMR ( $\left.500 \mathrm{MHz}, \mathrm{C}_{6} \mathrm{D}_{6}\right): \delta 7.09-7.16(\mathrm{~m}, 6 \mathrm{H}, \mathrm{Ar}-H), 6.20$ (br, $\left.1 \mathrm{H}, \mathrm{CH}_{2}\right), 5.82(\mathrm{br}, 1 \mathrm{H}$, $\left.\mathrm{CH}_{2}\right), 4.88$ (s, $\left.1 \mathrm{H}, \gamma-\mathrm{CH}\right), 3.70$ (sept, $\left.2 \mathrm{H}, \mathrm{CH}\left(\mathrm{CH}_{3}\right)_{2}\right), 3.47$ (s, 3H, $\left.\mathrm{CO}_{2} \mathrm{CH}_{3}\right), 3.46\left(\mathrm{sept}, 2 \mathrm{H}, \mathrm{CH}\left(\mathrm{CH}_{3}\right)_{2}\right), 1.58(\mathrm{~s}$, $\left.6 \mathrm{H}, \mathrm{CH}_{3}\right), 1.31$ (d, 6H, $\left.\mathrm{CH}\left(\mathrm{CH}_{3}\right)_{2}\right), 1.27\left(\mathrm{~d}, 6 \mathrm{H}, \mathrm{CH}\left(\mathrm{CH}_{3}\right)_{2}\right), 1.18\left(\mathrm{~d}, 6 \mathrm{H}, \mathrm{CH}\left(\mathrm{CH}_{3}\right)_{2}\right), 1.15\left(\mathrm{~d}, 6 \mathrm{H}, \mathrm{CH}(\mathrm{CH})_{2}\right)$ ppm. EI-MS: $m / z(\%) 576(100)[\mathrm{M}]^{+}$. Anal. calcd for $\mathrm{C}_{33} \mathrm{H}_{46} \mathrm{GeN}_{2} \mathrm{O}_{2}$ (576): C 68.89, H, 8.06, N 4.87; Found: C, $68.89 ; \mathrm{H}, 8.16 ; \mathrm{N}, 4.20$.

11.4.10. Synthesis of $\mathrm{LGeC}\left(\mathrm{CO}_{2} \mathrm{Et}\right) \mathrm{CH}_{2}(\mathbf{1 0})$

A solution of $\mathrm{HC} \equiv \mathrm{CCO}_{2} \mathrm{Et}(0.100 \mathrm{~g}, 1.00 \mathrm{mmol}$ in $5 \mathrm{~mL}$ toluene $)$ was added drop by drop by cannula to a solution of $5(0.490 \mathrm{~g}, 1.00 \mathrm{mmol}$ in toluene $15 \mathrm{~mL})$ at room temperature. After $0.5 \mathrm{~h}$ under constant stirring at ambient temperature the red solution turned deep-red. All volatiles were removed in vacuo, and the remaining residue was extracted with $n$-hexane $(15 \mathrm{~mL})$ and concentrated to about $5 \mathrm{~mL}$ and stored in a $-30{ }^{\circ} \mathrm{C}$ freezer. Red crystals of 10 suitable for X-ray diffraction analysis are formed after two days. Yield: $0.50 \mathrm{~g}(85 \%)$. Mp 
$170{ }^{\circ} \mathrm{C} .{ }^{1} \mathrm{H}$ NMR ( $\left.500 \mathrm{MHz}, \mathrm{C}_{6} \mathrm{D}_{6}\right): \delta 7.09-7.16(\mathrm{~m}, 6 \mathrm{H}, \mathrm{Ar}-H), 6.20(\mathrm{~d}, 1 \mathrm{H}),, 5.82(\mathrm{~d}, 1 \mathrm{H}),, 4.89(\mathrm{~s}, 1 \mathrm{H}, \gamma-$ $\mathrm{CH}$ ), 4.07 (q, 2H, $\mathrm{CH}_{2}$ ), 3.65 (sept, 2H, $\left.\mathrm{CH}\left(\mathrm{CH}_{3}\right)_{2}\right), 3.48$ (sept, $\left.2 \mathrm{H}, \mathrm{CH}\left(\mathrm{CH}_{3}\right)_{2}\right), 1.59$ (s, $\left.6 \mathrm{H}, \mathrm{CH}_{3}\right), 1.31$ (d, $6 \mathrm{H}$, $\left.\mathrm{CH}\left(\mathrm{CH}_{3}\right)_{2}\right), 1.27\left(\mathrm{~d}, 6 \mathrm{H}, \mathrm{CH}\left(\mathrm{CH}_{3}\right)_{2}\right), 1.18\left(\mathrm{~d}, 6 \mathrm{H}, \mathrm{CH}\left(\mathrm{CH}_{3}\right)_{2}\right), 1.15$ (d, 6H, $\left.\mathrm{CH}\left(\mathrm{CH}_{3}\right)_{2}\right), 0.87\left(\mathrm{t}, 3 \mathrm{H}, \mathrm{CH}_{2} \mathrm{CH}_{3}\right)$ ppm. IR (Nujol, KBr): $\widetilde{v}=2854(\mathrm{C}-\mathrm{H}), 1690 \mathrm{~cm}^{-1}(\mathrm{C}=\mathrm{O})$. EI-MS: $\mathrm{m} / z(\%) 590(100)[\mathrm{M}]^{+}$. Anal. calcd for $\mathrm{C}_{34} \mathrm{H}_{48} \mathrm{GeN}_{2} \mathrm{O}_{2}$ (589.40): C 69.29, H 8.21, N 4.75; Found: C 68.25, H 9.83, N 4.53.

\subsubsection{Synthesis of $\mathrm{LGeC}\left(\mathrm{CO}_{2} \mathrm{Me}\right) \mathrm{CHCO}_{2} \mathrm{Me}(\mathbf{1 1})$}

A solution of dimethyl acetylenedicarboxylate $(0.145 \mathrm{~g}, 1.00 \mathrm{mmol}$ in $5 \mathrm{~mL})$ toluene was added drop by drop by cannula to a solution of $5(0.490 \mathrm{~g}, 1.00 \mathrm{mmol}$ in toluene $15 \mathrm{~mL})$ at room temperature. After $15 \mathrm{~min}$ under constant stirring at ambient temperature the red solution turned deep-red. All volatiles were removed in vacuo, and the remaining residue was extracted with $n$-hexane $(15 \mathrm{~mL})$ and concentrated to about $5 \mathrm{~mL}$ and stored in a $-30{ }^{\circ} \mathrm{C}$ freezer. Red crystals of $\mathbf{1 1}$ suitable for X-ray diffraction analysis are formed after one day. Yield: $0.510 \mathrm{~g}$ (80 \%); Mp $162{ }^{\circ} \mathrm{C} .{ }^{1} \mathrm{H}$ NMR ( $\left.500 \mathrm{MHz}, \mathrm{C}_{6} \mathrm{D}_{6}\right): \delta 7.05-7.15(\mathrm{~m}, 6 \mathrm{H}, \mathrm{Ar}-H), 6.64(\mathrm{~s}, 1 \mathrm{H}, \mathrm{CH}), 4.89(\mathrm{~s}, 1 \mathrm{H}, \gamma-$ $\mathrm{CH}$ ), 3.90 (sept, $\left.2 \mathrm{H}, \mathrm{CH}\left(\mathrm{CH}_{3}\right)_{2}\right), 3.43$ (s, 3H, $\left.\mathrm{CH}_{3}\right), 3.33$ (sept, $\left.2 \mathrm{H}, \mathrm{CH}\left(\mathrm{CH}_{3}\right)_{2}\right), 3.16$ (s, $\left.3 \mathrm{H}, \mathrm{CH}_{3}\right), 1.59$ (s, $6 \mathrm{H}$, $\left.\mathrm{CH}_{3}\right), 1.32$ (d, 6H, $\left.\mathrm{CH}\left(\mathrm{CH}_{3}\right)_{2}\right), 1.24$ (q, $\left.12 \mathrm{H}, \mathrm{CH}\left(\mathrm{CH}_{3}\right)_{2}\right), 1.12$ (d, 6H, $\left.\mathrm{CH}\left(\mathrm{CH}_{3}\right)_{2}\right)$ ppm. EI-MS: $\mathrm{m} / \mathrm{z}(\%) 634$ (100) $[\mathrm{M}]^{+}$. Anal. calcd for $\mathrm{C}_{37} \mathrm{H}_{52} \mathrm{GeN}_{2} \mathrm{O}_{4}$ (633.41): C 66.37, H 7.64, N 4.42; Found: C 66.21, H 7.64, N 4.38.

\subsubsection{Synthesis of $\mathrm{LGeC}\left(\mathrm{CO}_{2} \mathrm{Et}\right) \mathrm{CHCO}_{2} \mathrm{Et}(\mathbf{1 2})$}

A solution of diethyl acetylenedicarboxylate $(0.170 \mathrm{~g}, 1.00 \mathrm{mmol}$ in $5 \mathrm{~mL}$ toluene) was added drop by drop by cannula to a solution of $5(0.490 \mathrm{~g}, 1.00 \mathrm{mmol}$ in toluene $15 \mathrm{~mL})$ at room temperature. After $24 \mathrm{~h}$ under constant stirring at ambient temperature the red solution turned yellow. All volatiles were removed in vacuo, and the remaining residue was extracted with $n$-hexane $(25 \mathrm{~mL})$ and concentrated to about $15 \mathrm{~mL}$ and stored in a $-30{ }^{\circ} \mathrm{C}$ freezer. Yellow crystals of $\mathbf{1 2}$ suitable for X-ray diffraction analysis are formed after two days. Yield: $0.53 \mathrm{~g}$ (80 \%). Mp $117^{\circ} \mathrm{C} .{ }^{1} \mathrm{H}$ NMR ( $\left.500 \mathrm{MHz}, \mathrm{C}_{6} \mathrm{D}_{6}\right): \delta 7.07-7.15(\mathrm{~m}, 6 \mathrm{H}, \mathrm{Ar}-H), 6.68(\mathrm{~s}, 1 \mathrm{H}, \mathrm{CH}), 4.91(\mathrm{~s}, 1 \mathrm{H}, \gamma-$ $\mathrm{CH}$ ), 3.99 (q, 2H, $\mathrm{CH}_{2}$ ), 3.91 (sept, $\left.2 \mathrm{H}, \mathrm{CH}\left(\mathrm{CH}_{3}\right)_{2}\right), 3.82$ (q, 2H, $\mathrm{CH}_{2}$ ), 3.34 (sept, $\left.2 \mathrm{H}, \mathrm{CH}\left(\mathrm{CH}_{3}\right)_{2}\right), 1.63$ (s, $6 \mathrm{H}$, $\left.\mathrm{CH}_{3}\right), 1.34\left(\mathrm{~d}, 6 \mathrm{H}, \mathrm{CH}\left(\mathrm{CH}_{3}\right)_{2}\right), 1.29\left(\mathrm{~d}, 6 \mathrm{H}, \mathrm{CH}\left(\mathrm{CH}_{3}\right)_{2}\right), 1.21\left(\mathrm{~d}, 6 \mathrm{H}, \mathrm{CH}\left(\mathrm{CH}_{3}\right)_{2}\right), 1.14\left(\mathrm{~d}, 6 \mathrm{H}, \mathrm{CH}\left(\mathrm{CH}_{3}\right)_{2}\right), 1.05$ (t, $3 \mathrm{H}, \mathrm{CH}_{2} \mathrm{CH}_{3}$ ), 0.74 (t, 3H, $\mathrm{CH}_{2} \mathrm{CH}_{3}$ ) ppm. EI-MS: $m / z$ (\%) 633 (100) [M-Et] $]^{+}$. Anal. calcd for $\mathrm{C}_{37} \mathrm{H}_{52} \mathrm{GeN}_{2} \mathrm{O}_{4}(661.40):$ C 69.29, H 8.21, N 4.75; Found: C 68.25, H 9.83, N 4.53.

\subsubsection{Synthesis of $\mathrm{LGeC}\left(\mathrm{CO}_{2} t \mathrm{Bu}\right) \mathrm{CHCO}_{2} t \mathrm{Bu}(\mathbf{1 3})$}

A solution of ditertbutyl acetylenedicarboxylate $(0.230 \mathrm{~g}, 1.00 \mathrm{mmol}$ in $5 \mathrm{~mL}$ toluene) was added drop by drop by cannula to a solution of $\mathbf{5}(0.490 \mathrm{~g}, 1.00 \mathrm{mmol}$ in toluene $15 \mathrm{~mL})$ at room temperature. After $45 \mathrm{~min}$ under constant stirring at ambient temperature the red solution turned yellow. All volatiles were removed in vacuo and the remaining residue was extracted with $n$-hexane $(15 \mathrm{~mL})$ and concentrated to about $5 \mathrm{~mL}$ and stored in a -30 ${ }^{\circ} \mathrm{C}$ freezer. Red crystals of $\mathbf{1 3}$ suitable for X-ray diffraction analysis are formed after two days. Yield: $0.630 \mathrm{~g}$ (88 \%). Mp $168^{\circ} \mathrm{C} .{ }^{1} \mathrm{H}$ NMR ( $\left.500 \mathrm{MHz}, \mathrm{C}_{6} \mathrm{D}_{6}\right): \delta 7.04-7.15$ (m, 6H, Ar- $H$ ), $6.65(\mathrm{~s}, 1 \mathrm{H}, \mathrm{CH}), 4.96(\mathrm{~s}, 1 \mathrm{H}, \gamma-$ $\mathrm{CH}$ ), 3.77 (sept, 2H, $\left.\mathrm{CH}\left(\mathrm{CH}_{3}\right)_{2}\right), 3.38$ (sept, 2H, $\left.\mathrm{CH}\left(\mathrm{CH}_{3}\right)_{2}\right), 1.67$ (s, 6H, $\left.\mathrm{CH} 3\right), 1.41$ (s, 9H, $\left.\mathrm{C}\left(\mathrm{CH}_{3}\right)_{3}\right), 1.34$ (d, $\left.6 \mathrm{H}, \mathrm{CH}\left(\mathrm{CH}_{3}\right)_{2}\right), 1.32$ (d, 6H, $\left.\mathrm{CH}\left(\mathrm{CH}_{3}\right)_{2}\right), 1.28\left(\mathrm{~d}, 6 \mathrm{H}, \mathrm{CH}\left(\mathrm{CH}_{3}\right)_{2}\right) 1.18$ (s, 9H, $\left.\mathrm{C}\left(\mathrm{CH}_{3}\right)_{3}\right), 1.15$ (d, 6H, $\left.\mathrm{CH}\left(\mathrm{CH}_{3}\right)_{2}\right)$ ppm. EI-MS: $m / z$ (\%) 718 (100) [M] ${ }^{+}$. Anal. calcd for $\mathrm{C}_{37} \mathrm{H}_{52} \mathrm{GeN}_{2} \mathrm{O}_{4}$ (717.57): C 68.63, H 8.43, N 3.90; Found: C $68.83, \mathrm{H} 8.26, \mathrm{~N} 3.88$.

\subsubsection{Synthesis of $\mathrm{LGe}\left(\mathrm{NHCO}_{2} \mathrm{Et}\right) \mathrm{NCO}_{2} \mathrm{Et}(\mathbf{1 4})$}

A solution of diethyl diazadicarboxylate $(0.145 \mathrm{~g}, 1.00 \mathrm{mmol}$ in $5 \mathrm{~mL})$ toluene was added drop by drop by cannula to a solution of $5(0.490 \mathrm{~g}, 1.00 \mathrm{mmol}$ in toluene $15 \mathrm{~mL})$ at room temperature. After $15 \mathrm{~min}$ under constant stirring at ambient temperature the red solution turned yellow. All volatiles were removed in vacuo, and the remaining residue was extracted with $n$-hexane $(15 \mathrm{~mL})$ and concentrated to about $5 \mathrm{~mL}$ and stored in a $-30{ }^{\circ} \mathrm{C}$ freezer. Yellow crystals of $\mathbf{1 4}$ suitable for X-ray diffraction analysis are formed after three days. Yield: $1.10 \mathrm{~g}(95 \%)$. Mp $162{ }^{\circ} \mathrm{C} .{ }^{1} \mathrm{H}$ NMR $\left(500 \mathrm{MHz}, \mathrm{C}_{6} \mathrm{D}_{6}\right): \delta 7.03-7.16$ (m, 6H, Ar- $H$ ), $6.43(\mathrm{br}, 1 \mathrm{H}, \mathrm{N} H), 5.14(\mathrm{~s}$, $1 \mathrm{H}, \gamma-\mathrm{CH}), 4.00$ (q, 2H, $\left.\mathrm{CH}_{2} \mathrm{CH}_{3}\right), 3.91$ (sept, $\left.2 \mathrm{H}, \mathrm{CH}\left(\mathrm{CH}_{3}\right)_{2}\right), 3.13$ (sept, $\left.2 \mathrm{H}, \mathrm{CH}\left(\mathrm{CH}_{3}\right)_{2}\right), 1.65$ (s, $6 \mathrm{H}, \mathrm{CH}_{3}$ ), $1.36\left(\mathrm{~d}, 6 \mathrm{H}, \mathrm{CH}\left(\mathrm{CH}_{3}\right)_{2}\right), 1.25\left(\mathrm{~d}, 6 \mathrm{H}, \mathrm{CH}\left(\mathrm{CH}_{3}\right)_{2}\right), 1.15\left(\mathrm{~d}, 6 \mathrm{H}, \mathrm{CH}\left(\mathrm{CH}_{3}\right)_{2}\right), 1.14\left(\mathrm{~d}, 6 \mathrm{H}, \mathrm{CH}\left(\mathrm{CH}_{3}\right)_{2}\right), 0.88(\mathrm{t}, 3 \mathrm{H}$, $\mathrm{CH}_{2} \mathrm{CH}_{3}$ ) ppm. EI-MS (70 eV): $\mathrm{m} / z(\%): 491(100)\left[\mathrm{M}-\mathrm{N}_{2} \mathrm{C}_{6} \mathrm{H}_{11} \mathrm{O}_{4}\right]^{+}$. Anal. calcd for $\mathrm{C}_{35} \mathrm{H}_{52} \mathrm{GeN}_{4} \mathrm{O}_{4}(665.45): \mathrm{C}$ 63.17, H 7.88, N 8.42; found: C 61.63, H 7.93, N 8.46. 
11.4.15. Synthesis of $\mathrm{LGeOH}(\mathbf{1 5})$

Method A: Dry $\mathrm{N}_{2} \mathrm{O}$ was bubbled into a solution of $5(0.49 \mathrm{~g}, 1.00 \mathrm{mmol})$ in toluene $(20 \mathrm{~mL})$ at room temperature. After 30 min the gas flow of $\mathrm{N}_{2} \mathrm{O}$ was disconnected, and all the volatiles were removed in vacuo. The residue was treated with $n$-hexane $(40 \mathrm{~mL})$ and after filtration and drying in vacuo, 15 was obtained as a yellow microcrystalline powder. Yield: $0.480 \mathrm{~g}(95 \%)$. Method B: Water $(18 \mu \mathrm{l}, 1 \mathrm{mmol})$ was added to a red solution of $19(0.490 \mathrm{~g}, 1 \mathrm{mmol})$ in toluene $(20 \mathrm{~mL})$ under stirring at room temperature. The reaction mixture became yellow. Stirring of the reaction mixture was continued for about $15 \mathrm{~min}$. After that the solvent was removed in vacuo and the residue was extracted with $n$-hexane $(15 \mathrm{~mL})$. The solution was reduced to half of the volume. Storage of the solution at $-30{ }^{\circ} \mathrm{C}$ for one day yielded yellow crystals of 15 . Yield: $0.480 \mathrm{~g}(95 \%),{ }^{1} \mathrm{H}$ NMR (500 MHz, $\left.\mathrm{C}_{6} \mathrm{D}_{6}\right): \delta 7.09-7.17$ (m, 6H, Ar- $H$ ), 4.91 (s, $1 \mathrm{H}, \gamma-\mathrm{CH}$ ), 3.71 (sept, 2H, $\left.\mathrm{CH}\left(\mathrm{CH}_{3}\right)_{2}\right), 3.31$ (sept, $\left.2 \mathrm{H}, \mathrm{CH}\left(\mathrm{CH}_{3}\right)_{2}\right), 1.65(\mathrm{~s}, 1 \mathrm{H}, \mathrm{OH}), 1.60\left(\mathrm{~s}, 6 \mathrm{H}, \mathrm{CH}_{3}\right), 1.30$ (d, 6H, $\left.\mathrm{CH}\left(\mathrm{CH}_{3}\right)_{2}\right), 1.28$ (d, $\left.6 \mathrm{H}, \mathrm{CH}\left(\mathrm{CH}_{3}\right)_{2}\right), 1.20$ (d, $\left.6 \mathrm{H}, \mathrm{CH}\left(\mathrm{CH}_{3}\right)_{2}\right), 1.11\left(\mathrm{~d}, 6 \mathrm{H}, \mathrm{CH}\left(\mathrm{CH}_{3}\right)_{2}\right) \mathrm{ppm}$.

\subsubsection{Synthesis of LGe(S)SH (16)}

A solution of $5(1.47 \mathrm{~g}, 3.00 \mathrm{mmol})$ in toluene $(30 \mathrm{~mL})$ was slowly added to a suspension of elemental sulfur $(0.192 \mathrm{~g}, 6.00 \mathrm{mmol})$ in toluene $(15 \mathrm{~mL})$ by cannula at room temperature. After $0.5 \mathrm{~h}$ under constant stirring at ambient temperature the red solution turned slightly yellow. All volatiles were removed in vacuo, and the remaining residue was extracted with toluene $(15 \mathrm{~mL})$. The solvent was removed in vacuo to yield $\mathbf{1 6}$ as a slight yellow powder. Yield: $0.980 \mathrm{~g}(60 \%)$. Mp $220{ }^{\circ} \mathrm{C}$ (decomp). ${ }^{1} \mathrm{H}$ NMR $\left(500 \mathrm{MHz}, \mathrm{C}_{6} \mathrm{D}_{6}\right): \delta 6.99-7.14(\mathrm{~m}, 6 \mathrm{H}$, Ar- $H$ ), 4.83 (s, $1 \mathrm{H}, \gamma-\mathrm{CH}$ ), 3.56 (sept, $\left.2 \mathrm{H}, \mathrm{CH}\left(\mathrm{CH}_{3}\right)_{2}\right), 3.33$ (sept, 2H, $\left.\mathrm{CH}\left(\mathrm{CH}_{3}\right)_{2}\right), 1.72$ (s, 6H, $\left.\mathrm{CH}_{3}\right), 1.65$ (d, $\left.6 \mathrm{H}, \mathrm{CH}\left(\mathrm{CH}_{3}\right)_{2}\right), 1.49$ (d, 12H, $\left.\mathrm{CH}\left(\mathrm{CH}_{3}\right)_{2}\right), 1.13$ (d, 6H, $\left.\mathrm{CH}\left(\mathrm{CH}_{3}\right)_{2}\right),-0.094$ (s, 1H, SH) ppm. IR (Nujol, $\mathrm{KBr}$ ): $\widetilde{v}=2590 \mathrm{~cm}^{-1}(\mathrm{~S}-\mathrm{H})$. EI-MS (70 eV): $m / z(\%): 522(40)[\mathrm{M}-\mathrm{SH}]^{+}, 506(100)$ [M-SH, $\left.-\mathrm{Me}\right]^{+}$. Anal. calcd for $\mathrm{C}_{29} \mathrm{H}_{42} \mathrm{GeN}_{2} \mathrm{~S}_{2}$ (552.25): C 62.71, H 7.62 N 5.04; Found: C 61.26, H 7.70, N 4.89.

\subsubsection{Synthesis of $\mathrm{LGeN}_{3}(\mathbf{1 7})$ and $\mathrm{L}^{\prime} \mathrm{Ge}\left(\mathrm{NHSiMe}_{3}\right)_{2}(\mathbf{1 8})$}

An excess of trimethylsilyl azide was slowly added drop by drop to a $20 \mathrm{~mL}$ toluene solution of $\mathbf{5}(0.98 \mathrm{~g}, 2$ $\mathrm{mmol}$ ). Then the reaction mixture was stored overnight. After that the solvent and excess trimethylsilyl azide were removed in vacuo. The residue was extracted with $n$-hexane $(35 \mathrm{~mL})$ and the extract concentrated to about $20 \mathrm{~mL}$. After one day at room temperature colorless crystals of $\mathbf{1 7}$ are formed. Then the residual extract is stored in a freezer at $-30{ }^{\circ} \mathrm{C}$. After two days colorless crystals of $18 \mathrm{had}$ formed. 17: Yield: $0.34 \mathrm{~g}(32 \%)$. Mp $190{ }^{\circ} \mathrm{C}$. ${ }^{1} \mathrm{H}$ NMR (500 MHz, $\left.\mathrm{C}_{6} \mathrm{D}_{6}\right): \delta 7.04-7.13(\mathrm{~m}, 6 \mathrm{H}, \mathrm{Ar}-\mathrm{H}$ ), 4.98 (s, $1 \mathrm{H}, \gamma-\mathrm{CH}), 3.73$ (sept, $\left.2 \mathrm{H}, \mathrm{CH}\left(\mathrm{CH}_{3}\right)_{2}\right), 3.10$ (sept, 2H, $\left.\mathrm{CH}\left(\mathrm{CH}_{3}\right)_{2}\right), 1.53$ (s, 6H, $\left.\mathrm{CH}_{3}\right), 1.32$ (d, 6H, $\left.\mathrm{CH}\left(\mathrm{CH}_{3}\right)_{2}\right), 1.18$ (d, 12H, $\left.\mathrm{CH}\left(\mathrm{CH}_{3}\right)_{2}\right), 1.15$ (d, 6H, $\left.\mathrm{CH}\left(\mathrm{CH}_{3}\right)_{2}\right), 1.05$ (d, 6H, $\left.\mathrm{CH}\left(\mathrm{CH}_{3}\right)_{2}\right)$ ppm. IR (Nujol, $\left.\mathrm{KBr}, \mathrm{cm}^{-1}\right): \widetilde{v}=2068\left(\mathrm{~N}_{3}\right)$; EI-MS $(70 \mathrm{eV}): \mathrm{m} / z(\%)$ : 491 (100) $\left[\mathrm{M}-\mathrm{N}_{3}\right]^{+}$. Anal. calcd for $\mathrm{C}_{29} \mathrm{H}_{41} \mathrm{GeN}_{5}$ (532.26): C 65.43, H 7.76; Found: C 64.06, H, 8.11. 18: Yield: $0.37 \mathrm{~g}(28 \%)$. Mp $200{ }^{\circ} \mathrm{C} .{ }^{1} \mathrm{H}$ NMR (500 MHz, $\left.\mathrm{C}_{6} \mathrm{D}_{6}\right): \delta 7.21(\mathrm{~s}, 2 \mathrm{H}, \mathrm{N} H), 7.04-7.13(\mathrm{~m}, 6 \mathrm{H}, \mathrm{Ar}-H), 5.29$ (s, $1 \mathrm{H}, \gamma-\mathrm{CH}$ ), 3.87 (s, $1 \mathrm{H}, \mathrm{CH}$ ), 3.86 (sept, $\left.2 \mathrm{H}, \mathrm{CH}\left(\mathrm{CH}_{3}\right)_{2}\right), 3.74$ (sept, $\left.2 \mathrm{H}, \mathrm{CH}\left(\mathrm{CH}_{3}\right)_{2}\right), 3.28$ (s, $\left.1 \mathrm{H}, \mathrm{CH}\right), 1.58$ (s, 6H, $\left.\mathrm{CH}_{3}\right), 1.32$ (d, 6H, $\left.\mathrm{CH}\left(\mathrm{CH}_{3}\right)_{2}\right), 1.18$ (d, 12H, $\left.\mathrm{CH}\left(\mathrm{CH}_{3}\right)_{2}\right), 1.15$ (d, 6H, $\left.\mathrm{CH}\left(\mathrm{CH}_{3}\right)_{2}\right), 1.05$ (d, 6H, $\left.\mathrm{CH}\left(\mathrm{CH}_{3}\right)_{2}\right)$,

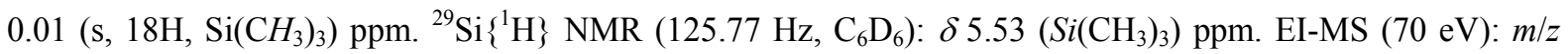
(\%): 651 (100) [M-Me] $]^{+}$. Anal. calcd for $\mathrm{C}_{35} \mathrm{H}_{60} \mathrm{GeN}_{4} \mathrm{Si}_{2}$ (665.64): C 63.15, H 9.08; Found: C 64.83, H 8.36.

\subsubsection{Synthesis of $L^{\prime} G e(19)$}

Method A: A solution of $\mathrm{B}\left(\mathrm{C}_{6} \mathrm{~F}_{5}\right)_{3}(0.51 \mathrm{~g}, 1.0 \mathrm{mmol})$ in toluene $(10 \mathrm{~mL})$ was added drop by drop to a stirred solution of $5(0.49 \mathrm{~g}, 1.0 \mathrm{mmol})$ in toluene $(10 \mathrm{~mL})$ at room temperature. Immediately the red color of the reaction mixture changed to colorless. The reaction mixture was stirred for additional $10 \mathrm{~min}$. After that a solution of 1,3-di-tert-butylimidazol-2-ylidene $(0.18 \mathrm{~g}, 1.0 \mathrm{mmol})$ in toluene $(10 \mathrm{~mL})$ was added drop by drop to the reaction mixture. During this time the reaction mixture turns to brown-red with the formation of a white precipitate. After another $10 \mathrm{~min}$ the solvent was removed in vacuo and the residue was extracted with $n$-hexane $(15 \mathrm{~mL})$. The extracted brown-red solution was identified as 19. The insoluble part was collected and identified as imidazolium borate salt. Both are formed almost quantitatively and the NMR data matches with the previously reported samples. Method B: 1,3-di-tert-butylimidazol-2-ylidene $(0.360 \mathrm{~g}, 2.0 \mathrm{mmol})$ and $\mathrm{LGeCl}$ $(1.050 \mathrm{~g}, 2.0 \mathrm{mmol})$ were dissolved in toluene $(30 \mathrm{~mL})$ at room temperature. The reaction mixture was stirred 
and the color of the solution changed from yellow to brown-red. A white precipitate was formed. The reaction mixture was stirred for another hour, then the white precipitate was separated by filtration and the remaining red solution was evaporated. The residue was dissolved in $n$-hexane. Storage of the solution at $-30^{\circ} \mathrm{C}$ for one day yielded brown-red crystals suitable for a single crystal X-ray diffraction experiment. Yield: $0.630 \mathrm{~g}(65 \%)$. Method C: 1,3-Di-tert-butylimidazol-2-ylidene $(0.360 \mathrm{~g}, 2.0 \mathrm{mmol})$ and LGeOTf $(1.280 \mathrm{~g}, 2.0 \mathrm{mmol})$ were dissolved in toluene $(30 \mathrm{~mL})$ at room temperature. The reaction mixture was stirred and the color of the solution changed from colorless to brown-red. A white precipitate was formed. The reaction mixture was stirred for another hour, then the white precipitate was separated by filtration and the remaining red solution was evaporated. The residue was dissolved in $n$-hexane $(20 \mathrm{~mL})$. Storage of this solution at $-30{ }^{\circ} \mathrm{C}$ for one day in a freezer yielded brown-red crystals. Yield: $0.880 \mathrm{~g}(90 \%) .{ }^{1} \mathrm{H}$ NMR $\left(500 \mathrm{MHz}, \mathrm{C}_{6} \mathrm{D}_{6}\right): \delta 7.04-7.21(\mathrm{~m}, 6 \mathrm{H}, \mathrm{Ar}-\mathrm{H}$ ), 5.43 (s, $1 \mathrm{H}, \gamma-\mathrm{CH}), 3.91$ (s, $\left.1 \mathrm{H}, \mathrm{CH})_{2}\right), 3.69$ (sept, $\left.2 \mathrm{H}, \mathrm{CH}\left(\mathrm{CH}_{3}\right)_{2}\right), 3.44$ (sept, $\left.2 \mathrm{H}, \mathrm{CH}\left(\mathrm{CH}_{3}\right)_{2}\right), 3.26(\mathrm{~s}, 1 \mathrm{H}$, $\left.\mathrm{CH}_{2}\right), 1.41$ (s, 3H, $\left.\mathrm{CH}_{3}\right), 1.35$ (d, 6H, $\left.\mathrm{CH}\left(\mathrm{CH}_{3}\right)_{2}\right), 1.29$ (d, 6H, $\left.\mathrm{CH}\left(\mathrm{CH}_{3}\right)_{2}\right), 1.20$ (d, 6H, $\left.\mathrm{CH}\left(\mathrm{CH}_{3}\right)_{2}\right), 1.15$ (d, 6H, $\left.\mathrm{CH}\left(\mathrm{CH}_{3}\right)_{2}\right) \mathrm{ppm}$.

\subsubsection{Synthesis of LSnH (20)}

A solution of $\mathrm{K}\left[\mathrm{HB}(\sec \mathrm{Bu})_{3}\right]$ in THF $(2.00 \mathrm{~mL}, 1 \mathrm{M}$, in THF) was slowly added drop by drop to a stirred solution of $\mathrm{LSnCl}(1.140 \mathrm{~g}, 2 \mathrm{mmol})$ in toluene $(30 \mathrm{~mL})$ at $-10{ }^{\circ} \mathrm{C}$. The reaction mixture was warmed to room temperature and was stirred for additional $30 \mathrm{~min}$. After removal of all the volatiles, the residue was extracted with $n$-hexane $(30 \mathrm{~mL})$ and concentrated to about $10 \mathrm{~mL}$ and stored in a $-30{ }^{\circ} \mathrm{C}$ freezer. Yellow crystals of 20 are formed after one day. Yield: $0.94 \mathrm{~g}(88 \%)$. Mp $125^{\circ} \mathrm{C} .{ }^{1} \mathrm{H}$ NMR $\left(500 \mathrm{MHz}, \mathrm{C}_{6} \mathrm{D}_{6}\right): \delta 13.83(\mathrm{~s}, 1 \mathrm{H}, \mathrm{Sn}-H)$, 7.15-7.06 (m, 6H, Ar- $H$ ), 4.89 (s, $1 \mathrm{H}, \gamma-\mathrm{CH}$ ), 3.52 (sept, 2H, $\left.\mathrm{CH}\left(\mathrm{CH}_{3}\right)_{2}\right), 3.46$ (sept, $\left.2 \mathrm{H}, \mathrm{CH}\left(\mathrm{CH}_{3}\right)_{2}\right), 1.62$ (s, $\left.6 \mathrm{H}, \mathrm{CH}_{3}\right), 1.34$ (d, 6H, $\left.\mathrm{CH}\left(\mathrm{CH}_{3}\right)_{2}\right), 1.27\left(\mathrm{~d}, 6 \mathrm{H}, \mathrm{CH}\left(\mathrm{CH}_{3}\right)_{2}\right), 1.19$ (d, $\left.12 \mathrm{H}, \mathrm{CH}\left(\mathrm{CH}_{3}\right)_{2}\right), \mathrm{ppm} .{ }^{119} \mathrm{Sn}\left\{{ }^{1} \mathrm{H}\right\} \mathrm{NMR}$ (186.46 MHz): $\delta-4.45 \mathrm{ppm}\left({ }^{2} J\left({ }^{19} \mathrm{Sn},{ }^{1} \mathrm{H}\right)=64 \mathrm{~Hz}\right)$.

\subsubsection{Synthesis of $\mathrm{LSnOC}(\mathrm{O}) \mathrm{H}(\mathbf{2 1})$}

Dry $\mathrm{CO}_{2}$ was bubbled into a solution of $20(1.08 \mathrm{~g}, 2.00 \mathrm{mmol})$ in toluene $(40 \mathrm{~mL})$ at room temperature. Immediately after the addition of $\mathrm{CO}_{2}$, the color of the solution changed to colorless. After $10 \mathrm{~min}$ the gas flow of $\mathrm{CO}_{2}$ was disconnected, and the solution was stirred for additional $30 \mathrm{~min}$. All the volatiles were removed in vасuo. The residue was treated with $n$-hexane $(40 \mathrm{~mL})$ and after filtration and drying in vacuo, 21 was obtained as a yellow microcrystalline powder. Yield $(1.10 \mathrm{~g}, 95 \%)$. Mp $183{ }^{\circ} \mathrm{C} .{ }^{1} \mathrm{H}$ NMR $\left(500 \mathrm{MHz}, \mathrm{C}_{6} \mathrm{D}_{6}\right): \delta 8.97$ (s, $1 \mathrm{H}, \mathrm{CH}$ ), $7.02-7.15$ (m, 6H, Ar- $H$ ), 4.96 (s, $1 \mathrm{H}, \gamma-\mathrm{CH}), 3.61$ (sept, $\left.2 \mathrm{H}, \mathrm{CH}\left(\mathrm{CH}_{3}\right)_{2}\right), 3.06$ (sept, $\left.2 \mathrm{H}, \mathrm{CH}\left(\mathrm{CH}_{3}\right)_{2}\right)$, $1.58\left(\mathrm{~s}, 6 \mathrm{H}, \mathrm{CH}_{3}\right), 1.32$ (d, 6H, $\left.\mathrm{CH}\left(\mathrm{CH}_{3}\right)_{2}\right), 1.18\left(\mathrm{~d}, 12 \mathrm{H}, \mathrm{CH}\left(\mathrm{CH}_{3}\right)_{2}\right), 1.15$ (d, 6H, $\left.\mathrm{CH}\left(\mathrm{CH}_{3}\right)_{2}\right), 1.05$ (d, 6H, $\left.\mathrm{CH}\left(\mathrm{CH}_{3}\right)_{2}\right) \mathrm{ppm} .{ }^{119} \mathrm{Sn}\left\{{ }^{1} \mathrm{H}\right\}$ NMR (186.46 MHz): $\delta-360 \mathrm{ppm}$. IR (Nujol, $\left.\mathrm{KBr}, \mathrm{cm}^{-1}\right): \tilde{v}=2700(\mathrm{C}-\mathrm{H}), 1641$ $(\mathrm{C}=\mathrm{O})$. EI-MS (70 eV): $m / z(\%): 582(100)[\mathrm{M}]^{+}$. Anal. calcd for $\mathrm{C}_{30} \mathrm{H}_{42} \mathrm{~N}_{2} \mathrm{O}_{2} \mathrm{Sn}(582.23)$ : C 61.98, H 7.28, N 4.82; Found: C 61.69, H 7.39, N 4.82 .

\subsubsection{Synthesis of $\mathrm{LSnOCH}_{2} \mathrm{Fc}$ (22)}

A solution of ferrocene carbaldehyde $(0.210 \mathrm{~g}, 1.00 \mathrm{mmol}$ in $5 \mathrm{~mL}$ toluene) was added by cannula to a solution of $20(0.54 \mathrm{~g}, 1.00 \mathrm{mmol}$ in toluene $15 \mathrm{~mL})$ at room temperature under stirring. After $12 \mathrm{~h}$ all volatiles were removed in vacuo, and the remaining residue was extracted with $n$-hexane $(15 \mathrm{~mL})$ and concentrated to about 5 $\mathrm{mL}$ and stored in a $-30{ }^{\circ} \mathrm{C}$ freezer. Red-yellow crystals of $\mathbf{2 2}$ suitable for X-ray diffraction analysis are formed after one day. Yield: $0.635 \mathrm{~g}(85 \%)$. Mp $143{ }^{\circ} \mathrm{C} .{ }^{1} \mathrm{H}$ NMR $\left(500 \mathrm{MHz}, \mathrm{C}_{6} \mathrm{D}_{6}\right): \delta 7.06-7.17(\mathrm{~m}, 6 \mathrm{H}, \mathrm{Ar}-H), 4.87$ (s, $1 \mathrm{H}, \gamma-\mathrm{CH}), 4.81\left(\mathrm{~s}, 2 \mathrm{H}, \mathrm{CH}_{2}\right), 4.09\left(\mathrm{t}, 2 \mathrm{H}, \alpha-\mathrm{C}_{5} \mathrm{H}_{4}\right), 4.01\left(\mathrm{~s}, 5 \mathrm{H}, \mathrm{C}_{5} \mathrm{H}_{5}\right), 3.92\left(\mathrm{t}, 2 \mathrm{H}, \beta-\mathrm{C}_{5} \mathrm{H}_{4}\right), 3.83(\mathrm{sept}, 2 \mathrm{H}$, $\left.\mathrm{CH}\left(\mathrm{CH}_{3}\right)_{2}\right), 3.19$ (sept, $\left.2 \mathrm{H}, \mathrm{CH}\left(\mathrm{CH}_{3}\right)_{2}\right), 1.62$ (s, 6H, $\left.\mathrm{CH}_{3}\right), 1.44$ (s, 6H, $\left.\mathrm{CH}_{3}\right), 1.30$ (d, 6H, $\left.\mathrm{CH}\left(\mathrm{CH}_{3}\right)_{2}\right), 1.20$ (d, $\left.6 \mathrm{H}, \mathrm{CH}\left(\mathrm{CH}_{3}\right)_{2}\right), 1.12\left(\mathrm{~d}, 6 \mathrm{H}, \mathrm{CH}\left(\mathrm{CH}_{3}\right)_{2}\right) \mathrm{ppm} .{ }^{119} \mathrm{Sn}\left\{{ }^{1} \mathrm{H}\right\} \mathrm{NMR}(186.46 \mathrm{MHz}): \delta-262 \mathrm{ppm}$. EI-MS $(70 \mathrm{eV}): \mathrm{m} / \mathrm{z}$ (\%): 752 (100) $[\mathrm{M}]^{+}$. Anal. calcd for $\mathrm{C}_{40} \mathrm{H}_{52} \mathrm{FeN}_{2} \mathrm{OSn}$ (752.25): C 63.94, H 6.98, N 3.73; Found: $\mathrm{C}$ 63.76, $\mathrm{H}$ 7.01, N 3.65 .

11.4.22. Synthesis of $\mathrm{LSnOCHPh}_{2}(\mathbf{2 3})$

A solution of $\mathrm{Ph}_{2} \mathrm{CO}(0.180 \mathrm{~g}, 1.00 \mathrm{mmol}$ in $5 \mathrm{~mL}$ toluene $)$ was added by cannula to a solution of $20(0.54 \mathrm{~g}$, $1.00 \mathrm{mmol}$ in toluene $20 \mathrm{~mL}$ ) at room temperature. After $12 \mathrm{~h}$ all volatiles were removed in vacuo, and the 
remaining residue was extracted with $n$-hexane $(25 \mathrm{~mL})$ and concentrated to about $15 \mathrm{~mL}$ and stored in a $-30{ }^{\circ} \mathrm{C}$ freezer. After four days yellow crystals of 23 are formed. Yield: $0.590 \mathrm{~g}(82 \%)$. Mp $76{ }^{\circ} \mathrm{C} .{ }^{1} \mathrm{H} \mathrm{NMR}(500 \mathrm{MHz}$, $\left.\mathrm{C}_{6} \mathrm{D}_{6}\right): \delta 6.86-7.70(\mathrm{~m}, 16 \mathrm{H}, \mathrm{Ar}-H), 5.97(\mathrm{~s}, 1 \mathrm{H}, \mathrm{CH}), 4.80(\mathrm{~s}, 1 \mathrm{H}, \gamma-\mathrm{CH}), 3.67\left(\mathrm{sept}, 2 \mathrm{H}, \mathrm{CH}\left(\mathrm{CH}_{3}\right)_{2}\right), 3.15(\mathrm{sept}$, $\left.2 \mathrm{H}, \mathrm{CH}\left(\mathrm{CH}_{3}\right)_{2}\right), 1.57$ (s, 6H, $\left.\mathrm{CH}_{3}\right), 1.19$ (d, 6H, $\left.\mathrm{CH}\left(\mathrm{CH}_{3}\right)_{2}\right), 1.14$ (d, 6H, $\left.\mathrm{CH}\left(\mathrm{CH}_{3}\right)_{2}\right), 1.12\left(\mathrm{~d}, 6 \mathrm{H}, \mathrm{CH}(\mathrm{CH})_{2}\right)$, 1.07 (d, 6H, $\left.\mathrm{CH}\left(\mathrm{CH}_{3}\right)_{2}\right) .{ }^{119} \mathrm{Sn}\left\{{ }^{1} \mathrm{H}\right\}$ NMR (186.46 MHz): $\delta$-218 ppm. EI-MS (70 eV): $\mathrm{m} / z(\%): 719$ (100) [M] $]^{+}$. Anal. calcd for $\mathrm{C}_{42} \mathrm{H}_{52} \mathrm{~N}_{2} \mathrm{OSn}$ (719.59): C 70.10, H 7.28, N 3.89; Found: C 72.71, H 7.53, N 3.32.

11.4.23. Synthesis of $\mathrm{LSnOCHPh}(2-\mathrm{Py})(\mathbf{2 4})$

A solution of 2-benzol pyridine $(0.180 \mathrm{~g}, 1.00 \mathrm{mmol}$ in $5 \mathrm{~mL}$ toluene) was added by cannula to a solution of 20 $(0.54 \mathrm{~g}, 1.00 \mathrm{mmol}$ in toluene $20 \mathrm{~mL})$ at room temperature. After $12 \mathrm{~h}$ all volatiles were removed in vacuo, and the remaining residue was extracted with $n$-hexane $(25 \mathrm{~mL})$ and concentrated to about $15 \mathrm{~mL}$ and stored in a -30 ${ }^{\circ} \mathrm{C}$ freezer. Yellow crystals of $\mathbf{2 4}$ suitable for X-ray diffraction analysis are formed after four days. Yield: $0.660 \mathrm{~g}(92 \%) . \mathrm{Mp} 180{ }^{\circ} \mathrm{C} .{ }^{1} \mathrm{H}$ NMR $\left(500 \mathrm{MHz}, \mathrm{C}_{6} \mathrm{D}_{6}\right): \delta 6.34-7.15(\mathrm{~m}, 15 \mathrm{H}, \mathrm{Ar}-H), 6.28(\mathrm{~s}, 1 \mathrm{H}, \mathrm{CH}), 4.78(\mathrm{~s}$, $1 \mathrm{H}, \gamma-\mathrm{CH}), 4.02$ (sept, 1H, $\left.\mathrm{CH}\left(\mathrm{CH}_{3}\right)_{2}\right), 3.88$ (sept, 1H, $\left.\mathrm{CH}\left(\mathrm{CH}_{3}\right)_{2}\right), 3.41$ (sept, 1H, $\left.\mathrm{CH}\left(\mathrm{CH}_{3}\right)_{2}\right), 3.34$ (sept, $1 \mathrm{H}$, $\left.\mathrm{CH}\left(\mathrm{CH}_{3}\right)_{2}\right), 1.69\left(\mathrm{~s}, 3 \mathrm{H}, \mathrm{CH}_{3}\right), 1.59\left(\mathrm{~s}, 3 \mathrm{H}, \mathrm{CH}_{3}\right), 1.44\left(\mathrm{~d}, 3 \mathrm{H}, \mathrm{CH}\left(\mathrm{CH}_{3}\right)_{2}\right), 1.39\left(\mathrm{~d}, 3 \mathrm{H}, \mathrm{CH}\left(\mathrm{CH}_{3}\right)_{2}\right), 1.27(\mathrm{~d}, 3 \mathrm{H}$, $\left.\mathrm{CH}\left(\mathrm{CH}_{3}\right)_{2}\right), 1.22\left(\mathrm{~d}, 3 \mathrm{H}, \mathrm{CH}\left(\mathrm{CH}_{3}\right)_{2}\right), 1.16\left(\mathrm{~d}, 3 \mathrm{H}, \mathrm{CH}\left(\mathrm{CH}_{3}\right)_{2}\right), 1.11\left(\mathrm{~d}, 3 \mathrm{H}, \mathrm{CH}\left(\mathrm{CH}_{3}\right)_{2}\right), 1.05\left(\mathrm{~d}, 3 \mathrm{H}, \mathrm{CH}(\mathrm{CH})_{2}\right)$, $0.88\left(\mathrm{~d}, 3 \mathrm{H}, \mathrm{CH}\left(\mathrm{CH}_{3}\right)_{2}\right)$ ppm. ${ }^{119} \mathrm{Sn}\left\{{ }^{1} \mathrm{H}\right\}$ NMR (186.46 MHz): $\delta-286 \mathrm{ppm}$. EI-MS $(70 \mathrm{eV}): m / z(\%): 721(100)$ $[\mathrm{M}]^{+}$. Anal. calcd. for $\mathrm{C}_{41} \mathrm{H}_{51} \mathrm{~N}_{3} \mathrm{OSn}$ (721.31): C 68.34, H 7.13, N 5.83; Found: C 68.13, H 7.25, N 5.80.

11.4.24. Synthesis of $\mathrm{LSnOCH}(2-\mathrm{Py})_{2}(\mathbf{2 5})$

A solution of (2-Py) $)_{2} \mathrm{CO}(0.185 \mathrm{~g}, 1.00 \mathrm{mmol}$ in $5 \mathrm{~mL}$ toluene) was added by cannula to a solution of 20 $(0.54 \mathrm{~g}, 1.00 \mathrm{mmol}$ in toluene $20 \mathrm{~mL})$ at room temperature. After overnight stirring all volatiles were removed in vacuo, and the remaining residue was extracted with $n$-hexane $(25 \mathrm{~mL})$. The solvent from the solution was completely removed and compound 25 was obtained as a powder. Yield: $0.560 \mathrm{~g}(78 \%)$. Mp $160{ }^{\circ} \mathrm{C} .{ }^{1} \mathrm{H} \mathrm{NMR}$ $\left(500 \mathrm{MHz}, \mathrm{C}_{6} \mathrm{D}_{6}\right): \delta$ 6.38-7.60 (m, 14H, Ar- $H$ ), $6.59(\mathrm{~s}, 1 \mathrm{H}, \mathrm{CH}), 4.85(\mathrm{~s}, 1 \mathrm{H}, \gamma-\mathrm{CH}), 3.93$ (sept, 2H, $\left.\mathrm{CH}\left(\mathrm{CH}_{3}\right)_{2}\right), 3.55$ (sept, 2H, $\left.\mathrm{CH}\left(\mathrm{CH}_{3}\right)_{2}\right), 1.72(\mathrm{~s}, 3 \mathrm{H}, \mathrm{CH}), 1.44$ (d, 6H, $\left.\mathrm{CH}\left(\mathrm{CH}_{3}\right)_{2}\right), 1.23\left(\mathrm{~d}, 6 \mathrm{H}, \mathrm{CH}(\mathrm{CH})_{2}\right)$, $1.17\left(\mathrm{~d}, 6 \mathrm{H}, \mathrm{CH}\left(\mathrm{CH}_{3}\right)_{2}\right), 0.90\left(\mathrm{~d}, 6 \mathrm{H}, \mathrm{CH}\left(\mathrm{CH}_{3}\right)_{2}\right) \mathrm{ppm} .{ }^{119} \mathrm{Sn}\left\{{ }^{1} \mathrm{H}\right\}$ NMR (186.46 MHz): $\delta$-324 ppm. EI-MS (70 $\mathrm{eV}$ ): $m / z$ (\%): 722 (100) [M] ${ }^{+}$. Anal. calcd for $\mathrm{C}_{40} \mathrm{H}_{50} \mathrm{~N}_{4} \mathrm{OSn}$ (722.30): C 66.58, H 6.98, N 7.76; Found: C 66.74, H 6.94, N 7.67.

\subsubsection{Synthesis of $\mathrm{LSnOCH}\left(\mathrm{C}_{3} \mathrm{H}_{5}\right)_{2}$ (26)}

A solution of dicyclopropylketone $(0.110 \mathrm{~g}, 1.00 \mathrm{mmol}$ in $5 \mathrm{~mL}$ toluene) was added by cannula to a solution of $20(0.54 \mathrm{~g}, 1.00 \mathrm{mmol}$ in toluene $20 \mathrm{~mL})$ at room temperature. After that the solution was heated under reflux for $12 \mathrm{~h}$. Then all volatiles were removed in vacuo, and the remaining residue was extracted with $n$-hexane $(25 \mathrm{~mL})$. The solution was concentrated and stored in a freezer, after four days yellow crystals of $\mathbf{2 6}$ are formed which are suitable for X-ray structural analysis. Yield: $0.480 \mathrm{~g}(74 \%)$. Mp $169{ }^{\circ} \mathrm{C} .{ }^{1} \mathrm{H} \mathrm{NMR}\left(500 \mathrm{MHz}, \mathrm{C}_{6} \mathrm{D}_{6}\right)$ : $\delta$ 7.06-7.20 (m, $6 \mathrm{H}, \mathrm{Ar}-H$ ), 4.71 (s, 1H, $\gamma-\mathrm{CH}), 3.81$ (sept, 2H, $\left.\mathrm{CH}\left(\mathrm{CH}_{3}\right)_{2}\right), 3.24$ (sept, 2H, $\left.\mathrm{CH}\left(\mathrm{CH}_{3}\right)_{2}\right), 2.47(\mathrm{q}$, $1 \mathrm{H}, \mathrm{CH}), 1.58\left(\mathrm{~s}, 6 \mathrm{H}, \mathrm{CH}_{3}\right), 1.52\left(\mathrm{~s}, 6 \mathrm{H}, \mathrm{CH}_{3}\right), 1.28\left(\mathrm{~d}, 6 \mathrm{H}, \mathrm{CH}\left(\mathrm{CH}_{3}\right)_{2}\right), 1.24\left(\mathrm{~d}, 6 \mathrm{H}, \mathrm{CH}\left(\mathrm{CH}_{3}\right)_{2}\right), 1.14(\mathrm{~d}, 6 \mathrm{H}$, $\left.\mathrm{CH}\left(\mathrm{CH}_{3}\right)_{2}\right), 0.75(\mathrm{~m}, 2 \mathrm{H}, \mathrm{CH}), 0.21\left(\mathrm{~m}, 2 \mathrm{H}, \mathrm{CH}_{2}\right), 0.02\left(\mathrm{~m}, 2 \mathrm{H}, \mathrm{CH}_{2}\right),-0.07\left(\mathrm{~m}, 2 \mathrm{H}, \mathrm{CH}_{2}\right),-0.25\left(\mathrm{~m}, 2 \mathrm{H}, \mathrm{CH}_{2}\right)$ ppm. ${ }^{119} \mathrm{Sn}\left\{{ }^{1} \mathrm{H}\right\}$ NMR (186.46 MHz): $\delta-190.50 \mathrm{ppm}$. EI-MS (70 eV): $m / z(\%): 648(100)[\mathrm{M}]^{+}$. Anal. calcd for $\mathrm{C}_{36} \mathrm{H}_{52} \mathrm{~N}_{2} \mathrm{OSn}$ (648.31): C 66.78, H 8.09, N 4.33; Found: C 65.19, H 8.00, N 4.13.

11.4.26. Synthesis of $\mathrm{LSnC}\left(\mathrm{CO}_{2} \mathrm{Me}\right) \mathrm{CH}_{2}$ (27)

A solution of $\mathrm{HC} \equiv \mathrm{CCO}_{2} \mathrm{Me}(0.085 \mathrm{~g}, 1.00 \mathrm{mmol}$ in $5 \mathrm{~mL}$ toluene $)$ was added drop by drop by cannula to a solution of $20(0.540 \mathrm{~g}, 1.00 \mathrm{mmol}$ in toluene $15 \mathrm{~mL})$ at room temperature. After $0.5 \mathrm{~h}$ under constant stirring at ambient temperature all volatiles were removed in vacuo, and the remaining residue was extracted with $n$ hexane $(15 \mathrm{~mL})$ and concentrated to about $5 \mathrm{~mL}$ and stored in a $-30{ }^{\circ} \mathrm{C}$ freezer. Yellow crystals of 27 suitable for X-ray diffraction analysis are formed after two days. Yield: $0.435 \mathrm{~g}(70 \%)$. Mp $170{ }^{\circ} \mathrm{C} .{ }^{1} \mathrm{H}$ NMR ( 500 $\left.\mathrm{MHz}, \mathrm{C}_{6} \mathrm{D}_{6}\right): \delta 7.08-7.14(\mathrm{~m}, 6 \mathrm{H}, \mathrm{Ar}-\mathrm{H}), 6.45\left(\mathrm{br}, 1 \mathrm{H}, \mathrm{C}=\mathrm{CH}_{2}\right), 6.15\left(\mathrm{br}, 1 \mathrm{H}, \mathrm{C}=\mathrm{CH}_{2}\right), 4.81(\mathrm{~s}, 1 \mathrm{H}, \gamma-\mathrm{CH}), 3.67$ (sept, 2H, $\left.\mathrm{CH}\left(\mathrm{CH}_{3}\right)_{2}\right), 3.42\left(\mathrm{~s}, 3 \mathrm{H}, \mathrm{CH}_{3}\right), 3.40$ (sept, 2H, $\left.\mathrm{CH}\left(\mathrm{CH}_{3}\right)_{2}\right), 1.60\left(\mathrm{~s}, 6 \mathrm{H}, \mathrm{CH}_{3}\right), 1.27(\mathrm{~m}, 12 \mathrm{H}$, $\left.\mathrm{CH}\left(\mathrm{CH}_{3}\right)_{2}\right), 1.16\left(\mathrm{~m}, 12 \mathrm{H}, \mathrm{CH}\left(\mathrm{CH}_{3}\right)_{2}\right) \mathrm{ppm} .{ }^{119} \mathrm{Sn}\left\{{ }^{1} \mathrm{H}\right\} \mathrm{NMR}(186.46 \mathrm{MHz}): \delta-93.28 \mathrm{ppm}$. EI-MS: $m / z(\%) 622$ (100) $[\mathrm{M}]^{+}$. Anal. calcd for $\mathrm{C}_{33} \mathrm{H}_{46} \mathrm{~N}_{2} \mathrm{O}_{2} \mathrm{Sn}$ (622.26): C 63.78, H 7.46, N 4.51; Found: C 63.80, H 7.84, N 4.47. 
11.4.27. Synthesis of $\mathrm{LSnC}\left(\mathrm{CO}_{2} \mathrm{Et}\right) \mathrm{CH}_{2}$ (28)

A solution of $\mathrm{HC} \equiv \mathrm{CCO}_{2} \mathrm{Et}(0.100 \mathrm{~g}, 1.00 \mathrm{mmol}$ in $5 \mathrm{~mL}$ toluene $)$ was added drop by drop by cannula to a solution of $20(0.490 \mathrm{~g}, 1.00 \mathrm{mmol}$ in toluene $15 \mathrm{~mL})$ at room temperature. After $0.5 \mathrm{~h}$ under constant stirring at ambient temperature the yellow solution remains unchanged. All volatiles were removed in vacuo, and the remaining residue was extracted with $n$-hexane $(15 \mathrm{~mL})$ and concentrated to about $5 \mathrm{~mL}$ and stored in a $-30{ }^{\circ} \mathrm{C}$ freezer. Yellow crystals of 28 are formed after one week. Yield: $0.460 \mathrm{~g}(72 \%)$. Mp $168{ }^{\circ} \mathrm{C}$. ${ }^{1} \mathrm{H}$ NMR ( 500 $\left.\mathrm{MHz}, \mathrm{C}_{6} \mathrm{D}_{6}\right): \delta 7.10-7.20(\mathrm{~m}, 6 \mathrm{H}, \mathrm{Ar}-\mathrm{H}), 6.70\left(\mathrm{br}, 1 \mathrm{H}, \mathrm{C}=\mathrm{CH}_{2}\right), 6.10\left(\mathrm{br}, 1 \mathrm{H}, \mathrm{C}=\mathrm{CH}_{2}\right), 4.82(\mathrm{~s}, 1 \mathrm{H}, \gamma-\mathrm{CH}), 4.08$ (q, 2H, CH $\mathrm{CH}_{2}, 3.67$ (sept, 2H, $\left.\mathrm{CH}\left(\mathrm{CH}_{3}\right)_{2}\right), 3.42$ (sept, 2H, $\left.\mathrm{CH}\left(\mathrm{CH}_{3}\right)_{2}\right), 1.62(\mathrm{~s}, 6 \mathrm{H}, \mathrm{CH}), 1.28(\mathrm{~m}, 12 \mathrm{H}$, $\left.\mathrm{CH}\left(\mathrm{CH}_{3}\right)_{2}\right), 1.18\left(\mathrm{~m}, 12 \mathrm{H}, \mathrm{CH}\left(\mathrm{CH}_{3}\right)_{2}\right), 1.03\left(\mathrm{t}, 3 \mathrm{H}, \mathrm{CH}_{2} \mathrm{CH}_{3}\right)$ ppm. ${ }^{119} \mathrm{Sn}\left\{{ }^{1} \mathrm{H}\right\} \mathrm{NMR}(186.46 \mathrm{MHz}): \delta-89.02$ ppm. EI-MS: $m / z(\%) 636$ (100) [M] $]^{+}$. Anal. calcd for $\mathrm{C}_{34} \mathrm{H}_{48} \mathrm{~N}_{2} \mathrm{O}_{2} \mathrm{Sn}$ (636.27): C 64.26, H 7.61, N 4.41; Found: C 63.98, H 7.92, N 4.38.

\subsubsection{Synthesis of $\mathrm{LSnC}\left(\mathrm{CO}_{2} \mathrm{Et}\right) \mathrm{CHMe}(29)$}

A solution of ethyl but-2-ynoate $(0.110 \mathrm{~g}, 1.00 \mathrm{mmol}$ in $5 \mathrm{~mL}$ toluene $)$ was added drop by drop by cannula to a solution of $20(0.54 \mathrm{~g}, 1.00 \mathrm{mmol}$ in toluene $15 \mathrm{~mL})$ at room temperature. After $12 \mathrm{~h}$ all volatiles were removed in vacuo, and the remaining residue was extracted with $n$-hexane $(15 \mathrm{~mL})$ and concentrated to about $5 \mathrm{~mL}$ and stored in a $-30{ }^{\circ} \mathrm{C}$ freezer. Yellow crystals of 29 suitable for X-ray diffraction analysis are formed after four days. Yield: $0.570 \mathrm{~g}\left(88 \%\right.$ ). Mp $129{ }^{\circ} \mathrm{C} .{ }^{1} \mathrm{H}$ NMR (500 MHz, $\left.\mathrm{C}_{6} \mathrm{D}_{6}\right): \delta 7.07-7.20(\mathrm{~m}, 6 \mathrm{H}, \mathrm{Ar}-H), 5.84(\mathrm{br}, 1 \mathrm{H}$,

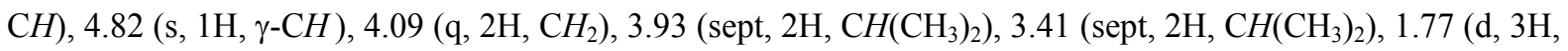
$\left.\mathrm{CH}_{3}\right), 1.64\left(\mathrm{~s}, 6 \mathrm{H}, \mathrm{CH}_{3}\right), 1.37\left(\mathrm{~d}, 6 \mathrm{H}, \mathrm{CH}\left(\mathrm{CH}_{3}\right)_{2}\right), 1.29$ (d, 6H, $\left.\mathrm{CH}\left(\mathrm{CH}_{3}\right)_{2}\right), 1.22\left(\mathrm{~d}, 6 \mathrm{H}, \mathrm{CH}\left(\mathrm{CH}_{3}\right)_{2}\right), 1.17(\mathrm{~d}, 6 \mathrm{H}$, $\left.\mathrm{CH}\left(\mathrm{CH}_{3}\right)_{2}\right), 1.11\left(\mathrm{t}, 3 \mathrm{H}, \mathrm{CH}_{3}\right) \mathrm{ppm} .{ }^{119} \mathrm{Sn}\left\{{ }^{1} \mathrm{H}\right\}$ NMR (186.46 MHz): $\delta-90 \mathrm{ppm}$. IR (Nujol, $\left.\mathrm{KBr}\right): \tilde{v}=1686 \mathrm{~cm}$ ${ }^{-1}(\mathrm{C}=\mathrm{O})$. EI-MS (70 eV): $m / z(\%): 650(100)[\mathrm{M}]^{+}$. Anal. calcd for $\mathrm{C}_{35} \mathrm{H}_{50} \mathrm{~N}_{2} \mathrm{O}_{2} \mathrm{Sn}(650.29): \mathrm{C} 67.83, \mathrm{H} \mathrm{8.67,} \mathrm{N}$ 7.53; found: C 67.78, H 8.97, N 7.69.

\subsubsection{Synthesis of $\mathrm{LSnC}\left(\mathrm{CO}_{2} \mathrm{Me}\right) \mathrm{CHCO}_{2} \mathrm{Me}(30)$}

A solution of dimethyl acetylenedicarboxylate $(0.140 \mathrm{~g}, 1.00 \mathrm{mmol}$ in $5 \mathrm{~mL}$ toluene) was added by cannula to a solution of $20(0.490 \mathrm{~g}, 1.00 \mathrm{mmol}$ in toluene $15 \mathrm{~mL})$ at room temperature. After $3 \mathrm{~h}$ all volatiles were removed in vacuo, and the remaining residue was extracted with $n$-hexane $(15 \mathrm{~mL})$. The solvent was removed in vacuo and compound 30 obtained as a red yellow solid. Yield: $0.610 \mathrm{~g}(90 \%)$. Mp $179{ }^{\circ} \mathrm{C} .{ }^{1} \mathrm{H} \mathrm{NMR}(500 \mathrm{MHz}$, $\left.\mathrm{C}_{6} \mathrm{D}_{6}\right): \delta 6.34-7.15(\mathrm{~m}, 15 \mathrm{H}, \mathrm{Ar}-H), 6.92(\mathrm{~s}, 1 \mathrm{H}, \mathrm{CH}), 6.26(\mathrm{~s}, 1 \mathrm{H}, \mathrm{CH}), 4.85(\mathrm{~s}, 1 \mathrm{H}, \gamma-\mathrm{CH}), 4.83(\mathrm{~s}, 1 \mathrm{H}, \gamma-\mathrm{CH})$, 3.88 (sept, 2H, $\left.\mathrm{CH}\left(\mathrm{CH}_{3}\right)_{2}\right), 3.66$ (s, 3H, CH $), 3.35$ (s, 3H, CH $), 3.29$ (sept, 2H, $\left.\mathrm{CH}\left(\mathrm{CH}_{3}\right)_{2}\right), 1.65(\mathrm{~s}, 6 \mathrm{H}, \mathrm{CH})$, $1.61\left(\mathrm{~s}, 6 \mathrm{H}, \mathrm{CH}_{3}\right), 1.36\left(\mathrm{~d}, 6 \mathrm{H}, \mathrm{CH}\left(\mathrm{CH}_{3}\right)_{2}\right), 1.34\left(\mathrm{~d}, 6 \mathrm{H}, \mathrm{CH}\left(\mathrm{CH}_{3}\right)_{2}\right), 1.23\left(\mathrm{~d}, 6 \mathrm{H}, \mathrm{CH}\left(\mathrm{CH}_{3}\right)_{2}\right), 1.21(\mathrm{~d}, 6 \mathrm{H}$, $\left.\mathrm{CH}\left(\mathrm{CH}_{3}\right)_{2}\right), 1.20\left(\mathrm{~d}, 6 \mathrm{H}, \mathrm{CH}\left(\mathrm{CH}_{3}\right)_{2}\right), 1.19$ (d, 6H, $\left.\mathrm{CH}\left(\mathrm{CH}_{3}\right)_{2}\right), 1.17$ (d, 6H, $\left.\mathrm{CH}\left(\mathrm{CH}_{3}\right)_{2}\right), 1.15\left(\mathrm{~d}, 6 \mathrm{H}, \mathrm{CH}(\mathrm{CH})_{2}\right)$ ppm. ${ }^{119} \mathrm{Sn}\left\{{ }^{1} \mathrm{H}\right\}$ NMR (186.46 MHz): $\delta-132,-211 \mathrm{ppm}$.

\subsubsection{Synthesis of $\mathrm{LSnC}\left(\mathrm{CO}_{2} \mathrm{Et}\right) \mathrm{CHCO}_{2} \mathrm{Et}(\mathbf{3 1})$}

A solution of diethyl acetylenedicarboxylate $(0.170 \mathrm{~g}, 1.00 \mathrm{mmol}$ in $5 \mathrm{~mL})$ toluene was added drop by drop by cannula to a solution of $\mathbf{2 0}(0.540 \mathrm{~g}, 1.00 \mathrm{mmol}$ in toluene $15 \mathrm{~mL})$ at room temperature. After $6 \mathrm{~h}$ under constant stirring at ambient temperature the yellow solution turned red. All volatiles were removed in vacuo, and the remaining residue was extracted with $n$-hexane $(15 \mathrm{~mL})$ and concentrated to about $5 \mathrm{~mL}$ and stored in a $-30{ }^{\circ} \mathrm{C}$ freezer. Red crystals of 31 suitable for X-ray diffraction analysis are formed after one day. Yield: $0.480 \mathrm{~g}$ (68 \%). Mp $138{ }^{\circ} \mathrm{C} .{ }^{1} \mathrm{H}$ NMR ( $\left.500 \mathrm{MHz}, \mathrm{C}_{6} \mathrm{D}_{6}\right): \delta 7.05-7.16(\mathrm{~m}, 6 \mathrm{H}, \mathrm{Ar}-H), 6.93(\mathrm{~s}, \mathrm{CH}), 6.26(\mathrm{~s}, \mathrm{CH}), 4.87(\mathrm{~s}$, $\gamma-\mathrm{CH}), 4.85(\mathrm{~s}, \gamma-\mathrm{CH}), 4.22\left(\mathrm{q}, \mathrm{CH}_{2}\right), 4.03\left(\mathrm{q}, \mathrm{CH}_{2}\right), 3.98\left(\mathrm{q}, \mathrm{CH}_{2}\right), 3.88\left(\mathrm{~m}, \mathrm{CH}\left(\mathrm{CH}_{3}\right)_{2}\right), 3.84\left(\mathrm{q}, \mathrm{CH}_{2}\right), 3.28(\mathrm{~m}$, $\left.\mathrm{CH}\left(\mathrm{CH}_{3}\right)_{2}\right), 1.65\left(\mathrm{~s}, \mathrm{CH}_{3}\right), 1.64\left(\mathrm{~s}, \mathrm{CH}_{3}\right), 1.35\left(\mathrm{t}, \mathrm{CH}_{3}\right), 1.32-1.12\left(\mathrm{~m}, \mathrm{CH}\left(\mathrm{CH}_{3}\right)_{2}\right), 1.06\left(\mathrm{t}, \mathrm{CH}_{3}\right), 0.95(\mathrm{t}, \mathrm{CH})$, $0.87\left(\mathrm{t}, \mathrm{CH}_{3}\right), 0.82$ (t, $\left.\mathrm{CH}_{3}\right)$ ppm. ${ }^{119} \mathrm{Sn}\left\{{ }^{1} \mathrm{H}\right\}$ NMR (186.46 MHz): $\delta-130.36,-211.20$ ppm. EI-MS: $m / z(\%) 708$ (100) $[\mathrm{M}]^{+}$. Anal. calcd for $\mathrm{C}_{37} \mathrm{H}_{52} \mathrm{~N}_{2} \mathrm{O}_{4} \mathrm{Sn}$ (708.29): C 62.87, H 7.41, N 3.96; Found: C 60.63, H 7.41, N 3.64.

\subsubsection{Synthesis of $\mathrm{LSnC}\left(\mathrm{CO}_{2} t \mathrm{Bu}\right) \mathrm{CHCO}_{2} t \mathrm{Bu}(32)$}

A solution of ditertiary butylacetylenedicarboxylate $(0.225 \mathrm{~g}, 1.00 \mathrm{mmol}$ in $5 \mathrm{~mL}$ toluene $)$ was added drop by drop by cannula to a solution of $20(0.540 \mathrm{~g}, 1.00 \mathrm{mmol}$ in toluene $15 \mathrm{~mL})$ at room temperature. After overnight constant stirring at ambient temperature, all the volatiles were removed in vacuo and the remaining residue was 
extracted with $n$-hexane $(25 \mathrm{~mL})$. Complete evaporation of the filtrate resulted in a red power of the title compound 32. Yield: $0.490 \mathrm{~g}(64 \%)$. Mp $179^{\circ} \mathrm{C}$. ${ }^{1} \mathrm{H}$ NMR ( $\left.500 \mathrm{MHz}, \mathrm{C}_{6} \mathrm{D}_{6}\right): \delta 7.06-7.15$ (m, 6H, Ar-H ), 6.93 (s, $\mathrm{CH}), 6.18$ (s, $\mathrm{CH}), 4.92$ (s, $\gamma-\mathrm{CH}), 4.90(\mathrm{~s}, \gamma-\mathrm{CH}), 3.82\left(\mathrm{~m}, 2 \mathrm{H}, \mathrm{CH}\left(\mathrm{CH}_{3}\right)_{2}\right), 3.36$ (sept, $\left.2 \mathrm{H}, \mathrm{CH}\left(\mathrm{CH}_{3}\right)_{2}\right), 1.72$ (s, $\left.6 \mathrm{H}, \mathrm{CH}_{3}\right), 1.65\left(\mathrm{~s}, 9 \mathrm{H}, \mathrm{C}\left(\mathrm{CH}_{3}\right)_{3}\right), 1.37-1.15\left(\mathrm{~m}, 24 \mathrm{H}, \mathrm{CH}\left(\mathrm{CH}_{3}\right)_{2}\right), 1.25\left(\mathrm{~s}, 9 \mathrm{H}, \mathrm{C}\left(\mathrm{CH}_{3}\right)_{3}\right) \mathrm{ppm} .{ }^{119} \mathrm{Sn}\left\{{ }^{1} \mathrm{H}\right\}$ NMR (186.46 MHz): $\delta$-123.27, $-205.75 \mathrm{ppm}$. EI-MS: $m / z(\%) 707$ (100) $[\mathrm{M}-t \mathrm{Bu}]^{+}$. Anal. calcd for $\mathrm{C}_{41} \mathrm{H}_{60} \mathrm{~N}_{2} \mathrm{O}_{4} \mathrm{Sn}$ (764.36): C 64.49, H 7.92, N 3.67; Found: C 64.38, H 8.08, N 3.59.

\subsubsection{Synthesis of 33}

A solution of $i \operatorname{PrNCN} i \operatorname{Pr}(0.210 \mathrm{~g}, 1.00 \mathrm{mmol}$ in $5 \mathrm{~mL}$ toluene) was added by cannula to a solution of 20 $(0.540 \mathrm{~g}, 1.00 \mathrm{mmol}$ in toluene $15 \mathrm{~mL})$ at room temperature. After $12 \mathrm{~h}$ all volatiles were removed in vacuo, and the remaining residue was extracted with $n$-hexane $(25 \mathrm{~mL})$. The solvent was removed and compound 33 obtained as a yellow powder. Yield: $0.610 \mathrm{~g}(82 \%)$. Mp $190{ }^{\circ} \mathrm{C} .{ }^{1} \mathrm{H}$ NMR $\left(500 \mathrm{MHz}, \mathrm{C}_{6} \mathrm{D}_{6}\right): \delta 8.26(\mathrm{~s}, 1 \mathrm{H}$, $\mathrm{CH}$ ), 7.07-7.16 (m, 6H, Ar- $H$ ), 4.80 (s, $1 \mathrm{H}, \gamma-\mathrm{CH}), 3.42$ (sept, $\left.2 \mathrm{H}, \mathrm{CH}\left(\mathrm{CH}_{3}\right)_{2}\right), 3.28$ (sept, $\left.2 \mathrm{H}, \mathrm{CH}\left(\mathrm{CH}_{3}\right)_{2}\right)$, the two other septet of the carbodiimde rings are overlapped with the base line, $1.57\left(\mathrm{~s}, 6 \mathrm{H}, \mathrm{CH}_{3}\right), 1.38-1.10(\mathrm{~m}$, $\left.36 \mathrm{H}, \mathrm{CH}\left(\mathrm{CH}_{3}\right)_{2}\right)$ ppm. ${ }^{119} \mathrm{Sn}\left\{{ }^{1} \mathrm{H}\right\}$ NMR $(186.46 \mathrm{MHz}): \delta$-177.69 ppm. EI-MS (70 eV): $\mathrm{m} / \mathrm{z}(\%): 664$ (100) [M]. Anal. calcd for $\mathrm{C}_{36} \mathrm{H}_{56} \mathrm{~N}_{4} \mathrm{Sn}$ (664.35): C 65.16, H 8.51, N 8.44; found: C 65.21, H 8.57, N 8.24.

\subsubsection{Synthesis of $\mathbf{3 4}$}

A solution of DCC $(0.210 \mathrm{~g}, 1.00 \mathrm{mmol}$ in $5 \mathrm{~mL}$ toluene $)$ was added by cannula to a solution of $20(0.540 \mathrm{~g}$, $1.00 \mathrm{mmol}$ in toluene $15 \mathrm{~mL}$ ) at room temperature. After $12 \mathrm{~h}$ all volatiles were removed in vacuo, and the remaining residue was extracted with $n$-hexane $(25 \mathrm{~mL})$. The solvent was removed and compound $\mathbf{3 4}$ obtained as a yellow powder. Yield: $0.610 \mathrm{~g}(82 \%)$. Mp $129{ }^{\circ} \mathrm{C} .{ }^{1} \mathrm{H}$ NMR $\left(500 \mathrm{MHz}, \mathrm{C}_{6} \mathrm{D}_{6}\right): \delta 8.30(\mathrm{~s}, 1 \mathrm{H}, \mathrm{CH}), 7.08-$ 7.19 (m, 6H, Ar- $H$ ), 4.80 (s, $1 \mathrm{H}, \gamma-\mathrm{CH}$ ), 3.49 (m, 2H, Cy), 3.31 (sept, 4H, $\left.\mathrm{CH}\left(\mathrm{CH}_{3}\right)_{2}\right), 1.10-1-90$ (m, 20H, Cy), 1.58 (s, 6H, $\left.\mathrm{CH}_{3}\right), 1.45$ (d, 6H, $\left.\mathrm{CH}\left(\mathrm{CH}_{3}\right)_{2}\right), 1.33\left(\mathrm{~d}, 6 \mathrm{H}, \mathrm{CH}\left(\mathrm{CH}_{3}\right)_{2}\right), 1.27$ (d, 6H, $\left.\mathrm{CH}\left(\mathrm{CH}_{3}\right)_{2}\right), 1.13$ (d, 6H, $\left.\mathrm{CH}\left(\mathrm{CH}_{3}\right)_{2}\right), 1.16\left(\mathrm{~d}, 3 \mathrm{H}, \mathrm{CH}\left(\mathrm{CH}_{3}\right)_{2}\right), 1.11$ (d, 3H, $\left.\mathrm{CH}\left(\mathrm{CH}_{3}\right)_{2}\right), 1.05$ (d, 3H, $\left.\mathrm{CH}\left(\mathrm{CH}_{3}\right)_{2}\right), 0.88$ (d, 3H, $\left.\mathrm{CH}\left(\mathrm{CH}_{3}\right)_{2}\right)$ ppm. ${ }^{119} \mathrm{Sn}\left\{{ }^{1} \mathrm{H}\right\}$ NMR (186.46 MHz): $\delta-175 \mathrm{ppm}$. EI-MS (70 eV): $\mathrm{m} / z(\%): 744$ (100) [M] ${ }^{+}$. Anal. calcd for $\mathrm{C}_{42} \mathrm{H}_{64} \mathrm{~N}_{4} \mathrm{Sn}$ (744.42): C 67.83, H 8.67, N 7.53; found: C 67.78, H 8.97, N 7.69.

\subsubsection{Synthesis of $\mathrm{LSnOCHPhCF}_{3}(\mathbf{3 5})$}

A solution of 2,2,2-trifluoro acetophenone $(0.175 \mathrm{~g}, 1.00 \mathrm{mmol}$ in $5 \mathrm{~mL}$ toluene) was added by cannula to a solution of $20(0.54 \mathrm{~g}, 1.00 \mathrm{mmol}$ in toluene $20 \mathrm{~mL})$ at room temperature. After $12 \mathrm{~h}$ all volatiles were removed in vacuo, and the remaining residue was extracted with $n$-hexane $(25 \mathrm{~mL})$. The solvent was removed and compound 35 obtained as a powder. Yield: $0.640 \mathrm{~g}(90 \%)$. Mp $172{ }^{\circ} \mathrm{C} .{ }^{1} \mathrm{H}$ NMR $\left(500 \mathrm{MHz}, \mathrm{C}_{6} \mathrm{D}_{6}\right): \delta 7.00-7.27$ $(\mathrm{m}, 11 \mathrm{H}, \mathrm{Ar}-\mathrm{H}), 5.15$ (q, $1 \mathrm{H}, \mathrm{CH}), 4.84$ (s, $1 \mathrm{H}, \gamma-\mathrm{CH}), 3.76$ (sept, $\left.1 \mathrm{H}, \mathrm{CH}\left(\mathrm{CH}_{3}\right)_{2}\right), 3.63$ (sept, $\left.1 \mathrm{H}, \mathrm{CH}\left(\mathrm{CH}_{3}\right)_{2}\right)$, 3.10 (sept, $\left.1 \mathrm{H}, \mathrm{CH}\left(\mathrm{CH}_{3}\right)_{2}\right), 3.04$ (sept, $\left.1 \mathrm{H}, \mathrm{CH}\left(\mathrm{CH}_{3}\right)_{2}\right), 1.56$ (s, $\left.3 \mathrm{H}, \mathrm{CH}_{3}\right), 1.50$ (s, $\left.3 \mathrm{H}, \mathrm{CH}_{3}\right), 1.43$ (d, $3 \mathrm{H}$, $\left.\mathrm{CH}\left(\mathrm{CH}_{3}\right)_{2}\right), 1.36\left(\mathrm{~d}, 3 \mathrm{H}, \mathrm{CH}\left(\mathrm{CH}_{3}\right)_{2}\right), 1.20\left(\mathrm{~d}, 3 \mathrm{H}, \mathrm{CH}\left(\mathrm{CH}_{3}\right)_{2}\right), 1.18\left(\mathrm{~d}, 3 \mathrm{H}, \mathrm{CH}\left(\mathrm{CH}_{3}\right)_{2}\right), 1.12\left(\mathrm{~d}, 3 \mathrm{H}, \mathrm{CH}\left(\mathrm{CH}_{3}\right)_{2}\right)$, 1.09 (d, 3H, $\left.\mathrm{CH}\left(\mathrm{CH}_{3}\right)_{2}\right), 1.07$ (d, 3H, $\left.\mathrm{CH}\left(\mathrm{CH}_{3}\right)_{2}\right), 1.00\left(\mathrm{~d}, 3 \mathrm{H}, \mathrm{CH}\left(\mathrm{CH}_{3}\right)_{2}\right) \mathrm{ppm} .{ }^{19} \mathrm{~F}\left\{{ }^{1} \mathrm{H}\right\} \operatorname{NMR}(188.29 \mathrm{MHz}): \delta$ -76.29 (d, 3F, $\left.\mathrm{CF}_{3}\right) \mathrm{ppm} .{ }^{119} \mathrm{Sn}\left\{{ }^{1} \mathrm{H}\right\}$ NMR (186.46 MHz): $\delta$-252 ppm. EI-MS (70 eV): $\mathrm{m} / z(\%): 712(100)[\mathrm{M}]^{+}$. Anal. calcd for $\mathrm{C}_{37} \mathrm{H}_{47} \mathrm{~F}_{3} \mathrm{~N}_{2} \mathrm{OSn}$ (712.27): C 62.46, H 6.66, N 3.94; Found: C 62.50, H 6.77, N 3.98.

\subsubsection{Synthesis of $\mathrm{LSnOCH}\left(2-\mathrm{C}_{4} \mathrm{H}_{3} \mathrm{~S}\right) \mathrm{CF}_{3}$ (36)}

A solution of 2,2,2-trifluoro2-thiophene $(0.180 \mathrm{~g}, 1.00 \mathrm{mmol}$ in $5 \mathrm{~mL}$ toluene) was added by cannula to a solution of $20(0.54 \mathrm{~g}, 1.00 \mathrm{mmol}$ in toluene $20 \mathrm{~mL})$ at room temperature. After $12 \mathrm{~h}$ all volatiles were removed in vacuo, and the remaining residue was extracted with $n$-hexane $(25 \mathrm{~mL})$. The resulting solution was concentrated and stored in a freezer, after three days yellow crystals of $\mathbf{3 6}$ are formed which are suitable for Xray structural analysis. Yield: $0.610 \mathrm{~g}(85 \%)$. Mp $158^{\circ} \mathrm{C} .{ }^{1} \mathrm{H}$ NMR $\left(500 \mathrm{MHz}, \mathrm{C}_{6} \mathrm{D}_{6}\right): \delta 7.03-7.20(\mathrm{~m}, 6 \mathrm{H}, \mathrm{Ar}-H$ ), $6.81\left(\mathrm{~m}, 2 \mathrm{H}, \mathrm{C}_{4} H_{3} \mathrm{~S}\right), 6.64\left(\mathrm{~m}, 1 \mathrm{H}, \mathrm{C}_{4} H_{3} \mathrm{~S}\right), 5.45$ (q, $\left.1 \mathrm{H}, \mathrm{CH}\right), 4.87$ (s, $\left.1 \mathrm{H}, \gamma-\mathrm{CH}\right), 3.78$ (sept, $\left.1 \mathrm{H}, \mathrm{CH}\left(\mathrm{CH}_{3}\right)_{2}\right)$, 3.61 (sept, 1H, $\left.\mathrm{CH}\left(\mathrm{CH}_{3}\right)_{2}\right), 3.30$ (sept, $\left.1 \mathrm{H}, \mathrm{CH}\left(\mathrm{CH}_{3}\right)_{2}\right), 3.09$ (sept, $\left.1 \mathrm{H}, \mathrm{CH}\left(\mathrm{CH}_{3}\right)_{2}\right), 1.57$ (s, 3H, $\left.\mathrm{CH}_{3}\right), 1.53$ (s, $\left.3 \mathrm{H}, \mathrm{CH}_{3}\right), 1.42$ (d, 3H, $\left.\mathrm{CH}\left(\mathrm{CH}_{3}\right)_{2}\right), 1.37$ (d, 3H, $\left.\mathrm{CH}\left(\mathrm{CH}_{3}\right)_{2}\right), 1.24$ (d, 3H, $\left.\mathrm{CH}\left(\mathrm{CH}_{3}\right)_{2}\right), 1.21$ (d, 3H, $\left.\mathrm{CH}\left(\mathrm{CH}_{3}\right)_{2}\right)$, 1.19-1.12 (m, 12H, CH(CH $\left(\mathrm{CH}_{3}\right)$ ) ppm. ${ }^{19} \mathrm{~F}\left\{{ }^{1} \mathrm{H}\right\}$ NMR (188.29 MHz): $\delta-77.39$ (br, $\left.3 \mathrm{~F}, \mathrm{C} F_{3}\right) \mathrm{ppm} .{ }^{119} \mathrm{Sn}\left\{{ }^{1} \mathrm{H}\right\} \mathrm{NMR}$ (186.46 MHz): $\delta$-262 ppm. EI-MS (70 eV): $m / z(\%): 718(100)[\mathrm{M}]^{+}$. Anal. calcd for $\mathrm{C}_{35} \mathrm{H}_{45} \mathrm{~F}_{3} \mathrm{~N}_{2} \mathrm{OSSn}(718.22)$ : C 58.59, H 6.32, N 3.90, S 4.47; Found: C 58.57, H 6.52, N 3.84, S 4.81. 
11.4.36. Synthesis of $\mathrm{LSnOCHPh}\left(\mathrm{C}_{6} \mathrm{~F}_{5}\right)(37)$

In a NMR tube $\mathrm{PhCOC}_{6} \mathrm{~F}_{5}(0.136 \mathrm{~g}, 0.50 \mathrm{mmol})$ and $20(0.270 \mathrm{~g}, 0.50 \mathrm{mmol})$ were talken and $1 \mathrm{~mL}_{6} \mathrm{C}_{6}$ was added at room temperature. After $24 \mathrm{~h}$ measuring the NMR showing the formation of title compound 37 along with the $\mathrm{LSnF}$ and $\mathrm{PhCO}\left(4-\mathrm{C}_{6} \mathrm{~F}_{4} \mathrm{H}\right) .{ }^{1} \mathrm{H}$ NMR $\left(200 \mathrm{MHz}, \mathrm{C}_{6} \mathrm{D}_{6}\right): \delta 6.85-7.65(\mathrm{~m}, 6 \mathrm{H}, \mathrm{Ar}-H), 6.41(\mathrm{~s}, 1 \mathrm{H}, \mathrm{CH})$, 4.78 (s, 1H, $\gamma$-CH), 3.56 (sept, 2H, $\left.\mathrm{CH}\left(\mathrm{CH}_{3}\right)_{2}\right), 3.13$ (sept, 2H, $\left.\mathrm{CH}\left(\mathrm{CH}_{3}\right)_{2}\right), 1.53$ (s, 6H, $\left.\mathrm{CH}_{3}\right), 1.44$ (d, 6H, $\left.\mathrm{CH}\left(\mathrm{CH}_{3}\right)_{2}\right), 1.23\left(\mathrm{~d}, 6 \mathrm{H}, \mathrm{CH}\left(\mathrm{CH}_{3}\right)_{2}\right), 1.11\left(\mathrm{~d}, 6 \mathrm{H}, \mathrm{CH}\left(\mathrm{CH}_{3}\right)_{2}\right), 0.90\left(\mathrm{~d}, 6 \mathrm{H}, \mathrm{CH}\left(\mathrm{CH}_{3}\right)_{2}\right) .{ }^{119} \mathrm{Sn}\left\{{ }^{1} \mathrm{H}\right\} \mathrm{NMR}(186.46$ $\mathrm{MHz}): \delta-217 \mathrm{ppm}$.

11.4.37. Synthesis of $\mathrm{LSnOCH}\left(\mathrm{C}_{6} \mathrm{~F}_{5}\right)_{2}$ (38)

A solution of $\left.\mathrm{C}_{6} \mathrm{~F}_{5} \mathrm{COC}_{6} \mathrm{~F}_{5}\right)(0.360 \mathrm{~g}, 1.00 \mathrm{mmol}$ in $10 \mathrm{~mL}$ toluene) was added by cannula to a solution of $\mathbf{2 0}$ $(0.54 \mathrm{~g}, 1.00 \mathrm{mmol}$ in toluene $20 \mathrm{~mL})$ at room temperature. After $24 \mathrm{~h}$ all volatiles were removed in vacuo, and the remaining residue was extracted with $n$-hexane $(25 \mathrm{~mL})$. After evaporation of solvent the title compounds 38 are obtained along with the $\mathrm{LSnF}$ and $\mathrm{C}_{6} \mathrm{~F}_{5} \mathrm{CO}\left(4-\mathrm{C}_{6} \mathrm{~F}_{4} \mathrm{H}\right) .{ }^{1} \mathrm{H}$ NMR $\left(500 \mathrm{MHz}, \mathrm{C}_{6} \mathrm{D}_{6}\right): \delta 6.99-7.13(\mathrm{~m}, 15 \mathrm{H}$, Ar$H$ ), $6.72\left(\mathrm{~s},{ }^{1} \mathrm{H}, \mathrm{CH}\right), 4.77$ (s, $\left.1 \mathrm{H}, \gamma-\mathrm{CH}\right), 3.46$ (sept, $\left.2 \mathrm{H}, \mathrm{CH}\left(\mathrm{CH}_{3}\right)_{2}\right), 3.02$ (sept, $\left.2 \mathrm{H}, \mathrm{CH}\left(\mathrm{CH}_{3}\right)_{2}\right), 1.48(\mathrm{~s}, 6 \mathrm{H}$, $\left.\mathrm{CH}_{3}\right), 1.17$ (d, 6H, $\left.\mathrm{CH}\left(\mathrm{CH}_{3}\right)_{2}\right), 1.14$ (d, 6H, CH(CH$\left.)_{2}\right), 1.08$ (d, 6H, $\left.\mathrm{CH}\left(\mathrm{CH}_{3}\right)_{2}\right), 1.06$ (d, 6H, $\left.\mathrm{CH}\left(\mathrm{CH}_{3}\right)_{2}\right)$. ${ }^{119} \mathrm{Sn}\left\{{ }^{1} \mathrm{H}\right\}$ NMR (186.46 MHz): $\delta$-252 ppm. EI-MS (70 eV): $m / z(\%): 900$ (100) [M] $]^{+}$

\subsubsection{Synthesis of $\mathrm{LSnOCHPh}\left(4-\mathrm{C}_{6} \mathrm{H}_{4} \mathrm{~F}\right)(\mathbf{3 9})$}

A solution of $\mathrm{PhCO}\left(4-\mathrm{C}_{6} \mathrm{H}_{4} \mathrm{~F}\right)(0.200 \mathrm{~g}, 1.00 \mathrm{mmol}$ in $10 \mathrm{~mL}$ toluene $)$ was added by cannula to a solution of $\mathbf{2 0}$ $(0.54 \mathrm{~g}, 1.00 \mathrm{mmol}$ in toluene $20 \mathrm{~mL})$ at room temperature. After $24 \mathrm{~h}$ all volatiles were removed in vacuo, and the remaining residue was extracted with $n$-hexane $(25 \mathrm{~mL})$. After evaporation of solvent the title compounds 39 are obtained. Yield: $0.605 \mathrm{~g}(82 \%)$. Mp $130{ }^{\circ} \mathrm{C} .{ }^{1} \mathrm{H}$ NMR (300 MHz, $\left.\mathrm{C}_{6} \mathrm{D}_{6}\right): \delta 6.65-7.63(\mathrm{~m}, 15 \mathrm{H}, \mathrm{Ar}-H), 5.88$ (s, $1 \mathrm{H}, \mathrm{CH}), 4.75$ (s, $1 \mathrm{H}, \gamma-\mathrm{CH}), 3.63$ (sept, $\left.2 \mathrm{H}, \mathrm{CH}\left(\mathrm{CH}_{3}\right)_{2}\right), 3.11$ (sept, $\left.2 \mathrm{H}, \mathrm{CH}\left(\mathrm{CH}_{3}\right)_{2}\right), 1.55\left(\mathrm{~s}, 6 \mathrm{H}, \mathrm{CH}_{3}\right), 1.20$ (d, 3H, $\left.\mathrm{CH}\left(\mathrm{CH}_{3}\right)_{2}\right), 1.18$ (d, 3H, $\left.\mathrm{CH}\left(\mathrm{CH}_{3}\right)_{2}\right), 1.13$ (d, 3H, $\left.\mathrm{CH}\left(\mathrm{CH}_{3}\right)_{2}\right), 1.12$ (d, 3H, $\left.\mathrm{CH}\left(\mathrm{CH}_{3}\right)_{2}\right), 1.11$ (d, 3H, $\left.\mathrm{CH}\left(\mathrm{CH}_{3}\right)_{2}\right), 1.10$ (d, 3H, $\left.\mathrm{CH}\left(\mathrm{CH}_{3}\right)_{2}\right), 1.08$ (d, 3H, $\left.\mathrm{CH}\left(\mathrm{CH}_{3}\right)_{2}\right), 1.07$ (d, 3H, $\left.\mathrm{CH}\left(\mathrm{CH}_{3}\right)_{2}\right) .{ }^{19} \mathrm{~F} \mathrm{NMR}(188.29 \mathrm{MHz}$, $\left.\mathrm{C}_{6} \mathrm{D}_{6}\right): \delta-112.3$ (m, 1F, $p$-F). ${ }^{119} \mathrm{Sn}\left\{{ }^{1} \mathrm{H}\right\}$ NMR (186.46 MHz): $\delta-212$ ppm. EI-MS (70 eV): $m / z(\%): 737(90)$ $[\mathrm{M}]^{+}, 652$ (100) $\left[\mathrm{M}-\mathrm{C}_{6} \mathrm{H}_{4} \mathrm{~F}\right]^{+}$. Anal. calcd for $\mathrm{C}_{42} \mathrm{H}_{51} \mathrm{FN}_{2} \mathrm{OSn}$ (738.30): C 68.39, H 6.97, N 3.80; Found: C 68.75, H 7.16, N 3.62 .

\subsubsection{Synthesis of $\mathrm{LSnOCH}_{2} \mathrm{C}_{6} \mathrm{~F}_{5}$ (40)}

A solution of $\mathrm{C}_{6} \mathrm{~F}_{5} \mathrm{CHO}(0.196 \mathrm{~g}, 1.00 \mathrm{mmol}$ in $5 \mathrm{~mL}$ toluene) was added by cannula to a solution of $20(0.54 \mathrm{~g}$, $1.00 \mathrm{mmol}$ in toluene $20 \mathrm{~mL}$ ) at room temperature. After $24 \mathrm{~h}$ all volatiles were removed in vacuo, and the remaining residue was extracted with $n$-hexane $(25 \mathrm{~mL})$ and concentrated to about $15 \mathrm{~mL}$ and stored in a $-30{ }^{\circ} \mathrm{C}$ freezer. After four days yellow crystals of $\mathbf{4 0}$ are formed. Yield: $0.600 \mathrm{~g}(82 \%)$. Mp $138{ }^{\circ} \mathrm{C}$. ${ }^{1} \mathrm{H}$ NMR (300 $\left.\mathrm{MHz}, \mathrm{C}_{6} \mathrm{D}_{6}\right): \delta 6.95-7.13(\mathrm{~m}, 6 \mathrm{H}, \mathrm{Ar}-\mathrm{H}), 4.80(\mathrm{~s}, 1 \mathrm{H}, \gamma-\mathrm{CH}), 4.67\left(\mathrm{t},{ }^{4} J(\mathrm{~F}-\mathrm{H})=4.18 \mathrm{~Hz}, 2 \mathrm{H}, \mathrm{CH}_{2}\right), 3.57$ (sept, $\left.2 \mathrm{H}, \mathrm{CH}\left(\mathrm{CH}_{3}\right)_{2}\right), 3.13$ (sept, $\left.2 \mathrm{H}, \mathrm{CH}\left(\mathrm{CH}_{3}\right)_{2}\right), 1.58$ (s, $\left.6 \mathrm{H}, \mathrm{CH}_{3}\right), 1.31$ (d, $\left.6 \mathrm{H}, \mathrm{CH}\left(\mathrm{CH}_{3}\right)_{2}\right), 1.24$ (d, $\left.6 \mathrm{H}, \mathrm{CH}\left(\mathrm{CH}_{3}\right)_{2}\right)$, $1.20\left(\mathrm{~d}, 6 \mathrm{H}, \mathrm{CH}\left(\mathrm{CH}_{3}\right)_{2}\right), 1.09\left(\mathrm{~d}, 6 \mathrm{H}, \mathrm{CH}\left(\mathrm{CH}_{3}\right)_{2}\right) .{ }^{19} \mathrm{~F} \mathrm{NMR}\left(188.29 \mathrm{MHz}, \mathrm{C}_{6} \mathrm{D}_{6}\right): \delta-143.9$ (dd, $\left.2 \mathrm{~F}, o-\mathrm{F}\right),-$ 158.95 (t, $2 \mathrm{~F}, p-\mathrm{F}),-163.95$ (td, 2F, $m-\mathrm{F}) .{ }^{119} \mathrm{Sn}\left\{{ }^{1} \mathrm{H}\right\}$ NMR (186.46 MHz): $\delta-262 \mathrm{ppm}$. EI-MS $(70 \mathrm{eV}): \mathrm{m} / \mathrm{z}$ (\%): 537 (100) $\left[\mathrm{M}-\mathrm{OCH}_{2} \mathrm{C}_{6} \mathrm{~F}_{5}\right]^{+}, 198(100)\left[\mathrm{OCH}_{2} \mathrm{C}_{6} \mathrm{~F}_{5}\right]^{+}$.

\subsubsection{Synthesis of $(\mathrm{CH} t \mathrm{BuN})_{2} \mathrm{CH}_{2}(\mathbf{4 1})$}

1,3-Di-tert-butylimidazol-2-ylidene $(0.360 \mathrm{~g}, 2.0 \mathrm{mmol})$ and $\mathrm{NH}_{3} \mathrm{BH}_{3}(0.060 \mathrm{~g}, 2.0 \mathrm{mmol})$ were dissolved in toluene $(30 \mathrm{~mL})$ at room temperature. The reaction mixture was stirred overnight, then the solvent was removed in vacuo and the residue was extracted with $n$-hexane $(30 \mathrm{~mL})$. Yield: $0.630 \mathrm{~g}(95 \%) .{ }^{1} \mathrm{H}$ NMR $(200 \mathrm{MHz}$, $\left.\mathrm{C}_{6} \mathrm{D}_{6}\right): \delta 5.48(\mathrm{~s}, 2 \mathrm{H}, \mathrm{C}-H), 4.24\left(\mathrm{~s}, 2 \mathrm{H}, \mathrm{CH}_{2}\right), 1.01\left(\mathrm{~s}, 18 \mathrm{H}, \mathrm{C}\left(\mathrm{CH}_{3}\right)_{3}\right) \mathrm{ppm}$.

\subsubsection{Synthesis of $\mathrm{LGeNH}_{2}$ (42)}

Dry ammonia gas was added to a red solution of $19(0.490 \mathrm{~g}, 1 \mathrm{mmol})$ in toluene $(20 \mathrm{~mL})$ at room temperature. The reaction mixture became orange. Futher 5 min the ammonia gas was bubbled through the solution. After that the solvent was removed in vacuo and the residue was extracted with $n$-hexane. The solution was reduced to half of the volume. Storage of the solution at $-30{ }^{\circ} \mathrm{C}$ for one day yielded yellow crystals of $\mathbf{4 2}$ suitable for a 
single crystal X-ray diffraction experiment.. Yield: $0.455 \mathrm{~g}(95 \%)$. m.p. $162{ }^{\circ} \mathrm{C} .{ }^{1} \mathrm{H}$ NMR $\left(500 \mathrm{MHz}, \mathrm{C}_{6} \mathrm{D}_{6}\right): \delta$ 7.12-7.18 (m, 6H, Ar- $H$ ), 4.79 (s, $1 \mathrm{H}, \gamma-\mathrm{CH}), 3.54$ (sept, $\left.2 \mathrm{H}, \mathrm{CH}\left(\mathrm{CH}_{3}\right)_{2}\right), 3.47$ (sept, $\left.2 \mathrm{H}, \mathrm{CH}\left(\mathrm{CH}_{3}\right)_{2}\right), 1.60$ (s, 6H, $\left.\mathrm{CH}_{3}\right), 1.35$ (d, 6H, $\left.\mathrm{CH}\left(\mathrm{CH}_{3}\right)_{2}\right), 1.28\left(\mathrm{~d}, 6 \mathrm{H}, \mathrm{CH}\left(\mathrm{CH}_{3}\right)_{2}\right), 1.24$ (d, 6H, $\left.\mathrm{CH}\left(\mathrm{CH}_{3}\right)_{2}\right), 1.14\left(\mathrm{~d}, 6 \mathrm{H}, \mathrm{CH}\left(\mathrm{CH}_{3}\right)_{2}\right)$ ppm. IR (Nujol, KBr): $\tilde{v}=3431 \mathrm{vw}, 3333 \mathrm{vw}(\mathrm{N}-\mathrm{H}) \mathrm{cm}^{-1}$. EI-MS (70 eV): $\mathrm{m} / z(\%): 507$ (100) [M] ${ }^{+}$. Anal. calcd for $\mathrm{C}_{29} \mathrm{H}_{43} \mathrm{GeN}_{3}$ (507.27): C 68.79, H 8.56, N 8.30; Found: C 69.25, H 8.82, N 7.66.

11.4.42. Synthesis of $\mathrm{LGeNHNH}_{2}$ (43)

$\mathrm{NH}_{2} \mathrm{NH}_{2}(1 \mathrm{~mL}, 1 \mathrm{M}$ in THF) was added to a yellow solution of $19(0.490 \mathrm{~g}, 1 \mathrm{mmol})$ in toluene $(20 \mathrm{~mL})$ at 0 ${ }^{\circ} \mathrm{C}$. Then the reaction mixture was allowed to come to room temperature. After that the reaction mixture was stirred for additional $30 \mathrm{~min}$. All the volatiles were removed in vacuo and the residue was extracted with $n$ hexane $(30 \mathrm{~mL})$. The solution was reduced to half of the volume. Storage of the solution at $-32{ }^{\circ} \mathrm{C}$ in a freezer for two days yielded $\mathbf{4 3}$ as colorless crystals suitable for single crystal X-ray diffraction studies. Yield: $0.40 \mathrm{~g}$ (85\%); Mp $134{ }^{\circ} \mathrm{C} .{ }^{1} \mathrm{H}$ NMR (300 MHz, $\left.\mathrm{C}_{6} \mathrm{D}_{6}\right): \delta 7.10-7.17(\mathrm{~m}, 6 \mathrm{H}, \mathrm{Ar}-H)$ ), $4.71(\mathrm{~s}, 1 \mathrm{H}, \gamma-\mathrm{CH}), 3.50-3.43(\mathrm{~m}$, $\left.4 \mathrm{H}, \mathrm{CH}\left(\mathrm{CH}_{3}\right)_{2}\right), 3.16\left(\mathrm{~d}, 1 \mathrm{H}, \mathrm{NH} \mathrm{H}_{2}\right), 1.59(\mathrm{~s}, 6 \mathrm{H}, \mathrm{CH}), 1.41$ (d, 6H, $\left.\mathrm{CH}\left(\mathrm{CH}_{3}\right)_{2}\right), 1.33$ (d, 6H, $\left.\mathrm{CH}\left(\mathrm{CH}_{3}\right)_{2}\right), 1.25$ (d, $\left.6 \mathrm{H}, \mathrm{CH}\left(\mathrm{CH}_{3}\right)_{2}\right), 1.13$ (d, 6H, CH(CH $\left.\mathrm{CH}_{2}\right), 0.87$ (t, 1H, NH) ppm. EI-MS (70 eV): m/z (\%): 475 (100) [M$\left.\left(\mathrm{NHNH}_{2}, \mathrm{Me}\right)\right]^{+}$. Anal. calcd for $\mathrm{C}_{29} \mathrm{H}_{44} \mathrm{GeN}_{4}$ (522.28): C 66.81, H 8.51, N 10.75; Found: C 66.73, H 8.97, N 9.70 .

11.4.43. Synthesis of $\mathrm{LGeOPh}(\mathbf{4 4})$

A $10 \mathrm{~mL}$ toluene solution of phenol $(0.095 \mathrm{~g}, 1 \mathrm{mmol})$ was added to a solution of $19(0.490 \mathrm{~g}, 1 \mathrm{mmol})$ in toluene $(20 \mathrm{~mL})$ under stirring at room temperature and the color of the solution changed immediately from red to colorless. After $30 \mathrm{~min}$ all the volatiles were removed in vacuo and the residue extracted with $n$-hexane (30 $\mathrm{mL}$ ) and concentrated to give colorless crystals of $\mathbf{4 4}$ after two days, which are suitable for X-ray structural analysis. Yield: $0.475 \mathrm{~g}(82 \%)$; $\mathrm{Mp} 203{ }^{\circ} \mathrm{C} .{ }^{1} \mathrm{H}$ NMR $\left(500 \mathrm{MHz}, \mathrm{C}_{6} \mathrm{D}_{6}\right): \delta 6.63-7.13(\mathrm{~m}, 11 \mathrm{H}, \mathrm{Ar}-H), 4.98(\mathrm{~s}$, $1 \mathrm{H}, \gamma-\mathrm{CH}$ ), 3.77 (sept, $\left.2 \mathrm{H}, \mathrm{CH}\left(\mathrm{CH}_{3}\right)_{2}\right), 3.27$ (sept, $\left.2 \mathrm{H}, \mathrm{CH}\left(\mathrm{CH}_{3}\right)_{2}\right), 1.60$ (s, $\left.6 \mathrm{H}, \mathrm{CH}_{3}\right), 1.28\left(\mathrm{~d}, 6 \mathrm{H}, \mathrm{CH}\left(\mathrm{CH}_{3}\right)_{2}\right)$, 1.18 (d, $\left.6 \mathrm{H}, \mathrm{CH}\left(\mathrm{CH}_{3}\right)_{2}\right), 1.10$ (d, 6H, $\left.\mathrm{CH}\left(\mathrm{CH}_{3}\right)_{2}\right), 1.06$ (d, 6H, $\left.\mathrm{CH}\left(\mathrm{CH}_{3}\right)_{2}\right)$ ppm. EI-MS (70 eV): $\mathrm{m} / z$ (\%): 491 (100) $[\mathrm{M}-\mathrm{OPh}]^{+}$. Anal. calcd for $\mathrm{C}_{35} \mathrm{H}_{46} \mathrm{GeN}_{2} \mathrm{O}$ (583.39): C 72.06, H 7.95, N 4.80; Found: C 70.18, H 7.91, N 4.59 .

11.4.44. Synthesis of $\mathrm{LGeOC}_{6} \mathrm{~F}_{5}$ (45)

A $10 \mathrm{~mL}$ toluene solution of $\mathrm{C}_{6} \mathrm{~F}_{5} \mathrm{OH}(0.190 \mathrm{~g}, 1 \mathrm{mmol})$ was added to a solution of $19(0.490 \mathrm{~g}, 1 \mathrm{mmol})$ in toluene $(20 \mathrm{~mL})$ under stirring at room temperature and the color of the solution changed immediately from red to colorless. After $30 \mathrm{~min}$ all the volatiles were removed in vacuo and the residue extracted with $n$-hexane (30 $\mathrm{mL}$ ) and concentrated to give colorless crystals of $\mathbf{4 5}$ after four days, which are suitable for X-ray structural analysis. Yield: $0.540 \mathrm{~g}(80 \%)$; $\mathrm{Mp} 155^{\circ} \mathrm{C} .{ }^{1} \mathrm{H}$ NMR $\left(500 \mathrm{MHz}, \mathrm{C}_{6} \mathrm{D}_{6}\right): \delta 6.99-7.10(\mathrm{~m}, 6 \mathrm{H}, \mathrm{Ar}-H), 5.10(\mathrm{~s}, 1 \mathrm{H}$, $\gamma$ - $\mathrm{CH}$ ), 3.48 (sept, 2H, $\left.\mathrm{CH}\left(\mathrm{CH}_{3}\right)_{2}\right), 3.05$ (sept, $\left.2 \mathrm{H}, \mathrm{CH}\left(\mathrm{CH}_{3}\right)_{2}\right), 1.55$ (s, $\left.6 \mathrm{H}, \mathrm{CH}_{3}\right), 1.18$ (d, $\left.6 \mathrm{H}, \mathrm{CH}\left(\mathrm{CH}_{3}\right)_{2}\right), 1.11$ (d, 6H, $\left.\mathrm{CH}\left(\mathrm{CH}_{3}\right)_{2}\right), 1.03\left(\mathrm{~d}, 6 \mathrm{H}, \mathrm{CH}\left(\mathrm{CH}_{3}\right)_{2}\right), 0.88\left(\mathrm{~d}, 6 \mathrm{H}, \mathrm{CH}\left(\mathrm{CH}_{3}\right)_{2}\right) \mathrm{ppm} .{ }^{19} \mathrm{~F}\left\{{ }^{1} \mathrm{H}\right\} \mathrm{NMR}\left(188.31 \mathrm{MHz}, \mathrm{C}_{6} \mathrm{D}_{6}\right)$ : $\delta-158.8(\mathrm{~d}, 6 \mathrm{~F}, o-F),-166.9(\mathrm{t}, 6 \mathrm{~F}, m-F),-175.2(\mathrm{t}, 3 \mathrm{~F}, p-F)$. EI-MS (70 eV): $m / z(\%): 673(100)[\mathrm{M}]^{+}$. Anal. calcd for $\mathrm{C}_{35} \mathrm{H}_{41} \mathrm{~F}_{5} \mathrm{GeN}_{2} \mathrm{O}$ (673.34): C 62.43, H 6.14, N 4.16; Found: C 62.24, H 6.05, N 4.16.

11.4.45. Synthesis of $\mathrm{LGeOC}(\mathrm{O}) \mathrm{Ph}(46)$

A $10 \mathrm{~mL}$ toluene solution of $\mathrm{PhCO}_{2} \mathrm{H}(0.122 \mathrm{~g}, 1 \mathrm{mmol})$ was added to a solution of $19(0.490 \mathrm{~g}, 1 \mathrm{mmol})$ in toluene $(20 \mathrm{~mL})$ under stirring at room temperature and the color of the solution changed immediately from red to colorless. After $30 \mathrm{~min}$ all the volatiles were removed in vacuo and the residue extracted with $n$-hexane (30 $\mathrm{mL}$ ) and concentrated to give colorless crystals of $\mathbf{4 6}$ after one day, which are suitable for X-ray structural analysis. Yield: $0.520 \mathrm{~g}(85 \%)$; $\mathrm{Mp} 167{ }^{\circ} \mathrm{C} .{ }^{1} \mathrm{H}$ NMR $\left(500 \mathrm{MHz}, \mathrm{C}_{6} \mathrm{D}_{6}\right): \delta 6.99-8.44(\mathrm{~m}, 11 \mathrm{H}, \mathrm{Ar}-H$ ), 5.16 (s, $1 \mathrm{H}, \gamma-\mathrm{CH}$ ), 3.61 (sept, $\left.2 \mathrm{H}, \mathrm{CH}\left(\mathrm{CH}_{3}\right)_{2}\right), 3.17$ (sept, $\left.2 \mathrm{H}, \mathrm{CH}\left(\mathrm{CH}_{3}\right)_{2}\right), 1.61$ (s, $\left.6 \mathrm{H}, \mathrm{CH}_{3}\right), 1.21$ (d, $\left.6 \mathrm{H}, \mathrm{CH}\left(\mathrm{CH}_{3}\right)_{2}\right)$, 1.13 (d, $\left.6 \mathrm{H}, \mathrm{CH}\left(\mathrm{CH}_{3}\right)_{2}\right), 1.07$ (d, 6H, $\left.\mathrm{CH}\left(\mathrm{CH}_{3}\right)_{2}\right), 1.05$ (d, 6H, $\left.\mathrm{CH}\left(\mathrm{CH}_{3}\right)_{2}\right)$ ppm. EI-MS (70 eV): $\mathrm{m} / z(\%): 612$ (100) $[\mathrm{M}]^{+}$. Anal. calcd for $\mathrm{C}_{36} \mathrm{H}_{46} \mathrm{GeN}_{2} \mathrm{O}_{2}$ (611.40): C 70.72, H 7.58, N 4.55; Found: C 70.11, H 7.52, N 4.55. 
11.4.46. Synthesis of $\mathrm{L}^{\prime \prime} \mathrm{GePPh}_{2}(47)$

To a cooled $(-60 \mathrm{oC})$ solution of $\mathbf{1 9}(1.40 \mathrm{~g}, 2.86 \mathrm{mmol})$ in $n$-hexane $(30 \mathrm{~mL})$ was added a solution of $\mathrm{Ph}_{2} \mathrm{PCl}$ $(0.64 \mathrm{~g}, 2.90 \mathrm{mmol})$ in $n$-hexane $(20 \mathrm{~mL})$. The solution turned yellow and a yellow precipitate started forming while the mixture was brought to rt. After stirring the mixture overnight it was filtered and the precipitate was dried under vacuum. Keeping the filtrate at room temperature for one day also afforded orange yellow crystals of 47 along with some powder (1.82 g. Yield $1.82 \mathrm{~g}(90 \%)$. Mp $174{ }^{\circ} \mathrm{C} .{ }^{1} \mathrm{H}$ NMR $\left(500 \mathrm{MHz}, \mathrm{C}_{6} \mathrm{D}_{6}\right) \delta 1.09$ (d, $\left.3 \mathrm{H}, \mathrm{CH}\left(\mathrm{CH}_{3}\right)_{2}\right), 1.15$ (d, 3H, $\left.\mathrm{CH}\left(\mathrm{CH}_{3}\right)_{2}\right), 1.26-1.32$ (3d, 9H, $\left.\mathrm{CH}\left(\mathrm{CH}_{3}\right)_{2}\right), 1.39$ (s, 3H, $\left.\mathrm{CH}_{3}\right), 1.42$ (d, 3H, $\left.\mathrm{CH}\left(\mathrm{CH}_{3}\right)_{2}\right), 1.47$ (d, 3H, $\left.\mathrm{CH}\left(\mathrm{CH}_{3}\right)_{2}\right), 1.52\left(\mathrm{~d}, 3 \mathrm{H}, \mathrm{CH}\left(\mathrm{CH}_{3}\right)_{2}\right), 3.14$ (q, $\left.2 \mathrm{H}, \mathrm{CH}_{2}\right), 3.23\left(\mathrm{~m}, 1 \mathrm{H}, \mathrm{CH}\left(\mathrm{CH}_{3}\right)_{2}\right), 3.39$ $\left(\mathrm{m}, 1 \mathrm{H}, \mathrm{CH}\left(\mathrm{CH}_{3}\right)_{2}\right), 3.93\left(\mathrm{~m}, 1 \mathrm{H}, \mathrm{CH}\left(\mathrm{CH}_{3}\right)_{2}\right), 4.03\left(\mathrm{~m}, 1 \mathrm{H}, \mathrm{CH}\left(\mathrm{CH}_{3}\right)_{2}\right), 4.92(\mathrm{~d}, 1 \mathrm{H}, \mathrm{CH}), 6.88-8.05(16 \mathrm{H}, \mathrm{Ar}-$ $H)$ ppm. ${ }^{31} \mathrm{P}\{1 \mathrm{H}\}$ NMR $\left(121.50 \mathrm{MHz}, \mathrm{C}_{6} \mathrm{D}_{6}\right) \delta-13.47 \mathrm{ppm}$. Anal. calcd for $\mathrm{C}_{41} \mathrm{H}_{50} \mathrm{ClGeN}_{2} \mathrm{P}(709.84): \mathrm{C} 69.37, \mathrm{H}$ 7.10, N 3.95; Found: C 69.45, H 7.08, N, 3.34 .

11.4.47. Synthesis of $\mathrm{L}^{\prime \prime} \mathrm{GeB}\left(\mathrm{C}_{6} \mathrm{~F}_{5}\right)_{3}$ (48)

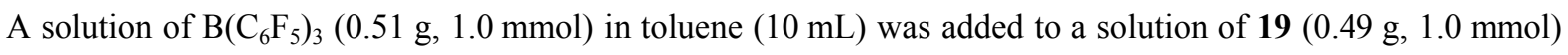
in toluene $(10 \mathrm{~mL})$ at room temperature. The brown-red color of the solution of $\mathbf{1 9}$ vanished slowly and pale yellow crystals of $\mathbf{4 8}$ were formed after two days at room temperature. Yield: $0.78 \mathrm{~g}(78 \%)$. Mp $265{ }^{\circ} \mathrm{C} .{ }^{1} \mathrm{H}$ NMR (500 MHz, THF-D 8 ): $\delta 7.37-7.44(\mathrm{~m}, 6 \mathrm{H}, \mathrm{Ar}-\mathrm{H}), 6.50$ (s, $1 \mathrm{H}, \gamma-\mathrm{CH}), 2.73$ (sept, $\left.2 \mathrm{H}, \mathrm{CH}\left(\mathrm{CH}_{3}\right)_{2}\right), 2.61$ (sept, 2H, $\left.\mathrm{CH}\left(\mathrm{CH}_{3}\right)_{2}\right), 2.47$ (s, $\left.2 \mathrm{H}, \mathrm{CH}_{2}\right), 1.84$ (s, 3H, $\left.\mathrm{CH}_{3}\right), 1.39$ (d, 6H, $\left.\mathrm{CH}\left(\mathrm{CH}_{3}\right)_{2}\right), 1.25$ (d, 6H, $\left.\mathrm{CH}\left(\mathrm{CH}_{3}\right)_{2}\right)$, $1.19\left(\mathrm{~d}, 6 \mathrm{H}, \mathrm{CH}\left(\mathrm{CH}_{3}\right)_{2}\right), 1.15\left(\mathrm{~d}, 6 \mathrm{H}, \mathrm{CH}\left(\mathrm{CH}_{3}\right)_{2}\right) \mathrm{ppm} .{ }^{11} \mathrm{~B}\left\{{ }^{1} \mathrm{H}\right\}$ NMR $\left(96.29 \mathrm{MHz}\right.$, THF-D $\left.\mathrm{D}_{8}\right): \delta-14.82 .{ }^{19} \mathrm{~F}\left\{{ }^{1} \mathrm{H}\right\}$ NMR (188.31 MHz, THF-D 8 ): $\delta-131.60$ (d, 6F, $o$-F), -163.40 (t, 6F, $m$-F), -167.28 (t, 3F, $p$-F). EI-MS (70 $\mathrm{eV}): m / z(\%): 475(100)\left[\mathrm{M}-\left(\mathrm{B}\left(\mathrm{C}_{6} \mathrm{~F}_{5}\right)_{3}, \mathrm{Me}\right)\right]^{+}$. Anal. calcd for $\mathrm{C}_{47} \mathrm{H}_{40} \mathrm{BF}_{15} \mathrm{GeN}_{2}(1001.26)$ : C 56.38, H 4.03, N 2.80; Found: C 56.27, H 3.82, N 2.68.

11.4.48. Synthesis of $\mathrm{L}^{\prime \prime \prime} \mathrm{GeB}\left(\mathrm{C}_{6} \mathrm{~F}_{5}\right)_{3}(\mathbf{4 9})$

A solution of 1,3-di-tert-butylimidazol-2-ylidene $(0.18 \mathrm{~g}, 1.0 \mathrm{mmol})$ in toluene $(10 \mathrm{~mL})$ was added to a solution of $48(0.49 \mathrm{~g}, 1.0 \mathrm{mmol})$ in toluene $(10 \mathrm{~mL})$ at room temperature. Immediately the reaction mixture turns to red in color. After $10 \mathrm{~min}$ the solvent was removed in vacuo and the title compound 49 was isolated as a red oil. ${ }^{1} \mathrm{H}$ NMR $\left(500 \mathrm{MHz}, \mathrm{C}_{6} \mathrm{D}_{6}\right): \delta 7.32(\mathrm{~s}, 1 \mathrm{H}, \mathrm{NCH}), 6.99-7.20(\mathrm{~m}, 6 \mathrm{H}, \mathrm{Ar}-H), 6.33(\mathrm{~s}, 2 \mathrm{H}, \mathrm{NCH}), 5.87(\mathrm{~s}, 1 \mathrm{H}, \gamma-\mathrm{CH}$ ), 3.86 (sept, $\left.2 \mathrm{H}, \mathrm{CH}\left(\mathrm{CH}_{3}\right)_{2}\right), 3.63(\mathrm{~s}, 1 \mathrm{H}, \mathrm{CH}), 3.60$ (sept, $\left.2 \mathrm{H}, \mathrm{CH}\left(\mathrm{CH}_{3}\right)_{2}\right), 2.96$ (s, $\left.1 \mathrm{H}, \mathrm{CH}\right), 2.45\left(\mathrm{~s}, 2 \mathrm{H}, \mathrm{CH}_{2}\right.$ ), $1.70\left(\mathrm{~d}, 6 \mathrm{H}, \mathrm{CH}\left(\mathrm{CH}_{3}\right)_{2}\right), 1.35\left(\mathrm{~d}, 12 \mathrm{H}, \mathrm{CH}\left(\mathrm{CH}_{3}\right)_{2}\right), 1.32$ (d, 6H, $\left.\mathrm{CH}\left(\mathrm{CH}_{3}\right)_{2}\right), 0.75\left(\mathrm{~s}, 18 \mathrm{H}, \mathrm{C}\left(\mathrm{CH}_{3}\right)_{3}\right) \mathrm{ppm}$. ${ }^{11} \mathrm{~B}\left\{{ }^{1} \mathrm{H}\right\}$ NMR (96.29 MHz, $\left.\mathrm{C}_{6} \mathrm{D}_{6}\right): \delta-13.66 .{ }^{19} \mathrm{~F}\left\{{ }^{1} \mathrm{H}\right\}$ NMR (188.31 MHz, $\left.\mathrm{C}_{6} \mathrm{D}_{6}\right): \delta-130.65$ (br, 6F, o-F), $163.77(\mathrm{t}, 6 \mathrm{~F}, m-\mathrm{F}),-167.32$ (br, 3F, $p$-F). From analytical data we do not obtain satisfactory results.

11.4.49. Synthesis of $\mathrm{L}^{\prime} \mathrm{Si}(\mathrm{H}) \mathrm{NH}_{2}$ (51)

Dry ammonia gas was added to a yellow solution of $\mathbf{5 0}(0.45 \mathrm{~g}, 1 \mathrm{mmol})$ in toluene $(20 \mathrm{~mL})$ at room temperature. The reaction mixture became colorless. Then the ammonia gas was bubbled through the solution for additional $15 \mathrm{~min}$. After that the solvent was removed in vacuo and the residue was extracted with $n$-hexane $(30 \mathrm{~mL})$. The solution was reduced to half of the volume. Storage of the solution at $-32{ }^{\circ} \mathrm{C}$ in a freezer for two days yielded colorless crystals of $\mathbf{5 1}$, which are suitable for single crystal X-ray diffraction studies. Yield: $0.41 \mathrm{~g}$ (90\%); Mp $223{ }^{\circ} \mathrm{C} .{ }^{1} \mathrm{H}$ NMR (500 MHz, $\left.\mathrm{C}_{6} \mathrm{D}_{6}\right): \delta=7.02-7.22(\mathrm{~m}, 6 \mathrm{H}, \mathrm{Ar}-H), 5.31(\mathrm{~s}, 1 \mathrm{H}, \gamma-\mathrm{CH}), 4.97(\mathrm{t}, 1 \mathrm{H}$, $\mathrm{Si}-H$ ), 3.93 (s, $1 \mathrm{H}, \mathrm{CH}_{2}$ ), 3.62 (sept, $\left.2 \mathrm{H}, \mathrm{CH}\left(\mathrm{CH}_{3}\right)_{2}\right), 3.59$ (sept, $\left.2 \mathrm{H}, \mathrm{CH}\left(\mathrm{CH}_{3}\right)_{2}\right), 3.35$ (s, $\left.1 \mathrm{H}, \mathrm{CH}_{2}\right), 1.49$ (s, $3 \mathrm{H}$, $\left.\mathrm{CH}_{3}\right), 1.40\left(\mathrm{~d}, 6 \mathrm{H}, \mathrm{CH}\left(\mathrm{CH}_{3}\right)_{2}\right), 1.22-1.18\left(\mathrm{~m}, 18 \mathrm{H}, \mathrm{CH}\left(\mathrm{CH}_{3}\right)_{2}\right), 0.35$ (br, $\left.2 \mathrm{H}, \mathrm{NH}_{2}\right) \mathrm{ppm}$. IR (Nujol, $\left.\mathrm{KBr}\right): \widetilde{v}=$ 3477, and $3382(\mathrm{~N}-\mathrm{H})$, and $2633(\mathrm{Si}-\mathrm{H}) \mathrm{cm}^{-1}$. EI-MS (70 eV): $\mathrm{m} / z(\%): 446(100)$ [M-Me] $]^{+}$. Anal. calcd for $\mathrm{C}_{29} \mathrm{H}_{43} \mathrm{~N}_{3} \mathrm{Si}$ (461.76): C 75.43, H 9.39, N 9.10; Found: C 75.10, H 9.41, N 9.14.

11.4.50. Synthesis of $\mathrm{L}^{\prime} \mathrm{Si}(\mathrm{H}) \mathrm{NHNH}_{2}$ (52)

$\mathrm{NH}_{2} \mathrm{NH}_{2}\left(1 \mathrm{~mL}, 1 \mathrm{M}\right.$ in THF) was added to a yellow solution of $\mathbf{5 0}(0.45 \mathrm{~g}, 1 \mathrm{mmol})$ in toluene $(20 \mathrm{~mL})$ at $0{ }^{\circ} \mathrm{C}$. Then the reaction mixture was allowed to come to room temperature. After that the reaction mixture was stirred for additional $30 \mathrm{~min}$. All the volatiles were removed in vacuo and the residue was extracted with $n$-hexane (30 $\mathrm{mL}$ ). The solution was reduced to half of the volume. Storage of the solution at $-32{ }^{\circ} \mathrm{C}$ in a freezer for two days 
yielded 52 as colorless crystals suitable for single crystal X-ray diffraction studies. Yield: $0.40 \mathrm{~g} \mathrm{(85 \% ).} \mathrm{Mp} 177$ ${ }^{\circ} \mathrm{C} .{ }^{1} \mathrm{H}$ NMR $\left(500 \mathrm{MHz}, \mathrm{C}_{6} \mathrm{D}_{6}\right): \delta 7.03-7.19(\mathrm{~m}, 6 \mathrm{H}, \mathrm{Ar}-H), 5.30(\mathrm{~s}, 1 \mathrm{H}, \gamma-\mathrm{CH}), 5.05(\mathrm{~d}, 1 \mathrm{H}, \mathrm{Si}-H), 3.94(\mathrm{~s}, 1 \mathrm{H}$, $\mathrm{CH}_{2}$ ), 3.65 (sept, $\left.2 \mathrm{H}, \mathrm{CH}\left(\mathrm{CH}_{3}\right)_{2}\right), 3.55$ (sept, $\left.2 \mathrm{H}, \mathrm{CH}\left(\mathrm{CH}_{3}\right)_{2}\right), 3.30$ (s, $\left.1 \mathrm{H}, \mathrm{CH}_{2}\right), 2.41$ (br, $\left.1 \mathrm{H}, \mathrm{NH}\right), 1.85$ (d, $2 \mathrm{H}$, $\left.\mathrm{NH} H_{2}\right), 1.47$ (s, $\left.3 \mathrm{H}, \mathrm{CH}_{3}\right), 1.39-1.17\left(\mathrm{~m}, 24 \mathrm{H}, \mathrm{CH}\left(\mathrm{CH}_{3}\right)_{2}\right) \mathrm{ppm} .{ }^{29} \mathrm{Si} \mathrm{NMR}\left(99.35 \mathrm{~Hz}, \mathrm{C}_{6} \mathrm{D}_{6}\right): \delta-43.63\left(\mathrm{dd},{ }^{2} J(\mathrm{Si}-\mathrm{H})\right.$ $\left.=260.5 \mathrm{~Hz},{ }^{3} J(\mathrm{Si}-\mathrm{H})=12.73 \mathrm{~Hz}\right) \mathrm{ppm}$. EI-MS $(70 \mathrm{eV}): \mathrm{m} / \mathrm{z}(\%): 461(100)[\mathrm{M}-\mathrm{Me}]^{+}$. Anal. calcd for $\mathrm{C}_{29} \mathrm{H}_{44} \mathrm{~N}_{4} \mathrm{Si}$ (476.33): C 73.06, H 9.30, N 11.75; Found: C 72.47, H 9.14, N 11.65.

\subsubsection{Synthesis of L'Si(H)NHNHMe (53)}

$\mathrm{MeNHNH}_{2}(0.05 \mathrm{~g}, 1 \mathrm{mmol})$ was added to a yellow solution of $\mathbf{5 0}(0.45 \mathrm{~g}, 1 \mathrm{mmol})$ in toluene $(20 \mathrm{~mL})$ at room temperature. After that the reaction mixture was stirred for additional $30 \mathrm{~min}$. All the volatiles were removed in vacuo and the residue was extracted with $n$-hexane $(30 \mathrm{~mL})$. Yield: $0.39 \mathrm{~g}(80 \%)$. Mp $73{ }^{\circ} \mathrm{C} .{ }^{1} \mathrm{H}$ NMR $(500$ $\left.\mathrm{MHz}, \mathrm{C}_{6} \mathrm{D}_{6}\right): \delta 7.04-7.22(\mathrm{~m}, 6 \mathrm{H}, \mathrm{Ar}-H), 5.34(\mathrm{~d}, 1 \mathrm{H}, \mathrm{Si}-H), 5.33(\mathrm{~s}, 1 \mathrm{H}, \gamma-\mathrm{CH}), 3.99$ (s, $\left.1 \mathrm{H}, \mathrm{CH}_{2}\right), 3.71$ (sept, $\left.1 \mathrm{H}, \mathrm{CH}\left(\mathrm{CH}_{3}\right)_{2}\right), 3.65$ (sept, $\left.1 \mathrm{H}, \mathrm{CH}\left(\mathrm{CH}_{3}\right)_{2}\right), 3.59$ (sept, $\left.1 \mathrm{H}, \mathrm{CH}\left(\mathrm{CH}_{3}\right)_{2}\right), 3.50$ (sept, $\left.1 \mathrm{H}, \mathrm{CH}\left(\mathrm{CH}_{3}\right)_{2}\right), 3.35$ (s, $1 \mathrm{H}$, $\left.\mathrm{CH}_{2}\right), 2.25$ (d, 1H, $\mathrm{SiNH}$ ), 1.73 (q, $\left.1 \mathrm{H}, \mathrm{NHCH}_{3}\right), 1.66$ (br, 3H, NCH$)_{3}, 1.49$ (s, 3H, $\left.\mathrm{CH}_{3}\right), 1.42$ (d, 3H, $\left.\mathrm{CH}\left(\mathrm{CH}_{3}\right)_{2}\right), 1.38\left(\mathrm{~d}, 3 \mathrm{H}, \mathrm{CH}\left(\mathrm{CH}_{3}\right)_{2}\right), 1.37\left(\mathrm{~d}, 3 \mathrm{H}, \mathrm{CH}\left(\mathrm{CH}_{3}\right)_{2}\right), 1.13-1.31$ (m, $\left.15 \mathrm{H}, \mathrm{CH}\left(\mathrm{CH}_{3}\right)_{2}\right)$ ppm. ${ }^{29} \mathrm{Si} \mathrm{NMR}$ $\left(99.35 \mathrm{~Hz}, \mathrm{C}_{6} \mathrm{D}_{6}\right): \delta-43.64\left(\mathrm{ddd},{ }^{2} J(\mathrm{Si}-\mathrm{H})=263.8 \mathrm{~Hz},{ }^{3} J(\mathrm{Si}-\mathrm{H})=13.17 \mathrm{~Hz},{ }^{4} J(\mathrm{Si}-\mathrm{H})=2.34 \mathrm{~Hz}\right)$ ppm. EI-MS $(70$ eV): $m / z$ (\%): 475 (100) [M-Me] ${ }^{+}$. Anal. calcd for $\mathrm{C}_{30} \mathrm{H}_{46} \mathrm{~N}_{4} \mathrm{Si}$ (490.35): C 73.42, H 9.45, N 11.42; Found: C 73.74, H 9.34, N 10.48 .

\subsubsection{Synthesis of 54}

A solution of $\mathrm{Ph}_{2} \mathrm{C}=\mathrm{NNH}_{2}(0.20 \mathrm{~g}, 1 \mathrm{mmol})$ in toluene $(5 \mathrm{~mL})$ was added to a yellow solution of $\mathbf{5 0}(0.45 \mathrm{~g}, 1$ $\mathrm{mmol})$ in toluene $(20 \mathrm{~mL})$ at room temperature. After that the reaction mixture was stirred for additional $1 \mathrm{~h}$. All the volatiles were removed in vacuo and the residue was extracted with $n$-hexane $(30 \mathrm{~mL})$. Suitable crystals for $\mathrm{X}$-ray structural analysis are obtained from a hot saturated $n$-hexane solution of $\mathbf{5 4}$ after keeping the solution overnight at room temperature. Yield: $0.41 \mathrm{~g}(90 \%)$. Mp $192{ }^{\circ} \mathrm{C} .{ }^{1} \mathrm{H}$ NMR $\left(500 \mathrm{MHz}, \mathrm{C}_{6} \mathrm{D}_{6}\right): \delta 7.00-7.17$ (m, $6 \mathrm{H}, \mathrm{Ar}-H), 6.04(\mathrm{~d}, 1 \mathrm{H}, \mathrm{CH}), 5.67(\mathrm{~m}, 1 \mathrm{H}, \mathrm{CH}), 5.56(\mathrm{~s}, 1 \mathrm{H}, \gamma-\mathrm{CH}), 5.52(\mathrm{~m}, 1 \mathrm{H}, \mathrm{CH}), 5.41(\mathrm{~m}, 1 \mathrm{H}, \mathrm{CH}), 4.02$ (s, $\left.1 \mathrm{H}, \mathrm{CH})_{2}\right), 3.88\left(\mathrm{sept}, 1 \mathrm{H}, \mathrm{CH}\left(\mathrm{CH}_{3}\right)_{2}\right), 3.74$ (sept, $\left.1 \mathrm{H}, \mathrm{CH}\left(\mathrm{CH}_{3}\right)_{2}\right), 3.57$ (sept, $\left.2 \mathrm{H}, \mathrm{CH}\left(\mathrm{CH}_{3}\right)_{2}\right), 3.43(\mathrm{~s}, 1 \mathrm{H}$, $\left.\mathrm{CH}_{2}\right), 3.08$ (m, 1H, $\mathrm{SiCH}$ ), 2.97 (s, 2H, NH $\left.H_{2}\right), 1.54$ (s, 3H, $\left.\mathrm{CH}_{3}\right), 1.42$ (d, 3H, $\left.\mathrm{CH}\left(\mathrm{CH}_{3}\right)_{2}\right), 1.40$ (d, 3H, $\left.\mathrm{CH}\left(\mathrm{CH}_{3}\right)_{2}\right), 1.38$ (d, 3H, $\left.\mathrm{CH}\left(\mathrm{CH}_{3}\right)_{2}\right), 1.31$ (d, 3H, $\left.\mathrm{CH}\left(\mathrm{CH}_{3}\right)_{2}\right), 1.30$ (d, 3H, $\left.\mathrm{CH}\left(\mathrm{CH}_{3}\right)_{2}\right), 1.28$ (d, 3H, $\left.\mathrm{CH}\left(\mathrm{CH}_{3}\right)_{2}\right)$, $1.23\left(\mathrm{~d}, 3 \mathrm{H}, \mathrm{CH}\left(\mathrm{CH}_{3}\right)_{2}\right), 1.20$ (d, 3H, $\left.\mathrm{CH}\left(\mathrm{CH}_{3}\right)_{2}\right)$ ppm. ${ }^{29} \mathrm{Si} \mathrm{NMR}\left(99.35 \mathrm{~Hz}, \mathrm{C}_{6} \mathrm{D}_{6}\right): \delta-19.97\left(\mathrm{~d},{ }^{3} J(\mathrm{Si}-\mathrm{H})=\right.$ 20.4Hz) ppm. EI-MS (70 eV): $m / z(\%): 625(100)[\mathrm{M}-\mathrm{Me}]^{+}$. Anal. calcd for $\mathrm{C}_{42} \mathrm{H}_{52} \mathrm{~N}_{4} \mathrm{Si}$ (640.97): C 78.70, $\mathrm{H}$ 8.18, N 8.74; Found: C 77.18, H 8.01, N 8.44.

\subsubsection{Synthesis of $\mathrm{LGeNHNCHCO}_{2} \mathrm{Et}$ (55)}

A solution of ethyl diazoacetate $(0.27 \mathrm{~g}, 2.4 \mathrm{mmol})$ in toluene $(10 \mathrm{~mL})$ was slowly added drop by drop to a stirred solution of $5(0.98 \mathrm{~g}, 2 \mathrm{mmol})$ in toluene $(20 \mathrm{~mL})$ at room temperature. During this time the color of the reaction mixture changed from red to yellowish. The reaction mixture was stirred for additional $30 \mathrm{~min}$. After removal of all the volatiles, the residue was extracted with toluene $(20 \mathrm{~mL})$ and concentrated to about $10 \mathrm{~mL}$ and stored at room temperature. Yellow crystals of 55 are formed after two days. Yield: $1.15 \mathrm{~g}(95 \%)$. Mp 160 ${ }^{\circ} \mathrm{C} .{ }^{1} \mathrm{H}$ NMR $\left(500 \mathrm{MHz}, \mathrm{C}_{6} \mathrm{D}_{6}\right): \delta 7.25(\mathrm{br}, 1 \mathrm{H}, \mathrm{N} H), 7.05-7.15(\mathrm{~m}, 6 \mathrm{H}, \mathrm{Ar}-H), 6.62(\mathrm{~s}, 1 \mathrm{H}, \mathrm{CH}), 4.91(\mathrm{~s}, 1 \mathrm{H}, \gamma-$ $\mathrm{CH}$ ), 4.11 (q, 2H, $\mathrm{CH}_{2} \mathrm{CH}_{3}$ ), 3.27 (sept, 2H, $\left.\mathrm{CH}\left(\mathrm{CH}_{3}\right)_{2}\right), 3.16$ (sept, 2H, $\left.\mathrm{CH}\left(\mathrm{CH}_{3}\right)_{2}\right), 1.56$ (s, 6H, $\left.\mathrm{CH}_{3}\right), 1.27$ (d, $\left.6 \mathrm{H}, \mathrm{CH}\left(\mathrm{CH}_{3}\right)_{2}\right), 1.13\left(\mathrm{~d}, 12 \mathrm{H}, \mathrm{CH}\left(\mathrm{CH}_{3}\right)_{2}\right), 1.07$ (d, 6H, $\left.\mathrm{CH}\left(\mathrm{CH}_{3}\right)_{2}\right), 1.03$ (t, 3H, $\left.\mathrm{CH}_{2} \mathrm{CH}_{3}\right)$ ppm. IR (Nujol, $\mathrm{KBr}$, $\left.\mathrm{cm}^{-1}\right): \widetilde{v}=3188(\mathrm{~N}-\mathrm{H}), 2726(\mathrm{C}-\mathrm{H}), 1684(\mathrm{C}=\mathrm{O})$. EI-MS $(70 \mathrm{eV}): \mathrm{m} / z(\%): 491(100)$ [M-NHNCHCO $\left.{ }_{2} \mathrm{Et}\right]^{+}$. Anal. calcd for $\mathrm{C}_{33} \mathrm{H}_{48} \mathrm{GeN}_{4} \mathrm{O}_{2}$ (605.40): C 65.47, H 7.99, N 9.25; Found: C 65.34, H 8.53, N 9.37.

\subsubsection{Synthesis of $\mathrm{LGeNHNCHSiMe}_{3}$ (56)}

In a dry box an excess solution of trimethylsilyl diazomethane $(1.5 \mathrm{~mL}, \sim 2 \mathrm{M}$, in $n$-hexane) was slowly added drop by drop to a schlenk flask containing $5(0.98 \mathrm{~g}, 2 \mathrm{mmol})$ in $10 \mathrm{~mL} n$-hexane. Then the flask was kept at room temperature for one day and after that the yellow crystals of $\mathbf{5 6}$ are formed nearly quantitatively, which are suitable for X-ray structural analysis. Yield: $1.10 \mathrm{~g}(90 \%)$. Mp $153{ }^{\circ} \mathrm{C} .{ }^{1} \mathrm{H}$ NMR $\left(500 \mathrm{MHz}, \mathrm{C}_{6} \mathrm{D}_{6}\right): \delta 7.02-7.15$ 
$(\mathrm{m}, 6 \mathrm{H}, \mathrm{Ar}-H), 6.44(\mathrm{~d}, 1 \mathrm{H}, \mathrm{CH}), 6.37(\mathrm{~d}, 1 \mathrm{H}, \mathrm{NH}), 4.96(\mathrm{~s}, 1 \mathrm{H}, \gamma-\mathrm{CH}), 3.47\left(\mathrm{sept}, 2 \mathrm{H}, \mathrm{CH}\left(\mathrm{CH}_{3}\right)_{2}\right), 3.30$ (sept, $\left.2 \mathrm{H}, \mathrm{CH}\left(\mathrm{CH}_{3}\right)_{2}\right), 1.63\left(\mathrm{~s}, 6 \mathrm{H}, \mathrm{CH}_{3}\right), 1.36\left(\mathrm{~d}, 6 \mathrm{H}, \mathrm{CH}\left(\mathrm{CH}_{3}\right)_{2}\right), 1.25\left(\mathrm{~d}, 12 \mathrm{H}, \mathrm{CH}\left(\mathrm{CH}_{3}\right)_{2}\right), 1.15\left(\mathrm{~d}, 6 \mathrm{H}, \mathrm{CH}(\mathrm{CH})_{2}\right)$, $1.14\left(\mathrm{~d}, 6 \mathrm{H}, \mathrm{CH}\left(\mathrm{CH}_{3}\right)_{2}\right), 0.25\left(\mathrm{~s}, 9 \mathrm{H}, \mathrm{Si}\left(\mathrm{CH}_{3}\right)_{3}\right) \mathrm{ppm} .{ }^{29} \mathrm{Si}\left\{{ }^{1} \mathrm{H}\right\} \mathrm{NMR}\left(125.77 \mathrm{~Hz}, \mathrm{C}_{6} \mathrm{D}_{6}\right): \delta 6.28\left(\mathrm{Si}\left(\mathrm{CH}_{3}\right)_{3}\right) \mathrm{ppm}$. EI-MS (70 eV): $m / z$ (\%): 580 (100) [M-Me] $]^{+}$Anal. calcd for $\mathrm{C}_{33} \mathrm{H}_{52} \mathrm{GeN}_{4} \mathrm{Si}$ (605.52): C 65.46, H 8.66, N 9.25; Found: C 65.27, H 9.12, N 8.50.

11.4.55. Synthesis of $\mathrm{L}^{\prime} \mathrm{Ge}(\mathrm{Me})\left(\mathrm{NHNCHSiMe}_{3}\right)(\mathbf{5 7})$

A solution of trimethylsilyl diazomethane $(0.5 \mathrm{~mL}, 2 \mathrm{M}$, in $n$-hexane) was slowly added drop by drop to a Schlenk flask containing LGeMe $(0.50 \mathrm{~g}, 1 \mathrm{mmol})$ in $15 \mathrm{~mL} n$-hexane. The solution slowly changed the color from red to yellow. After constant stirring overnight at ambient temperature all volatiles were removed in vacuo, and the remaining residue was extracted with $n$-hexane $(15 \mathrm{~mL})$ and after removal of all the volatiles from the extract compound 57 was obtained as a yellow solid. Yield: $0.45 \mathrm{~g}(72 \%)$. Mp $103{ }^{\circ} \mathrm{C} .{ }^{1} \mathrm{H} \mathrm{NMR}(500 \mathrm{MHz}$, $\left.\mathrm{C}_{6} \mathrm{D}_{6}\right): \delta 7.02-7.14(\mathrm{~m}, 6 \mathrm{H}, \mathrm{Ar}-H), 6.76(\mathrm{~s}, 1 \mathrm{H}, \mathrm{NH}), 5.78(\mathrm{~s}, 1 \mathrm{H}, \gamma-\mathrm{CH}), 5.26(\mathrm{~s}, 1 \mathrm{H}, \mathrm{CH}), 3.83\left(\mathrm{~s}, 1 \mathrm{H}, \mathrm{CH}_{2}\right)$, 3.71 (sept, 1H, $\left.\mathrm{CH}\left(\mathrm{CH}_{3}\right)_{2}\right), 3.58$ (sept, 2H, $\left.\mathrm{CH}\left(\mathrm{CH}_{3}\right)_{2}\right), 3.51$ (sept, 1H, $\left.\mathrm{CH}\left(\mathrm{CH}_{3}\right)_{2}\right), 3.21$ (s, 1H, $\left.\mathrm{CH}_{2}\right), 1.54(\mathrm{~s}$, $\left.3 \mathrm{H}, \mathrm{CH}_{3}\right), 1.41\left(\mathrm{~d}, 3 \mathrm{H}, \mathrm{CH}\left(\mathrm{CH}_{3}\right)_{2}\right), 1.39$ (d, 3H, $\left.\mathrm{CH}\left(\mathrm{CH}_{3}\right)_{2}\right), 1.22-1-27\left(\mathrm{~m}, 12 \mathrm{H}, \mathrm{CH}\left(\mathrm{CH}_{3}\right)_{2}\right), 1.16(\mathrm{~d}, 3 \mathrm{H}$, $\left.\mathrm{CH}\left(\mathrm{CH}_{3}\right)_{2}\right), 1.05\left(\mathrm{~d}, 3 \mathrm{H}, \mathrm{CH}\left(\mathrm{CH}_{3}\right)_{2}\right), 0.17\left(\mathrm{~s}, 9 \mathrm{H}, \mathrm{Si}\left(\mathrm{CH}_{3}\right)_{3}\right) \mathrm{ppm} .{ }^{29} \mathrm{Si}\left\{{ }^{1} \mathrm{H}\right\} \mathrm{NMR}\left(125.77 \mathrm{~Hz}, \mathrm{C}_{6} \mathrm{D}_{6}\right): \delta-8.76$ $\left(\mathrm{Si}\left(\mathrm{CH}_{3}\right)_{3}\right)$ ppm. EI-MS (70 eV): $m / z(\%): 605$ (25) [M-Me] $]^{+}, 491$ (100) [M-Me, NHNCHTMS] ${ }^{+}$. Anal. calcd for $\mathrm{C}_{34} \mathrm{H}_{54} \mathrm{GeN}_{4} \mathrm{Si}$ (620.33): C 65.91, H 8.79; Found: C 65.81, H 9.14.

11.4.56. Synthesis of $\mathrm{LGeC}\left(\mathrm{N}_{2}\right) \mathrm{SiMe}_{3}(\mathbf{5 8})$

A solution of $\mathrm{N}_{2} \mathrm{CHSiMe}_{3}(0.7 \mathrm{~mL}, 2 \mathrm{M}$, in $n$-hexane $)$ was added to a solution of $19(0.49 \mathrm{~g}, 1.0 \mathrm{mmol})$ in $n$ hexane $(10 \mathrm{~mL})$ at room temperature. Then the flask was kept at room temperature for three days and after that yellow crystals of $\mathbf{5 8}$ were obtained, which are suitable for X-ray structural analysis. Yield: $0.53 \mathrm{~g}$ (88\%). Mp $244{ }^{\circ} \mathrm{C} .{ }^{1} \mathrm{H}$ NMR $\left(500 \mathrm{MHz}, \mathrm{C}_{6} \mathrm{D}_{6}\right): \delta$ 7.09-7.17 (m, 6H, Ar- $\left.H\right), 4.51(\mathrm{~s}, 1 \mathrm{H}, \gamma-\mathrm{CH}), 3.63$ (sept, 2H, $\left.\mathrm{CH}\left(\mathrm{CH}_{3}\right)_{2}\right), 3.60$ (sept, 2H, $\left.\mathrm{CH}\left(\mathrm{CH}_{3}\right)_{2}\right), 1.62(\mathrm{~s}, 6 \mathrm{H}, \mathrm{CH}), 1.58$ (d, 6H, $\left.\mathrm{CH}\left(\mathrm{CH}_{3}\right)_{2}\right), 1.43\left(\mathrm{~d}, 6 \mathrm{H}, \mathrm{CH}\left(\mathrm{CH}_{3}\right)_{2}\right)$, $1.25\left(\mathrm{~d}, 6 \mathrm{H}, \mathrm{CH}\left(\mathrm{CH}_{3}\right)_{2}\right), 1.13\left(\mathrm{~d}, 6 \mathrm{H}, \mathrm{CH}\left(\mathrm{CH}_{3}\right)_{2}\right),-0.07\left(\mathrm{Si}\left(\mathrm{CH}_{3}\right)_{3}\right) \mathrm{ppm} .{ }^{29} \mathrm{Si}\left\{{ }^{1} \mathrm{H}\right\} \mathrm{NMR}\left(99.35 \mathrm{~Hz}, \mathrm{C}_{6} \mathrm{D}_{6}\right): \delta-$ $2.43\left(\mathrm{Si}\left(\mathrm{CH}_{3}\right)_{3}\right)$ ppm EI-MS (70 eV): $\mathrm{m} / z(\%): 561$ (100) [M-iPr] ${ }^{+}$. Anal. calcd for $\mathrm{C}_{33} \mathrm{H}_{50} \mathrm{GeN}_{4} \mathrm{Si}(603.50): \mathrm{C}$ 65.68, H 8.35, N 9.28; Found: C 64.96, H 8.62, N 8.87.

11.4.57. Synthesis of $\mathrm{LGeN}(\mathrm{NC}) \mathrm{SiMe}_{3}(59)$

After keeping the supernatant solution of $\mathbf{5 8}$ for about 3 months at room temperature we observed the conversion of 58 to 59. $\mathrm{Mp} 210{ }^{\circ} \mathrm{C} .{ }^{1} \mathrm{H}$ NMR $\left(500 \mathrm{MHz}, \mathrm{C}_{6} \mathrm{D}_{6}\right): \delta 7.06-7.18(\mathrm{~m}, 6 \mathrm{H}, \mathrm{Ar}-H), 4.75(\mathrm{~s}, 1 \mathrm{H}, \gamma-\mathrm{CH})$, 3.89 (sept, 2H, $\mathrm{CH}\left(\mathrm{CH}_{3}\right)_{2}$ ), 3.39 (sept, 2H, CH(CH$\left.)_{2}\right), 1.66$ (s, 6H, $\left.\mathrm{CH}_{3}\right), 1.57$ (d, 6H, $\left.\mathrm{CH}\left(\mathrm{CH}_{3}\right)_{2}\right), 1.37$ (d, 6H, $\left.\mathrm{CH}\left(\mathrm{CH}_{3}\right)_{2}\right), 1.26\left(\mathrm{~d}, 6 \mathrm{H}, \mathrm{CH}\left(\mathrm{CH}_{3}\right)_{2}\right), 1.07\left(\mathrm{~d}, 6 \mathrm{H}, \mathrm{CH}\left(\mathrm{CH}_{3}\right)_{2}\right),-0.04\left(\mathrm{Si}\left(\mathrm{CH}_{3}\right)_{3}\right) \mathrm{ppm} .{ }^{29} \mathrm{Si}\left\{{ }^{1} \mathrm{H}\right\} \mathrm{NMR}(99.35 \mathrm{~Hz}$, $\left.\mathrm{C}_{6} \mathrm{D}_{6}\right): \delta 12.50\left(\mathrm{Si}\left(\mathrm{CH}_{3}\right)_{3}\right)$ ppm EI-MS (70 eV): $m / z(\%): 604(100)[\mathrm{M}]^{+}$.

11.4.58. Synthesis of $\mathrm{LSnNCNSiMe}_{3}(\mathbf{6 0})$

A solution of lithiotrimethylsilyldiazomethane $(4.0 \mathrm{mmol})$ prepared from a solution of trimethylsilyldiazomethane $(2.0 \mathrm{~mL}, 2 \mathrm{M}$ in hexane) and $n$-BuLi (2.5 mL, $1.6 \mathrm{~m}$ in hexane) in THF (15 mL) was added dropwise to the solution of $\mathrm{LSnCl}(2.20 \mathrm{~g}, 4.0 \mathrm{mmol})$ in $\mathrm{THF}(35 \mathrm{~mL})$ at $-78{ }^{\circ} \mathrm{C}$, and the solution was allowed to warmed up room temperature and stirred for another $30 \mathrm{~min}$. Solvents were removed in vacuo, and the residue was dissolved in $n$-hexane. The mixture was filtered, the solvent was partially removed, and $\mathbf{6 0}$ were obtained as a crystalline solid after keeping two days at $-30{ }^{\circ} \mathrm{C}$, which was also suitable for X-ray structural analysis. Yield: $2.16 \mathrm{~g}(60 \%)$. Mp $177{ }^{\circ} \mathrm{C} .{ }^{1} \mathrm{H}$ NMR (500 MHz, $\left.\mathrm{C}_{6} \mathrm{D}_{6}\right): \delta 7.02-7.15(\mathrm{~m}, 6 \mathrm{H}, \mathrm{Ar}-H), 4.96(\mathrm{~s}, 1 \mathrm{H}$, $\gamma-\mathrm{CH}$ ), 3.82 (sept, 2H, $\left.\mathrm{CH}\left(\mathrm{CH}_{3}\right)_{2}\right), 3.14$ (sept, 2H, $\left.\mathrm{CH}\left(\mathrm{CH}_{3}\right)_{2}\right), 1.61\left(\mathrm{~s}, 6 \mathrm{H}, \mathrm{CH}_{3}\right), 1.51\left(\mathrm{~d}, 6 \mathrm{H}, \mathrm{CH}\left(\mathrm{CH}_{3}\right)_{2}\right), 1.32$ $\left(\mathrm{d}, 12 \mathrm{H}, \mathrm{CH}\left(\mathrm{CH}_{3}\right)_{2}\right), 1.18\left(\mathrm{~d}, 6 \mathrm{H}, \mathrm{CH}\left(\mathrm{CH}_{3}\right)_{2}\right), 1.07$ (d, 6H, $\left.\mathrm{CH}\left(\mathrm{CH}_{3}\right)_{2}\right), 0.27\left(\mathrm{~s}, 9 \mathrm{H}, \mathrm{Si}\left(\mathrm{CH}_{3}\right)_{3}\right) \mathrm{ppm} .{ }^{29} \mathrm{Si}\left\{{ }^{1} \mathrm{H}\right\}$ NMR $\left(125.77 \mathrm{~Hz}, \mathrm{C}_{6} \mathrm{D}_{6}\right): \delta-6.05\left(\mathrm{Si}\left(\mathrm{CH}_{3}\right)_{3}\right) \mathrm{ppm} .{ }^{119} \mathrm{Sn}\left\{{ }^{1} \mathrm{H}\right\}$ NMR $\left(186.46 \mathrm{MHz}, \mathrm{C}_{6} \mathrm{D}_{6}\right): \delta-321.26$ ppm. EI-MS (70 eV): $m / z(\%): 649$ (100) [M] $]^{+}$. Anal. calcd for $\mathrm{C}_{33} \mathrm{H}_{50} \mathrm{~N}_{4} \mathrm{SiSn}$ (649.57): C 61.02, H 7.76, N 8.63; Found: C $61.42, \mathrm{H} 8.04, \mathrm{~N} 8.42$. 
11.4.59. Synthesis of $\mathrm{LGeNMe}_{2}(\mathbf{6 1})$

A solution of $\mathrm{LGeCl}(0.498 \mathrm{~g}, 1.0 \mathrm{mmol})$ in diethyl ether $(20 \mathrm{~mL})$ was added dropwise to a stirred suspension of $\mathrm{LiNMe}_{2}(0.051 \mathrm{~g}, 1.0 \mathrm{mmol})$ in diethyl ether $(10 \mathrm{~mL})$ at $-78{ }^{\circ} \mathrm{C}$. The reaction mixture was warmed to room temperature and was stirred for another $12 \mathrm{~h}$. The precipitate was filtered, and the solvent was partially reduced (ca. $20 \mathrm{~mL}$ ). Storage of the remaining solution in a $-32{ }^{\circ} \mathrm{C}$ freezer for overnight afforded colorless crystals of $\mathbf{6 1}$ suitable for X-ray diffraction analyses. Yield: $0.448 \mathrm{~g}(84 \%)$. Mp $225{ }^{\circ} \mathrm{C} .{ }^{1} \mathrm{H}$ NMR $\left(500 \mathrm{MHz}, \mathrm{C}_{6} \mathrm{D}_{6}\right): \delta 7.12-$ $7.18(\mathrm{~m}, 6 \mathrm{H}, \mathrm{Ar}-H), 4.82$ (s, $1 \mathrm{H}, \gamma-\mathrm{CH}), 3.43$ (sept, $\left.2 \mathrm{H}, \mathrm{CH}\left(\mathrm{CH}_{3}\right)_{2}\right), 3.37$ (sept, $\left.2 \mathrm{H}, \mathrm{CH}\left(\mathrm{CH}_{3}\right)_{2}\right), 2.82(\mathrm{~s}, 6 \mathrm{H}$, $\left.\mathrm{N}\left(\mathrm{CH}_{3}\right)_{2}\right), 1.59$ (s, 6H, $\left.\mathrm{CH}_{3}\right), 1.33$ (d, 6H, $\left.\mathrm{CH}\left(\mathrm{CH}_{3}\right)_{2}\right), 1.29$ (d, 6H, $\left.\mathrm{CH}\left(\mathrm{CH}_{3}\right)_{2}\right), 1.26$ (d, 6H, $\left.\mathrm{CH}\left(\mathrm{CH}_{3}\right)_{2}\right), 1.13$ (d, $\left.6 \mathrm{H}, \mathrm{CH}\left(\mathrm{CH}_{3}\right)_{2}\right)$ ppm. EI-MS (70 eV): $\mathrm{m} / z(\%): 491$ (100) [M-NMe $]^{+}$. Anal. calcd for $\mathrm{C}_{31} \mathrm{H}_{47} \mathrm{GeN}_{3}(534.36): \mathrm{C}$ 69.68, H 8.87, N 7.86; Found: C 69.82, H 9.54, N 7.80.

11.4.60. Synthesis of $\mathrm{LGe}(\mathrm{S}) \mathrm{NMe}_{2}(\mathbf{6 2})$

A solution of $61(0.530 \mathrm{~g}, 1.00 \mathrm{mmol})$ in toluene $(30 \mathrm{~mL})$ was slowly added to a suspension of elemental sulfur $(0.032 \mathrm{~g}, 1.00 \mathrm{mmol})$ in toluene $(15 \mathrm{~mL})$ by cannula at room temperature. After $0.5 \mathrm{~h}$ under constant stirring at ambient temperature the red solution turned slightly yellow. All volatiles were removed in vacuo, and the remaining residue was extracted with toluene $(15 \mathrm{~mL})$. The solvent was removed in vacuo to yield $\mathbf{6 2}$ as a slight yellow powder. Yield: $0.340 \mathrm{~g}(60 \%)$. Mp $230{ }^{\circ} \mathrm{C} .{ }^{1} \mathrm{H}$ NMR $\left(300 \mathrm{MHz}, \mathrm{C}_{6} \mathrm{D}_{6}\right): \delta=7.02-7.16(\mathrm{~m}, 6 \mathrm{H}, \mathrm{Ar}-\mathrm{H})$, 4.70 (s, $1 \mathrm{H}, \gamma-\mathrm{CH}$ ), 3.47 (sept, $\left.2 \mathrm{H}, \mathrm{CH}\left(\mathrm{CH}_{3}\right)_{2}\right), 3.15$ (sept, $\left.2 \mathrm{H}, \mathrm{CH}\left(\mathrm{CH}_{3}\right)_{2}\right), 2.89-2.73$ (br, 6H, N( $\left.\left(\mathrm{CH}_{3}\right)_{2}\right), 1.60$ (d, $\left.6 \mathrm{H}, \mathrm{CH}\left(\mathrm{CH}_{3}\right)_{2}\right), 1.44$ (s, 6H, $\left.\mathrm{CH}_{3}\right), 1.29$ (d, 6H, $\left.\mathrm{CH}\left(\mathrm{CH}_{3}\right)_{2}\right), 1.18$ (d, 6H, $\left.\mathrm{CH}\left(\mathrm{CH}_{3}\right)_{2}\right), 1.07\left(\mathrm{~d}, 6 \mathrm{H}, \mathrm{CH}\left(\mathrm{CH}_{3}\right)_{2}\right)$ ppm. EI-MS (70 eV): $m / z(\%): 523$ (100) [M-NMe $]^{+}$.

\subsubsection{Synthesis of LGeOTf (63)}

A solution of $\mathrm{LGeCl}(0.520 \mathrm{~g}, 1 \mathrm{mmol})$ in toluene $(15 \mathrm{~mL})$ was added drop by drop to a stirred suspension of $\mathrm{AgOSO}_{2} \mathrm{CF}_{3}(0.26 \mathrm{~g}, 1 \mathrm{mmol})$ in toluene $(10 \mathrm{~mL})$ at room remperature. The solution was stirred for additional 2 h. After filtration the filtrate was concentrated to about $10 \mathrm{~mL}$ and stored in a $-30{ }^{\circ} \mathrm{C}$ freezer. Colorless crystals of 63 were formed after two days. Yield: $0.575 \mathrm{~g}(90 \%)$. Mp $216^{\circ} \mathrm{C} .{ }^{1} \mathrm{H}$ NMR $\left(500 \mathrm{MHz}, \mathrm{C}_{6} \mathrm{D}_{6}\right): \delta 7.06-7.15$ (m, 6H, Ar- $H$ ), 5.58 (s, $1 \mathrm{H}, \gamma-\mathrm{CH}), 3.27$ (sept, 4H, $\left.\mathrm{CH}\left(\mathrm{CH}_{3}\right)_{2}\right), 1.68$ (s, 6H, $\left.\mathrm{CH}_{3}\right), 1.19$ (d, 12H, $\left.\mathrm{CH}\left(\mathrm{CH}_{3}\right)_{2}\right), 1.15$ (d, $\left.12 \mathrm{H}, \mathrm{CH}\left(\mathrm{CH}_{3}\right)_{2}\right)$ ppm. ${ }^{19} \mathrm{~F}\left\{{ }^{1} \mathrm{H}\right\}$ NMR $\left(188.28 \mathrm{MHz}, \mathrm{C}_{6} \mathrm{D}_{6}\right): \delta-76.5$ ppm. EI-MS: $m / z(\%) 640(100)[\mathrm{M}]^{+}$. Anal. calcd for $\mathrm{C}_{30} \mathrm{H}_{41} \mathrm{~F}_{3} \mathrm{GeN}_{2} \mathrm{O}_{3} \mathrm{~S}$ (640.20): C 56.36, H 6.46, N 4.38, S 5.02; Found C 56.55, H 6.93, N 4.35, S 5.02 .

11.4.62. Synthesis of LSnMe (64)

A solution of MeLi in diethyl ether $(0.63 \mathrm{~mL}, 1.6 \mathrm{M})$ was added drop by drop to a stirred solution of $\mathrm{LSnCl}$ $(0.570 \mathrm{~g}, 1 \mathrm{mmol})$ in diethyl ether $(25 \mathrm{~mL})$ at $-78^{\circ} \mathrm{C}$. The reaction mixture was warmed to room temperature and was stirred for an additional $3 \mathrm{~h}$. After removal of all the volatiles, the residue was extracted with $n$-hexane $(20 \mathrm{~mL})$ and concentrated to about $10 \mathrm{~mL}$ and stored in a $-30{ }^{\circ} \mathrm{C}$ freezer. Yellow crystals of 64 suitable for Xray diffraction analysis are formed after two days. Yield: $0.485 \mathrm{~g}(88 \%) . \mathrm{Mp} 170{ }^{\circ} \mathrm{C} .{ }^{1} \mathrm{H}$ NMR $(500 \mathrm{MHz}$, $\left.\mathrm{C}_{6} \mathrm{D}_{6}\right): \delta 7.10-7.15(\mathrm{~m}, 6 \mathrm{H}, \mathrm{Ar}-H), 4.86(\mathrm{~s}, 1 \mathrm{H}, \gamma-\mathrm{CH}), 3.59$ (sept, $\left.2 \mathrm{H}, \mathrm{CH}\left(\mathrm{CH}_{3}\right)_{2}\right), 3.37$ (sept, $\left.2 \mathrm{H}, \mathrm{CH}\left(\mathrm{CH}_{3}\right)_{2}\right)$, 1.60 (s, 6H, $\left.\mathrm{CH}_{3}\right), 1.35$ (d, 6H, $\left.\mathrm{CH}\left(\mathrm{CH}_{3}\right)_{2}\right), 1.22$ (d, 6H, $\left.\mathrm{CH}\left(\mathrm{CH}_{3}\right)_{2}\right), 1.19$ (d, $\left.12 \mathrm{H}, \mathrm{CH}\left(\mathrm{CH}_{3}\right)_{2}\right), 0.59$ (t, 3H, Sn$\left.\mathrm{CH}_{3}\right)$ ppm. ${ }^{119} \mathrm{Sn}\left\{{ }^{1} \mathrm{H}\right\}$ NMR $\left(186.50 \mathrm{MHz}, \mathrm{C}_{6} \mathrm{D}_{6}\right): \delta 192.65$ ppm. EI-MS: $m / z(\%) 537$ (100) [M-Me $]^{+}$. Anal. calcd for $\mathrm{C}_{30} \mathrm{H}_{44} \mathrm{~N}_{2} \mathrm{Sn}$ (552.25): C 65.35, H 8.04, N 5.08; Found C 64.96, H 8.64, N 5.09.

11.4.63. Synthesis of $\mathrm{LSnN}\left(\mathrm{SiMe}_{3}\right)_{2}$ (65)

A solution of LH $(0.418 \mathrm{~g}, 1 \mathrm{mmol})$ in THF $(15 \mathrm{~mL})$ was added drop by drop to a stirred suspension of $\mathrm{KN}\left(\mathrm{SiMe}_{3}\right)_{2}(0.400 \mathrm{~g}, 2 \mathrm{mmol})$ in THF $(10 \mathrm{~mL})$ at room temperature and stirring was continued overnight. The resulting solution was added drop by drop to a stirred suspension of $\mathrm{SnCl}_{2}(0.190 \mathrm{~g}, 1 \mathrm{mmol})$ in THF $(20 \mathrm{~mL})$ at room temperature under stirring for another $24 \mathrm{~h}$. After removal of all the volatiles, the residue was extracted with $n$-hexane $(30 \mathrm{~mL})$ and concentrated to about $5 \mathrm{~mL}$ and finally stored in a $0{ }^{\circ} \mathrm{C}$ freezer. Yellow crystals of 65 suitable for X-ray diffraction analysis are formed after one day. Yield: $0.570 \mathrm{~g}(82 \%)$. Mp $202{ }^{\circ} \mathrm{C} .{ }^{1} \mathrm{H}$ NMR ( $\left.300 \mathrm{MHz}, \mathrm{C}_{6} \mathrm{D}_{6}\right): \delta 6.98-7.21(\mathrm{~m}, 6 \mathrm{H}, \mathrm{Ar}-H), 5.01(\mathrm{~s}, 1 \mathrm{H}, \gamma-\mathrm{CH}), 3.73\left(\mathrm{sept}, 2 \mathrm{H}, \mathrm{CH}\left(\mathrm{CH}_{3}\right)_{2}\right), 3.16$ (sept, $2 \mathrm{H}$, $\left.\mathrm{CH}\left(\mathrm{CH}_{3}\right)_{2}\right), 1.52\left(\mathrm{~s}, 6 \mathrm{H}, \mathrm{CH}_{3}\right), 1.45\left(\mathrm{~d}, 6 \mathrm{H}, \mathrm{CH}\left(\mathrm{CH}_{3}\right)_{2}\right), 1.22\left(\mathrm{q}, 12 \mathrm{H}, \mathrm{CH}\left(\mathrm{CH}_{3}\right)_{2}\right), 1.13\left(\mathrm{~d}, 6 \mathrm{H}, \mathrm{CH}\left(\mathrm{CH}_{3}\right)_{2}\right), 0.46$ (s, 9H, $\left.\mathrm{Si}\left(\mathrm{CH}_{3}\right)_{3}\right), 0.24$ (s, 9H, $\left.\mathrm{Si}\left(\mathrm{CH}_{3}\right)_{3}\right)$ ppm. ${ }^{29} \mathrm{Si}\left\{{ }^{1} \mathrm{H}\right\} \mathrm{NMR}\left(99.36 \mathrm{~Hz}, \mathrm{C}_{6} \mathrm{D}_{6}\right): \delta 0.61\left(\mathrm{SiMe}_{3}\right),-4.70\left(\mathrm{SiMe}_{3}\right)$ 
ppm. ${ }^{119} \mathrm{Sn}\left\{{ }^{1} \mathrm{H}\right\}$ NMR $\left(112.00 \mathrm{~Hz}, \mathrm{C}_{6} \mathrm{D}_{6}\right): \delta-134.54$ ppm. EI-MS (70 eV): $m / z(\%): 682$ (100) [M-Me] $]^{+}$Anal. Calcd for $\mathrm{C}_{35} \mathrm{H}_{59} \mathrm{~N}_{3} \mathrm{SnSi}_{2}(697)$ : C 60.33, H 8.54, N 6.03; Found C 60.20, H 8.49, N 5.88.

11.4.64. Synthesis of $\mathrm{LSnNMe}_{2}$ (66)

A solution of $\mathrm{LSnCl}(0.570 \mathrm{~g}, 1.0 \mathrm{mmol})$ in diethyl ether $(20 \mathrm{~mL})$ was added dropwise to a stirred suspension of $\mathrm{LiNMe}_{2}(0.051 \mathrm{~g}, 1.0 \mathrm{mmol})$ in diethyl ether $(10 \mathrm{~mL})$ at $-78{ }^{\circ} \mathrm{C}$. The reaction mixture was warmed to room temperature and was stirred for another $12 \mathrm{~h}$. The precipitate was filtered, and the solvent was partially reduced (ca. $20 \mathrm{~mL}$ ). Keepimg the flask in a $-32{ }^{\circ} \mathrm{C}$ freezer for overnight afforded title compound 66. Yield: $0.533 \mathrm{~g}$ (92\%). ${ }^{1} \mathrm{H}$ NMR (200 MHz, $\left.\mathrm{C}_{6} \mathrm{D}_{6}\right): \delta 7.02-7.16(\mathrm{~m}, 6 \mathrm{H}, \mathrm{Ar}-H)$ ), $4.82(\mathrm{~s}, 1 \mathrm{H}, \gamma-\mathrm{CH}), 3.45$ (sept, $\left.2 \mathrm{H}, \mathrm{CH}\left(\mathrm{CH}_{3}\right)_{2}\right)$, 3.37 (sept, $\left.2 \mathrm{H}, \mathrm{CH}\left(\mathrm{CH}_{3}\right)_{2}\right), 3.00$ (s, $\left.6 \mathrm{H}, \mathrm{N}\left(\mathrm{CH}_{3}\right)_{2}\right), 1.65$ (s, $\left.6 \mathrm{H}, \mathrm{CH}_{3}\right), 1.27-1.35$ (m, 18H, $\left.\mathrm{CH}\left(\mathrm{CH}_{3}\right)_{2}\right), 1.17$ (d, $\left.6 \mathrm{H}, \mathrm{CH}\left(\mathrm{CH}_{3}\right)_{2}\right) \mathrm{ppm}$.

11.4.65. Synthesis of $\mathrm{LSnOCPh}(2-\mathrm{Py})\left(\mathrm{NMe}_{2}\right)(67)$

A solution of 2-benzol pyridine ( $0.180 \mathrm{~g}, 1.00 \mathrm{mmol}$ in $5 \mathrm{~mL}$ toluene) was added by cannula to a solution of $\mathbf{6 6}$ $(0.540 \mathrm{~g}, 1.00 \mathrm{mmol}$ in toluene $20 \mathrm{~mL})$ at room temperature. After $12 \mathrm{~h}$ all volatiles were removed in vacuo, and the remaining residue was extracted with $n$-hexane $(25 \mathrm{~mL})$ and concentrated to about $15 \mathrm{~mL}$ and stored in a -30 ${ }^{\circ} \mathrm{C}$ freezer. Yellow crystals of $\mathbf{6 7}$ suitable for X-ray diffraction analysis are formed after four days. Yield: 0.670 g $\left(88 \%\right.$ ). Mp $176{ }^{\circ} \mathrm{C} .{ }^{1} \mathrm{H}$ NMR $\left(200 \mathrm{MHz}, \mathrm{C}_{6} \mathrm{D}_{6}\right): \delta 8.24$ (d, $\left.2 \mathrm{H}, o-\mathrm{Ph}\right), 7.60(\mathrm{~d}, 1 \mathrm{H}, o-\mathrm{Py}), 7.33(\mathrm{t}, 2 \mathrm{H}, m-\mathrm{Ph})$, 7.03-7.16 (m, 6H, Ar- $H$ ), 6.83-6.79 (m, 2H, $p$-Ph, $p$-Py), 6.3 (m, 2H, $m$-Py), 4.95 (s, $1 \mathrm{H}, \gamma-\mathrm{CH}$ ), 4.21 (sept, $1 \mathrm{H}$, $\left.\mathrm{CH}\left(\mathrm{CH}_{3}\right)_{2}\right), 3.79$ (sept, $\left.1 \mathrm{H}, \mathrm{CH}\left(\mathrm{CH}_{3}\right)_{2}\right), 3.66$ (sept, $\left.1 \mathrm{H}, \mathrm{CH}\left(\mathrm{CH}_{3}\right)_{2}\right), 3.32$ (sept, $\left.1 \mathrm{H}, \mathrm{CH}\left(\mathrm{CH}_{3}\right)_{2}\right), 1.86$ (s, $6 \mathrm{H}$, $\left.\mathrm{N}\left(\mathrm{CH}_{3}\right)_{2}\right), 1.79$ (s, 3H, $\left.\mathrm{CH}_{3}\right), 1.72\left(\mathrm{~s}, 3 \mathrm{H}, \mathrm{CH}_{3}\right), 1.56$ (d, $\left.6 \mathrm{H}, \mathrm{CH}\left(\mathrm{CH}_{3}\right)_{2}\right), 1.31-1.11$ (m, $\left.15 \mathrm{H}, \mathrm{CH}\left(\mathrm{CH}_{3}\right)_{2}\right),-0.12$ (d, $\left.3 \mathrm{H}, \mathrm{CH}\left(\mathrm{CH}_{3}\right)_{2}\right)$ ppm. ${ }^{119} \mathrm{Sn}\left\{{ }^{1} \mathrm{H}\right\}$ NMR $\left(186.50 \mathrm{MHz}, \mathrm{C}_{6} \mathrm{D}_{6}\right): \delta-328$ ppm. EI-MS: $m / z(\%) 537(100)[\mathrm{M}-$ $\left.\mathrm{OCPh}(2-\mathrm{Py}) \mathrm{NMe}_{2}\right]^{+}$. Anal. calcd for $\mathrm{C}_{43} \mathrm{H}_{56} \mathrm{~N}_{4} \mathrm{OSn}$ (764.35): C 67.63, H 7.39, N 7.34; Found C 67.79, H 7.96, N 7.24 .

11.4.66. Synthesis of $\mathrm{LSnOCPh}\left(\mathrm{CF}_{3}\right)\left(\mathrm{NMe}_{2}\right)(\mathbf{6 8})$

A solution of 2,2,2-trifluoro acetophenone $(0.175 \mathrm{~g}, 1.00 \mathrm{mmol}$ in $5 \mathrm{~mL}$ toluene) was added by cannula to a solution of $\mathbf{6 6}(0.54 \mathrm{~g}, 1.00 \mathrm{mmol}$ in toluene $20 \mathrm{~mL})$ at room temperature. After $12 \mathrm{~h}$ all volatiles were removed in vacuo, and the remaining residue was extracted with $n$-hexane $(25 \mathrm{~mL})$. The solvent was removed and compound 68 obtained as a powder. Yield: $0.550 \mathrm{~g}(73 \%)$. Mp $178{ }^{\circ} \mathrm{C} .{ }^{1} \mathrm{H}$ NMR $\left(200 \mathrm{MHz}, \mathrm{C}_{6} \mathrm{D}_{6}\right): \delta 7.32(\mathrm{~d}$, $2 \mathrm{H}, o-\mathrm{Ph}$ ), 7.08-7.17 (m, 6H, Ar-H ), 7.00 (m, 3H, $p$ - $\mathrm{Ph}, m-\mathrm{Ph}$ ), 4.66 (s, $1 \mathrm{H}, \gamma-\mathrm{CH}), 3.99$ (sept, $\left.2 \mathrm{H}, \mathrm{CH}\left(\mathrm{CH}_{3}\right)_{2}\right)$, 3.14 (sept, $\left.2 \mathrm{H}, \mathrm{CH}\left(\mathrm{CH}_{3}\right)_{2}\right), 1.99$ (s, $\left.6 \mathrm{H}, \mathrm{N}\left(\mathrm{CH}_{3}\right)_{2}\right), 1.59$ (s, $\left.3 \mathrm{H}, \mathrm{CH}\left(\mathrm{CH}_{3}\right)_{2}\right), 1.55$ (s, $\left.3 \mathrm{H}, \mathrm{CH}_{3}\right), 1.53\left(\mathrm{~s}, 3 \mathrm{H}, \mathrm{CH}_{3}\right)$, $1.51\left(\mathrm{~d}, 3 \mathrm{H}, \mathrm{CH}\left(\mathrm{CH}_{3}\right)_{2}\right), 1.12-1.27\left(\mathrm{~m}, 15 \mathrm{H}, \mathrm{CH}\left(\mathrm{CH}_{3}\right)_{2}\right), 1.14\left(\mathrm{~d}, 6 \mathrm{H}, \mathrm{CH}\left(\mathrm{CH}_{3}\right)_{2}\right) \mathrm{ppm} .{ }^{119} \mathrm{Sn}\left\{{ }^{1} \mathrm{H}\right\} \mathrm{NMR}$ $\left(186.50 \mathrm{MHz}, \mathrm{C}_{6} \mathrm{D}_{6}\right): \delta-232 \mathrm{ppm}$. EI-MS: $m / z(\%) 537(100)\left[\mathrm{M}-\mathrm{OCPh}\left(\mathrm{CF}_{3}\right) \mathrm{NMe}_{2}\right]^{+}$. Anal. calcd for $\mathrm{C}_{39} \mathrm{H}_{52} \mathrm{~F}_{3} \mathrm{~N}_{3} \mathrm{OSn}$ (755.31): C 62.08, H 6.95, N 5.57; Found C 61.55, H 6.67, N 5.47.

\subsubsection{Synthesis of $\mathrm{LSnCCCO}_{2} \mathrm{Me}(69)$}

A solution of $\mathrm{HCCCO}_{2} \mathrm{Me}(0.085 \mathrm{~g}, 1.00 \mathrm{mmol}$ in $5 \mathrm{~mL}$ toluene $)$ was added drop by drop by cannula to a solution of $\mathbf{6 6}(0.540 \mathrm{~g}, 1.00 \mathrm{mmol}$ in toluene $15 \mathrm{~mL})$ at room temperature. After $0.5 \mathrm{~h}$ under constant stirring at ambient temperature all volatiles were removed in vacuo, and the remaining residue was extracted with $n$ hexane $(15 \mathrm{~mL})$ and concentrated to about $5 \mathrm{~mL}$ and stored in a $-30{ }^{\circ} \mathrm{C}$ freezer. After two days yellow crystalline compound of 69 are formed. Yield: $0.545 \mathrm{~g}(88 \%)$. Mp $124{ }^{\circ} \mathrm{C} .{ }^{1} \mathrm{H}$ NMR ( $\left.300 \mathrm{MHz}, \mathrm{C}_{6} \mathrm{D}_{6}\right): \delta$ 7.03-7.16 (m, $6 \mathrm{H}, \mathrm{Ar}-H$ ), 4.98 (s, $1 \mathrm{H}, \gamma-\mathrm{CH}$ ), 3.88 (sept, $\left.2 \mathrm{H}, \mathrm{CH}\left(\mathrm{CH}_{3}\right)_{2}\right), 3.29$ (s, $\left.3 \mathrm{H}, \mathrm{CO}_{2} \mathrm{CH}_{3}\right), 3.23$ (sept, $2 \mathrm{H}, \mathrm{CH}\left(\mathrm{CH}_{3}\right)_{2}$ ), $1.58\left(\mathrm{~s}, 6 \mathrm{H}, \mathrm{CH}_{3}\right), 1.47\left(\mathrm{~d}, 6 \mathrm{H}, \mathrm{CH}\left(\mathrm{CH}_{3}\right)_{2}\right), 1.36\left(\mathrm{~d}, 6 \mathrm{H}, \mathrm{CH}\left(\mathrm{CH}_{3}\right)_{2}\right), 1.21\left(\mathrm{~d}, 6 \mathrm{H}, \mathrm{CH}\left(\mathrm{CH}_{3}\right)_{2}\right), 1.08(\mathrm{~d}, 6 \mathrm{H}$, $\left.\mathrm{CH}\left(\mathrm{CH}_{3}\right)_{2}\right)$ ppm. ${ }^{119} \mathrm{Sn}\left\{{ }^{1} \mathrm{H}\right\}$ NMR $\left(111.92 \mathrm{MHz}, \mathrm{C}_{6} \mathrm{D}_{6}\right): \delta-254.91$ ppm. EI-MS: $m / z(\%) 620(85)[\mathrm{M}]^{+}, 562$ (100) $[\mathrm{M}-(\mathrm{Me}, i \mathrm{Pr})]^{+}$. Anal. calcd for $\mathrm{C}_{33} \mathrm{H}_{44} \mathrm{~N}_{2} \mathrm{O}_{2} \mathrm{Sn}$ (620.24): C 63.99, H 7.16, N 4.52; Found C 63.28, H 7.51, N 4.73 .

11.4.68. Synthesis of $\mathrm{LSnCCCO}_{2} \mathrm{Et}$ (70)

A solution of $\mathrm{HCCCO}_{2} \mathrm{Et}(0.100 \mathrm{~g}, 1.00 \mathrm{mmol}$ in $5 \mathrm{~mL}$ toluene $)$ was added drop by drop by cannula to a solution of $66(0.540 \mathrm{~g}, 1.00 \mathrm{mmol}$ in toluene $15 \mathrm{~mL})$ at room temperature. After $0.5 \mathrm{~h}$ under constant stirring at ambient temperature the red solution turned deep-red. All volatiles were removed in vacuo, and the remaining 
residue was extracted with $n$-hexane $(15 \mathrm{~mL})$ and concentrated to about $5 \mathrm{~mL}$ and stored in a $-30{ }^{\circ} \mathrm{C}$ freezer. Yellow crystals of 70 suitable for X-ray diffraction analysis are formed after two days. Yield: $0.495 \mathrm{~g}(78 \%)$. Mp $164{ }^{\circ} \mathrm{C} .{ }^{1} \mathrm{H}$ NMR $\left(200 \mathrm{MHz}, \mathrm{C}_{6} \mathrm{D}_{6}\right): \delta$ 7.05-7.19 (m, 6H, Ar- $H$ ), 4.99 (s, $\left.1 \mathrm{H}, \gamma-\mathrm{CH}\right), 3.84-4.01(\mathrm{~m}, 4 \mathrm{H}$, $\left.\mathrm{CH}\left(\mathrm{CH}_{3}\right)_{2}, \mathrm{CH}_{2} \mathrm{CH}_{3}\right), 3.26$ (sept, 2H, $\left.\mathrm{CH}\left(\mathrm{CH}_{3}\right)_{2}\right), 1.60$ (s, 6H, $\left.\mathrm{CH}_{3}\right), 1.51$ (d, 6H, $\left.\mathrm{CH}\left(\mathrm{CH}_{3}\right)_{2}\right), 1.40(\mathrm{~d}, 6 \mathrm{H}$, $\left.\mathrm{CH}\left(\mathrm{CH}_{3}\right)_{2}\right), 1.23\left(\mathrm{~d}, 6 \mathrm{H}, \mathrm{CH}\left(\mathrm{CH}_{3}\right)_{2}\right), 1.11\left(\mathrm{~d}, 6 \mathrm{H}, \mathrm{CH}\left(\mathrm{CH}_{3}\right)_{2}\right), 0.92\left(\mathrm{t}, 3 \mathrm{H}, \mathrm{CH}_{2} \mathrm{CH}_{3}\right)$ ppm. ${ }^{119} \mathrm{Sn} \mathrm{NMR}(186.50$ $\left.\mathrm{MHz}, \mathrm{C}_{6} \mathrm{D}_{6}\right): \delta-252.97$ ppm. EI-MS: $m / z$ (\%) 562 (100) [M-(Et, $\left.\left.i \mathrm{Pr}\right)\right]^{+}$. Anal. calcd for $\mathrm{C}_{34} \mathrm{H}_{46} \mathrm{~N}_{2} \mathrm{O}_{2} \mathrm{Sn}(634.26)$ : C 64.47, H 7.32, N 4.42; Found C 63.88, H 6.98, N 4.48.

11.4.69. Synthesis of $\mathrm{LSnClFe}(\mathrm{CO})_{4}(\mathbf{7 1})$

A flask was charged with $\mathrm{LSnCl}(0.570 \mathrm{~g}, 1.00 \mathrm{mmol})$ and $\mathrm{Fe}_{2}(\mathrm{CO})_{9}(0.370 \mathrm{~g}, 1.00 \mathrm{mmol})$ in $\mathrm{THF}(30 \mathrm{~mL})$. The solution was stirred $24 \mathrm{~h}$ at ambient temperature. The byproduct was removed by filtration over celite, resulting in a clear pale brown filtrate. From the resulting solution the volatiles were removed, giving a pale brown solid of 71. The crystals suitable for X-ray structural analysis were obtained from the saturated toluene solution of 71.Yield: $0.630 \mathrm{~g}(85 \%)$. Mp $230{ }^{\circ} \mathrm{C}$. IR (KBr pellet): $\tilde{v}=2043 \mathrm{~s}, 1968 \mathrm{~s}, 1939 \mathrm{~s}(\mathrm{CO}) \mathrm{cm}^{-1} .{ }^{1} \mathrm{H}$ NMR $(200$ $\left.\mathrm{MHz}, \mathrm{C}_{6} \mathrm{D}_{6}, 25^{\circ} \mathrm{C}\right): \delta 7.03-7.16(\mathrm{~m}, 6 \mathrm{H}, \mathrm{ArH}), 5.09(\mathrm{~s}, 1 \mathrm{H}, \gamma-\mathrm{CH}), 3.79$ (sept, 2H, $\left.\mathrm{CH}\left(\mathrm{CH}_{3}\right)_{2}\right), 3.06$ (sept, $2 \mathrm{H}$, $\left.\mathrm{CH}\left(\mathrm{CH}_{3}\right)_{2}\right), 1.52\left(\mathrm{~s}, 6 \mathrm{H}, \mathrm{CH}_{3}\right), 1.49\left(\mathrm{~d}, 6 \mathrm{H}, \mathrm{CH}\left(\mathrm{CH}_{3}\right)_{2}\right), 1.35\left(\mathrm{~d}, 6 \mathrm{H}, \mathrm{CH}\left(\mathrm{CH}_{3}\right)_{2}\right), 1.17\left(\mathrm{~d}, 6 \mathrm{H}, \mathrm{CH}\left(\mathrm{CH}_{3}\right)_{2}\right), 1.01$ $\left(\mathrm{d}, 6 \mathrm{H}, \mathrm{CH}\left(\mathrm{CH}_{3}\right)_{2}\right)$ ppm. ${ }^{119} \mathrm{Sn} \mathrm{NMR}\left(111.92 \mathrm{MHz}, \mathrm{C}_{6} \mathrm{D}_{6}, 25{ }^{\circ} \mathrm{C}\right): \delta 88.98 \mathrm{ppm}$. EI-MS $(70 \mathrm{eV} ; \mathrm{m} / z(\%)): 628$ (100) $[\mathrm{M}-4 \mathrm{CO}]^{+}$. Anal. Calcd for $\mathrm{C}_{33} \mathrm{H}_{41} \mathrm{ClFeN}_{2} \mathrm{O}_{4} \mathrm{Sn}$ (740.11): C 53.58, H 5.59, N 3.79; Found: C 53.29, $\mathrm{H}$ $5.95, \mathrm{~N} 3.66$.

11.4.70. Synthesis of $\mathrm{LSn}\left(\mathrm{NMe}_{2}\right) \mathrm{Fe}(\mathrm{CO})_{4}$ (72)

A flask was charged with 66 (5.80 g, $10.00 \mathrm{mmol})$ and $\mathrm{Fe}_{2}(\mathrm{CO})_{9}(3.70 \mathrm{~g}, 10.20 \mathrm{mmol})$ in THF (140 mL). The solution was stirred $24 \mathrm{~h}$ at ambient temperature. The byproduct was removed by filtration over celite, resulting in a clear pale brown filtrate. From the resulting solution the volatiles were removed, giving a pale brown solid. Crystallization of the crude product was attained by saturated THF solution of 72 and keeping it at $-30{ }^{\circ} \mathrm{C}$ temperature. 72 deposited as pale orange crystals as a formula $\mathbf{7 2}_{2} \cdot \mathbf{T H F}$. Yield: $6.35 \mathrm{~g}(85 \%)$. Mp $218{ }^{\circ} \mathrm{C}$. IR (KBr pellet): $\tilde{v}=2034 \mathrm{~s}, 1957 \mathrm{~s}, 1935 \mathrm{~s}(\mathrm{CO}) \mathrm{cm}^{-1} .{ }^{1} \mathrm{H} \mathrm{NMR}\left(300.13 \mathrm{MHz}, \mathrm{C}_{6} \mathrm{D}_{6}, 25^{\circ} \mathrm{C}\right): \delta 7.07-7.16(\mathrm{~m}, 6 \mathrm{H}$, $\mathrm{ArH}), 4.75$ (s, 1H, $\gamma-\mathrm{CH}), 3.27$ (sept, 2H, $\left.\mathrm{CH}\left(\mathrm{CH}_{3}\right)_{2}\right), 3.15$ (sept, 2H, $\left.\mathrm{CH}\left(\mathrm{CH}_{3}\right)_{2}\right), 1.49$ (s, 6H, $\left.\mathrm{CH}_{3}\right), 1.42(\mathrm{~d}, 6 \mathrm{H}$, $\left.\mathrm{CH}\left(\mathrm{CH}_{3}\right)_{2}\right), 1.32\left(\mathrm{~d}, 6 \mathrm{H}, \mathrm{CH}\left(\mathrm{CH}_{3}\right)_{2}\right), 1.21\left(\mathrm{~d}, 6 \mathrm{H}, \mathrm{CH}\left(\mathrm{CH}_{3}\right)_{2}\right), 1.01\left(\mathrm{~d}, 6 \mathrm{H}, \mathrm{CH}\left(\mathrm{CH}_{3}\right)_{2}\right)$ ppm. ${ }^{119} \mathrm{Sn} \mathrm{NMR}(111.92$ $\left.\mathrm{MHz}, \mathrm{C}_{6} \mathrm{D}_{6}, 25^{\circ} \mathrm{C}\right): \delta 94.00$ ppm. EI-MS (70 eV; $\left.m / z(\%)\right): 749$ (45) [M] $]^{+}, 705$ (100) [M-NMe $]^{+}$. Anal. Calcd for $\mathrm{C}_{35} \mathrm{H}_{47} \mathrm{FeN}_{3} \mathrm{O}_{4} \mathrm{Sn}$ (749.19): C 56.18, H 6.33, N 5.62; Found: C 56.24, H 6.78, N 5.51.

11.4.71. Synthesis of $\mathrm{LSn}(\mathrm{OH}) \mathrm{Fe}(\mathrm{CO})_{4}$ (73)

Degassed water $(72 \mu \mathrm{L}, 4.00 \mathrm{mmol})$ was added to a solution of $72(3.00 \mathrm{~g}, 4.00 \mathrm{mmol})$ in THF (50 mL) at -60 ${ }^{\circ} \mathrm{C}$. Then, it was taken to room temperature and stirred for $1 \mathrm{~h}$. Followed by removal of the solvent in a vacuum to give compound $\mathbf{7 3}$ as a solid. It was washed with a small amount of $n$-hexane and crystallized from toluene at $-32{ }^{\circ} \mathrm{C}$ to exhibit compound 73 as brown crystals. Yield: $2.10 \mathrm{~g}(73 \%)$. Mp $233{ }^{\circ} \mathrm{C}$. IR (KBr pellet): $\tilde{v}=3615$ $\mathrm{s}(\mathrm{OH}), 2038 \mathrm{~s}, 1959 \mathrm{~s}, 1944 \mathrm{~s}(\mathrm{CO}) \mathrm{cm}^{-1} .{ }^{1} \mathrm{H}$ NMR $\left(300.13 \mathrm{MHz}, \mathrm{C}_{6} \mathrm{D}_{6}, 25^{\circ} \mathrm{C}\right): \delta 7.04-7.15(\mathrm{~m}, 6 \mathrm{H}, \mathrm{ArH}), 4.86$ (s, 1H, $\gamma$-CH), 3.75 (sept, 2H, CH( $\left.\left(\mathrm{CH}_{3}\right)_{2}\right), 3.07$ (sept, 2H, $\left.\mathrm{CH}\left(\mathrm{CH}_{3}\right)_{2}\right), 1.66$ (s, 1H, OH), $1.51\left(\mathrm{~s}, 6 \mathrm{H}, \mathrm{CH}_{3}\right), 1.39$ $\left(\mathrm{d}, 6 \mathrm{H}, \mathrm{CH}\left(\mathrm{CH}_{3}\right)_{2}\right), 1.34\left(\mathrm{~d}, 6 \mathrm{H}, \mathrm{CH}\left(\mathrm{CH}_{3}\right)_{2}\right), 1.18\left(\mathrm{~d}, 6 \mathrm{H}, \mathrm{CH}\left(\mathrm{CH}_{3}\right)_{2}\right), 1.04\left(\mathrm{~d}, 6 \mathrm{H}, \mathrm{CH}\left(\mathrm{CH}_{3}\right)_{2}\right) \mathrm{ppm} .{ }^{119} \mathrm{Sn} \mathrm{NMR}$ (111.92 MHz, $\left.\mathrm{C}_{6} \mathrm{D}_{6}, 25^{\circ} \mathrm{C}\right): \delta 45.03$ ppm. EI-MS (70 eV; $\left.m / z(\%)\right): 722(10)[\mathrm{M}]^{+}, 705(100)[\mathrm{M}-\mathrm{OH}]^{+}, 666$ (85) $\left[\mathrm{M}-2 \mathrm{CO}^{+}\right.$. Anal. Calcd for $\mathrm{C}_{33} \mathrm{H}_{42} \mathrm{FeN}_{2} \mathrm{O}_{5} \mathrm{Sn}$ (722.15): C 54.95, H 5.87, N 3.88; Found: C 55.61, H 6.43, N 4.17 .

\subsection{Crystallographic Details}

Complete crystallographic data are deposited at the Cambridge Crystallographic Data Centre, where it can be downloaded free of charge from www.ccdc.cam.ac.uk/ data_request/cif: at CCDC-697821 (1), CCDC-743152 (2), CCDC-743153 (3), CCDC-706811 (4), CCDC-706621 (6), CCDC-730408 (7), CCDC-738339 (8), CCDC706622 (10), CCDC-706623 (11), CCDC-730403 (12), CCDC-706624 (13), CCDC-710236 (14), CCDC667925 (16), CCDC-713790 (17), CCDC-715866 (18), CCDC-709476 (21), CCDC-709478 (22), CCDC709477 (24), CCDC-730399 (26), CCDC-730401 (27), CCDC-709479 (29), CCDC-730400 (31), CCDC- 
705305 (36), CCDC-745457 (40), CCDC-705305 (42), CCDC-745454 (43), CCDC-715867 (44), CCDC715868 (45), CCDC-715869 (46), CCDC-741341 (47), CCDC-720891 (48), CCDC-717497 (51), CCDC740333 (52), CCDC-740334 (54), CCDC-713788 (55), CCDC-713789 (56), CCDC-734212 (58), CCDC728257 (59), CCDC-745456 (60), CCDC-730406 (61), CCDC-697820 (64), CCDC-706812 (65), CCDC743154 (67), CCDC-743155 (70), CCDC-740971 (72), CCDC-740972 (73). 


\section{References}

[1] L. Bourget-Merle, M. F. Lappert, J. R. Severn, Chem. Rev. 2002, 102, 3031-3065.

[2] P. L. Holland, Acc. Chem. Res. 2008, 41, 905-914.

[3] D. J. Mindiola, Acc. Chem. Res. 2006, 39, 813-821.

[4] C. J. Cramer, W. B. Tolman, Acc. Chem. Res. 2007, 40, 601-608.

[5] H. W. Roesky, S. Singh, V. Jancik, V. Chandrasekhar, Acc. Chem. Res. 2004, 37, 969-981.

[6] W. E. Piers, D. J. H. Emslie, Coord. Chem. Rev. 2002, 233-234, 131-155.

[7] M. Rahim, N. J. Taylor, S. Xin, S. Collins, Organometallics 1998, 17, 1315-1323.

[8] S. G. McGeachin, Can. J. Chem. 1968, 46, 1903-1912.

[9] J. E. Parks, R. H. Holm, Inorg. Chem. 1968, 7, 1408-1416.

[10] S. Nagendran, H. W. Roesky, Organometallics 2008, 27, 457-492.

[11] (a) S. Pfirrmann, C. Limberg, C. Herwig, R. Stößer, B. Ziemer, Angew. Chem. 2009, 121, 3407-3411; Angew. Chem. Int. Ed. 2009, 48, 3357-3361. (b) K. Ding, A. W. Pierpont, W. W. Brennessel, G. Lukat-Rodgers, K. R. Rodgers, T. R. Cundari, E. Bill, P. L. Holland, J. Am. Chem. Soc. 2009, 131, 9471-9472.

[12] J. Feldman, S. J. McLain, A. Parthasarathy, W. J. Marshall, J. C. Calabrese, S. D. Arthur, Organometallics 1997, 16, 1514-1516.

[13] C. Cui, H. W. Roesky, H.-G. Schmidt, M. Noltemeyer, H. Hao, F. Cimpoesu, Angew. Chem. 2000, 112, 4444-4446; Angew. Chem. Int. Ed. 2000, 39, 4274-4276.

[14] S. P. Green, C. Jones, A. Stasch, Science 2007, 318, 1754-1757.

[15] F. Basuli, B. C. Bailey, J. C. Huffman, D. J. Mindiola, Organometallics 2005, 24, 3321-3334.

[16] P. G. Hayes, W. E. Piers, M. Parvez, J. Am. Chem. Soc. 2003, 125, 5622-5623.

[17] U. Fekl, W. Kaminsky, K. I. Goldberg, J. Am. Chem. Soc. 2001, 123, 6423-6424.

[18] N. A. Eckert, S. Vaddadi, S. Stoian, R. J. Lachicotte, T. R. Cundari, P. L. Holland, Angew. Chem. 2006, 118, 7022-7025; Angew. Chem. Int. Ed. 2006, 45, 6868-6871.

[19] N. C. Tomson, A. Yan, J. Arnold, R. G. Bergman, J. Am. Chem. Soc. 2008, 130, $11262-$ 11263.

[20] N. Kuhn, A. Kuhn, R. Boese, N. Augart, J. Chem. Soc. Chem. Commun. 1989, 975976.

[21] N. Kuhn, A. Kuhn, M. Speis, D. Bläser, R. Boese, Chem. Ber. 1990, 123, 1301-1306.

[22] D. J. E. Spencer, A. M. Reynolds, P. L. Holland, B. A. Jazdzewski, C. Duboc-Toia, L. L. Pape, S. Yokota, Y. Tachi, S. Itoh, W. B. Tolman, Inorg. Chem. 2002, 41, 63076321.

[23] C. E. Radzewich, M. P. Coles, R. F. Jordan, J. Am. Chem. Soc. 1998, 120, 9384-9385.

[24] P. B. Hitchcock, M. F. Lappert, J. E. Nycz, Chem. Commun. 2003, 1142-1143.

[25] P. J. Ragogna, N. Burford, M. D'eon, R. McDonald, Chem. Commun. 2003, 1052-1053.

[26] D. T. Carey, E. K. Cope-Eatough, E. Vilaplana- Mafé, F. S. Mair, R. G. Pritchard, J. E. Warren, R. J. Woods, Dalton Trans. 2003, 1083-1093.

[27] E. A. Gregory, R. J. Lachicotte, P. L. Holland, Organometallics 2005, 24, 1803-1805.

[28] Y. Ding, H. Hao, H. W. Roesky, M. Noltemeyer, H.-G. Schmidt, Organometallics 2001, 20, 48064811.

[29] Y. Ding, Q. Ma, H. W. Roesky, R. Herbst-Irmer, I. Usón, M. Noltemeyer, H.-G. Schmidt, Organometallics 2002, 21, 5216-5220.

[30] H. Zhu, J. Chai, A. Stasch, H. W. Roesky, T. Blunck, D. Vidovic, J. Magull, H.-G. Schmidt, M. Noltemeyer, Eur. J. Inorg. Chem. 2004, 4046-4051.

[31] F. Basuli, J. C. Huffman, D. J. Mindiola, Inorg. Chem. 2003, 42, 8003-8010.

[32] B. Qian, S. W. Baek, M. R. Smith, Polyhedron 1999, 18, 2405-2414.

[33] S. Harder, Angew. Chem. 2003, 115, 3553-3556; Angew. Chem. Int. Ed. 2003, 42, 3430-3434.

[34] D. Adhikari, F. Basuli, J. H. Orlando, X. Gao, J. C. Huffman, M. Pink, D. J. Mindiola, Organometallics 2009, 28, 4115-4125.

[35] P. H. M. Budzelaar, A. B. van Oort, A. G. Orpen, Eur. J. Inorg. Chem. 1998, 1485-1494. 
[36] M. Driess, S. Yao, M. Brym, C.van Wüllen, D. Lentz, J. Am. Chem. Soc. 2006, 128, 9628-9629.

[37] M. Driess, S. Yao, M. Brym, C. van Wüllen, Angew. Chem. 2006, 118, 4455-4458; Angew. Chem. Int. Ed. 2006, 45, 4349-4352.

[38] P. Jutzi, H. J. Hoffmann, D. J. Brauer, C. Krüger, Angew. Chem. 1973, 85, 1116-1117; Angew. Chem. Int. Ed. Engl. 1973, 12, 1002-1003.

[39] F. X. Kohl, P. Jutzi, J. Organomet. Chem. 1987, 329, C17-C20.

[40] L. M. Engelhardt, B. S. Jolly, M. F. Lappert, C. L. Raston, A. L. White, J. Chem. Soc. Chem. Commun. 1988, 336-338.

[41] C.-W. So, H. W. Roesky, J. Mugull, R. B. Oswald, Angew. Chem. 2006, 118, 4052-4054; Angew. Chem. Int. Ed. 2006, 45, 3948-3950.

[42] R. S. Ghadwal, H. W. Roesky, S. Merkel, J. Henn, D. Stalke, Angew. Chem. 2009, 121, 5793-5796; Angew. Chem. Int. Ed. 2009, 48, 5683-5686.

[43] A. C. Filippou, O. Chernov, Gregor. Schnakenburg, Angew. Chem. 2009, 121, 5797-5800; Angew. Chem. Int. Ed. 2009, 48, 5687-5690.

[44] E. Rivard, P. P. Power, Inorg. Chem. 2007, 46, 10047-10064.

[45] E. F. Murphy, R. Murugavel, H. W. Roesky, Chem. Rev. 1997, 97, 3425-3468.

[46] H. W. Roesky, I. Haiduc, J. Chem. Soc. Dalton Trans. 1999, 2249-2264.

[47] H. W. Roesky, Inorg. Chem. 1999, 38, 5934-5943.

[48] D. J. Brauer, J. Wilke, R. Eujen, J. Organomet. Chem. 1986, 316, 261-269.

[49] E. Lukevics, S. Belyakov, P. Arsenyan, J. Popelis, J. Organomet. Chem. 1997, 549, 163-1654.

[50] D. J. Brauer, H. Bürger, R. Eujen, Angew. Chem. 1980, 92, 859-860; Angew. Chem. Int. Ed. Engl. 1980, 19, 836-837.

[51] R. W. Chorley, D. Ellis, P. B. Hitchcock, M. F. Lappert, Bull. Soc. Chim. Fr. 1992, 129, $599-604$.

[52] (a) P. Rivière, J. Satgé, A. Castel, H. Normant, C. R. Acad. sci. Paris. 1976, 282, 971. (b) P. Rivière, J. Satgé, A. Castel, H. Normant, C. R. Acad. Sci. Paris. 1977, 284, 395. (c) A. Castel, P. Rivière, J. Satgé, A. Cazes, H. Normant, C. R. Acad. Sci. Paris. 1978, 287, 205.

[53] F. A. Cotton, G. Wilkinson, C. A. Murillo, M. Bochmann, Advanced Inorganic Chemistry, 6 th ed. Wiley-Interscience Publication, New York, 1999.

[54] R. C. McDonald, H. H. Hau, K. Eriks, Inorg. Chem. 1976, 15, 762-765.

[55] M. Mehring, I. Vrasidas, D. Horn, M. Schürmann, K. Jurkschat, Organometallics 2001, 20, 4647-4653.

[56] W.-P. Leung, W.-H. Kwok, Z.-Y. Zhou, T. C. W, Mak, Organometallics 2003, 22, 1751-1755.

[57] P. Švec, P. Novák, M. Nádvorník, Z. Padělková, I. Císařová, L. Kolářová, A. Růžička, J. Holeček, J. Fluorine Chem. 2007, 128, 1390-1395.

[58] R. A. Varga, K. Jurkschat, C. Silvestru, Eur. J. Inorg. Chem. 2008, 708-716.

[59] H. D. Kaesz, R. B. Saillant, Chem. Rev. 1972, 72, 231-281.

[60] G. S. McGrady, G. Guilera, Chem. Soc. Rev. 2003, 32, 383-392.

[61] S. Aldridge, A. J. Downs, Chem. Rev. 2001, 101, 3305-3365.

[62] H.-J. Himmel, Dalton. Trans. 2003, 3639-3649.

[63] H.-J. Himmel, Z. Anorg. Allg. Chem. 2005, 631, 1551-1564.

[64] N. W. Mitzel, Angew. Chem. 2003, 115, 3984-3986; Angew. Chem. Int. Ed. 2003, 42, 3856-3858.

[65] W. M. Mueller, J. P. Blackledge, G. G. Libowitz, Metal Hydrides, Academic Press, London, 1968, pp. $1-21$.

[66] F. Lefebvre, J.-M. Basset, Main Group Met. Chem. 2002, 25, 15-32.

[67] J. Zhao, A. S. Goldman, J. F. Hartwig, Science 2005, 307, 1080-1082.

[68] R. F. Service, Science 2004, 305, 958-961.

[69] J. A. Turner, Science 2004, 305, 972-974.

[70] W. Grochala, P. P. Edwards, Chem. Rev. 2004, 104, 1283-1315.

[71] F. Schüth, B. Bogdanović, M. Felderhoff, Chem. Commun. 2004, 2249-2258.

[72] Y. Yu, A. R. Sadique, J. M. Smith, T. R. Dugan, R. E. Cowley, W. W. Brennessel, C. J. Flaschenriem, E. Bill, T. R. Cundari, P. L. Holland, J. Am. Chem. Soc. 2008, 130, 6624-6638.

[73] M. Arrowsmith, M. S. Hill, D. J. MacDougall, M. F. Mahon, Angew. Chem. 2009, 121, 4073-4076; Angew. Chem. Int. Ed. 2009, 48, 4013-4016. 
[74] R. West, J. Organomet. Chem. 1986, 300, 327-346.

[75] R. D. Miller, J. Michl, Chem. Rev. 1989, 89, 1359-1410.

[76] M. Birot, J.-P. Pillot, J. Dunoguès, Chem. Rev. 1995, 95, 1443-1477.

[77] J. Kouvetakis, A. Haaland, D. J. Shorokhov, H. V. Volden, G. V. Girichev, V. I. Sokolov, P. Matsunaga, J. Am. Chem. Soc. 1998, 120, 6738-6774.

[78] P. Braunstein, X. Morise, Chem. Rev. 2000, 100, 3541-3552.

[79] L. W. Pineda, V. Jancik, K. Starke, R. B. Oswald, H. W. Roesky, Angew. Chem. 2006, 118, 2664-2667; Angew. Chem. Int. Ed. 2006, 45, 2602-2605.

[80] B. E. Eichler, P. P. Power, J. Am. Chem. Soc. 2000, 122, 8785-8786.

[81] A. F. Richards, A. D. Phillips, M. M. Olmstead, P. P. Power, J. Am. Chem. Soc. 2003, 125, 3204-3205.

[82] E. Rivard, R. C. Fischer, R. Wolf, Y. Peng, W. A. Merrill, N. D. Schley, Z. Zhu, L. Pu, J. C. Fettinger, S. J. Teat, I. Nowik, R. H. Herber, N. Takagi, S. Nagase, P. P. Power, J. Am. Chem. Soc. 2007, 129, 16197-16208.

[83] G. Trinquier, J. Am. Chem. Soc. 1990, 112, 2130-2137.

[84] X. Wang, L. Andrews, G. V. Chertihin, P. F. Souter, J. Phys. Chem. A 2002, 106, 6302-6308.

[85] R. D. Shannon, Acta Cryst. 1976, A 32, 751-767

[86] (a) D. Bourissou, O. Guerret, F. P. Gabbaï, G. Bertrand, Chem. Rev. 2000, 100, 39-91. (b) A. J. Arduengo, III Acc. Chem. Res. 1999, 32, 913-921. (c) D. Enders, O. Niemeier, A. Henseler, Chem. Rev. 2007, 107, 5606-5655.

[87] J. Satgé, M. Massol, P. Rivière, J. Organomet. Chem. 1973, 56, 1-39.

[88] E. J. Bulten, J. G. Noltes, Tetrahedron Lett. 1967, 8, 1443-1447.

[89] P. Jutzi, S. Keitemeyer, B. Neumann, H.-G. Stammler, Organometallics 1999, 18, 4778-4784.

[90] W. P. Neumann, Chem. Rev. 1991, 91, 311-334.

[91] M. Driess, H. Grützmacher, Angew. Chem. 1996, 108, 900-929; Angew. Chem. Int. Ed. Engl. 1996, 35, 828-856.

[92] M. Weidenbruch, Eur. J. Inorg. Chem. 1999, 373-381.

[93] N. Tokitoh, R. Okazaki, Coord. Chem. Rev. 2000, 210, 251-277.

[94] S. E. Boganov, M. V. Egorov, V. I. Faustov, O. M. Nefedov, In The Chemistry of Organic Germanium, Tin and Lead Compounds; Rappoport, Z. Ed.; John Wiley and Sons: New York, 2002, 2, 749-839.

[95] B. E. Eichler, P. P. Power, Inorg. Chem. 2000, 39, 5444-5449.

[96] P. Bleckmann, H. Maly, R. Minkwitz, W. P. Neumann, B. Watta, Tetrahedron Letters 1982, 23, 46554658.

[97] B. Watta, W. P. Neumann, J. Sauer, Organometallics 1985, 4, 1954-1957.

[98] R. Becerra, P. P. Gaspar, C. R. Harrington, W. J. Leigh, I. Vargas-Baca, R. Walsh, D. Zhou, J. Am. Chem. Soc. 2005, 127, 17469-17478.

[99] R. Waterman, P. G. Hayes, T. D. Tilley, Acc. Chem. Res. 2007, 40, 712-719.

[100] M. Denk, R. Lennon, R. Hayashi, R. West, A. Haaland, H. Belyakov, P. Verne, M. Wagner, N. Metzler, J. Am. Chem. Soc. 1994, 116, 2691-2692.

[101] B. Gehrhus, M. F. Lappert, J. Heinicke, R. Boese, D. Blaser, J. Chem. Soc. Chem. Commun. 1995, 1931-1932.

[102] M. Kira, S. Ishida, T. Iwamoto, C. Kabuto, J. Am. Chem. Soc. 1999, 121, 9722-9723.

[103] C.W. So, H. W. Roesky, P. M. Gurubasavaraj, R. B. Oswald, M. T. Gamer, P. G. Jones, S. Blaurock, J. Am. Chem. Soc. 2007, 129, 12049-12054.

[104] H. H. Karsch, U. Keller, S. Gamper, G. Müller, Angew. Chem. 1990, 102, 297-298; Angew. Chem. Int. Ed. Engl. 1990, 29, 295-296.

[105] D. H. Harris, M. F. Lappert, J. B. Pedley, G. J. Sharp, J. Chem. Soc. Dalton Trans. 1976, 945-950.

[106] J. Barrau, J. Escudié, J. Satgé, Chem. Rev. 1990, 90, 283-319.

[107] "Recent Advances in structural chemistry of organic germanium, tin and lead compounds": K. W. Klinkhammer in The Chemistry of Organic Germanium, Tin and Lead Compounds, Vol. 2 (Ed.: Z. Rappoport), Wiley, New York, 2002, chap. 4, pp. 284-332.

[108] O. Kühl, Coord. Chem. Rev. 2004, 248, 411-427.

[109] M. Driess, N. Dona, K. Merz, Dalton Trans. 2004, 3176-3177. 
[110] A. Castel, P. Riviere, J. Satge, M. Ahbala, J. Organomet. Chem. 1987, 331, 11-21.

[111] J. E. Bender IV, A. J. Shusterman, M. M. B. Holl, J. W. Kampf, Organometallics 1999, 18, 1547-1552.

[112] J. Barrau, G. Rima, T. El Amraoui, J. Organomet. Chem. 1998, 570, 163-174.

[113] M. Knorr, E. Hallauer, V. Huch, M. Veith, P. Braunstein, Organometallics 1996, 15, 3868-3875.

[114] W. A. Herrmann, H. J. Kneuper, E. Herdtweck, Chem. Ber. 1989, 122, 433-436.

[115] J. Janssen, J. Magull, H.W. Roesky, Angew. Chem. 2002, 114, 1425-1427; Angew. Chem. Int. Ed. 2002, 41, 1365-1367.

[116] G. Bai, H. W. Roesky, J. Li, T. Labahn, F. Cimpoesu, J. Magull, Organometallics 2003, 22 , 3034-3038.

[117] P. M. Gurubasavaraj, H. W. Roesky, P. M. V. Sharma, R. B. Oswald, V. Dolle, R. Herbst-Irmer, A. Pal, Organometallics 2007, 26, 3346-3351.

[118] B. Nekoueishahraki, A. Jana, H. W. Roesky, D. Stern, D. Stalke, Organometallics 2009, 28, DOI: 10.1021/om900623w

[119] G. Bai, Y. Peng, H.W. Roesky, J. Li, H.-G. Schmidt, M. Noltemeyer, Angew. Chem. 2003, 115, 11641167; Angew. Chem. Int. Ed. 2003, 42, 1132-1135.

[120] G. Bai, S. Singh, H. W. Roesky, M. Noltemeyer, H.-G. Schmidt, J. Am. Chem. Soc. 2005, 127, 34493455.

[121] Y. Yang, T. Schulz, M. John, Z. Yang, V. M. Jiménez-Pérez, H. W. Roesky, P. M. Gurubasavaraj, D. Stalke, H. Ye, Organometallics 2008, 27, 769-777.

[122] Y. Yang, P. M. Gurubasavaraj, H. Ye, Z. Zhang, H. W. Roesky, P. G. Jones, J. Organomet. Chem. 2008, 693, 1455-1461.

[123] V. Jancik, L. W. Pineda, A. C. Stückl, H. W. Roesky, R. Herbst-Irmer, Organometallics 2005, 24, 1511-1515.

[124] S. Singh, V. Jancik, H. W. Roesky, R. Herbst-Irmer, Inorg. Chem. 2006, 45, 949-951.

[125] C. Ruspic, S. Nembenna, A. Hofmeister, J. Magull, S. Harder, H. W. Roesky, J. Am. Chem. Soc. 2006, $128,15000-15004$.

[126] S. P. Sarish, S. Nembenna, S. Nagendran, H. W. Roesky, A. Pal, R. Herbst-Irmer, A. Ringe, J. Magull, Inorg. Chem. 2008, 47, 5971-5977.

[127] L. W. Pineda, V. Jancik, H. W. Roesky, D. Neculai, A. M. Neculai, Angew. Chem. 2004, 116, 14431445; Angew. Chem. Int. Ed. 2004, 43, 1419-1421.

[128] H.W. Roesky, M. G. Walawalkar, R. Murugavel, Acc. Chem. Res. 2001, 34, 201-211.

[129] S. Singh, H. W. Roesky, Dalton Trans. 2007, 1360-1370.

[130] P. M. Gurubasavaraj, S. K. Mandal, H. W. Roesky, R. B. Oswald, A. Pal, M. Noltemeyer, Inorg. Chem. 2007, 46, 1056-1061.

[131] A. G. Davies, P. J. Smith, Comprehensive Organometallic Chemistry (Eds.: G. Wilkinson, F. G. A. Stone, E. W. Abel), Pergamon, Oxford, 1982, pp. 573-583.

[132] G. H. Spikes, Y. Peng, J. C. Fettinger, J. Steiner, P. P. Power, Chem. Commun. 2005, 6041-6043.

[133] D. H. Harris, M. F. Lappert, Chem. Commun. 1974, 895-896.

[134] C. D. Schaeffer, J. J. Zuckerman, J. Am. Chem. Soc. 1974, 96, 7160-7162.

[135] M. J. S. Gynane, D. H. Harris, M. F. Lappert, P. P. Power, P. Riviere, M. Riviere-Baudet, J. Chem. Soc. Dalton Trans. 1977, 2004-2009.

[136] R. W. Chorley, P. B. Hitchcock, M. F. Lappert, W.-P, Leung, P. P. Power, M. M. Olmstead, Inorg. Chim. Acta 1992, 198-200, 203-209.

[137] C. Stanciu, S. S. Hino, M. Stender, A. F. Richards, M. M. Olmstead, P. P. Power, Inorg. Chem. 2005, 44, 2774-2780.

[138] R. Siefken, M. Teichert, D. Chakraborty, H. W. Roesky, Organometallics 1999, 18, 2321-2325.

[139] K. Wraage, H.-G. Schmidt, M. Noltemeyer, H. W. Roesky, Eur. J. Inorg. Chem. 1999, 863.

[140] K. Ruhlandt-Senge, R. A. Bartlett, M. M. Olmstead, P. P. Power, Angew. Chem. 1993, 105, 459-461; Angew. Chem. Int. Ed. Engl. 1993, 32, 425-427.

[141] C. Ackerhans, P. Böttcher, P. Müller, H. W. Roesky, I. Usón, H.-G. Schmidt, M. Noltemeyer, Inorg. Chem. 2001, 40, 3766-3773. 
[142] K. Wraage, A. Kunzel, M. Noltemeyer, H.-G. Schmidt, H. W. Roesky, Angew. Chem. 1995, 107, 29542956; Angew. Chem. Int. Ed. Engl. 1995, 34, 2645-2647.

[143] K. Wraage, L. Lameyer, D. Stalke, H. W. Roesky, Angew. Chem. 1999, 111, 542-543; Angew. Chem. Int. Ed. 1999, 38, 522-523.

[144] M. Rivière-Baudet, A. Morère, J. F. Britten, M. Onyszchuk, J. Organomet. Chem. 1992, 423, C5-C8.

[145] V. Jancik, L. W. Pineda, J. Pinkas, H. W. Roesky, D. Neculai, A. M. Neculai, R. Herbst-Irmer, Angew. Chem. 2004, 116, 2194-2197; Angew. Chem. Int. Ed. 2004, 43, 2142-2145.

[146] Y. Ding, H. W. Roesky, M. Noltemeyer, H.-G. Schmidt, Organometallics 2001, 20, 1190-1194.

[147] (a) H. W. Roesky, K. Keller, J. Fluorine Chem. 1998, 89, 3-4. (b) J. Vela, J. M. Smith, Y. Yu, N. A. Ketterer, C. J. Flaschenriem, R. J. Lachicotte, P. L. Holland, J. Am. Chem. Soc. 2005, 127, 7857-7870.

[148] D. W. Hawker, P. R. Wells, J. Organomet. Chem.1984, 266, 37-44.

[149] S. Thoonen, B.-J. Deelman, G. V. Koten, Chem. Commun. 2001, 1840-1841.

[150] L. Pu, M. M. Olmstead, P. P. Power, Organometallics 1998, 17, 5602-5606.

[151] J. Spielmann, S. Harder, Chem. Eur. J. 2007, 13, 8928-8938.

[152] M. V. Butovskii, O. L. Tok, F. R. Wagner, R. Kempe, Angew. Chem. 2008, 120, 6569-6572; Angew. Chem. Int. Ed. 2008, 47, 6469-6472.

[153] (a) S. Sabo-Etienne, B. Chaudret, Chem. Rev. 1998, 98, 2077-2092. (b) A. J. Hoskin, D. W. Stephan, Coord. Chem. Rev. 2002, 233-234, 107-129. (c) M. Ephritikhine, Chem. Rev. 1997, 97, 2193-2242. (d) Z. Hou, M. Nishiura, T. Shima, Eur. J. Inorg. Chem. 2007, 2535-2545.

[154] (a) T. Hiyama, T. Kusumoto, Comprehensive Organic Synthesis Pergamon: Oxford, U.K. 1991, 8, 763792. (b) C. D. Beard, J. C. Craig, J. Am. Chem. Soc. 1974, 96, 7950-7954. (c) T. Saegusa, Y. Ito, S. Kobayashi, K. Hirota, J. Am. Chem. Soc. 1967, 89, 2240-2241. (d) J. A. Connor, P. D. Rose, R. M. Turner, J. Organomet. Chem. 1973, 55, 111-119. (e) G. A. Razuvaev, J. Organomet. Chem. 1980, 200, 243-259. (f) G. Manuel, G. Bertrand, P. Mazerolles, J. Organomet. Chem. 1978, 146, 7-16. (g) G. F. Bradley, S. R. Stobart, J. Chem. Soc. Dalton Trans. 1974, 264-269. (h) R. D. Adams, F. A. Cotton, W. R. Cullen, D. L. Hunter, L. Mihichuk, Inorg. Chem. 1975, 14, 1395-1399. (i) U. Blaukat, W. P. Neumann, J. Organomet. Chem. 1973, 63, 27-39.

[155] (a) R. A. Kovar, J. O. Callaway, Inorg. Synth. 1976, 22, 37-47. (b) H.W. Roesky, Aldrichimica Acta 2004, 37, 103-108. (c) S. S. Kumar, H.W. Roesky, Dalton Trans. 2004, 3927-3937.

[156] (a) D. J. Darensbourg, R. A. Kudaroski, Adv. Organomet. Chem. 1983, 22, 129-168. (b) P. G. Jessop, T. Ikariya, R. Noyori, Chem. Rev. 1995, 95, 259-272. (c) C. S. Yi, N. Liu, Organometallics 1995, 14, 2616-2617. (d) D. G. Gibson, Chem. Rev. 1996, 96, 2063-2095. (e) M. A. McLoughlin, N. L. Keder, W. T. A. Harrison, R. J. Flesher, H. A. Mayer, W. C. Kaska, Inorg. Chem. 1999, 38, 3223-3227.

[157] (a) A. Jansen, H. Görls, S. Pitter, Organometallics 2000, 19, 135-138. (b) P. Deglmann, E. Ember, P. Hofmann, S. Pitter, O. Walter, Chem. Eur. J. 2007, 13, 2864-2879.

[158] G. Albertin, S. Antoniutti, J. Castro, S. García-Fontán, G. Zanardo, Organometallics 2007, 26, 29182930.

[159] M. Lesbre, P. Mazerolles, J. Satgé, The Organic Compounds of Germanium. John Wiley and Sons, London-New York 1971.

[160] R. J. P. Corriu, J. J. E. Moreau, J. Organomet. Chem. 1972, 40, 55-72.

[161] Y. Zhou, D. S. Richeson, J. Am. Chem. Soc. 1996, 118, 10850-10852.

[162] C. Ni, B. D. Ellis, G. J. Long, P. P. Power, Chem. Commun. 2009, 2332-2334.

[163] Y. Xiong, S. Yao, M. Driess, J. Am. Chem. Soc. 2009, 131, 7562-7563.

[164] (a) J. Mautz, G. Huttner, Eur. J. Inorg. Chem. 2008, 1423-1434. (b) M. M. Hossain, H.-M. Lin, S.-G. Shyu, Organometallics 2007, 26, 685-691. (c) Z.-X. Zhang, Y.-R. Li, R.-T. Liu, Z.-X. Chen, L.-H. Weng, X.-G. Zhou, Polyhedron 2007, 26, 4986-4992. (d) A. Bacchi, W. Baratta, F. Calderazzo, F. Marchetti, G. Pelizzi, Inorg. Chem. 2002, 41, 3894-3900. (e) N. Burford, P. Losier, S. Mason, P. K. Bakshi, T. S. Cameron Inorg. Chem. 1994, 33, 5613-5614.

[165] T. Matsumoto, N. Tokitoh, R. Okazaki, J. Am. Chem. Soc. 1999, 121, 8811-8824.

[166] B. Becker, W. Wojnowski, Synth. React. Inorg. Met.-Org. Chem. 1982, 12, 565-582.

[167] M. C. Kuchta, G. Parkin, J. Chem. Soc. Chem. Commun. 1994, 1351-1352. 
[168] Cambridge Structural Database, release 5.29; Cambridge Crystallographic Data Centre: Cambridge, U.K. November 2007.

[169] T. Matsumoto, Y. Nakaya, K. Tatsumi, Organometallics 2006, 25, 4835-4845.

[170] V. Jancik, Y. Peng, H. W. Roesky, J. Li, D. Neculai, A. M. Neculai, R. Herbst-Irmer, J. Am. Chem. Soc. 2003, 125, 1452-1453.

[171] (a) H. C. Allen, L. R. Blaine, E. K. Plyler, J. Chem. Phys. 1956, 24, 35-38. (b) H. C. Allen, E. K. Plyler, J. Chem. Phys. 1956, 25, 1132-1136.

[172] Y. Ding, Q. Ma, I. Usón, H. W. Roesky, J. Am. Chem. Soc. 2002, 124, 8542-8543.

[173] T. Matsumoto, K. Tatsumi, Chem. Lett. 2001, 30, 964-965.

[174] H. Zhu, J. Chai, H. Fan, H. W. Roesky, C. He, V. Jancik, H.-G. Schmidt, M. Noltemeyer, W. A. Merrill, P. P. Power, Angew. Chem. 2005, 117, 5220-5223; Angew. Chem. Int. Ed. 2005, 44, 50905093.

[175] (a) N. Wiberg, J. Organomet. Chem. 1984, 273, 141-147. (b) G. Raabe, J. Michl, Chem. Rev. 1985, 85, 419-509. (c) G. Bertrand, J.-P. Majoral, A. Baceiredo, Acc. Chem. Res. 1986, 19, 17-23. (d) I. C. Tornieporth-Oetting, T. M. Klapötke, Angew. Chem. 1995, 107, 559-568; Angew. Chem. Int. Ed. Engl. 1995, 34, 511-520.

[176] H. R. Kricheldorf, G. Schwarz, J. Kaschig, Angew. Chem. 1977, 89, 570-572; Angew. Chem. Int. Ed. Engl. 1977, 16, 550-552.

[177] (a) W. T. Reichle, Inorg. Chem. 1964, 3, 402-406. (b) J. S. Thayer, R. West, Inorg. Chem. 1964, 3, 406-409. (c) J. S. Thayer, R. West, Inorg. Chem. 1964, 3, 889-893. (d) I. Ruidisch, M. Schmidt, J. Organomet. Chem. 1964, 1, 493-494. (e) S. Cradock, E. A. V. Ebsworth, J. Chem. Soc. A, 1967, 12261229. (f) J. D. Murdoch, D. W. H. Rankin, J. Chem. Soc. Chem. Commun. 1972, 748-750. (g) J. E. Drake, R. T. Hemmings, Can. J. Chem. 1973, 51, 302-311. (h) A. Baceiredo, G. Bertrand, P. Mazerolles, Tetrahedron Lett. 1981, 22, 2553-2556. (i) C. Guimon, G. Pfister-Guillouzo, Organometallics 1987, 6, 1387-1392.

[178] A. C. Filippou, P. Portius, G. Kociok-Köhn, Chem. Commun. 1998, 2327-2328.

[179] M. Veith, A. Rammo, Z. Anorg. Allg. Chem. 2001, 627, 662-668.

[180] M. Denk, R. K. Hayashi, R. West, J. Am. Chem. Soc. 1994, 116, 10813-10814.

[181] N. J. Hardman, C. Cui, H.W. Roesky, W. H. Fink, P. P. Power, Angew. Chem. 2001, 113, 2230-2232; Angew. Chem. Int. Ed. 2001, 40, 2172-2174.

[182] R. J. Wright, A. D. Phillips, T. L. Allen, W. H. Fink, P. P. Power, J. Am. Chem. Soc. 2003, 125, 16941695.

[183] G. N. Lewis, Valence and the Structure of Atoms and Molecules, Chemical Catalogue, New York, 1923.

[184] (a) G. C. Welch, R. R. San Jun, J. D. Masuda, D. W. Stephan, Science 2006, 314, 1124-1126. (b) G. C. Welch, D. W. Stephan, J. Am. Chem. Soc. 2007, 129, 1880-1881. (c) P. A. Chase, D. W. Stephan, Angew. Chem. 2008, 120, 7543-7547; Angew. Chem. Int. Ed. 2008, 47, 7433-7437. (d) P. A. Chase, T. Jurca, D. W. Stephan, Chem. Commun. 2008, 1701-1703.

[185] (a) V. Sumerin, F. Schulz, M. Nieger, M. Leskelä, T. Repo, B. Rieger, Angew. Chem. 2008, 120, 60906092; Angew. Chem. Int. Ed. 2008, 47, 6001-6003. (b) V. Sumerin, F. Schulz, M. Atsumi, C. Wang, M. Nieger, M. Leskelä, T. Repo, P. Pyykkö, B. Rieger, J. Am. Chem. Soc. 2008, 130, 14117-14119.

[186] (a) H. Wang, R. Fröhlich, G. Kehr, G. Erker, Chem. Commun. 2008, 5966-5968. (b) D. P. Huber, G. Kehr, K. Bergander, R. Fröhlich, G. Erker, S. Tanino, Y. Ohki, K. Tatsumi, Organometallics 2008, 27, 5279-5284. (c) K. V. Axenov, G. Kehr, R. Fröhlich, G. Erker, J. Am. Chem. Soc. 2009, 131, 34543455 .

[187] D. Holschumacher, T. Bannenberg, C. G. Hrib, P. G. Jones, M. Tamm, Angew. Chem. 2008, 120, 75387542; Angew. Chem. Int. Ed. 2008, 47, 7428-7432.

[188] (a) W. E. Piers, T. Chivers, Chem. Soc. Rev. 1997, 26, 345-354. (b) W. E. Piers, Adv. Organomet. Chem. 2004, 52, 1-75. (c) A. Y. Timoshkin, G. Frenking, Organometallics 2008, 27, 371-380.

[189] (a) J. D. Masuda, D. M. Walsh, P. Wei, D. W. Stephan, Organometallics 2004, 23, 1819-1824. (b) M. A. Dureen, A. Lough, T. M. Gilbert, D. W. Stephan, Chem. Commun. 2008, 4303-4305. (c) A. D. 
Saverio, F. Focante, I. Camurati, L. Resconi, T. Beringhelli, G. D’Alfonso, D. Donghi, D. Maggioni, P. Mercandelli, A. Sironi, Inorg. Chem. 2005, 44, 5030-5041.

[190] (a) A. S. Goldman, A. H. Roy, Z. Huang, R. Ahuja, W. Schinski, M. Brookhart, Science 2006, 312 , 257-261. (b) U. Fekl, W. Kaminsky, K. I. Goldberg, J. Am. Chem. Soc. 2003, 125, 15286-15287. (c) C. M. Jensen, Chem. Commun. 1999, 2443-2449.

[191] (a) A. J. Arduengo, H. Bock, C. Chen, M. Denk, D. A. Dixon, J. C. Green, W. A. Herrmann, N. L. Jones, M. Wagner, R. West, J. Am. Chem. Soc. 1994, 116, 6641-6649. (b) N. M. Scott, R. Dorta, E. D. Stevens, A. Correa, L. Cavallo, S. P. Nolan, J. Am. Chem. Soc. 2005, 127, 3516-3526.

[192] (a) A. G. Davies, Organotin Chemistry; VCH: New York, 1997. (b) M, Pereyre, J.-P. Quintand, A. Rahm, Tin in Organic Synthesis; Butterworth: Londan, 1987.

[193] (a) W. P. Neumann, Synthesis 1987, 665-683. (b) L. Chen, F. A. Cotton, W. A. Wojtczak, Inorg. Chim. Acta 1996, 252, 239-250.

[194] H. Arakawa, et al. Chem. Rev. 2001, 101, 953-996.

[195] A. R. Katritzky, Comprehensive Organic Functional Transformations Elsevier Pergamon: Amsterdam 1995.

[196] A. He, J. R. Falck, Angew. Chem. 2008, 120, 6688-6691; Angew. Chem. Int. Ed. 2008, 47, 6586-6589.

[197] (a) I. Shibata, T. Suzuki, A. Baba, H. Matsuda, J. Chem. Soc. Chem. Commun. 1988, 882-883. (b) M. H. Fisch, J. J. Dannenberg, M. Pereyre, W. G. Anderson, J. Rens, W. E. L. Grossman, Tetrahedron 1984, 40, 293-298. c) D. L. J. Clive, G. Chittattu, C. K. Wong, J. Chem. Soc. Chem. Commun. 1978, 41-42.

[198] (a) V. Chandrasekhar, S. Nagendran, S. Bansal, M. A. Kozee, D. R. Powell, Angew. Chem. 2000, 112, 1903-1905; Angew. Chem. Int. Ed. 2000, 39, 1833-1835. (b) N. Prokopuk, D. F. Shriver, Inorg. Chem. 1997, 36, 5609-5613. (c) W. Uhl, T. Spies, D. Haase, R. Winter, W. Kaim, Organometallics 2000, 19, 1128-1131.

[199] W. Y. Chan, A. J. Lough, I. Manners, Angew. Chem. 2007, 119, 9227-9230; Angew. Chem. Int. Ed. 2007, 46, 9069-9072.

[200] (a) A. G. Davies, P. J. Smith, Comprehensive Organometallic Chemistry Pergamon: Oxford, U.K. 1982, 2, 584-591. (b) A. G. Davies, Comprehensive Organometallic Chemistry II Pergamon: Oxford, U.K. 1995, 2, 270-277.

[201] J. Schwartz, J. A. Labinger, Angew. Chem. 1976, 88, 402-409; Angew. Chem. Int. Ed. 1976, 15, 333340 .

[202] (a) D. M. Lemal, J. Org. Chem. 2004, 69, 1-11. (b) G. Sanford, Tetrahedron 2003, 59, 437-454. (c) T. Hiyama, Organofluorine Compounds: Chemistry and Applications; Springer: Berlin, 2000.

[203] (a) J. A. Kerr, In CRC Handbook of Chemistry and Physics, 71st ed. R. L. Lide, Ed. CRC Press: Boca Raton, FL, 1990; pp 9.65-9.98. (b) B. E. Smart, Mol. Struct. Energ. 1986, 3, 141-191. (c) B. E. Smart, In The Chemistry of Functional Groups, Supplement D; S. Patai, Z. Rappoport, Eds.; John Wiley \& Sons: New York, 1983; Chapter 14, pp 603-655.

[204] M. Hudlicky, J. Fluorine Chem. 1989, 44, 345-359.

[205] R. E. Florin, W. J. Pummer, L. A. Wall, J. Res. Natl. Bur. Stand. 1959, 62, 119.

[206] L. W. Pineda, V. Jancik, H. W. Roesky, R. Herbst-Irmer, Angew. Chem. 2004, 116, 5650-5652; Angew. Chem. Int. Ed. 2004, 43, 5534-5536.

[207] L. W. Pineda, V. Jancik, R. B. Oswald, H. W. Roesky, Organometallics 2006, 25, 2384-2387.

[208] M. Stender, A. D. Phillips, P. P. Power, Inorg. Chem. 2001, 40, 5314-5315.

[209] A. J. Arduengo III, H. V. R. Dias, R. L. Harlow, M. Kline, J. Am. Chem. Soc. 1992, 114, 5530-5534.

[210] A. J. Arduengo, J. C. Calabrese, F. Davidson, H. V. R. Dias, J. R. Goerlich, R. Krafczyk, W. J. Marshall, M. Tamm, R. Schmutzler, Helv. Chim. Acta 1999, 82, 2348-2364.

[211] W. A. Herrmann, M. Elison, J. Fischer, C. Köcher, G. R. J. Artus, Chem. Eur. J. 1996, 2, 772-780.

[212] G. D. Frey, V. Lavallo, B. Donnadieu. W. W. Schoeller, G. Bertrand, Science 2007, 316, 439-441.

[213] G. H. Spikes, J. C. Fettinger, P. P. Power, J. Am. Chem. Soc. 2005, 127, 12232-12233.

[214] Y. Peng, B. D. Ellis, X. Wang, P. P. Power, J. Am. Chem. Soc. 2008, 130, 12268-12269.

[215] (a) H. Wu, W. Zhou, T. Yildirim, J. Am. Chem. Soc. 2008, 130, 14834-14839. (b) A. Paul, C. B. Musgrave, Angew. Chem. 2007, 119, 8301-8304; Angew. Chem. Int. Ed. 2007, 46, 8153-8156. (c) N. 
Blaquiere, S. Diallo-Garcia, S. I. Gorelsky, D. A. Black, K. Fagnou, J. Am. Chem. Soc. 2008, 130, 14034-14035.

[216] M. K. Denk, J. M. Rodezno, S. Gupta, A. J. Lough, J. Organomet. Chem. 2001, 617-618, 242-253.

[217] L. W. Pineda, V. Jancik, H. W. Roesky, R. Herbst-Irmer, Inorg. Chem. 2005, 44, 3537-3540.

[218] Y. Yang, H. W. Roesky, P. G. Jones, C.-W. So, Z. Zhang, R. Herbst-Irmer, H. Ye, Inorg. Chem. 2007, 46, 10860-10863.

[219] A. L. Casalnuovo, J. C. Calabrese, D. Milstein, Inorg. Chem. 1987, 26, 971-973.

[222] S. Yao, C. van Wüllen, M. Driess, Chem. Commun. 2008, 5393-5395.

[221] H. V. R. Dias, Z. Wang, J. Am. Chem. Soc. 1997, 119, 4650-4655.

[222] M. Driess, S. Yao, M. Brym, C. van Wüllen, Angew. Chem. 2006, 118, 6882-6885; Angew. Chem. Int. Ed. 2006, 45, 6730-6733.

[223] Z. Yang, X. Ma, R. B. Oswald, H. W. Roesky, H. Zhu, C. Schulzke, K. Starke, M. Baldus, H.-G. Schmidt, M. Noltemeyer, Angew. Chem. 2005, 117, 7234-7236; Angew. Chem. Int. Ed. 2005, 44, 70727074 .

[224] (a) B. Gehrhus, M. F. Lappert, J. Organomet. Chem. 2001, 617-618, 209-223. (b) N. J. Hill, R. West, J. Organomet. Chem. 2004, 689, 4165-4183.

[225] H. Ottosson, P. G. Steel, Chem. Eur. J. 2006, 12, 1576-1585.

[226] C.-W. So, H. W. Roesky, R. B. Oswald, A. Pal, P. G. Jones, Dalton Trans. 2007, 5241-5244.

[227] W. Yang, H. Fu, H. Wang, M. Chen, Y. Ding, H. W. Roesky, A. Jana, Inorg. Chem. 2009, 48, 50585060 .

[228] S. P. Kolesnikov, V. I. Shiryaev, O. M. Nefedov, Izv. Akad. Nauk SSSR Ser. Khim. 1966, 584.

[229] S. S. Sen, A. Jana, H. W. Roesky, C. Schulzke, Angew. Chem. 2009, 121, accepted manuscript; Angew. Chem. Int. Ed. 2009, 48, accepted manuscript.

[230] (a) S. Yao, M. Brym, C. van Wüllen, M. Driess, Angew. Chem. 2007, 119, 4237-4248; Angew. Chem. Int. Ed. 2007, 46, 4159-4162. (b) Y. Xiong, S. Yao, M. Brym, M. Driess, Angew. Chem. 2007, 119, 4595-4597; Angew. Chem. Int. Ed. 2007, 46, 4511-4513. (c) Y. Xiong, S. Yao, M. Driess, Organometallics 2009, 28, 1927-1933. (d) S. Yao, C. van Wüllen, X.-Y. Sun, M. Driess, Angew. Chem. 2008, 120, 3294-3297; Angew. Chem. Int. Ed. 2008, 47, 3250-3253. (e) Y. Xiong, S. Yao, M. Driess, Chem. Eur. J. 2009, 15, 5545-5551. (f) A. Meltzer, C. Praesang, C. Milsmann, M. Driess, Angew. Chem. 2009, 121, 3216-3219; Angew. Chem. Int. Ed. 2009, 48, 3170-3173. (g) A. Meltzer, C. Praesang, M. Driess, J. Am. Chem. Soc. 2009, 131, 7232-7233. (h) Y. Xiong, S. Yao, M. Driess, Chem. Eur. J. 2009, 15, 8542-8547.

[231] C. Cui, H. W. Roesky, H.-G. Schmidt, M. Noltmeyer, Angew. Chem. 2000, 112, 4705-4707; Angew. Chem. Int. Ed. 2000, 39, 4531-4533.

[232] (a) E. Fischer, F. Jourdan, Ber. 1883, 16, 2241-2245. (b) E. Fischer, O. Hess, Ber. 1884, 17, 559-568. (c) R. B. van Orden, H. G. Lindwell, Chem. Rev. 1942, 30, 69-96.

[233] (a) R. N. F. Thorneley, R. E. Eady, D. J. Lowe, Nature 1978, 272, 557-558. (b) B. M. Barney, T.-C. Yang, R. Y. Igarashi, P. C. Dos Santos, M. Laryukhin, H.-I. Lee, B. M. Hoffman, D. R. Dean, L. C. Seefeldt, J. Am. Chem. Soc. 2005, 127, 14960-14961.

[234] (a) J. B. Howard, D. C. Ress, Chem. Rev. 1996, 96, 2965-2982. (b) B. K. Burgess, D. J. Lowe, Chem. Rev. 1996, 96, 2983-3011.

[235] (a) J. A. Pool, E. Lobkovsky, P. J. Chirik, Nature 2004, 427, 527-530. (b) P. Avenier, M. Taoufik, A. Lesage, X. Solans-Monfort, A. Baudouin, A. de Mallmann, L. Veyre, J.-M. Basset, O. Eisenstein, L. Emsley, E. A. Uqadrelli, Science 2007, 317, 1056-1060.

[236] W.-C. Chu, C.-C. Wu, H.-F. Hsu, Inorg. Chem. 2006, 45, 3164-3166.

[237] (a) D. J. Alberas, J. Kiss, Z.-M. Liu, J. M. White, Surface Science 1992, 278, 51-61. (b) C. Lim, C. H. Choi, J. Chem. Phys. 2004, 120, 979-987.

[238] (a) N. Wiberg, M. Veith, Chem. Ber. 1971, 104, 3176-3190. (b) W. Clegg, M. Haase, H. Hluchy, U. Klingebiel, G. M. Sheldrick, Chem. Ber. 1983, 116, 290-298. (c) C. Drost, U. Klingebiel, Chem. Ber. 1993, 126, 1413-1416. (d) E. Gellermann, U. Klingebiel, T. Pape, F. D. Antonia, T. R. Schneider, S. Z. Schumatz, Anorg. Allg. Chem. 2001, 627, 2581-2588. 
[239] (a) M. P. Doyle, D. C. Forbes, Chem. Rev. 1998, 98, 911-935. (b) G. Du, B. Andrioletti, E. Rose, L. K. Woo, Organometallics 2002, 21, 4490-4495. (c) W. Kirmse, Angew. Chem. 2003, 115, 1120-1125; Angew. Chem. Int. Ed. 2003, 43, 1088-1093. (d) D. Marcoux, A. B. Charette, Angew. Chem. 2008, 120, 10309-10312; Angew. Chem. Int. Ed. 2008, 47, 10155-10158.

[240] (a) T. M. Trnka, R, H. Grubbs, Acc. Chem. Res. 2001, 34, 18-29. (b) R. R. Schrock, Angew. Chem. 2006, 118, 3832-3844; Angew. Chem. Int. Ed. 2006, 45, 3748-3759. (c) R. H. Grubbs, Angew. Chem. 2006, 118, 3845-3850; Angew. Chem. Int. Ed. 2006, 45, 3760-3765.

[241] H. M. L. Davies, E. G. Antoulinakis, J. Organomet. Chem. 2001, 617-618, 47-55.

[242] D. M. Hodgson, F. Y. T. M. Pierard, P. A. Stupple, Chem. Soc. Rev. 2001, 30, 50-61.

[243] (a) J.-P. Barnier, L, Blanco, J. Organomet. Chem. 1996, 514, 67-71. (b) R. J. Wright, M. Brynda, J. C. Fettinger, A. R. Betzer, P. P. Power, J. Am. Chem. Soc. 2006, 128, 12498-12509.

[244] E. Y. Tsui, P. Müller, J. P. Sadighi, Angew. Chem. 2008, 120, 9069-9072; Angew. Chem. Int. Ed. 2008, 47, 8937-8940.

[245] C. Bibal, S. Mazières, H. Gornitzka, C. Couret, Angew. Chem. 2001, 113, 978-980; Angew. Chem. Int. Ed. 2001, 40, 952-954.

[246] (a) A. W. Johnson, Ylides and Imines of Phosphorus, Wiley, New York, 1993. (b) W. J. Evans, E. Montalvo, T. M. Champagne, J. W. Ziller, A. G. DiPasquale, A. L. Rheingold, J. Am. Chem. Soc. 2008, 130, 16-17.

[247] G. Bertrand, Organosilicon chemistry III: from molecules to materials, Wiley-VCH, 1998, 223-236.

[248] J. Rebek, D. Feitler, J. Am. Chem. Soc. 1973, 95, 4052-4053.

[249] L. A. Carpino, J. Am. Chem. Soc. 1993, 115, 4397-4398.

[250] R. B. Merrifield, J. Am. Chem. Soc. 1963, 85, 2149-2154.

[251] G. Veneziani, S. Shimada, M. Tanaka, Organometallics 1998, 17, 2926-2929.

[252] G. Veneziani, R. Réau. G. Bertrand, Organometallics 1993, 12, 4289-4290.

[253] (a) W. Setaka, K. Hirai, H. Tomioka, K. Sakamoto, M. Kira, J. Am. Chem. Soc. 2004, 126, 2696-2697.

(b) W. Setaka, K. Hirai, H. Tomioka, K. Sakamoto, M. Kira, Chem. Commun. 2008, 6558-6560.

[254] G. Bai, H. W. Roesky, H. Hao, M. Noltemeyer, H.-G. Schmidt, Inorg. Chem. 2001, 40, 2424-2426.

[255] R. Réau. G. Veneziani, G. Bertrand, J. Am. Chem. Soc. 1992, 114, 6059-6063.

[256] S. Fischer, C. Wentrup, J. Chem. Soc., Chem. Comm. 1980, 502-503.

[257] M. Granier, A. Baceiredo, H. Grützmacher, H. Pritzkow, G. Bertrand, Angew. Chem. 1990, 102, 671672; Angew. Chem., Int. Ed. 1990, 29, 659-661.

[258] R. E. Allan, M. A. Beswick, A. J. Edwards, M. A. Paver, M.-A. Rennie, P. R. Raithby, D. S. Wright, J. Chem. Soc. Dalton Trans. 1995, 1991-1994.

[259] A. P. Dove, V. C. Gibson, E. D. Marshall, H. S. Rzepa, A. J. P. White, D. J. Williams, J. Am. Chem. Soc. 2006, 128, 9834-9843.

[260] S. Yao, M. Brym, K. Merz, M. Driess, Organometallics 2008, 27, 3601-3607.

[261] T. A. George, M. F. Lappert, J. Organomet. Chem. 1968, 14, 327-337.

[262] P. Zhao, C. Krug, J. F. Hartwig, J. Am. Chem. Soc. 2005, 127, 12066-12073.

[263] E. Katayev, Y. Li, A. L. Odom, Chem. Commun. 2002, 838-839.

[264] R. A. Howie, W. Moser, Nature 1968, 219, 372-373.

[265] R. S. Tobias, Acta. Chem. Scand. 1958, 12, 198-223.

[266] J. D. Donaldson, S. M. Grimes, S. R. Johnston, I. Abrahams, J. Chem. Soc. Dalton Trans. 1995, 22732276.

[267] W. D. Honnick, J. J. Zuckerman, Inorg. Chem. 1976, 15, 3034-3037.

[268] L. W. Pineda, V. Jancik, J. F. Colunga-Valladares, H. W. Roesky, A. Hofmeister, J. Magull, Organometallics 2006, 25, 2381-2383. 


\section{List of Scientific Contributions}

\section{Publications}

1. Functionalization of Aminophosphanes: Synthesis and X-Ray Crystal Structure of Novel Dilithium and Trilithium Complexes Containing Silicon-Fused Heteronuclear $\mathrm{SiN}_{2} \mathrm{PLi}$ Five-Membered Rings

Anukul Jana, Gerald Schwab, Herbert W. Roesky and Dietmar Stalke

Inorg. Chem. 2008, 47, 8990-8994

2. Facile Access of Stable Divalent Tin Compounds with Terminal Methyl, Amide, Fluoride, and Iodide Substituents

Anukul Jana, Herbert W. Roesky, Carola Schulzke, Alexander Dring, Tobias Beck, Aritra Pal and Regine Herbst-Irmer

Inorg. Chem. 2009, 48, 193-197

3. Cleavage of a N-H Bond of Ammonia at Room Temperature by a Germylene

Anukul Jana, Ina Objartel, Herbert W. Roesky and Dietmar Stalke

Inorg. Chem. 2009, 48, 798-800

4. A Germanium(II) Hydride as an Effective Reagent for Hydrogermylation Reactions

Anukul Jana, Debajyoti Ghoshal, Herbert W. Roesky, Ina Objartel, Gerald Schwab and Dietmar Stalke

J. Am. Chem. Soc. 2009, 131, 1288-1293

5. Reactions of Tin(II) Hydride Species with Unsaturated Molecules

Anukul Jana, Herbert W. Roesky, Carola Schulzke and Alexander Döring

Angew. Chem. Int. Ed. 2009, 48, 1106-1109.

6. Preparation and Structural Characterization of Molecular Al-O-Sn(II) and Al-O-Sn(IV) Compounds Sharanappa Nembenna, Sanjay Singh, Anukul Jana, Herbert W. Roesky, Ying Yang, Hongqi Ye, Holger Ott and Dietmar Stalke

Inorg. Chem. 2009, 48, 2273-2276

7. Oxidative Addition of Ammonia at a Silicon(II) Center and an Unprecedented Hydrogenation Reaction of Compounds with Low-Valent Group 14 Elements Using Ammonia Borane

Anukul Jana, Carola Schulzke and Herbert W. Roesky

J. Am. Chem. Soc. 2009, 131, 4600-4601

8. Unprecedented End-On Nitrogen Insertion of a Diazo Compound into a Germanium(II) Hydrogen Bond and a Comparable Reaction with Diethyl Azodicarboxylate

Anukul Jana, Sakya S. Sen, Herbert W. Roesky, Carola Schulzke, Sudipta Dutta and Swapan K. Pati Angew. Chem. Int. Ed. 2009, 48, 4246-4248.

9. Facile Access of Well-Defined Stable Divalent Lead Compounds with Small Organic Substituents Anukul Jana, Sankaranarayana Pillai Sarish, Herbert W. Roesky, Carola Schulzke, Alexander Dring and Michael John

Organometallics, 2009, 28, 2563-2567

10. A Silylene with a Tricoordinate Silicon Atom as a Ligand for a Metal Complex

Wei Yang, Hao Fu, Haijun Wang, Mingqing Chen, Yuqiang Ding, Herbert W. Roesky and Anukul Jana

Inorg. Chem. 2009, 48, 5058-5060 
11. Stable Compounds of Composition LGe(II)R ( $\left.\mathrm{R}=\mathrm{OH}, \mathrm{PhO}, \mathrm{C}_{6} \mathrm{~F}_{5} \mathrm{O}, \mathrm{PhCO}_{2}\right)$ : Prepared by Addition Reactions

Anukul Jana, Bijan Nekoueishahraki, Herbert W. Roesky and Carola Schulzke

Organometallics 2009, 28, 3763-3766

12. Dehydrogenation of $\mathrm{LGeH}$ by a Lewis Carbene-Borane Pair Under the Formation of L'Ge and its Reactions with $\mathrm{B}\left(\mathrm{C}_{6} \mathrm{~F}_{5}\right)_{3}$ and Trimethylsilyl Diazomethane. An Unprecedented Rearrangement of a Diazocompound to an Isonitrile

Anukul Jana, Ina Objartel, Herbert W. Roesky and Dietmar Stalke

Inorg. Chem. 2009, 48, 7645-7649

13. Hydrostannylation of Ketones and Alkynes with $\mathrm{LSnH}\left[\mathrm{L}=\mathrm{HC}(\mathrm{CMeNAr})_{2} ; \mathrm{Ar}=2,6-i \mathrm{Pr}_{2} \mathrm{C}_{6} \mathrm{H}_{3}\right]$

Anukul Jana, Herbert W. Roesky and Carola Schulzke

Inorg. Chem. 2009, 48, 9543-9548

14. Synthesis and Structural Characterization of Heterobimetallic Bismuth Complexes with Main Group and Transition Metals

Bijan Nekoueishahraki, Anukul Jana, Herbert W. Roesky, Daniel Stern and Dietmar Stalke

Organometallics 2009, 28, 5733-5738

15. A Remarkable Base Stabilized Bis Silylene with a $\mathrm{Si}(\mathrm{I})-\mathrm{Si}(\mathrm{I})$ Bond Sakya S. Sen, Anukul Jana, Herbert W. Roesky and Carola Schulzke Angew. Chem. Int. Ed. 2009, 48, 8536-8538

16. Insertion Reaction of a Silylene into a N-H bond of Hydrazine and a [1+4] Cycloaddition with Diphenyl Hydrazone

Anukul Jana, Herbert W. Roesky, Carola Schulzke and Prinson P. Samuel

Organometallics 2009, 28, 6574-6577

17. Reactivity of Germanium(II) Hydride with Nitrous Oxide, Trimethylsilyl Azide, Ketones, and Alkynes and the Reaction of a Methyl Analogue with Trimethylsilyl Diazomethane

Anukul Jana, Herbert W. Roesky and Carola Schulzke

Dalton Trans. 2010, 39, 132-138

18. Synthesis of Phosphine Substituted $\beta$-Diketiminate Based Isomeric Ge(II) Complexes

N. Dastagiri Reddy, Anukul Jana, Herbert W. Roesky, Prinson P. Samuel and Carola Schulzke

Dalton Trans. 2010, 39, 234-238

19. A Rational Design for an Efficient Synthesis of a Monomeric Tin(II) Hydroxide

Anukul Jana, Sankaranarayana Pillai Sarish, Herbert W. Roesky, Carola Schulzke and Prinson P. Samuel

Chem. Commun. 2010, 46, 707-709

20. An Efficient Route for the Synthesis of a Tin(II) Substituted Carbodiimide from a Diazo Compound Anukul Jana, Herbert W. Roesky, Carola Schulzke and Prinson P. Samuel Inorg. Chem. 2010, 49, 3461-3464

21. Heavier Alkaline Earth Metal Borohydride Complexes Stabilized by $\beta$-Diketiminate Ligand Sankaranarayana Pillai Sarish, Anukul Jana, Herbert W. Roesky, Thomas Schulz, Michael John and Dietmar Stalke

Inorg. Chem. 2010, 49, 3816-3820 
22. Reaction of $\beta$-diketiminate tin(II) dimethylamide $\mathrm{LSnNMe}_{2}\left[\mathrm{~L}=\mathrm{HC}(\mathrm{CMeNAr})_{2} ; \mathrm{Ar}=2,6-i \mathrm{Pr}_{2} \mathrm{C}_{6} \mathrm{H}_{3}\right]$ with ketones and alkynes

Anukul Jana, Ina Objartel, Herbert W. Roesky and Dietmar Stalke

Dalton Trans. 2010, 39, DOI: 10.1039/c002849e

23. Preparation of iron carbonyl complexes of germanium(II) and tin(II) each with a terminal fluorine atom Anukul Jana, Prinson P. Samuel, Herbert W. Roesky and Carola Schulzke J. Fluorine Chem. 2010, doi:10.1016/j.jfluchem.2010.03.011

24. Reaction of Tin(II) Hydride with Compounds Containing Aromatic C-F Bonds Anukul Jana, Herbert W. Roesky, Carola Schulzke and Prinson P. Samuel Organometallics 2010, 29, DOI: 10.1021/om1000106

25. Synthesis and Reaction of Monomeric Germanium(II) and Lead(II) Dimethylamide and the Synthesis of Germanium(II) Hydrazine by Clevage of one N-H bond of Hydrazine

Anukul Jana, Herbert W. Roesky, Carola Schulzke, Prinson P. Samuel and Alexander Döring Inorg. Chem. 2010, accepted manuscript (Manuscript ID: ic-2010-003808)

26. Facile Synthesis of Dichlorosilane by Metathesis Reaction and Dehydrogenation of Dihydrogermane by a Frustrated Lewis Pair

Anukul Jana, Gašper Tavčar, Herbert W. Roesky and Carola Schulzke

Dalton Trans. 2010, submitted manuscript

27. Synthesis and Characterization of $\beta$-Diketiminate Germanium(II) and Tin(II) Bromides Anukul Jana, Gerald Schwab, Herbert W. Roesky and Dietmar Stalke Inorg. Chimica Acta 2010, submitted manuscript

\section{Oral and Poster Presentations at Conferences and International Workshops}

1. Amino-Functionalized Aminophosphine: Precursor for a New Class of Tripodal Ligands, 29 th June 2007, Göttingen, Germany. (Oral presentation)

2. Hydrogermylation of Elemental Sulfur and Carbon Dioxide by using Germylene Hydride LGe(II)H: Formation of a Germanium Dithiocarboxylic Acid Analogue and a Germanium(II) Formate, 4 th July 2008, Göttingen, Germany. (Poster presentation)

3. New Precursor for Oxygen briged Complexes, XXI. Tage der Seltenen Erden-Terrae Rarae 2008, 4-6 th December 2008, Bochum, Germany. (Poster presentation)

4. Germylenes and stannylenes: Congeners of carbenes. Herbert W. Roesky and Anukul Jana, Abstracts of Papers, 237th ACS National Meeting, Salt Lake City, UT, USA, 22-26 th March 2009

5. A Paradigm Change in Hydrogen Transfer Reactions of Compounds with Germanium(II) and Tin(II) Centers, 3 rd July 2009, Göttingen, Germany. (Oral presentation)

6. Preparation of Compounds with Low Valent Elements of Group 14. Herbert W. Roesky, Rajendra S. Ghadwal, Anukul Jana, and Sakya S. Sen, Abstracts of Papers, 19th International Symposium on Fluorine Chemistry, Jackson Hole, Wyoming, USA, 23-28 th August 2009 


\section{Lebenslauf}

Name

Geboren

Staatsangehörigkeit

Schulbesuch

1989-1998

1998-1999

1999-2001

Studium

2001-2004

2004-2006

Promotion

Oktober 2006 -

September 2009
Anukul Jana

27. January 1984 in Krishnakhayratibar

Indisch

Primary School (Class I-IX)

Secondary School Examinations (Class X)

Higher Secondary School Examinations (Class XII)

B. Sc. (Hons.) in Chemie, Calcutta University, Indien

M. Sc. in Chemie, Indian Institute of Technology Kanpur, Indien

Anorganische Chemie Göttingen unter der Leitung

von Prof. Dr. Dr. h.c. mult. H. W. Roesky

Ph.D. Thesis "Unprecedented Synthetic and Reactivity Studies of Compounds with Low Valent Group 14 Elements" 
